Smithsonian

Institution

Libraries

Gift of

DR. FREDERICK

M. BAYER 
G. Dolllun unne:

E. Mareus

Tredenck th. Baye 



\section{MANUEL}

DE

L'HISTOIRE NATURELLE

DES MOLLUSQUES

E $\mathbf{T}$

\section{DE LEURS COQUILLES,}

A Y NT POUR BASE DE CLASSIFICATION CELLE DE M. LE. BABON CUVIER;

PAR M. SANDER RANG,

OFFIGIER AU CORPS ROYAT DE LA MARINE,

Membre correspondant de l'Académie Royale de La Rochelle, des Sociétés Philomatique et d'Histoire Naturelle de Paris, de la Société Linnéenne de Bordeanx, eic.

Ouvrage orné de Planches.

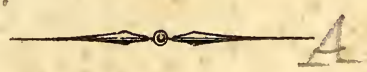

\section{PARIS,}

RORET, LIBRAIRE, RUE HAUTEFEUILLE, aU CoIN DE Gelle du battoik.

MAI I 829 . 
Le même Libraire vient de faire paraítre :

Atras des Mollusques, composé de 5 I planches, représentant la plupart des Mollusques nus et des Coquilles.

Prix, figures noires. ...... $7 \mathrm{fr}$. _- Figures coloriées........ $14 \mathrm{fr}$.

Nota. Il se vend séparément. 


\section{PRÉFACE.}

$e^{2}$

J'AI fait ce livre pour les voyageurs, et particulièrement pour ceux de mes camarades de la marine royale qui, aimant à mettre à profit les loisirs que le service leur laisse dans les pays étrangers, se livrent à l'étude de l'histoire naturelle : j'ose espérer qu'il leur sera utile, et qu'il contribuera à propager parmi eux un goût qui s'étend tous les jours davantage, grâce aux encouragemens que les savans de la capitale donnent à plusieurs d'entre eux, à la manière indulgente avec laquelle ils accueillent jusqu'à leurs moindres travaux, et surtout grâce à l'impulsion qu'ils reçoivent. des hommes éclairés placés en ce moment à la tête de la marine. Désireux de se rendre utiles, et connaissant en outre la protection que $M$. le baron Hyde de Neuville et M. le contre-amiral Halgan accordent à ces sortes de travaux, qu'ils savent eux-mêmes si bien apprécier, plusieurs de ces officiers, marins ou chirurgiens, ont singulièrement contribué, dans ces derniers temps, à augmenter la belle collection du Roi au jardin des Plantes, des productions de toutes les parties du monde, et en même temps à enrichir la science d'une foule de découvertes que, sans leurs secours, on n'eât obtenues quavec le temps, et à grands frais, ou dont peut-être nos voisins nous auraient enlevé la priorité.

Parcourant sans cesse des climats différens, des régions peu fréquentées; vivant au milieu d'une foule d'êtres la plupart mal connus, ou même 
inconnus, qui pourrait, en effet, mieux que les navigateurs, récolter pour la science, observer les mours de ces animaux marins, dont le.plus grand nombre ne peut vivre sous nos climats; étudier leur organisation, tracer leur description détaillée, et en reproduire enfin de parfaites images? C'est en quelque sorte un devoir pour eux; et ne serait-on pas en droit de les accuser d'insouciance si, après s'être récréés ¿d la contemplation de ces objets sans cesse présens à leurs yeux, ils négligeaient de les faire connaître à ceux qui sont chargés d'en faire l'histoire? Et d'ailleurs quelle plus douce occupation peuvent-ils se créer, loin de la patrie; qui saura mieux les distraire de son souvenir; qui occupera mieux leur esprit, au milieu de la solitude de l'Océan et de sa longue monotonie, que l'étude de ces êtres variés, dont les mers sont peuplées avec une si prodigieuse profusion?

Mon but a été de former un genera propre à faciliter l'étude des mollusques, et susceptible peut-être de servir d'introduction aux ouvrages précieux que nous possédons, mais qui, généralement peu à la portée des commençans, et, en outre, d'un prix élevé, ne sauraient toujours leur convenir, surtout à ceux qui désirent prendre une idée de la chose avant que de s'y livrer entièrement. J'ose donc espérer que les savans me sauront gré de mes intentions, et qu'elles pourront, en quelque sorte, faire excuser à leurs yeux l'imperfection de l'ouvrage.

J'ai puisé, comme on doit bien le penser, dans les travaux de ces savans. Il me serait difficile de signaler tout ce qui leur appartient; et d'ailleurs les hommes versés dans l'étude des mollusques le reconnaîtront facilement; je ne ci- 
terai donc que les points principaux. Par exemple, j'ai adopté, pour base de ma classification, les divisions de classes et d'ordres de M. Cuvier, parce que ce sont les plus naturelles, les plus faciles à saisir, les plus accréditées, et les seules qui me semblent devoir subsister, au milieu des diverses classifications imaginées dans ces derniers temps. Je n'y ai apporté de changement qu'en ce qui m'a semblé devoir en éprouver, par suite des progrès de la science : ainsi j'ai réuni les Brachiopodes aux Acéphales testacés, et j'en ai fait le premier ordre de cette classe; dans les Gastéropodes, j'ai introduit l'ordre des $\mathrm{Nu}$ cléobranches, dont le nom est emprunté à M. de Blainville, et que j'ai circonscrit, comme il doit l'être, jusqu'à de nouvelles découvertes, d'après l'étude approfondie que j'en ai faite. Déjà j'avais proposé cette addition à la classe dans une note insérée dans les Mémoires de la Société d'Histoire naturelle, et $M$. de Férussac, bientôt après, l'a adoptée. J'y ai également rapportél'ordre des Cirrhobranches, créé par M. de Blainville pour le genre Dentale, dont la connaissance est due à M. Deshayes.

J'ai emprunté à M. de Lamarck plusieurs compositions de familles, et beaucoup de caractères génériques pour les coquilles; à M. de Férussac, des divisions de second ordre, la composition de quelques familles, et des divisions de genre en sous-genre; ̀̀ M. de Blainville, des familles et la caractéristique de plusieurs genres.

MM. de Férussac et Dorbigny ayant fait tout récemment un travail sur les Céphalopodes, je ne pouvais faire mieux que d'en donner un abrégé pour remplir cette classe.

La partie à laquelle je me suis le plus attaché: 
est celle qui comprend la description des animaux. Elle a été long-temps négligée, et l'on doit surtout à MM. Cuvier et de Blainville les progrès immenses que la science vient de faire sous ce rapport; il reste cependant encore beaucoup à faire; et c'est pourquoi j'appelle particulièrement l'attention des voyageurs sur cet objet. C'est dans les beaux travaux d'Adanson, de Poli et de MM. Cuvier et de Blainville, que j3ai en grande partie puisé les matériaux de ce travail; mais je dois dire qu'à l'exception d'un très petit nombre de genres, j’ai pu depuis quatre années, et pendant le cours de mes voyages, étudier et vérifier sur les animaux vivans les descriptions que, la plupart du temps, ces savans n'avaient pu donner que d'après des animaux morts; aussi m'a-t-il été facile de reconnaître des caractères nouveaux, ou d'en rectifier un certain nombre.

Au moment où se termine l'impression de ce livre, deux circonstances me font regretter de n'avoir pas pú la reculer de quelques mois; d'une part, la publication prochaine de la nouvelle édition du Règne animal de $M$. Cuvier, et de l'autre, l'arrivée de MM. Quoy et Gaimard, dont le voyage si remarquable promet à la science les plus beaux résultats. C'eût été pour moi deux sources dans lesquelles j’eusse puisé de nouveaux et précieux documens pour compléter mon ouvrage. 


\section{DISCOURS SOMMAIRE}

S U R

\section{LES ANIMAUX MOLLUSQUES.}

LA définition adoptée par M. Cuvier pour caractéríser les animaux dont nous allons nous occuper peut se réduire ainsi :

Animaux non complétement symétriques, sans squelette articulé, sans canal vertébral, mais ayant un certain nombre de masses médullaires disposées en différens points du corps, et dont la principale, que l'on peut appeler cerveau, est situcé en travers sur l'oesophage, qu'elle enveloppe d'un collier nerveux.

Presque toujours munis d'un développement de la peau qui recouvre leur corps, et ressemble quelquefois à un manteau, produisant généralement une ou plusicurs lames de substance plus ou moins dure, qui restent cachées dans son épaisseur, ou, ce qui est le plus ordinaire, prennent une grosseur et un développement tels, que l'animal peut se contracter sous leur abri.

La circulation double, c'est-à-dire que la circulation pulmonaire fait toujours un circuit à part et complet; le sang blanc ou bleuatre.

Ils respirent, les uns l'air élastique, et les autres l'eau douce ou saléc.

Cette définition caractérise parfaitement ces animaux; aussi est-elle, à peu de chose près, reproduitè par M. de Lamarck, et sert-elle encore de base à celles de MM. Latreille et de Blainville. Ce dernier 
savant, en lui ajoutant quelques caractères nouveaux, l'a en même temps circonscrite de la manière suivante; et c'est ainsi qu'on peut l'adopter, parce qu'elle est à la fois concise et rigoureusement caractéristique.

Animaux pairs, le corps et ses appendices mous, non articulés, enveloppés d'une peau ou derme musculaire (manteau) de forme variable,-dans ou sur laquelle se développe le plus souvent une partic calcaire (coquille) d'une ou plusieurs (I) pièces.

Circulation complète à sang blanc, à cour essentiellement aortique, et supérieur au canal intestinal, si cie n'est dans les Seiches.

Respiration aquatique ou aérienne; système nerveux composé d'un ganglion cérébriforme, suscesophagien, communiquant avec les ganglions des différentes fonctions, ceux de la locomotion élant latéraux.

Linné et Bruguière désignaient ces animaux sous le nom de vers; Adanson, sous celui de coquillages. M. Cuvier les a réunis sous celui de mollusques, emprunté d'Aristote, mais qui n'était affecté qu'à un certain nombre d'entre eux. Il dérive du mot latin mollis, qui signifie mou; et, en effet, ils sont tous remarquables par la mollesse de leur substance.

IV. de Lamarck a adopté, après M. Cuvier, ceite même dénomination, mais sans l'étendre à tous les animaux que ce savant range dans sa deuxième grande division du règne animal. M. de Blainville a proposé de la remplacer par celle de malacozoaires, et M. Latreille par celle de mantelés. On a encore imaginé quelques noms nouveaux, qu'il est inutile de rappeler; et on en imaginera sans doute bien d'autres encore, qui probablement ne seront pas davantage adoptés. Le seul qui le soit généralement, et que M. de Férussac s'est empressé de répéter dans ses

(I) M. de Blainviile dit : "D'une on deux pièces."

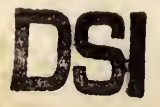


tableaux systématiques, est celui de mollusque, déjà cité, et qui a été proposé par M. Cuvier. Il est sans contredit le plus caractéristique et le plus répandu, et s'il offre quelque inconvénient, ce ne peut être que celui qui résulte d'une certaine discordance entre les différentes acceptions qui lui ont été données par des auteurs justement célèbres.

On s'était tonjours plutôt occupé de la pièce testacée des mollusques que des mollusques eux-mêmes, et l'on donnait à la science qui traite des coquilles le nom de Conchyliologie (1). Mais depuis les recherches de plusieurs naturalistes, et particulièrement depuis la publication des beaux Mémoires anatomiques de M. Cuvier, on a généralement senti que le seul moyen de classer et de caractériser les différens genres d'une manière précise et immuable, était d'étudier et d'indiquer les caractères qui distinguent les animaux entre eux : dès-lors cette branche de l'histoire naturelle s'est présentée sous un point de vue tout différent; et le nom de conchyliologie, quoique devenu bien bon par le fait, se trouvant insuffisant, par suite de sa première application, on a dî en chercher un plus approprié à l'importance que venait d'acquérir cette science. M. de Blainville, celui des zoologistes qui, après M. Cuvier, s'est le plus occupé de l'anatomie des mollusques, a proposé le nom de malacologie, par abréviation de malacozoologie, qui vient des mots grecs $\mu a \lambda a \varkappa o s, \zeta \omega 0 v$ et $\lambda \circ \gamma_{0}$, et signifie discours sur les animaux mous. Il parait avoir reçu la sanction générale.

(I) Ce mot vient du grec conchylion, qui vent dire l'animal d'une coquille; ainsi il serait bien applicable à la science des mollusques telle qu'on la cultive aujourd'bui, et l'on ferait peut-être bien de l'adopter tout-à-fait; cependant, l'habitude l'ayant consacré à la considération seule des coquilles, on ne peut se dissimuler qu'il n'y eût de l'in. cunvénient à une semblable innovation. 
Les mollusques sont d'une substance molle, quelquefois un peu dure et même coriace à l'extérieur, ce qui permet d'en conserver l'enveloppe; quelques uns sont comme gélatineux, et alors susceptibles d'une prompte décomposition après la mort. Leur couleur est généralement pâle, d'un jaune ou blanc sale, et le plus souvent ils sont opaques; cependant un grand nombre présente des couleurs aussi vives que variées, plusieurs sont subtranslucides, et quelques uns d'une transparence qui rivalise avec celle du cristal, mais qui, dans tous les cas, est interrompue dans certains points par l'opacité et la coloration des viscères.

La forme des mollusques varie à l'infini; cependant on peut en distinguer plusieurs principales, dont toutes les autres ne sont, en quelque sorte, que des modifications; et l'on reconnaît facilement, en général, que ces formes principales ou secondaires sont dépendantes du mode de locomotion, ou, en. remontant plus haut, des mours et des lieux d'hahitation : ainsi ceux qui s'écartent des rivages, nagent au milieu des eaux, et s'élèvent vers leur surface au inoyen de nageoires, sont allongés, plus ou moins cylindriques, et propres, par conséquent, à diviser l'élément qu'ils traversent; tels sont les Calmars, les Clios, les Firoles. Les mollusques pélagiens, dont la nature s'est plu à peupler la haute mer avec une prodigieuse profusion, vaguant sans cesse à sa surface, et ne se fixant que très rarement aux corps flottans, présentent une forme aplatie, tiès remarquable chez plusieurs d'entre eux par une dépression horizontale qui, angmentant leur surface, les rend plus propres à flotter sur les eaux sans le secours d'organes locomoteurs. Tels sont ces innombrables Glaucus dont les mers équatoriales sont remplies, les Briarés, les Ptérosomes, etc. Les mollusques littoraux, que l'on nomme ainsi parce qu'ils ne s'éloignent jamais des rivages où ils sunt nés, et 
ce sont les plus nombreux, présentent d'autres combinaisons dans leur forme, selon qu'ils sont libres ot: fixés, et selon la nature des lieux qu'ils habitent; ils n'ont point de nageoires, ou seulement, ce qui est très rare, quelques faibles expansions latérales, propres à les soutenir quelque temps, mais incapables de les porter au loin des rivages. Les uns vivent libres sur les rochers, et s'y trainent lentement, à l'aide d'une surface plane qui leur sert à ramper. Ceux-ci sont plus ou moins allongés et symétriques, plats en dessous, plus larges et bombés vers le milieu, se rétrécissant aux extrémités; et le plus grand nombre d'entre eux, qui est muni d'une coquille, tient renfermé dans cette pièce accessoire un développement de la partie dorsale, contourné en spirale, et que l'on nomme tortillon, qui contient sous cet abri divers organes des plus propres à la vie. De ce nombre sont les Aplysies, les Limaçons, les Buccins, etc. Les autres, qui ne se meuvent qu'avec peine, et uniquement pour s'enfoncer dans le sable ou s'abriter sous une légère couche de vase, ou qui ne se meuvent jamais, sont, en général, assez. allongés, plus ou moins comprimés, et très variables dans la proportion de leur diamètre. Tels sont les Peignes, les Solens, les Huîtres, etc.

Du reste, il n'est pas possible d'assigner des formes constantes pour chaque classe de mollusques; et il est facile de s'assurer que l'on peut passer de l'une de ces formes à une autre par des combinaisons intermédiaires aux deux : ainsi les Nucléobranches forment évidemment un exemple de ce passage, si on les. considère entre les Ptéropodes et les Gastéropodes.

On nomme manteau, chez les mollusques, une enveloppe musculaire très contractile, souvent rude et coriace, qui les entoure, et se montre parfois d'une ampleur remarquable. Lorsque ces animaux sont inquiétés, ils se contractent dans leur nanteau, qui est le seul abri de quelques uns. Ce que l'on nomme géuéralement coquille ou test, et qui se rencontre dans 
le plus grand nombre d'entre eux, en est le produit; et c'est le véritable moyen que la nature a adopté pour les défendre, soit lorsque cette coquille est extérieure, et capable de donner refuge à l'animal tout entier, soit quand elle est interne et rudimentaire, propre seulement à préserver d'un choc étranger les principaux viscères, qu'elle recouvre toujours.

Le manteau n'est pas également dur et épais chez tous les mollusques; il l'est peu chez ceux qui sont pourvus d'une coquille; mais il l'est à un très haut degré chez ceux qui, habitant sur les rivages, parmi les rochers, en sont privés. Les mollusques pélagiens, qui ne sont point exposés comme ceux-ci à être sans cesse heurtés par les corps qui les environnent, l'ont constamment très mince, soit qu'ils aient une coquille, soit qu'ils se montrent nus.

C'est du manteau que dépendent chez les mollus. ques les organes locomoteurs; c'est en effet à lui qu'appartient cette surface plane que l'on nomme pied chez les Gastéropodes, et au moyen de laquelle ils rampent sur les rochers; ces lobes latéraux qui, dans quelques uns, viennent se croiser sur le dos de la coquille, et servent parfois à la natation; ces nageoires égales et symétriques, à l'aide desquelles d'innombrables Ptéropodes s'agitent saus cesse au milieu des mers, et ces bras armés de ventouses qui couronnent la tête des Céphalopodes.

Le manteau varie considérablement de forme; tantôt c'est un large bouclier recouvrant la surface dorsale du mollusque, tantôt ce sorit deux lobes se réunissant en dessus pour laisser entre eux un vaste canal où circule l'élément ambiant; une autre fois, c'est simplement un sac régulier ou irrégulier, présentant quelques orifices pour les différentes fonctions. Enfin, chez quelques mollusques, ses bords sont simples et unis, tandis que chez d'autres, ils présentent des franges plus ou moins agréablement composées, ou de longs filamens tentaculaires. 
Le manteau offre encore un bel ornement dans la couleur dont il est revêtu pendant l'état de vie; et les znollusques pélagiens surtout sont richement partagés sous ce rapport. Enfin, il sécrète un mucus qui devient toujours très abondant quand on irrite l'animal, et qui répand quelquefois une odeur fort désagréable.

La coquille est, comme nous l'avons déjà dit, produite par le manteau : elle se compose, dans des proportions différentes, d'une partie animale et d'une partie calcaire, qui se durcissent bientòt au contact de l'air ou de l'eau, et acquièrent cette consistance que l'on remarque dans le plus grand nombre. Pour la former, le mollusque dépose successivement des couches les unes en dedans des autres, de manière que les plus nouvelles, qui sont en dedans, dépassent les plus anciennes, qui sont par conséquent les plus petites; et c'est par ce moyen que la coquille augmente insensiblement de volume. Différentes combinaisons dans l'application de ces couches la rendent feuilletée, compacte ou fibreuse, et déterminent souvent à sa surface des stries plus ou moins régulières.

La plupart des coquilles sont dures, épaisses, crétacées et opaques, se brisant avec facilité; c'est que chez elles la partie calcaire domine sur la partie animale : mais d'autres présentent une structure cornée et subtranslucide, plus ou moins mince, fragile, vitrée, quelquefois, maies c'est assez rare, flexible et membraneuse; c'est que, dans celle-ci, la partie animale domine, au contraire, sur la partie calcaire, qui, dans plusieurs cas même, disparait entièrement. Le plus grand nombre de ces dernières sont pélagiennes; et l'on conçoit alors qu'il n'était pas nécessaire qu'elles fussent bien solides, puisque les animaux qui les forment habitent les hautes mers, où ils peuvent impunément s'agiter sans craindre d'être. heurtés.

Les coquilles dont nous venons de parler sont ex-. 
térieures et enveloppantes; mais celles que l'on trouve quelquefois dans l'épaisseur du manteau sont plus ou moins cornées et flexibles, ou plutôt membraneuses; elles sont souvent un peu transparentes, comme dans les Aplysies, Pleurobranches, etc., ou même vitrées, comme dans les Sigarets, Calmars, etc., et plusieurs d'entre elles acquièrent avec l'âge une conche calcaire qui revêt leur face inférieure, et sert à les solidifier. Du reste, ces coquilles doivent être considérées comme étant, jusqu'à un certain point, rudimentaires.

C'est surtout par la forme que les coquilles varient; aussi est-ce par là que l'on a presque toujours cherché à établir les caractères qui distinguent les familles et les genres. Nous ne ferons pas l'énumération des formes principales que l'on remarque; nous nous contenterons d'en signaler quelques unes. Les coquilles extérieures présentent une ou plusieurs pièces, que l'on désigne ordinairement sous le nom de valves; ainsi celles qui n'ont qu'une seule pièce sont univalves; celles qui en ont deux, bivalves, et celles qui en ont un plus grand nombre, plurivalves ou multivalves. M. de Blainville donne encore le nom de subbivalves à celles dans lesquelles, outre une pièce analogue à celle qui constitue les coquilles univalves, il y en a encore une seconde, qui sest à fermer plus ou moins complétement l'ouverture, et que l'on nomme opercule, et celui de tubivalves à celles qui, munies comme les bivalves de deux pièces principales, en présentent une troisième en forme dé tube, enveloppant les deux autres. Quant anx coquilles intérieures, elles ne sont jamais que d'une seule pièce.

Les coquilles extérieures univalves présentent généralement deux formes principales, tellement modifiées ensuite, que l'on peut passer de l'unc à l'autre par une transition presque insensible. Dans l'une de ces formes, elles présentent un cône plus ou moins élevé ou aplati, droit ou oblique, régnlier ou ircé- 
gulier, à sommet obtus, recourbé ou même spiral. Cette forme est certainement la plus simple. Dans l'autre, c'est un cône allongé, entièrement contourné en spirale régulière, de manière à former par l'ensemble de ses tours un disque arrondi, ou un cylindre, ou, ce qui est le plus ordinaire, un nouveau cône toujours droit, et plus ou moins court ou élevé. L'animal de quelques unes de ces coquilles forme, lorsqu'il se contracte pour y rester long-temps renfermé, une sorte de cloison mince, plus ou moins calcaire, qui en ferme complétement l'entrée, et que l'on désigne par le nom d'épiphragme. Cette pièce accessoire, et tont-à-fait temporaire, est détruite sitôt que l'animal la brise nour s'étendre hors de sa coquille. Pour la plupart des autres, c'est une pièce constamment dépendan'e de l'animal, auquel elle adhère par une partie de sa surface; l'opercule enfin. Il est complet, c'est-à-dire de même forme et de même grandeur que l'ouverture qu'il doit fermer; ou incomplet, c'est-à-dire plus petit, et plus ou moins dissemblable à la forme de l'ouverture. Dans ce dernier cas, il est quelquefois rudimentaire. Sa nature est calcaire ou cornée. Dans le premier cas, c'est une pièce épaisse et complète; dans le second, elle est mince, plus ou moins opaque, et complète ou non. Sa surface présente presque toujours des stries qui indiquent dans sa formation un mouvement spiral très prononcé. La considération de cette pièce, qui n'a encore bien été étudiée que par M. de Blainville, est d'une grande importance, et peut servir avantageusement pour caractériser les genres.

Les coquilles bivalves sont soumises à un mode de construction tout différent, quoiqu'un grand nombre d'elles présentent encore d'une manière quelquefois assez distincte une disposition spirale dans quelques unes de leurs parties. Elles varient beaucoup dans la forme de leurs deux valves : cependant celles-ci son\& 
toujours concaves en dedans, et s'appliquent l'une contre l'autre de manière à laisser entre elles un espace plus ou moins considérable pour loger l'animal. Il $\mathrm{y}$ a quelques cas, peu nombreux, où ces deux valves sont accompagnées d'une ou plusieurs pièces accessoires, ou bien d'un tube destiné à rendre plus complète encore l'enveloppe testacée qui protége le mollusque.

Dans les coquilles multivalves, les différentes pièces sont soudées ou comme articulées entre elles, ou bien elles sont simplement rapprochées, et maintenues dans une position constante par le manteau de l'animal. Le nombre de ces pièces varie selon les genres, et quelquefois devient indéterminé; mais il y en a toujours de principales, qui sont invariables dans leur nombre.

Les coquilles extérieures sont, en général, ornées de côtes, ou de stries, ou de différentes sortes d'armures en épines, en crêtes ou en productions rameuses. Plusieurs d'entre elles sont tapissées d'une belle nacre à l'intérieur; d'autres sont revêtues à l'extérieur d'un émail qui les rend extrêmement polies et luisantes, et le plus grand nombre est surtout remarquable par la diversité et l'éclat des couleurs, ou par leurs combinaisons, qui présentent à l'œil les dessins les plus variés et les plus bizarres. On retrouve toutes les couleurs parmi les coquilles; cependant celles qui se manifestent le plus rarement sont le bleu et le vert, encore n'est-ce généralement qu'à l'intérieur des valves. On ne connaît pas bien encore le phénomène de la coloration des coquilles; cependant on a déjà réuni plusieurs observations qui apprennent que la lumière ne lui est point étrangère.

La beauté d'un grand nombre de ces productions testacées les a toujours fait rechercher avec avidité par les nombreux amateurs de Conchyliologie; aussi ont-elles donné lieu entre eux à une rivalité qui, stimulant la cupidité de quelques voyageurs, a rendu 
à la science le service de multiplier sous ses yeux les sujets d'observation. Malheureusement, ce goût ne s'est d'abord manifesté que pour celles qui sont remarquables par leur éclat ou par les particularités de leurs formes; et on a long-temps négligé celles que la nature n'a pas si généreusement dotées; aussi sontelles beaucoup moins connues encore aujourd'hui, que les voyageurs les recherchent avec non moins d'avidité, persuadés que c'est parmi elles seulement que l'on peut trouver avec abondance des objets nouveaux et des observations intéressantes. Ises coquilles intérieures sont dans le même cas; elles ont toujours été négligées, soit que l'on n'ait point soupçonné l'existence d'un grand nombre d'entre elles, soit qu'elles ne présentassent aucun attrait à ceux qui forment des collections de luxe. Ces coquilles sont généralement aplaties, faiblement concaves, ou seulement courbes; et quelques unes, moins imparfaites, présentent un sommet qui indique déjà le mouvement spiral, qui se développera plus tard dans d'autres coquilles. Elles sont blanches dans leurs parties calcaires, et toujours plus ou moins rembrunies dans leurs parties membraneuses.

Il est encore à propos de parler, au sujet des coquilles univalves et bivalves, de l'épiderme qui fort souvent recouvre leur surface. Cette production est animale, plus ou moins épaisse, se desséchant sur le test qu'elle enveloppe, quelquefois lisse, et, dans des cas fort rares, pilifére. G'est le dernier vestige de cette partie de la peau qui, chez quelques mollusques nus, recouvre la coquille.

Les coquilles enveloppantes ne sont jamais indépendantes des mollusques qui les produisent, ou du moins on n'en connaît qu'un seul exemple (1). Elles adhèrent au corps de l'animal par le moyen de mus-

(r) L'Argouaute. 
cles particuliers; mais il n'en est pas de même des coquilles intérieures : renfermées dans le manteau, elles n'ont pas besoin d'être retenues par des muscles, et les replis de cette enveloppe suffisent pour la maintenir.

L'adhérence de l'animal à la coquille se fait, sur quelques points de la face interne de celle-ci, au moyen d'un ou de plusieurs faisceaux musculaires. Pendant la vie, cette adhérence est très forte; mais elle s'affaiblit après la inort, et la coquille en conserve presque toujours des empreintes. On nomme celles-ci impressions musculaires, et leur étude est de la plus grande importance.

Dans les mollusques qui sont pourvus de coquilles en forme de gaîne ouverte à un bout, et fermée à l'autre, comme plusieurs Ptéropodes, par exemple, rette adhérence se fait au fond de la coquille, par l'extrémité postérieure d'un grand muscle longitudinal et dorsal, que l'on nomme muscle rétracteur, et l'impression musculaire est rarement visible. Dans ceux à coquilles recouvrantes, comme les Patelles, elle se fait, vers le milieu de leur profondeur, par un muscle disposé plus ou moins complétement en fer à cheval, et généralement l'impression musculaire, qui conserve cette même forme, est très distincte. Dans ceux à coquilles spirales, telles que les Toupies, Buccins, etc., elle se fait à peu près comme dans le premier cas; mais ici c'est autour de la columelle, sur laquelle elle laisse quelquefois des traces évidentes.

Le mode d'adhérence est plus compliqué pour les mollusques qui se revêtent d'une coquille bivalve; et c'est alors surtout que l'nn tire des impressions musculaires des caractères importans pour la distinction des genres et leur classification.

Dans les uns, un seul muscle lie l'animal à ses deux valves, en s'y fixant par les deux extrémités. Il est alors central ou subcentral. Dans les autres, il y en 
a deux, disposés de la même façon, mais l'un en avant, et l'autre en arrière. Ces muscles prennent le nom de muscles adducteurs; et leurs impressions, qui sont presque toujours visibles, ceux d'antérieur pour celle qui provient du muscle antérieur, et de postérieur pour celle qui lui est opposée.

Il arrive souvent, comme dans les Moules, et particulièrement les Pholades; qu'une seule de ces impressions est bien visible; mais on découvre la seconde en la cherchant avec attention, et l'on y est conduit par ce que l'on nonme l'impression paléale. Celle-ci se remarque dans le plus grand nombre des mollusques à deux muscles adducteurs; elle résulte de l'adhérence des bords du manteau avec les deux valves; elle est toujours assez distincte, plus ou moins parallèle au bord de la coquille, et s'étend de l'impression musculaire antérieure à la postérieure, en formant souvent près de celle-ci une profonde excavation, qui est l'indice certain de l'existence d'un organe tubuleux situé à l'extrémité postérieure de l'animal.

Dans les coquilles multivalves, c'est encore par le moyen de faisceaux de fibres musculaires, et par les bords du manteau, que l'animal s'attache à sa pièce testacée.

Après avoir examiné l'enveloppe des mollusques, nous allons, pour compléter cet aperçu de leur organisation extérieure, parler de la tête, des organes locomoteurs, des orifices de la génération et du canal intestinal, áinsi que de l'appareil de la respiration.

La tête des mollusques n'est pas toujours distincte; elle l'est dans les Céphalopodes, quelques Ptéropodes et tous les Gastéropodes; mais elle ne l'est pas dans les autres Ptéropodes, non plus que dans ces mollusques si nombreux dont la coquille est toujours pourvue de deux ou plusieurs pièces, à l'exception nourtant de l'Oscabrion, qui est un Gastéropode. Parmi les mollusques à tête distincte, on en re- 
marque chez qui cette partie est séparée du corps par un étranglement en forme de cou; tels sont les Seiches, les Calmars, les Clios, etc. D'autres n'ont point cet étranglement, comme les Limaces, etc.; et il en est enfin chez qui les contours de la tête sont encore moins faciles à saisir, soit parce qu'elle est très déprimée, comme dans les Doris, soit parce qu'elle se prolonge en avant en une sorte de trompe ou mufle proboscidiforme, presque aussi gros qu'elle, comme on le voit dans les Carinaires et les Cyclostomes. Les mollusques chez qui la tête n'est point distincte sont ceux qui, au lieu d'avoir cette partie saillante et avancée, l'ont, au contraire, rentrée et plus ou moins cachée. La bouche qui la termine toujours indique, dans tous les cas, sa position. Les Cléodores, les Cuviéries et quelques autres genres encore, en offrent des exemples parmi les Ptéropodes. Il en est de même de tous les Lamellibranches et des Cirripèdes.

Les parties de la tête que nous avons à étudier sont la bouche, les tentacules, les yeux, et des appendices servant à la préhension et à la locomotion.

La bouche termine toujours antérieurement la tête du mollusque; elle est souvent en dessous, mais jamais en dessus, et c'est pour cela que les mollusques pélagiens, tels que les Carinaires, les Janthines et les Glauques, sont obligés de se tenir continuellement dans une position renversée, au-dessous de la surface de la mer. La bouche est fendue en long, comme dans les Aplysies, ou presque triangulaire, comme dans certains Ptéropodes et les Carinaires; quelquefois, comme dans les Thétys, elle s'ouvre à l'extrémité d'une petite trompe, et le plus souvent simplement à fleur de tête. Elle est dans un grand nombre de cas accompagnée d'appendices labiaux au nombre de quatre, ce sont les Lamellibranches; d'autres fois ses bords forment une sorte de bourrelet comme dans plusieurs Gastéropodes nus. 
Enfin, il arrive aussi qu'elle est munie d'appendices latéraux en forme de tentacule, comme dans les Aplysies, ou bien une sorte de voile l'enveloppant largement et circulairement, comme dans les Thétys, les Mélibées, etc., ou la recouvrant en dessus seulement, comme dans les Tritonies, les Cérites, etc.

Les tentacules varient en nombre, mais ils sont toujours pairs. Chez certains mollusques, ils ne sont point apparens, tels sont les Acères; d'autres n'en ont qu'une seule paire, comme les Planorbes, etc., ou deux, comme les Limaçons, ou trois, comme quelques Éolides. Il en est même qui ont la tête surmontée encore d'un plus grand nombre de tentacules, ou plutôt alors d'expansions tentaculaires, tels sont les Polycères et les Dentales. Ces organes varient surtout par la forme; ceux qui dépendent des lèvres, et que I'on nomme pour cela tentacules labiaux, sont généralement élargis et plus courts. Les autres, auxquels on donne le nom de tentacules postérieurs, sont ou coniques, ou cylindriques, et plus ou moins allongés. Plusieurs sont fendus longitudinalement à leur sommet, comme dans les Aplysies, ou bien en forme de massue, soit qu'ils composent une seconde paire, soit qu'ils soient seuls, comme dans les Doris, ou sétacés, comme dans les Éolides et les Carinaires, ou terminés par un renflement, comme dans les Cyclostomes, ou aplatis et triangulaires comme dans les Limnés. Enfin, il en est encore qui se montrent plus compliqués en se terminant par une sorte de petite capsule, du milieu de laquelle s'élève un appendice conique : tels sont les Scyllées, les Mélibées, etc.

Les tentacules sont les principaux organes du tact, chez les mollusques; ils paraissent aussi quelquefois devoir aider à la natation : tels sont ceux des Limnés, des Philliroés, et les tentacules labiaux de certaines espèces d'Aplysies. Ils sont en général plus ou moins complétement rétractiles, les uns, comme dans les 
Limaces, en rentrant en eux-mêmes la pointe la première; les autres, comme dans les Tritonies, en rentrant dans un étui qui entoure leur base, ou simplement dans une cavité comme chez les Clios; pour un grand nombre, ce n'est qu'une contraction incomplète.

Les yeux des mollusques sont constamment au nombre de deux ; mais tous ces animaux n'en sont pas également pourvus. On en trouve chez la plus grande partie des céphalés, mais jamais chez les acéphalés. Les mollusques pélagiens errans en sont généralement privés; et à quoi servirait en effet, au plus grand nombre d'entre eux, la présence de ces organes? puisque étant obligés, sans exception, de se tenir dans une position renversée, ils ne pourraient s'en servir à la surface de l'eau, où ils viennent chercher leur nourriture. Cependant les Atlantes et les Carinaires en sont pourvus; mais il est à remarquer que, dans ces animaux, la partie antérieure qui se recourbe en dessus et en arrière (c'est-à-dire vers la partie ventrale), comme le cou d'un cygne, permet aux yeux de se diriger eil haut. MM. Quoy et Gaymard ont bien indiqué deux points noirs sur la tête des Briarée, comme pouvant être les yeux, mais ils ne sont pas sûrs que ce soit ces organes, et il y a tout lieu d'en douter, s'il est permis d'en juger par analogie avec les Glauques, qui forment un genre extrêmement voisin.

Les yeux des mollusques sont quelquefois très grands, et situés sur les côtés de la tête, comme dans les Céphalopodes; mais dans tous les autres mollusques qui en ont, ils s'y montrent très petits, quelquefois à peine distincts, et différemment situés au-dessus de la tête. Ils sont sessiles ou pédiculés, ou placés sur les tentacules. C'est surtout dans le premier cas qu'ils sont peu apparens; alors, ils sont situés de chaque côté de la partie postérieure de la tête, en avant des 
tentacules postérieurs, comme dans les Aplysies, ou en arrière, comme dans les Carichies, ou en dedans, comme dans les Auricules, ou en dehors, comme dans les Mitres. Lorsqu'ils sont pédiculés, ils sont toujours un peu plus distincts, et situés en dehors des tentacules; quelquefois le pédicule n'est qu'une petite éminence, comme dans les Nérites, etc. D'autres fois il est plus allongé, moins que les tentacules, comme dansles Ampullaires, ou plus que les tentacules, comme dans les Strombes. Enfin, lorsque les yeux sont situés sur les tentacules mêmes, ils sont encore î̀̀s distincts, et on les trouve ou au sommet, comme dans les $\mathrm{Li}$ maçons, ou près du sommet, comme dans les Cônes, ou plus près de la base que du sommet, comme dans les Ovules; mais, dans tous les cas, à la partie extérieure.

La tête présente encore d'autres organes; ceux-ci servent à la fois à la préhension et à la locomotion; on ne les rencontre que dans les Céphalopodes, au nombre de huit, de dix, on davantage, disposés autour de la bouche, et on les désigne sous le nom de bras. Ces organes sont plus ou moins courts ou allongés; dans le premier cas, ils sont en forme de pyramide, et accompagnés en outre de deux autres bras beaucoup plus allongés et terminés en massue, comme on le voit dans les Seiches, les Calmars. Dans le second cas, ils sont tous semblables, comme dans les Poulpes, ou bien deux d'entre eux se dilatent de manière à former deux sortes de voiles membraneuses, telles qu'on les voit dans les Argonautes. Tous ces organes sont munis sur leur longueur, ou sur une partie de leur longueur, d'une ou plusieurs rangées de ventouses armées de denticules, ou même quelquefois de crochets, au moyen desquelles ces animaux saisissent les corps avec une grande force. Nous allons parler de nouveau des bras des Céphalopodes, en traitant des organes locomoteurs.

C'est surtout dans la diversité qui règne parmi ces 
organes (r), qu'il y a lieu d'admirer la sage prévoyance avec laquelle la nature a présidé à l'organisation des mollusques, soit pour leur donner les moyens de se traîner sur les rivages, soit pour les conduire au milieu de l'immensité des mers, et les diriger à leur surface; aussi les naturalistes qui se sont le plus occupés de ces animaux ont-ils toujours été frappés à la vue des divers moyens qu'elle a employés pour y parvenir; et M. Cuvier, dont le génie a si bien saisi l'ensemble de ces différentes surtes d'organisations, si dépendantes des mcurs, a-t-il établi en partie, sur leurs considérations, les coupes les plus élevées de sa distribution méthodique. Ce qui fait bien connaître l'excellence de cette distribution, c'est qu'elle n'a point paru un seul instant insuffisante aux progrès de la science et aux nombreuses découvertes qui ont été faites dans ces derniers temps, et qu'elle n'a pu être avantageusement remplacée par celles qui ont été imaginées depuis. Il est même à remarquer que lorsque des naturalistes ont cherché à en créer de nouvelles, ils n'ont fait à peu près que changer la nomenclature; ou bien ils ont adopté une nouvelle série de caractères pour désigner les mêmes classes et les mémes ordres, ou, enfin, changé entre elles les différentes acceptions des mots de classe, d'ordre, etc., de sorte que les mêmes animaux se sont encore, à peu de chose près, groupés comme auparavant. (2)

(1) Les bornes étroites que nous sommes obligés de mettre à ce Discours ne nous permettent pas de traiter de la locomotion autrement que sous le rapport de la translation.

(2) En effet, les classes des Céphalophores, des Nématopodes, etc., et l'ordre des Hétérobranches de $\mathbf{M}$. de Blainville, sont absolument les mêmes que les classes des Céphalopodes, des Cirrhopodes, etc., et l'ordre des Acéphales sans coquilles de M. Cuvier. D'uy autre côté, les. 
On peut considérer les organes locomoteurs des mollusques comme applicables à deux usages différens, la reptation et la natation. Nous pourrions les étudier sous ces deux points de vue, mais nous aimons mieux, pour plus de clarté, les suivre tour à tour dans les différentes classes qu'ils ont contribué à établir.

Dans chacune de ces classes, il y a un organe locomoteur principal; quelquefois il y en a de secondaires, et souvent l'organe locomoteur principal éprouve différentes sortes de modifications. Nous allons exaıniner chaque classe dans ces trois points.

L'organe locomoteur des Céphalopodes se compose des bras dont nous avons déjà parlé, et qui couronnent la tête, au nombre de huit, dix, ou davantage; c'est par le moyen de ces bras et des ventouses, dont ils sont garnis dans leur longueur, que l'animal, dans quelque position que ce soit, et souvent même la tête en bas, se traîne de rocher en rocher, et parcourt lentement, il est vrai, des espaces assez grands. Ces bras sont doués d'une grande force musculaire, et leurs ventouses s'attachent tellement aux corps, qu'on déchirerait plutôt l'animal que de lui faire lâcher prise.

Lorsque les bras sont grands, comme dans les Poulpes, ils suffisent pour soutenir le imollusque sur les eaux, et le conduire à une très grande distance des rivages; mais lorsqu'ils sont courts comme dans les Céphalopodes microscopiques, ou comme dans les Seiches et les Calmars, ils ne sont que d'une faible ressource pour la natation; aussi, les Céphalopodes microscopiques paraissent être des mollusques littoraux, et les autres sont-ils munis d'organes loco-

ordres des Lamellibranches et des Faliobranches du premier sont les mêmes que les classes des Acéphales testacés et des Brachiopodes du second. 
moteurs secondaires qui suppléent à l'insuffisance des bras pour la natation. Ces nouveaux organes sont des nembranes natatoires dont le manteau est pourvu, et qui varient dans leur forme et leur disposition parmi les différens genres. Chez les Sépioteuthes et les Seiches, par exemple, elles sont étroites et longitudinales, placées une de chaque côté de l'animal, et leur puissance ajoute considérablement à celle des bras. Chez les Onychoteuthes et les Calmars, elles sont plus courtes, mais plus larges, et de forme triangulaire, implantées assez près l'une de l'autre à la partie dorsale et postérieure du mollusque, de manière à former, par leur réunion, un rhombe. Chez les Cranchies, ce sont deux petites membranes arrondies et très sap. prochées, situées tout-à-fait à la partie postérieure du manteau.

Quant aux modifications que l'on pent remarquer dans l'organe locomoteur principal des Céphalopodes, elles sont peu nombreuses; cependant on disingue particulièrement celles-ci. Quelquefois, comme dans les Argonautes, deux des huit bras s'élargissent en éventail, de manière à présenter chacun une vaste. surface; il paraît que ces animaux se servent de ces deux organes comme de deux voiles, qu'ils exposent au vent pour en recevoir le mouvement; cependant nous n'avons jamais pu vérifier ce fait, quoique nous. ayons fréquemment rencontré des Argonautes à la surface de la mer. D'autres fois, comme dans les Poulpes. et les Elédons, les bras sont réunis à leur base par des membranes qui, certainement, doivent contribuer à faciliter la natation.

D’après cet examen, on voit que les mollusques: Céphalopodes doivent varier considérablement dans. leurs moeurs, puisqu'ils peuvent être ou litıoraux, ox pélagiens, ou l'un et l'autre à la fois. J'ajouterai ì cela que $\mathbf{M}$. Dorbigny a reconnu que plusieurs d'entre, eux demeurent fixés aux corps par quelques parties: de leur enveloppe ou de leur coquille; ceux-ci sonte 
sans doute privés d'organes locomoteurs, et les bras ne sont plus pour eux que des organes de préhension; ce cas ne se trouve que parmi les Céphalopodes microscopiques.

L'organe locomoteur des Ptéropodes est tout différent, ou du moins, celui qui n'était que secondaire chez les Céphalopodes, devient le principal dans ces premiers. Appelés à habiter les hautes mers, et ne devant se fixer aux corps flottans que rarement et momentanément, un organe pour la reptation leur devenait inutile, mais il était nécessaire qu'ils en eussent pour la natation. Ils ont en effet deux nageoires membraneuses, grandes, fortes, égales, placées en avant et sur les côtés, et par l'agitation desquelles ils vaguent presque sans cesse á la surface ou près de la surface de la mer. Au moyen de cette organisation, leurs mouvemens sont généralement très vifs; c'est un sautillement précipité et continuel que rien ne maitrise, si ce n'est quelquefois, mais incomplétement, de petites expansions membraneuses dépendantes du manteau, et qui s'étendent plus ou moins à la partie postérieure du corps, comme chez les Hyales. Les Ptéropodes ne se fixent que rarement; cependant nous avons assez souvent rencontré les Créseis, rassemblées en nombre considérable, pendant l'ardeur du soleil, sous des masses de fucus natans, dont elles embrassaient les folioles avec leurs nageoires rapprochées. Suffisamment doués sous le rapport de la locomotion, dans ces deux puissantes membranes, les Ptéropodes ne présentent jamais d'organes locomoteurs secondaires, ou même de modifications dans ceux que nous venons de décrire, du moins on n'en connaît point encore d'exemple.

Les Gastéropodes sont, de tous les mollusques, ceux qui nous fournissent les plus curieuses observations sur l'organe locomoleur ou ses modifications; ils constituent la classe la plus nombreuse peut-être, et ils appartiennent en grande partie aux rivages de 
la mer. Cependant il en est qui ne vivent que daris l'eau douce; d'autres sur le sol même, et à l'air libre; quelques uns enfin dans la haute mer : ainsi il a fallu pour ces derniers, ou le secours d'organes secondaires propres à la natation, ou au moins une modification importante dans l'appareil principal, qui est destiné à la reptation. Cet appareil, que l'on est convenu de nommer pied, est un disque musculaire plus ou moins étendu, mais toujours plus long que large, garni d'un grand nombre de fibres longitudinales, revêtu d'une peau quelquefois assez épaisse, et même calleuse sur les bords, et toujours située à la partie abdominale des mollusques, soit dans toute l'étendue du corps, comme chez les Limaces, les Aplysies, etc.; soit dans la partie antérieure seulement, d'où elle s'étend ensuite librement en arrière, tandis que la masse des viscères contournée en spirale, prend une autre direction, et va remplir la cavité d'une coquille, comme dans les animaux des Hélices, des Troques, etc. (I). Les Gastéropodes nus, à coquille interne ou sans coquille (2), et un petit nombre de ceux qui présentént une coquille externe, sont dans le premier cas, et le plus grand nombre de ceux qui sont pourvus d'une coquille externe sont dans le second. Au moyen de ce pied, ces mollusques rampent. "Mais cette sorte de " reptation, dit M. de Blainville, ne ressemble nulle" ment à la reptation des reptiles; c'est plutôt une " sorte de glissement du pied, produit par des ondu" lations extrêmement fines de tous les petits faisceaux " longitudinaux qui le composent, et qui se succèdent " du premier au dernier, chacun étant alternative" ment point d'appui, ou point fixe pour le suivant."

(I) On nomme ceux-ci Trachélipodes.

(2) Le Gastéroptère, qui est un mollusque nu, est pourtant trachélipode. Sa masse viscérale n'est point contournée en spirale. 
D'après cette définition, on doit concevoir que ces animaux sont fort lents dans leur progression, et l'expérience prouve de mème que le raisonnement, qu'ils le sont d'autant plus que leur pied est plus court et plus élargi ; aussi les Limaces et les animaux des Hélices jouissent encore d'une certaine vitesse; mais il n'en est pas de même de ceux des Fissurelles, des Patelles, etc., qui semblent au contraire ne jamais changer de place, tant ils sont lents, et dont le pied fait plus souvent l'office d'une ventouse pour les attacher aux corps, que celui d'un pied propre à la reptation.

Il est cependant quelques mollusques dont la manière de ramper diffère sous quelques rapports de celle-ci; on peut les nommer mollusques arpenteurs : certains Cyclostomes, quelques Hélicines, sont de ce nombre. Leur pied est allongé et très voûté, de sorte qu'ils n'appuient d'abord que son extrémité antérieure, et aussitòt en rapprochent l'extrémité postérieure pour recommencer encore à porter la premiere en avant, et ainsi de suite. Les mollusques arpenteurs sont plus vifs que la plupart des autres Gastéropodes.

La reptation des mollusques s'opère sur la surface des corps, soit à l'air libre, soit dans les eaux douces ou salées; mais quelques uns de ces derniers jouissent en même temps de la faculté de ramper à la surface même des eaux, en se tenant dans une position renversée, de manière que le pied glisse contre une légère couche d'eau qui semble lui servir de point d'appui. On pense bien que ces animaux doivent ètre très légers: ce sont de petites Doris, des Eolides, les Lymnés, les Physes, etc.

Les organes locomoteurs secondaires, que présentent les mollusques Gastéropodes qui s'éloignent un peu des rivages, sont, comme dans les Céphalopodes, des espèces de nageoires, mais ces nageoires ne sont pas précisément disposées de la même manière. Quelques uns d'eux ne nagent que dans certaines cir- 
constances, et reviennent bientôt sur les rivages pour s'accoupler et y déposer leurs ceufs; tels sont plusicurs Aplysies, quelques Acères, etc. Le pied leur étant toujours nécessaire, il existe encore complétement ; rien n'est changé, ni dans sa forme, ni dans ses propriétés ; mais le manteau forme deux lobes, quelquefois très grands, qui s'étendent de chaque côté, s'élèvent et s'abaissent tour à tour pour frapper l'eau lorsque l'animal veut se porter au loin du rivage, ou qu'il croise sur son dos, lorsqu'au contraire il revient y ramper. Tels sont encore les Thétys, les Mélibées, qui émigrent quelquefois vers les hautes mers, emportées sur des fucus par les courans ou les orages; ceux-ci ont encore un pied très distinct, mais au lieu des lobes latéraux, ils sont munis en avant d'une vaste membrane qui forme un voile autour de leur bouche, comme nous avons déjà eu occasion de le dire; et c'est au moyen de cet organe très puissant pour la natation, et d'un mourement de contorsion dans tout le corps, que nous avons vu ces animaux, en s'agitant, s'élever à la surface des eaux. D'autres ne rampent jamais, n'habilant que la haute mer où ils errent continuellement. Chez eux, le pied ne pouvant plus être d'aucun usage, n'existe plus, ou bien ne laisse que de faibles traces, et les organes secondaires, toujours natatoires, présentent différentes formes. Quelquefois, conme dans les Ptérosomes, ils sont composés d'une senle membrane mince qui fait horizontalement tout le tour de l'animal, le soutient par l'étendue de sa surface, et le déplace par ses mouvemens. D'autres fois, comme dans les Glauques et les Briarés, ce sont des expansions branchifères étendues horizontalement de chaque côté, en nombre plus ou moins grand, mais toujours pair, et qui s'agitent avec beaucoup de len१eur; ou bien encore, comme dans les Gastéroptères, deux vastes lobes étendus de chaque côté du pied, et dans ce cas le mouvement est précipité, et, de même que tout l'animal, rappelle les Ptéropodes, dont les 
Gastéroptères ont aussi les mours. Tous ces animaux se tiennent dans un sens renversé.

Les mollusques, qui présentent les phénomènes les plus singuliers, sous le rapport de la locomotion, sont sans contredit les Janthines et le Litiope.

La Janthine est munie de deux petites expansions latérales, au moyen desquelles elle se meut sur l'eau, car elle est aussi pélagienne, et on ne la rencontre sur les rivages que lorsqu'elle y est jetée par la tempête, et alors elle $y$ périt. Mais ces deux espices de nageoires, qui peuvent bien la mouvoir, ne sont pas suffisantes pour la tenir suspendue sur les eaux. La nature a suppléé à ce défaut par un moyen dont nous n'avons aucun exemple parmi les mollusques, mais qu'elle a mis souvent en usage chez les Acaléphes, qui sont des animaux d'un ordre bien inférieur. Ce moyen consiste dans un groupe très serré de petites vésicules aériennes de substance subcartilaginense et diaphane, attaché à la partie postérieure du pied, et au moyen duquel elle flotte constamment au-dessus des eaux. (i)

Plusieurs savans naturalistes ont vu dans cette rnasse de vésicules hydrostatiques un vestige de l'opercule. En effet, ceite pièce manque dans la Janthine, et l'on peut croire que ce singulier organe en tient lieu en remplissant l'ouverture de la coquille lorsque l'animal s'y contracte; mais cette masse n'adhère point de la même manière, ni à l'endroit ordinaire de l'opercule, puisqu'au lieu d'être au-dessus de la partie postérieure du pied, c'est au-dessous.

(I) Cette grappe de vésicules a un autre usage que nous sommes les premiers à faire connaître : elle sert encore à recevoir les œufs du mollusque; nous en avons compté jusqu'à cent quatre-vingts, suspendus sur une même ligne, à la face inférieure de l'une d'elles. Ainsi donc sir Everad Home s'est trompé quand il a dit que les œufs de la Janthine étaient fixés autour de la coquille par une substance glaireuse. 
Le pied de l'animal de la Janthine est très distinct; il est court, et nous ne le croyons nullement propre à la reptation; on peut le diviser en deux parties, l'antérieure, qui est concave et sert peut-être à fixer l'animal aux corps flottans, en faisant l'office d'une ventouse, et la postérieure, qui est plane et reçoit l'extrémité de la grappe vésiculaire.

Le Litiope, que nous établissons en genre nouveau, d'après la connaissance de son animal, est remarquable par une particularité qui en fait un des mollusques les plus curieux à connaître. Il a un pied qui nous a paru complet, et au moyen duquel il rampe sur les feuilles des plantes marines errantes dans la haute mer; mais il lui arrive souvent de s'écarter à une certaine distance en dessous de ces plantes, en ayant la précaution de s'y tenir constamment attaché par un fil gélatineux, qui est sans doute le produit d'une sécrétion particulière, et qui lui sert à remonter sitôt que quelque chose vient l'effrayer, semblable en cela à ces insectes qui descendent de leur habitation en filant une soie à peine visible, et qui $\mathrm{y}$ remontent en se pomojant dessus.

Les modifications dans l'appareil locomoteur principal des Gastéropodes sont nombreuses. En première ligne se présente naturellement celle qui forme un des caractères distinctifs de l'ordre des Nucléobranches, et dont on ne comnaît encore l'application que dans trois genres, les Carinaires, les wiroles et les Atlantes. Ces animaux sont uniquement pélagiens comme les derniers dont nous venons de parler, et ils ne rampent jamais; aussi leur pied a-t-il subi une telle modification, qu'il devient lui-même propre à la natation. Sa forme est en effet tout opposée à celle qu'il montre ordinairement, c'est-à-dire qu'au lieu de présenter une surface plane et horizontale, il s'élève dans une direction verticale, ayant la forme d'une nageoire comprimée et arrondie, à laquelle des faisceaux de muscles impriment un mouvement de 
droite et de gauche dont l'effet suffit pour la translation du mollusque. Cette nageoire occupe la partie médiane et ventrale; mais elle se trouve toujours supérieure, parce que ces animaux, comme tous les mollusques pélagiens, sont obligés de se tenir dans une position renversée.

Il arrive quelquefois, dans les Carinaires et les Firoles seulement, que cette nageoire ventrale est secondée par quelques autres membranes natatoires placées sur le cou ou à la partie caudale, mais dont la puissance n'égale cependant pas la sienne. Au moyen de ce système d'organes locomoteurs, ces animaux acquièrent une vitesse qui est la plus grande que nous ayons observée parmi les mollusques, car elle surpasse de beaucoup celle des Seiches. Les Carinaires et les Firoles surtout sont remarquables sous ce rapport. Jamais mous ne les avons vues à la surface de la mer, le dos en haut, et nous pensons qu'elles ne prennent cette position que lorsqu'elles tombent dans un état de souffrance; mais elles peuvent, avec la même facilité, et sans perdre de leur vitesse, se porter en arrière tout aussi bien qu'en avant, dans une direction courbe comme dans une direction droite. Il n'en est pas de même des Atlantes, dont tout le corps, ramassé en'spirale dans le petit espace de leur coquille, ne présente point la même résistance à l'élément qui les environne. Les Atlantes ne sont mues que par leur nageoire ventrale, qui est très grande, et il en résulte que tous leurs mouvemens sont, comme dans les Hyales, une sorte de sautillement vague et précipité.

Si ces animaux ne jouissent pas de la faculté de ramper, ils ont cependant celle de se fixer momentanément aux corps flottans, comme quelques Ptéropodes, mais par un moyen tout-à-fait différent, et qui consiste dans le secours d'une petite poche formée sur le bord supérieur et postérieur de la nageoire ventrale, par une sorte de dédoublement de la mem- 
brane qui la revêt. Cet organe, qui est évidemment le dernier vestige du pied du Gastéropode, et qui en a presque encore la forme, s'applique comme une ventouse à la surface des feuilles des plantes marines, tandis que le mollusque agit sur elle au moyen des muscles qui s'y rendent. Rien n'est plus ingénieux que celte disposition de l'appareil locomoteur des Nucléobranches, et nous sommes parvenus à nous convaincre de l'usage de cette ventouse par l'observation à l'état de vie des trois genres qui composent cet ordre, particulièrement des Atlantes, que nous avons fréquemment trouvés ainsi suspendus sous les plantes errantes.

Certains mollusques que l'on rencontre encore sur ces plantes marines, que quelques uns d'eux n'abandonnent même jamais, présentent d'autres modifications dans l'appareil locomoteur principal. De ce nombre sont les Scyllées, les Mélibées, et généralement les Aplysies de notre sous-genre Notarche. Ces animaux rampent peu; ils glissent très lentement sur les tiges ou les feuilles des plantes, et leur pied, pour mieux les saisir, est long et étroit, comme canaliculé. Daus le Notarche gélatineux, où il est très mince, et muni, dans son milieu, d'un étroit sillon, il présente en avant un petit appareil qui rappelle la ventouse des Nucléobranches, et sert probablement au même usage.

Nous pouvons citer encore parmi les modifications du pied, celle que l'on remarque dans le Pietin d'Adanson. Cet organe y est divisé, à ce qu'il paraît, en deux talons, par un enfoncement transversal, et il est probable, d'après cela, que c'est un mollusque arpenteur.

Le genre Hipponice doit également présenter une disposition particulière dans le pied, puisque ce mollusque est fixe, et qu'alors cet organe lui devient inutile. L'Hipponice et peut-être le genre Cabochon présentent les seuls exemples de Gastéropodes fixes. 
Les classes qui suivent celle des Gastéropodes ne sont pas, à beaucoup près, aussi généreusement doués sous les rapports des organes locomnteurs. Dans la classe des Acéphales, nous verrons, dès la première section, les organes locomoteurs disparaitre entièrement dans plusieurs genres. En rffet, dans cette section, qui comprend les Acéphales testacés, le plus grand nombre de mollusques est libre, et le reste est fixe; mais dans ceux-ci, on peut distinguer encore ceux qui, quoique liés anx corps par une production particulière des muscles, jouissent encore d'une certaine locomotion; et ceux qui, fixés immédiatement par leur enveloppe testacée, en sont entièrement privés. On conçoit que ce sont ces derniers qui ne montrent aucun organe locomoteur.

L'appareil locomotetir principal des Acéphales testacés porte aussi le nom de pied; mais il est tout différent du pied des Gastéropodes, et son usage n'est pas le même (r). C'est une masse musculaire, souvent assez épaisse, attach ì à la partie abdominale et médiane du mollusque, et plus ou moins en avant; elle est mue par un grand nombre de fibres, aux moyen desquels elle peut s'allonger et se contracter, se plier ou s'étenảre, enfin se diriger dans différens sens, mais particulièrement en avant. Pour se mouvoir sur le fond, le mollusque appuie contre le sol l'extrémité de ce pied, et l'allonge tout à coup pour

(x) On prétend, il est vrai, que la Psammobie orangée rampo à la manière des Gastéropodes, ses deux valves étalées de chaque côté du dos; mais ce fait, que nous ne contesions pas, n'est pas suffisamment prouvé; et l'animal de la Nucule, que M. de Blainville croit être dans le même cas, n'a fourni à ce savant une telle supposition que d'après la disposition de son pied. Si cela était ainsi, il y aurait évidemment un passage des Gastéropodes aux Acéphales testacés par les Patelles, d'une part, et la Nucule de l'autre. 
se pousser; ou bien il l'appuie plié, et le détend tout à coup comme un ressort. Dans tous les cas, c'est donc comme levier que ces animaux s'en servent, mais jamais comme organe de reptation.

Il n'y a point d'organe locomoteur secondaire chez les mollusques de cette section, à moins que l'on ne regarde comme tels les valves de quelques uns d'eux; car ils s'en servent avantageusement pour se mouvoir sur le fond, ou s'en élever, en les écartant et les rapprochant alternativement et vivement.

Les modifications dans l'organe principal sont assez nombreuses, mais peu importantes en général. "Ce pied extensible, dit M. de Blainville, ressemble " quelquefois à une sorte de ventouse, comme dans - les Nucules; à une espèce de langue, comme dans "les Moules, où il est canaliculé en arrière; à une " hache, comme dans les Vénus; à une sorte de pied " humain, comme dans les Cames; à une espèce de "fouet, comme dans les Loripèdes ". Il faut ajouter encore qu'il est quelquefois en forme de cône droit, comme dans les Solens; et cette dernière modification est particulièrement affectée à des mollusques qui s'enfoncent dans le sable au moyen de cet organe.

Les Acéphales testacés fixes peuvent se diviser en deux séries; l'une, qui renferme ceux qui, comme les Moules et les Jambonneaux, se fixent au moyen d'un byssus plus ou moins long, et conservent encore une certaine locomobilité, au moyen de laquelle ils se mettent dans différens rapports avec les corps environnans; et l'autre qui comprend ceux qui, à l'exemple des Huîtres, des Spondyles, etc., adhèrent immédiatement par une de leurs valves, et sont par conséquent complétement fixes. Les mollusques de cette première série montrent encore un pied, mais ceux de la seconde en sont privés.

Quant à la section des Acéphales sans coquilles, elle se compose de deux familles, dont l'une est entièrement fixe, comme les Ascidies, les Botrylles; et 
l'autre complétement libre, comme les Biphores. Ici la locomotion se fait par une organisation nouvelle, et.qui ne trouve d'exemple que dans la classe des Acaléphes, parmi les Diphies et les Béroés. Le corps de ces mollusques, enveloppé d'un vaste manteau, présente un large canal ouvert aux deux extrémités; l'élément ambiant le traverse, et c'est par l'action de diastole et de systole des lèvres de l'une de ces ouvertures que l'animal, chassant l'eau, s'imprime le mouvement, qui généralement est assez rapide. Ces mollusques, qui composent la famille des Salpiens, sont pélagiens; aussi ne les trouve-t-on que dans les hautes mers, et souvent réunis en nombre considérable, et de différente manière, mais toujours de telle sorte que les lèvres locomotrices soient en mesure de remplir leurs fonctions, ce qui s'opère à la fois pour tout l'ensemble de cette société.

La dernière classe, celle des Cirripèdes, ne présente plus de locomotion. Les animaux qui la composent sont tous fixes, les uns immédiatement par la base de leur coquille, les autres par un pédoncule tendineux, au moyen duquel ils peuvent cependant s'approcher ou s'éloigner un peu du corps sur lequel ils sont fixés; mais dans le nombre de ces mollusques, il en est qui suppléent en partie à ce défaut de locomobilité, en se fixant sur des corps errans, souvent même animés, et, par ce moyen, émigrent des lieux de leur naissance; telles sont, pour ceux qui se fixent immédiatement, certaines Coronules et les Tubicinelles qui s'introduisent dans la peau des cétacés; et pour ceux qui s'attachent à l'aide d'un pédoncule, quelques Anatifs, que l'on trouve sur le fucus natans, sur les coquilles des Janthines, et enfin le genre Alèpe, dont l'unique espèce n'a encore été rencontrée que sur l'ombrelle des Méduses.

Ainsi donc, dans la série méthodique décroissante de M. Cuvier, la locomotion et ses organes suivent pareillement une marche décroissante, depuis les premiers jusqu'aux derniers échelons. 
Outre l'orifice buccal, les mollusques en présentent plusieurs autres à la surface de leur corps; tels sont ceux des organes de la génération, de l'anus et de la cavité respiratrice. Quelquefois les orifices de la géné: ration sont cachés dans cette dernière; d'autres fois ils sont entièrement à découvert. Les mollusques qui ont les sexes séparés sur des individus différens, présenterit un exemple du premier cas dans tous les Céphalopodes, et un exemple du second dans les Pulmonés operculés et une partie des Pectinibranches. Dans ce dernier ordre, l'orifice de l'individu femelle est presque toujours situé à l'entrée de la cavité branchiale, ou dans cette cavité même, et celui de l'individu mâle, sur un organe excitateur contractile, qui se réfléchit généralement dans cette cavité.

Les mollusques qui ont les deux sexes sur le même individu, comme les Ptéropodes, les Nucléobranches, Nudibranches, Inférobranches, Tectibranches et Pulmonés, sans opercule, ont les orifices distans corrme les Tectibranches, ou réunis en un seul, comme les Inférobranches, etc.; mais, dans lous ces animaux, ces organes sont situés au côté droit, excepté cependant dans quelques cas anormaux, comme dans les Physes, les Planorbes, qui sont complétement sénestres.

Les mollusques qui ne jouissent que d'un sexe, le sexe femelle, comme les Scutibranches, Cirrhobranches, Cyclobranches, et tous les Acépales et Cirripèdes, n'ont qu' un seul orifice, celui des wufs, qui se trouve différemment placé. Dans les Scutibranches et les Cyclobranches, il est généralement au côté droit, près des tentacules. Dans les Acéphales, on ne connaît pas bien encore sa position, et dans les Cirripèdes, il est à l'extrémité du long tube qui s'élève au milieu des bras articulés. Du reste, l'orifice de l'anus ne varie pas moins, et se trouve également caché dans la cavité respiratrice, ou à découvert sur quelque partie du corps. Dans les Céphalopodes, où il est ca- 
ché dans la cavité branchiale, il est médian à la partie dorsale; dans les Ptéropodes, il est généralement au côté droit antérieur du manteau; dans les Gastéropodes, sa position varie beaucoup : par exemple, il est ouvert en entonnoir à l'extrémité d'un long tube arqué, placé au côté droit, chez les Atlantes; sessile et plus en arrière du même côté, chez les Glauques, Eolides, etc.; médian, dorsal, plus ou moins postérieur, au sommet d'un petit tube et au centre des arbuscules branchiaux, chez les Doris, Polycères, etc.; médian, mais à la partie postérieure et inférieure du rebord du manteau, chez les Onchidores; au côté gauche, chez le Planorbe et la Physe, mais, dans ces animaux, tous les autres organes sont ainsi transposés ; au côté droit en arrière des branchies, et sur un petit tube, chez les Pleurobranches; en arrière et à droite, à l'intérieur d'une sorte de syphon conduisant l'eau aux branchies, et formé par un repli de la membrane de l'opercule, chez les Aplysies; à l'extrémité postérieure et droite, chez la Testacelle; en avant et sur le collier, chez l'Hélice; toujours à droite et en avant dans les Pectinibranches; à gauche et dans la cavité branchiale, vis-à-vis son ouverture, chez. les Haliotides; à l'extrémité d'un petit tube flottant dans cette même cavité, chez les Calyptrées, Parmophores, Fissurelles, etc.; à l'extrémité postérieure de la ligne médiane, dans les Cirrhobranches; un peu à droite et au-dessus de la tête, chez les Patelles; sous l'extrémité postérieure, chez les Oscabrions; dans les Acéphales, il varie un peu moins : par exemple, il est en avant dans les Brachiopodes; en arrière et sur la ligne médiane; dans les Lamellibranches, dont les uns le présentent à l'extérieur comme l'animal des huîtres, et les autres à l'intếrieur du manteau, vis-àvis une ouverture ou un tube ouvert eu dehors, comme dans les Pyloridés; enfin, on le trouve à la base du long tube qui s'élève du milieu des bras articulés dans les Cirripèdes. 
La considération de ces différens orifices sexuels ou excrémentitiels est toujours d'une grande importance dans la distinction des genres, et celle de l'orifice de la cavité respiratrice l'est encore quelquefois davantage.

L'orifice de cette cavité n'existe que dans les mollusques chez qui l'organe de la respiration est caché, et alors il se montre, soit en forme d'ouverture arrondie ou de fente plus ou moins grande, soit à l'extrémité d'un tube. Son usage, dans tous les cas, est de donner passage à l'eau ou à l'air nécessaire à la respiration. Dans les Céphalopodes, l'orifice de la cavité respiratrice est à l'extrémité d'un tube conique et médian, se portant sous le cou, à l'entrée du sac. Dans quelques Ptéropodes, les branchies sont cachées, et, dans ce cas, le manteau s'ouvre de chaque côté en avant. Dans les Gastéropodes, elles le sont au contraire très souvent, et la cavité qui les contient s'ouvre à l'extérieur, soit par un petit orifice arrondi différemment situé au côté droit, comme dans les Limaces, Hélices, Parmacelles, etc.; soit par une grande solution de continuité à la partie antérieure et supérieure du manteau, comme dans les Pectinibranches; soit à la partie antérieure et sénestre, comme dans quelques Scutibranches.

Dans les Acéphales et les Cirripèdes, les branchies étant presque toujours cachées dans le manteau, elles reçoivent l'eau par une ouverture plus ou moins longue, ou par un tube qui leur correspond en arrière, et qui est terminé par un assez petit orifice souvent bordé de cirrhes tentaculaires.

Dans un grand nombre de mollusques Gastéropodes, Pectinibranches, une expansion particulière du manteau, que l'on désigne sous le nom de siphon ou de trompe, se porte en avant de la cavité branchiale, en passant par une échancrure ou un canal de la coquille, et ouvre de cette manière un passage à l'eau nécessaire aux branchies. 
La considération des organes de la respiration est d'une grande importance dans l'étude des mollusques, parce qu'ils fournissent d'excellens caractères pour l'établissement de la plupart des ordres et pour la distinction des genres. Ces organes sont de deux sortes, les uns pulmonaires, aquatiques ou aériens; les autres branchiaux et toujours aquatiques. Dans le premier cas, c'est simplement une cavité étendue sur la partie antérieure et supérieure du mollusque, s'ouvrant sous le bord du manteau par un orifice arrondi, susceptible de dilatation et de contraction, à la volonté de l'animal. Cette cavité est tapissée d'un réseau de vaisseaux pulmonaires qui rampent sur ses parois, et particulièrement à son plafond. Au moyen de cet appareil, les mollusques qui en sont doués respirent l'air libre comme les Limaces, les Scarabes, etc.; ou l'eau douce, comme les Planorbes, les Physes, etc.; ou l'eau salée, comme les Auricules. Les premiers rampent sur le sol, surtout dans les lieux humides; les seconds vivent à de petites profondeurs dans les rivières et les étangs, parce qu'ils sont obligés de venir fréquemment respirer à la surface de l'eau; et les troisièmes sur les bords des rivages, dont ils s'éloignent souvent de plusieurs toises pour venir ramper à l'air libre. Cet appareil respiratoire est le caractère principal des deux ordres des Pulmonés operculés et des Pulmonés inoperculés, qui appartiennent à la classe des Gastéropodes.

Dans le second cas, c'est-à-dire dans celui qui comprend les organes respiratoires branchiaux, on trouve une variété de conformation et de disposition qui n'existait point dans le premier; aussi en tire-t-on un grand avantage pour la classification. Il arrive même quelquefois que les branchies, si nécessaires à la vie des mollusques, se lient à d'autres organes remplissant d'autres fonctions, ou bien qu'elles-mêmes en remplissent d'autres tout-à-fait secondaires. Ces cas 
sont rares; cependant ils existent dans quelques Ptéropodes, par exemple, où elles tapissent les nageoires, comme dans les Clios, les Psychés, ou dans les Glauques, où, découpées en lanières et réunies en bouquets de chaque côté du corps, elles présentent un grand nombre de petites rames, et par ce moyen servent elles-mêmes à la locomotion; ou bien encore, comme cela parait devoir être dans plusieurs mollusques Lamellibranches, ces organes peuvent servir à retenir les aufs pendant quelque temps, après la ponte.

Les branchies, comme nous l'avons vu, sont souvent cachées dans une cavité particulière formée par le manteau. Cette organisation a lieu dans les Pectinibranches parmi les céphalés el daus tous les acéphalés; mais très souvent aussi ils ne sont protégés que par une sorte d'opercule, comme dans les Aplysies; ou simplement par des expansions du manteau, comme dans les Placobranches, ou par le rebord même de ce manteau, comme dans les Pleurobranches; ou enfin ils sont entièrement nus sur quelque partie de la surface du corps, comme dans les Nudibranches. Dans ce dernier cas, les branchies semblent être des ornemens qui rendent ces animaux d'un aspect fort agréable à l'oil. Nous allons les examiner sous le rapport de la siructure, de la forme et de la position, afin de mieux faire sentir l'importance de ces organes dans l'étude des mollusques.

Les branchies paraissent être en général le parłage de ceux qui vivent à d'assez grandes profondeurs, et qui par conséquent ne respirent point habituellensent l'air libre. La nature, en conformant ces organes, semble avoir chcrché à étaler dans le plus petit espace possible le plus grand nombre de vaisseaux propres à la respiration, afin qu'ils pussent plus facilement entrer en contact avec la quantité d'air nécessaire à cette fonction, dans un milieu où il est si rare. En effet, elles sont formées de lamelles, de 
feuillets, de ramifications, etc., toutes propres à augmenter les surfaces qui doivent recevoir le réseau. vasculaire, organisation qui nous rappelle celle des végétaux, dont les feuilles, multipliées à l'infini, présentent au contact de l'air leurs doubles surfaces, afin d'y recevoir avec abondance les élémens nécessaires à leur existence.

Quand on examine les branchies à l'aide du microscope, on y découvre un nombre considérable de vaisseaux, souvent rangés dans un ordre régulier, formant des anastomoses multipliés qui échappent bientôt à l'œil, et ressemblent à ces mêmes vaisseaux rampant à la surface interne des cavités respiratrices dans les Pulmonés. La différence qui existe entre les branchies et ces cavités est donc de peu de valeur, physiologiquement parlant, car elle se borne à celle du plus ou moins de surface propre à recevoir les ramifications capillaires des vaisseaux artériels, et semble provenir de ce que les uns sont destinés à plonger dans un fluide peu chargé d'air, et qu'il leur faut par conséquent plus de moyens pour le rassembler, tandis que les autres reçoivent cet air plus complétement libre.

Les Céphalopodes portent leurs branchies cachées dans une poche dorsale, ou du moins on ne connaît point encore, parmi ces animaux, d'exemple du contraire. Elles sont paires, symétriques et composées d'une ou de deux rangées de feuillets pédiculés, divisés et subdivisés.

Les Ptéropodes ne sont point encore tous connus sous le rapport des organes de la respiration, et l'on trouve des différences notables parmi ceux qui le sont. Ainsi, les Clios, les Psychés, et sans doute les Cymbulies, présentent leurs branchies en forme de réseau vasculaire, divisé par séries, et tapissant la surface des nageoires; les Hyales les portent cachées dans le manteau et en forme de peigne, sur une ligne arquée, des deux côtés, probablement, quoique M. de Blain- 
ville n'en ait encore fait l'observation que du côté droit. Les Cuviéries les présentent au nombre de deux, égales et symétriques, contournées de diverses manières, amincies sur un de leurs bords, ondulées et fixées sur un même pédicule, non par leur extrémité, mais par un point un peu distant de l'une des extrémités; elles suivent les mouvemens de l'animal et se portent au-dehors de la coquille lorsqu'il nage, ou bien y rentrent avec lui. Les Pneumodermes les ont de formes plus extraordinaires; elles sont placées, dans ces animaux, qui sont nus, à la surface extérieure du corps, tout près de l'extrémité postérieure : leur forme est celle de deux $\mathrm{C}$ adossés de cette manière $\mathrm{D} \mathrm{C}_{\text {, }}$ et réunies par deux petites barres tranverses. Elles sont en outre garnies de chaque côté d'un nombre assez considérable de petites folioles saillantes et régulières.

Les Gastéropodes présentent plus que toutes les autres classes de grandes différences dans la structure, la forme et la position des organes de la respiration; aussi s'est-on servi des caractères qui en résultent pour l'établissement de divers ordres. Le premier de ces ordres, celui des Nucléobranches, montre des branchies en forme de peigne, dont chaque pointe est divisée en feuillets; elles sont situées à la partie dorsale, plus ou moins en arrière ou en avant, et se groupent souvent avec d'autres viscères, de manière à former un nucléus que recrouvre toujours une portion du manteau ou une coquille. Les Nudibranches les portent toujours à nu, sur quelques parties du corps, et disposées symétriquement; elles sont de chaque côté, comme dans les Glauques, les Briarées et les Scyllées, sur toute la longueur du dos, comme dans les Eolides, Cavolines, Tergipes, Thétys, Mélibées et Tritonies; en un seul groupe sur un point de la ligne médiane, plus ou moins postérieur et dorsal, comme dans les Polycères, Doris et Onchidores. La forme de ces branchies varie encore dayantage: ce sont 
des lanières coniques réunies en éventail autour d'un nombre variable de petites expansions arrondies du manteau, comme dans les Glauques; de petites houppes hérissant la surface de pareilles expansions, comme dans les Scyllées; des cirrhes coniques, lamelliformes ou écailleux et nombreux, comme dans les Eolides, disposés par rangées transverses, dans les Eolides proprement dites, ou par séries longitudinales, dans les Cavolines; deux séries longitudinales de houppes rameuses, alternativement inégales de droite à gauche, ou de l'avant à l'arrière, comme dans les Thétys; deux séries peu nombreuses de massues oblongues, pédiculées à leur base, et recouvertes de petits tubercules hispides, comme dans les Mélibées; ou enfin un groupe d'arbuscules plus ou moins déliés et ramifiés, régulièrement étalés autour de l'anus, comme dans les Doris, Polycères, etc. Les Inférobranches portent les branchies à la partie inférieure du corps, entre le rebord avancé du manteau et celui du pied, soit tout autour, comme dans les Phyllidies, soit au côté droit seulement, comme dans les Pleurobranches, et ces organes ont la forme d'un long cordon ou d'un panache composé d'une série simple ou double de lamelles obliques, serrées les unes contre les autres. Les Tectibranches les portent sur le dos, mais toujours protégées par une ou deux expansions du manteau, et souvent recouvertes par une coquille rudimentaire ou complète, interne ou externe. Dans les Aplysiens et les Acères, elles sont en forme de panache comprimé et étalé en éventail, plus ou moins découpé et prolongé postérieurement. Les Pectinibranches, qui forment l'ordre le plus nombreux des Gastéropodes, portent tous les branchies attachées au plafond d'une cavité particulière, située au-dessus de la partie antérieure du dos du mollusque, et elles ont la forme d'un peigne subdivisé lui-même dans chacune de ses branches. Les Scutibranches les ont dans une cavité particulière, 
soit sur le dos, soit à gauche entre le bord du manteau, et elles ont la forme de deux peignes inégaux, comme dans les Haliotides, ou égaux, comme dans les Parmophores, attachés de chaque côté; ou bien de lamelles ou filamens adhérens au plancher de cette cavité, et souvent exsertiles, comme dans les Calyptrées, etc. Les Cirrhobranches, qui ne comprennent encore que le genre Dentale, portent les branchies sur deux lobes radicaux, situés au-dessus du cou, et elles sont en forme de filamens longs et très multipliés. Enfin, les Cyclobranches, qui composent le dernier ordre des Gastéropodes, portent les branchies à peu près comme certains Inférobranches, autour du corps, sous le rebord du manteau, et en forme de cordon plus ou moins complet, composé de pyramides ou de feuillets : tels sont les Patelles et les Oscabrions.

La classe des Acéphales présente aussi quelque variété dans les branchies. Tous les mollusques qui la composent ont ces organes en dedans du manteau; mais chez les Brachiopodes, qui en forment le premier ordre, ils sont appliqués à la face interne de chacun de ses lobes, et pectinés. Les Lamellibranches offrent tous deux paires de branchies, une de chaque côté du corps, c'est-à-dire entre le corps et le manteau, mais plus ou moins réunies à leur partie supérieure et postérieure, dans la ligne médiane; quelquefois même elles sont libres dans cette partie. Ces branchies, souvent inégales d'un même côté, sont en forme de lames semi-circulaires, et se montrent finement et régulièrement striées dans le sens de la hauteur, et quelquefois ponctuées dans les intervalles des stries. Les Hétérobranches présentent une disposition différente dans les hranchies, c'est-à-dire qu'elles ne sont, dans aucun cas, divisées en quatre lames, comme dans l'ordre précédent. Du reste, elles ne sont pas les mêmes dans tous les genres; dans la famille des Ascidiens, par exemple, c'est un 
réseau à mailles quadrangulaires, tapissant la cavité du tube excrémentitiel jusqu'à la bouche; dans les Salpiens, c'est une écharpe allongée, mince, divisée par petites locules régulières et transverses, étendue obliquement de l'extrémité antérieure à l'extrémité postérieure, comme dans les Biphores.

La dernière classe des mollusques, celle des Cirripèdes, offre peu de sujets d'observation sur les branchies, parce qu'elles ne sont pas encore suffisamment connues. Dans la famille des Lépadiens, elles sont attachées en dehors et à la base des cirrhes, par conséquent à l'intérieur du manteau; elles se montrent en forme de pyramides plus ou moins nombreuses. Enfin, dans les Balanides, elles paraissent aussi attachées à la face interne du manteau, et sont en forme d'ailes frangées.

D'après cette revue rapide des organes respiratoires des mollusques, on voit qu'il reste peu de chose à connaître sur cette partie si importante de leur organisation; cependant il existe encore des doutes dans certains cas. Par exemple, il n'est pas bien prouvé que les Clios, Psychés et Cymbulies respirent au moyen du réseau vasculaire que l'on croit apercevoir sur leurs nageoires; la Patelle offre encore sujet à controverse, car depuis que M. Cuvier a cru reconnaître les branchies dans le cordon de feuillets qui entoure leur corps entre le manteau et le pied, MI. de Blainville a pensé qu'au contraire ces animaux respiraient à la manière des Pulmonés, c'est-à-dire par le moyen d'une cavité tapissée d'un réseau vasculaire, question qu'il serait bien essentiel de résoudre. Enfin, quelques auteurs, Méry, Bojanus et Raspail, ont élevé des doutes au sujet des branchies des Lamellibranches, ne voyant, dans ces deux paires de lames latérales, que des organes appartenant à la génération. Plusieurs genres, comme on le verra dans notre genera, n'ont point encore montré ces organes importans.

Après avoir décrit rapidement les organes exté- 
rieurs des mollusques, nous jetterons un coup d'œil non moins rapide sur quelques unes de leurs facultés, sur ce qu'ils présentent d'utile ou de nuisible à l'homme; et enfin nous dirons un mot de leur genre d'habitation.

Les mollusques sont en général grandement doués sous le rapport du sens du toucher, et très peu sous celui des autres. Le toucher n'a pas seulement son siége dans quelques organes qui paraissent lui être spécialement affectés, il l'a encore sur tous les points de la surface du manteau, et l'on voit en effet que celui-ci est d'une sensibilité extrême, et d'autant plus grande, qu'il est moins épais et consistant. Les organes, qui sont en outre destinés à seconder cette sensation générale, sont les tentacules, dans un grand nombre, des expansions filiformes des bords du man. teau, dans quelques uns, et des cirrhes ou des papilles tentaculaires, dans beaucoup d'autres; on peut sans doute citer encore parmi eux ces expansions simples ou rameuses que l'on remarque sur la surface du manteau de certains mollusques, quelques Aplysies et Notarches, par exemple, et même ces tubercules et ces aspérités qui se montrent sur celui de quelques Doris ou Pleurobranches, et de toutes les Carinaires.

La vision n'appartient qu'aux trois premières classes des mollusques, encore est-il fort incertain qu'elle existe chez les Ptéropodes. Très étendue chez les Céphalopodes, elle devient souvent bornée chez les Gastéropodes, comme il est permis d'en juger par son organe qui, généralement, est imparfait, et ne présente même que des traces assez douteuses.

Nous sommes encore moins instruits sur le sens du goût. M. de Blainville, remarquant que les sensations spéciales sont souvent en rapport inverse de développement avec la sensation générale du toucher, en conclut que probablement le sens du goût doit être nul dans les Acéphales, et que selon toute apparence il n'est pas très fir dans les autres. Ce sa. 
vant pense qu'il en est de même pour l'odorat, c'està-dire que les mollusques céphalés seuls jouissent de ce sens, et il paraît porté à croire que son siége es氏 placé dans la première paire de tentacules. L'expérience prouve en effet que quelques uns de ces animaux discernent parfaitement, pendant la nuit, les plantes dont ils font de préférence leur nourriture.

La sensibilité exquise du manteau des mollusques rendant, jusqu'à un certain point, inutile chez eux l'audition, puisque la transmission du bruit peut leur être faite par la secousse qu'il produit, ils en sont presque tous privés. Le Poulpe et la Seiche sont les seules, d'après nos connaissances actuelles, qui en jouissent; mais il en est sans doute d'autres encore qui partagent avec eux cet avantage.

Après l'examen que nous avons fait des organes extérieurs des mollusques, on doit concevoir que ce sont des êtres faibles, incapables d'attaguer, et très imparfaitement munis de moyens pour se défendre des animaux marins qui les recherchent pour en faire leur pâture; cependant on peut signaler, comme moyen de défense, cette faculté de se contracter, soit dans l'intérieur d'une enveloppe testacée, soit dans des cavités propres à leur servir d'abri. La fuite ne peut en être un pour eux, car ils sont généralement d'une lenteur remarquable, si on en excepte les Ptéropodes, les Nucléobranches, les Seiches, et particulièrement les Calmars qui, à la moindre approche, s'élancent quelquefois à d'assez grandes hauteurs. Il est encore un moyen de défense assez ingénieux, mais que l'on ne trouve que chez un petit nombre de mol. lusques; il consiste dans une liqueur généralement désagréable par son odeur, souvent colorée, dont ils sont munis, et qu'ils épanchent autour d'eux lorsqu'ils sont inquiétés, de manière à former un petit nuage protecteur qui ne les soustrait pas seulement à la vue de leur ennemi, mais qui sans doute repousse celui-ci par une propriété qui est particulière à cette 
liqueur. Ainsi l'Aplysie, sur les rochers, et quelques autres encore, répandent autour d'eux une liqueur pourprée, en même temps que la surface de leur corps se recouvre d'une couche épaisse d'un mucus incolore non moins abondant; la Seiche, nageant entre deux. eaux, déjette spontanément une liqueur noire qui trouble pour un moment l'élément qui l'environne; et dans les mers boréáles, les Clios, qui se rencontrent quelquefois en si grande abondance, s'enveloppent d'un petit nuage blanchâtre qui paraît s'échapper de toute la surface de leur corps. Les mollusques n'ont donc que des moyens secondaires pour se défendre, sans posséder jamais une arme appropriée à ce but.

Les services que ces animaux rendent à l'homme sont très nombreux. Beaucoup d'entre eux contribuent à sa nourriture, et sont pour la classe indigente, sur les bords de la mer, d'une grande ressource; il en est que les riches ne dédaignent point, et qui, dans beaucoup d'endroits, ajoutent au luxe de leur table. Par cette raison, le commerce y trouve une spéculation qui fait souvent en grande partie la fortune de tout une population. Outre ces avantages, les plus importans sans doute que nous puissions signaler, il en est beaucoup d'autres qui unéritent néanmoins d'être cités : la Seiche, par exemple, fournit, dans la liqueur noire qu'elle sécrète, une couleur recherchée pour la peinture, connue sous le nom de sépia, et celle plus répandue encore, dont les Chinois nous ont appris l'usage, et que l'on appelle, à cause de cela, encre de Chine. On ne donte pas que la pourpre des anciens ne fût le produit d'un mollusque; et si cette belle teinture est perdue pour notre industrie, ne peut-on espérer de la retrouver un jour dans les liqueurs si colorées de certaines Aplysies et surtout de la Janthine. Beaucoup de coquilles fraîches, ou même fossiles, servent à faire de la chaux, comme un Strombe, à la Martinique, le Concholepas, dans certains endroits de la côte du Pérou, et une espèce d'Huître fossile, au Sénégal. II 
est inutile de rappeler tous les secours que l'industrie a su tirer de la coquille des mollusques, tout le monde les connaît et admire entr'autres ces camés ciselés avec art, et que les graveurs tirent d'une espèce de Casque; cette nacre qui brille sur nos plus beaux meubles; et, par-dessus tout, cette production singulière d'une espèce d'Huitre, ces perles tant recherchées, qui forment un des plus précieux ornemens de la parure des femmes, et dont le brillant et la blancheur, loin de nuire à l'éclat d'une belle peau, ne servent qu'à lui prêter un nouveau charme. Nous signalerons un fait qui mérite sans doute une grande attention, parce qu'il se rattache à une branche de commerce importante. C'est à M. le capitaine de frégate Bellanger que nous sommes redevables de cette observation. Cherchant à approfondir les motifs qui font que nos pêcheurs de morue ne sont pas aussi heureux dans leurs opérations que les Américains, il découvrit que cela provenait de ce que ces derniers se servaient pour appât, de l'animal d'une Mye très abondante sur quelques points de la côte d'Ámérique, et il s'en convainquit davantage encore en remarquant que les Français, à la fin de la pêche des Américains, achetaient ce qui leur restait de ces animaux pour l'employer au même usage, et compléter plus promptement leur chargement. M. Bellanger, très instruit en conchyliologie, examina attentivement cette Mye, et la reconnut pour une espèce de France commune sur les côtes de la Manche. Cette observation mérite sans doute de fixer l'attention du commerce, car elle indique un moyen de rendre peut-être plus productive et moins dispendieuse une branche pénible à exploiter, continuellement soumise à de funestes chances, et généralement peu lucrative pour ceux qui s'y livrent.

Si maintenant nous jetons un coup d'œil sur le mal que causent les mollusques, nous ne trouvons que celui qui résulte des dégâts que quelques uns d'eux 
font, soit dans nos jardins, soit dans nos ports; et sous ce rapport leur étude est encore d'une grande utilité, puisqu'elle les signale et fait connaître leurs mœurs. Les Limaçons, les Limaces, les Testacelles et quelques autres mollusques terrestres, recherchent en effet les jardins potagers, et, par la facilité avec laquelle ils se multiplient, et leur voracité, détruisent quelquefois, en un court espace de temps, toutes les espérances du jardinier. Dans quelques colonies où ces animaux sont plus grands qu'en Europe, ils font quelquefois beaucoup de dégâts dans les plantations.

Quelques mollusques marins sont également dans le cas de causer de grands dommages, soit à la carène des navires, soit dans la construction des digues; ce sont particulièrement les Tarets, qui pénètrent dans le bois et le creusent dans tous les sens.

Nous terminerons par dire un mot sur l'habitation des mollusques. Ceux qui sont marins habitent, les uns sur les rivages, et les autres la haute mer. Les premiers se rencontrent souvent fixés aux corps sous-marins, découvrant par l'effet des marées : telles sont les Patelles, les Crépidules, les Huîtres, les Cames, les Ascidies, etc.; d'autres sont libres, comme les Buccins, les Casques, les Donaces, etc. : les uns habitent sur les rochers, les autres sur les fonds sablonneux ou vaseux, se recouvrant quelquefois d'une légère couche de sable ou de vase, comme les Bullées, ou s'enfonçant même dans le sol, comme font un grand nombre de coquilles bivalves, telles que les Solens, les Myes, Lutraires, Anodontes, etc. La profondeur à laquelle les mollusques peuvent vivre est assez grande, puisque nous avons obtenu, au moyen de la sonde, à 5 o à 55 brasses de profondeur, un petit mollusque vivant, appartenant au genre Fuseau. Quant à ceux qui sont Pélagiens, ils vivent très au large des côtes, et se tiennent à la surface de la mer lorsqu'elle est calme, ou à une profondeur d'autant 
plus grande qu'elle est plus agitée. Il est cependant de toute probabilité qu'ils ne s'approchent jamais du fond, car ils ne sont point organisés pour ramper. Les mollusques Pélagiens vaguent sans cesse; aussi il n'est pas extraordinaire de rencontrer les mêmes espèces dans des mers différentes et éloignées, sans égard même quelquefois pour le changement de climat.

Plusieurs mollusques marins vivent dans des eaux saumâtres et presque douces; les Potamides, par exemple, se rencontrent souvent à l'embouchure des fleuves, et nous avons trouvé à l'île de Bourbon, dans une marre d'eau presque complétement douce, mais peu éloignée du rivage, des Pintadines et des Aplysies ( $A$. Dolabrifera) vivant en société, sous les pierres, avec des Néritines ( $N$. Auriculata) et une Mélanie. Enfin, il arrive aussi quelquefois qu'ils respirent l'air libre, soit à la surface de l'eau, comme font les mollusques Pélagiens, soit sur les rochers, à quelques toises du rivage, comme nous l'avons vu faire aux Auricules particulièrement ( $A$. Myosotis), aux Littorines, aux Planaxes et à une espèce de bulle que nous venons de découvrị ( $B$. Haliotis) sur les rochers de cette île.

Les mollusques terrestres habitent les jardins, les bois, les champs, les rochers, et même les terrains arides et sablonneux. Il en est qui sont, pour ainsi dire, domestiques, car ils paraissent rechercher les habitations de l'homme. Ils se cachent dans la terre, sous les feuilles, dans les lieux humides, les fentes des rochers, ou même sur les arbres; et c'est surtout pendant la nuit ou dans les temps pluvieux qu'ils sortent de leur retraite. Quelques uns de ces a nimaux, tels que les Ambrettes, recherchent quelquefois le bord des eaux douces, mais elles ne sauraient vivre dans cet élément, non plus qu'aucun autre mollusque terrestre.

Quant aux mollusques fluviatiles, on les trouve dans les rivières, les lacs, les étangs et les fossés, 
mais jamais à de grandes profondeurs. Ils rampent sur le fond, ou sur les plantes aquatiques, ou bien, comme nous avons eu occasion de le dire, à la surface de l'eau. Plusieurs d'entre eux vieınent sur le bord respirer l'air libre, telles sont les Ampullaires, mais surtout cette curieuse espèce de Néritine, que les naturalistes de l'expédition de la Coquille ont trouvée en si grande quantité sur les arbres, à une grande distance des eaux douces.

Avant d'entrer en matière, nous allons faire connaître les différentes méthodes de classification employées par les naturalistes Français qui, depuis quelques années, se sont le plus occupés de l'étude des mollusques, ou seulement de leurs coquilles. Celle de M. de Férussac n'est qu'une modification de celle de M. Cuvier, nécessitée, à l'époque où elle a été faite, par de nombreuses découvertes; et l'on verra, dans notre genera, qu'il en est de même de celle que nous avons adoptée, car nous ne pensons pas que l'on puisse classer les mollusques d'une manière à la fois commode, facile et naturelle, si l'on s'écarte de la route que ce savant a tracée dans son règne animal. 


\section{MÉTHODE DE M. CUVIER. I 8I 7.}

DEUXIEME GRANDE DIVISION DU REGNE ANIMAL. LES MOLLUSQUES.

$$
\text { ire Classe. }
$$

\section{LES CÉPHALOPODES.}

Los seiches, poulpes, polypes d'Aristote, élédons, calmars, seiches.

Les nautilles, spirules, nautilles qui comprennent les ammonées, lenticulines, sphinctérules, pélores, géopones, chrysoles, théméones, lampadies, andromèdes, cellulies, noniones, mélonies, rotalies, éponides, polyxènes, tinopores, floriles, cibicides, storiles, éolides, discorbes, oréades, planulites, ellipsolites, amaltées, cancrides, pénéroples, astacoles, linthuries, scortimes, périples, Jituus, lituites, hortoles, spirolines, nodosaires, molosses, réophages, raphanistres, échidnes, téléboïtes; orthocératites.

Les bélemnites.

Les hippurites, qui comprennent les batholites.

Les ammonites, qui comprennent les ammonites, orbulites, baculites, turrilites.

Les camérines, camérines qui comprennent les sidérolithes, archidies, ilotes, hélénides, rénulites, mélonies, borélies, clausulies, miliolithes; milioles, pollontes, aréthuses.

Les argonautes.

$$
2 \text { e Crasse. }
$$

\section{LES PTÉROPODES.}

Téle distincte.

\section{İes clios.}

Les cléodores. 
Les cymbulies.

Les limacines.

Les pneumodermes.

Téte non distincte.

Les hyales.

3e Crasse.

\section{LES GASTÉROPODES.}

$$
\text { Ier ORDRE. }
$$

LFS NUDIBRANCHES.

Les doris. Les polycères.

Les tritonies.

Les thétys.

Les scyllées.

Les glaucus.

Les éolides.

Les tergipes.

2 ORDRE.

LES INFÉROBRANCHES.

Les phyllidies.

Les diphyllides.

$$
\text { 3e OrdRe. }
$$

\section{LES TECTIBRANCHES.}

Les pleurobranches.

Les aplysies.

Les dolabelles.

Les notarches.

Les acères, qui comprennent les bullées, bulles, acères. 
DE M. GUVIER .

$4^{\text {e OrdRE. }}$

LES PULMONÉS.

Pulmonés terrestres.

Les limaces, limaces, testacelles, parmacelles.

Les escargots, escargots, vitrines, bulimes, maillots, scarabes, grenailles, ambrettes.

Les nompareilles.

Les agatines, qui comprennent les agatines, liguus, polyphêmes.

Pulmonés aquatiques.

Les onchidies.

Les planorbes.

Les lymnées.

Les physes.

$L_{\text {es auricules. }}$

Les mélampes.

Les actéons.

Les pyramidelles.

5e ORDRE.

\section{LES PECTINIBRANCHES.}

Les Trochoĩdes.

Les sabots, sabots qui comprennent les méléagres et les turbos; dauphinules, vermets, turritelles, scalaires, cyclostomes, valvées, paludines, monodontes.

Les toupies, toupies, cadrans.

Les conchylies, ampullaires, mélanies, phasianelles, janthines.

Les nérites, natices, nérites, néritines.

\section{Les Buccinoindes.}

Les cornets.

Les porcelaines. 
Lés ovules, qui comprennent les ovules, navettes, calpurnes.

Les tarières.

Les volutes, olives, volutes qui comprennent les cymbes, volutes; marginelles qui comprennent les colombelles; mîtres, cancellaires.

Les buccins, buccins, éburnes, tonnes qui se divisent en tonnes et perdrix; harpes, nasses, pourpres, casques, heaumes, vis.

Les cérithes, cérithes, potamides.

Les rochers, murex qui comprennent les murex, brontes, typhis, chicoracés, aquiles, lotoires, tritons, trophones; ranelles qui comprennent les apolles; fuseaux qui comprennent les fuseaux, lathires, pleurotomes, pyrules, fasciolaires, carreaux; turbinelles.

Les strombes, strombes, ptérocères, rostellaires qui comprennent les hippocrènes.

Les Sigarets.

Les sigarets.

$$
\text { 6e OrdRe. }
$$

LES SCUTIBRANCHES.

Les ormiers, haliotides, padoles, stomates.

Les cabochons.

Les crépidules.

Les fissurelles.

Les émarginules.

Les septaires.

Les carinaires.

Les calyptrées.

7e ORdRE.

LES CYCLOBRANCHES.

Les patelles.

Les oscabrions. 
DE M. GUVIER.

$4^{e}$ Crasse.

\section{LES ACÉPHALES.}

I er ORDRE.

\section{ACÉPHALES TESTACÉS.}

\section{Les Ostracés.}

Les huitres, acardes, huîtres, gryphées, peignes, limes, houlettes.

Les anomies.

Les placunes.

Les spondyles, spondyles, plicatules.

Les marteaux.

Les vulselles.

Les pernes.

Les arondes.

Les crénatules.

Les jambonneaux.

Les arches, arches qui comprennent les cucullées; pétoncles, nucules.

Les trigonies.

\section{Les Myticacés.}

Les moules, les moules, modioles, lithodomes.

Les anodontes.

Les mulètes.

Les cardites.

Les vénéricardes.

Les crassatelles.

Les B hintiers.

Les tridacnes, tridacnes, hippope.

Les Cardiacés.

Les cames, cames, isocardes.

tes bucardes, qui comprennent les hémicardes. 
Les donaces.

Les cyclades.

Les corbeilles.

Les tellines.

Les loripèdes.

Les lucines.

Les vénus, vénus qui comprennent les cythérées; capses, pétricoles.

Les corbules.

Les mactres, mactres qui comprenment les lavignons.

\section{LEs ENFERMÉs.}

Les myes, lutraires, myes, anatines, glycymères, panopes, pandores.

Les gastrochénes.

Les byssomies.

Les hiatelles.

Les solens, solen, sanguinolaires.

Les pholades.

Les tarets.

Les fistulanes.

$2^{\text {e Ordre. }}$

LES ACÉPHALES SANS COQUIILES.

Antmaux isolés.

Les biphores.

Les ascidies.

Anima uX composés.

Les botrylles.

Les pyrosomes.

Les polyclines.

5e Classe.

LES BRACHIOPODES.

Les lingrules. 
Les térébratules.

Les orbicules.

$$
\begin{gathered}
\text { 6 C Classe. } \\
\text { LES GIRRHOPODES. }
\end{gathered}
$$

Les anatifes.

Les glands de mer, balanes, coronules, tubicinelles. 


\section{MÉTHODE DE M. DE LAMARCK. I 8 I 8 .}

\section{ANIMAUX SANS VERTÈBRES.}

Classe X.

LES CIRRIPÈDES.

ORDRE I.

\section{CIRRIPÈDESS SESSILES.}

(r) Opercule quadrivalve.

Tubicinelle, coronule, balane, acaste.

(2) Opercule bivalve.

Pyrgome, creusie.

ORDRE II.

CIRRIPÈDES PÉDONCULÉS.

(1) Corps incomplétement enveloppé par sa tunique; sa coquille, composée de pièces contiguës, laisse ⿳亠े l'animal une issue librs, lorsqu'elle s'ourre.

Anatife, pouce-pied.

(2) Corps tout-à-fait enveloppé par sa tunique, mais qui offre une ouverture antérieure. Sa coquille, formée de pièces séparées, n'a pas besoin de s'ouvrir pour la sortie des bras de l'animal.

Cinéras, otion. 


\section{Classe XI.}

\section{LES CONCHIFÈRES.}

\section{Ordre I.}

\section{CONCHIFÉRES DIMYAIRES.}

(1) Coquille régulière, le plus souvent équivalve.

(a) Coquille en général béante aux extrémités latérales, ses valves étant rapprochées.

(") Conchifères crassipèdes. 69 \$

Les tubicolées. Arrosoir, clavagelle, fistulane, cloisonnaire, térédine, taret.

Les pholadaires. Pholade, gastrochêne.

Les solénacées. Solen, panopée, glycymère.

Les myaires. Mye, anatine.

$\left({ }^{* *}\right)$ Conchifères ténuipèdes.

$(\dagger)$ Ligament intérieur, avec ou sans complication de ligament externe.

Les mactracées. Lutraire, mactre, crassatelle, érycine, onguline, solemye, amphidesme.

Les corbulées. Corbule, pandore.

(†) Ligament uniquement extérieur.

Les lithophages. Saxicave, pétricole, vénérupe.

Les nymplacées. Sanguinolaire, psammobie, psammotée, telline, tellinide, corbeille, lucine, donace, capse, crassine.

(b) Coquille close aux extrémités latérales, lorsque les valves sont fermées.

Conchifêres lamellipèdes.

Les conques fluviatiles. Cyclade, cyrène, galathée; marines, cyprine, cythérée, vénus, vénéricarde. Les cardiacées. Bucarde, cardite, cypricarde, hyatelle, isocarde.

Les arcacées. Cucullée, arche, pétoncle, nucule. Les trigonées. Trigonie, castalie. 
Les nayades. Mulette, hyrie, anodonte, iridine.

(2) Coquille irrégulière, toujours inéquivalve.

Les camacées. Dicérate, came, éthérie.

\section{Ordre II.}

\section{CONCHIFÈRES MONOMYAIRES.}

Ire Section. - Ligament marginal, allongé sur le bord, sublinéaire.

(a) Coquille transverse, équivalve, à impression musculaire allongée, bordant le limbe supérieur.

Les tridacnées. Tridacne, hippope.

(b) Coquille, soit longitudinale, soit subtransverse, à impression musculaire resserrée dans un espace isolé, sans border le limbe.

(†) Ligament au bord latéral de la coquille, et toujours entier.

Les mytilacées. Modiole, moule, pinne.

(†) Ligament au bord inférieur de la coquille, oa divisé.

Les malléacées. Crénatule, perne, marteau, avicule, pintadine.

$2^{\mathrm{e}}$ Section. - Ligament non marginal, resserré dans un court espace sous les crochets, toujours connu, et ne formant point un tube tendineux sous la coquille.

(a) Ligament intérieur ou demi-intérieur. Coquille régulière, à test compacte, non feuilleté.

Les pectinides. Houlette, lime, plagiostome, peigne, plicatule, spondile, podopside.

(b) Ligament intérieur ou demi-intérieur. Coquille irrégulière, à test feuilleté, quelquefois papyracé.

Les ostracées. Gryphée, huître, vulselle, placune, anomie.

3e Section. - Ligament, soit nul ou inconnu, soit 
DE M. IDE LAMARCK.

représenté par un cordon tendineux qui soutient la coquille.

(a) Ligament et animal inconnu. Coquille très inéquivalve.

Les rudistes. Sphérulite, radiolite, calcéole, birostrite, discine, cranie.

(b) Coquille adhérente, soit immédiatement, soit par un cordon tendineux qui la sontient et lui sert de ligament; l'animal ayant deux bras opposés, ciliés et cirreux.

Les Brachiopodes. Orbicule, térébratule, lingule.

Classe XII.

\title{
LES MOLLUSQUES.
}

\author{
OrdRe I.
}

LES PTÉROPODES.

Hyale, clio, cléodore, limacine, cymbulie, pneumoderme.

ORDRE II.

LES GASTÉROPODES.

i re Section. - Hydrobranches.

(a) Branchies extérieures, placées au-dessus du manteau, soit sur le dos, soit sur ses côtés, et n'étant point dans une cavité particulière.

Les tritoniens. Glauque, éolide, tritonie, scyllée, thétys, doris.

(b) Branchies extérieures, placées sous le rebord du manteau, et disposées en série longitudinale, soit autour du corps, soit d'un seul côté : n'étant pas non pilus dans une cavité particulière.

Les phyllidiens. Phyllidie, oscabrelle, oscabrion, patelle. 
Les semiphyllidiens. Pleurobranche, ombrelle.

(c) Branchies placées dans une cavité particulière sur le dos, située antérieurement près du cou.

Les calyptraciens. Parmophore, émarginule, fissurelle, cabochon, calyptrée, crépidule, ancyle.

(d) Branchies placées dans une cavité particulière, vers la partie postérieure du dos, et recouverte, soit par le manteau, soit par un écusson operculaire.

$(\dagger)$ Point de tentacules.

Les bulléens. Acère, bullée, bulle.

(†) Des tentacules.

Les laplysiens. Laplysie, dolabelle.

$2^{\mathrm{e}}$ Section. - Pneumobranches.

Les limaciens. Onchide, parmacelle, limace, testacelle, vitrine.

OrdRE III.

\section{LES TRACHÉLIPODES.}

I $^{\text {re }}$ Section. - Sans siphon saillant, et respirant en général par un trou. La plupart phytiphages et munis de mâchoires. Coquille à ouverture entière, n'ayant à sa base ni échancrure dorsale subascendante, ni canal.

* Trachélipodes ne respirant que l'air. Coquille spirivalve, mutique, non distinctement nacrée.

(a) Ceux qui habitent hors des eaux.

Les colimacées. Hélix, carocole, anostome, hélicine, maillot, clausilie, bulime, agathine, ambrette, auricule, cyclostome.

(b) Ceux qui vivent dans les eaux, mais qui vienneut respirer l'air à leur surface. Coquille à bords de l'ouverture jamais réfléchie.

Les lymnéens. Planorbe, physe, lymnée.

** Trachélipodes ne respirant que l'eau. Branchieśs saillantes, en forme de filct, de lame ou de houppe, 
DE M. DE LAMARCK.

dans la cavité branchiale. Coquille souvent nacrée, et souvent aussi ayant des parties protubérantes à sa surface.

(a) Coquille fluviatile, operculée, dont le bord gauche n'imite pas une demi-cloison.

(†) Coquille à bords désunis.

Les mélaniens. Mélanie, mélanopside, pirène.

(†) Coquille à bords réunis.

Les péristomiens. Valvée, paludine, ampullaire.

(b) Coquille fluviatile ou marine, dont le bord gauche imite une demi-cloison.

Les néritacées. Navicelle, néritine, nérite, natice.

(c) Coquille marine, dont le bord gauche n'imite pas une demi-cloison.

(†) Coquille flottante à la surface des eaux.

Les janthines. Janthine.

(†) Coquille non flottante, ayant l'ouverture très évasée; point de columelle.

Les macrostomes. Sigaret, stomatelle, stomate, haliotide.

(†十) Ouverture sans évasement particulier; des plis à la columelle.

Les plicacées. Tornatelle, pyramidelle.

(††t) Point de plis à la columelle.

(a) Les bords de l'ouverture réunis circulairement.

Les scalariens. Vermet, scalaire, dauphinule.

(b) Les bords de l'ouverture désunis.

Les turbinacées. Cadran, roulette, troque, monodonte, turbo, planaxe, phasianelle, turritelle.

$2^{\text {e Section. }}$ - A siphon saillant, et ne respirant que l'eau qui parvient aux branchies par ce siphon. Tous sont marins, zoophages, dépourvus de mâchoires, et munis d'une trompe rétractile. Coquille spirivalve, engainante, à ouverture soit canaliculée, soit échancrée ou versante à sa base. 
(a) Coquille ayant un canal plus ou moins long à la base de son ouverture, et dont le bord droit ne change point de forme avec l'âge.

Les canalifères.

Ire Section. - Point de bourrelet constant sur le bord droit.

Cérite, pleurotome, turbinelle, cancellaire, fasciolaire, fuseau, pyrule.

$2^{\mathrm{e}}$ Section. - Un bourrelet constant sur le bord droit.

Struthiolaire, ranelle, rocher, triton.

(b) Coquille ayant un canal à la base de son ouverture, et dont le bord droit change de forme arec l'âge, et a un sinus inférieurement.

Les ailées. Rostellaire, ptérocère, strombe.

(c) Coquille ayant un canal court, ascendant postérieurement, ou une échancrure oblique en demicanal à la base de son ouverture, ce demi-canal se dirigeant vers le dos.

Les purpurifères.

(I) Un canal ascendant, ou recourbé vers le dos.

Cassidaire, casque.

(2) Une échancrure oblique, dirigée en arrière.

Ricinule, pourpre, licorne, concholépas, harpe, tonne, buccin, éburne, vis.

(d) Point de canal à la base de l'ouverture, mais une échancrure subdorsale, et des plis sur la columelle.

Les columellaires. Colombelle, mître, volute, marginelle, volvaire.

(e) Coquille sans canal, mais ayant la base de son ouverture échavcrée ou versante, et ses tours de spire étant larges, comprimés, enroulés de manière que le dernier recouvre presque entièrement les autres.

Les enroulées. Ovule, porcelaine, tarière, ancillaire, olive, cône. 
DE M. DE LAMARGK.

\section{ORDRE IV. \\ LES CÉPHALOPODES.}

jre Division. - Céphalopodes polythalames.

* Coquille multiloculaire, à cloisons simples.

(1) Coquille droite, ou presque droite. Point de spirale. Les orthocérées. Bélemnite, orthocère, nodosaire, hippurite, conilite.

(2) Coquille partiellement en spirale; le dernier tour se continuant en ligue droite.

Les lituolées. Spirule, spiroline, lituole.

(3) Coquille semi-discoïde, à spire excentrique.

Les cristacées. Rénuline, cristellaire, orbiculine.

(4) Coquille globuleuse, sphéroïdale ou ovale; à tours de spire enveloppans ou à loges réunies en tuniques.

Les sphérulées. Miliole, gyrogone, mélonie.

(5) Coquille discoïde, à spire centrale, et à loges rayonnantes du centre à la circonférence.

Les radiolées. Rotalie, lenticuline, placentule.

(6) Coquille discoïde, à spire centrale, et à loges qui ne s'étendent pas du centre jusqu'à la circonférence.

Les nautilacées. Discorbe, sidérolite, polystomelle, vorticiale, nummulite, nautile.

* Coquille multiloculaire, à cloisons découpées sur les bords.

Les ammonées. Ammonite, orbulite, ammonocérate, turrilite, baculite.

$2^{e}$ Division. - Céphalopodes monothalames. Argonaute.

3e Division. - Céphalopodes sépiaires. Poulpe, calmaret, calmar, seiche.

OrdRE V.

LES HÉTÉROPODES.

Carinaire, firole, phyllirhoé. 


\section{MÉTHODE DE M. DE FÉRUSSAC. I8I9.}

DEUXIÈME GRANDE DIVISION DU RÈGNE ANIMAL.

\section{ANIMAUX MOLLUSQUES.}

\section{PREMIĖRE SECTION.}

CÉPIIALÉS.

Classe I.

\section{CÉPHALOPODES.}

I Or ORDRE. LES DÉCAPODES.

re famille. Les ammonées. Genres turrilite, orbulite, ammonite, scaphite, hamite, baculite. $2^{\circ}$ fam. Les hippurites. Batolite, hippurite. 3e fam. Les bélemnites. Bélemnite.

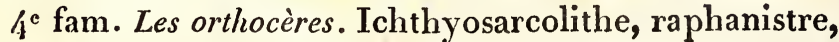
orthocératite, nodosaire.

5. fam. Les lituites. Canope, lituole, spiroline, spirule.

$6^{\mathrm{e}}$ fam. Les discorbes, Cristellaire, discorbe, rotalie. $7^{\mathrm{e}}$ fam. Les nautiles. Lenticuline, nautile.

$8^{\mathrm{e}}$ fam. Les camérines. Sidérolite, nummule, orbiculine, mélonie.

$9^{\circ}$ fam. Les milioles. Rénuline, miliole, globulite, aréthuse.

Io $^{E}$ fam. Les seiches. Seiche, calmar. 
DE M. DE FÉRUSSAC.

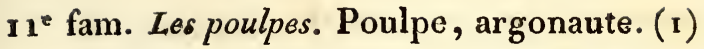

Classe II.

\section{PTÉROPODES.}

$\mathrm{I}^{\mathrm{re}}$ fam. Les hyales. Hyale, cléodore, cymbulie.

$2^{\mathrm{e}}$ fam. Les limacines. Limacine, atlante.

$3^{\mathrm{c}}$ fam. Les clios. Clio.

$4^{\text {e }}$ fam. Les pneumodermes. Pneumodermes, gastéroptère.

5e fam. Les phyllirhoés. Phyllirhoé. (2)

Classe III.

\section{GASTÉROPODES.}

I ER ORDRE NUDIBRANCHES.

ier Sous-Ordre. Anthobranches.

$\mathbf{I}^{\text {re }}$ fam. Les doris. Doris, onchidiore, polycère.

$2^{e}$ Sous-Ordre. Pouxbranchrs.

$2^{\mathrm{e}}$ fam. Les tritonies. Tritonie, doto, thétys, scyllée. 3e fam. Les glauques Laniogère, glauque, éolide, tergipe.

$2^{\ominus}$ ORDRE. INFÉROBRANCHES.

yer Sous-Ordre. Les Peyuluidiens.

$\mathrm{I}^{\text {re }}$ fam. Les phyllidies. Phyllidie, diphyllidie.

(I) Depuis la publication de ses Tableaux systématiques, M. de Férussac, dans un travail commun avec M. Alcide Dorbigny, sur la classe des céphalopodes, a apporté d'importans changemens à sa méthode de classification. (Voyez celle que nous avons adoptée.)

(2) Par suite d'un travail sur la classe des ptéropodes, fait en commun avec M. Rang, la méthode de classification de M. de Férussac sur ces animaux se trouve avoir acquis d'importans changemens. 
$2^{e}$ Sous-Ordre. Les Semi-Phylimiens.

$2^{\mathrm{e}}$ fam. Les ombrelles. Ombrelle.

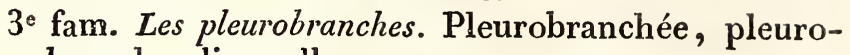
branche, linguelle.

3e Ordre. TEGTIBRANCHES.

$I^{\text {re }}$ fam. Les dicères. Aplysie, actéon, dolabelle, notarche.

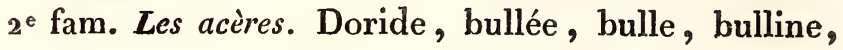
sormet.

$4^{\mathrm{e}}$ Ordre. PULMONÉS SANS OPERCULE.

I er Sous-Ordre. GÉophines.

$\mathrm{I}^{\mathrm{re}}$ fam. Les limaces. Onchide, onchidie, vaginule, philomique, eumèle, véronicelle, limacelle, arion, limace, parmacelle, plectrophore, testacelle.

$2^{*}$ fam. Les limaçons. Hélicarion, hélicolimace, hélice, vertigo, partule.

2e Sous-Ordre. GÉHY DrophinEs.

$3^{\mathrm{e}}$ fam. Les auricules. Carychie, scarabe, auricule, pyramidelle, tornatelle, piétin.

3c Sous-Ordre. Hy grophines.

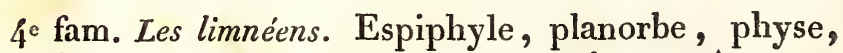
limnée, leptoxe, lomastome, ancyle, eutrême.

5e ORDRE. PULMONÉS OPERCULÉS.

$\mathbf{I}^{\mathrm{re}}$ fam. Les hélicines. Hélicine.

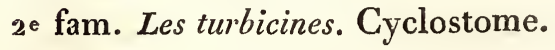

6e Ordre. PECTINIBRANCHES.

y er Sous-Ordre. Les Pomastomes.

$1^{\text {re }}$ fam. Les sabots. Paludine qui se divise en paludine, mélanie, omphémies, risso, littorine ; turritelle, vermet, valvée; natice qui se divise en natice et pitonille. 


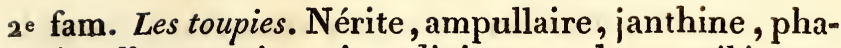
sianelle, toupie qui se divise en sabot, méléagre, monodonte, delphinule, calcar, phorus, cirrhus, solariums, eunphalus, infundibulums, trochus, télescopiums; pleurotomaire, scalaire, mélanopside. $2^{e}$ Sous-Ordre. Hémi-Pomastomes.

Ire Section.

$3^{\mathrm{e}}$ fam. Les cérites. Cérite.

$2^{e}$ Section.

$4^{\circ}$ fam. Les buccins. Buccin qui se divise en buccin et en éburne.

5 e fam. Les pourpres. Pourpre qui se divise en pourpre, nasse, tonne, harpe, casque, cassidaire, struthiolaire et ricinelle; colombelle, rocher qui se divise en rocher, bronte, chicoracé, ranelle et triton; fuseau qui se divise en turbinelle, fasciolaire, pyrule, fuseau, pleurotome, clavatule; rostellaire. $6^{\circ} \mathrm{fam}$. Les strombes. Strombe.

$7^{\mathrm{e}}$ fam. Les cốnes. Cône.

\section{3e Sous-Ordre. Apomastomes.}

8॰ fam. Les enroulés. Olive, ancillaire, marginelle, volvaire, ovule, porcelaine, tarière.

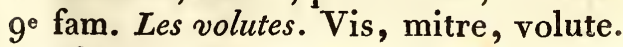
Io fam. Les couronnes. Yet.

$4^{\text {e }}$ Sous-Ordre. AdÉLODERMEs.

I I fam. Les sigarets. Sigaret, cryptostome, lamellaire. $7^{\circ}$ ORDRE. SCUTIBRANCHES.

I er Sous-Ordre.

ire fam. Les ormiers. Haliotide, padolle, stomate. $2^{e}$ Sous-Ordre. Calyptraciens.

$2^{\circ}$ fam. Les cabochons. Cabochon, crépidule. 
$3^{\text {e }}$ fam. Les patelloides. Pavois, flssurelle, émarginule, septaire, calyptrée, trémésie.

3e Sous-Ordre. HÉTÉROPODES.

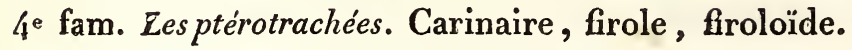
8 OrdRE. CYCLObRaNCHES.

ier Sous-Ordre. Chismobranches.

$\mathbf{I}^{\mathrm{re}}$ fam. Les patelles.

$2^{e}$ Sous-Ordre. Polyplaxiphores.

2' fam. Les oscabrions. Oscabrelle, oscabrion.

DEUXIĖME SECTION.

\section{ACÉPHALÉS.}

\section{Chasse I. \\ CIRRIPÈDES.}

I Or ORDRE. CIRRIPÈDES SESSILES.

$\mathrm{I}^{\mathrm{re}}$ fam. Les balanes. Tubicinelle, coronule, balane, acaste, creusie, pyrgome.

2e OrDRE. CIRRIPÈDES PÉDONCULÉS.

$2^{e}$ fam. Les anatifes. Anatife, pouce-pied, cinéras, brante.

Cuasse II.

\section{BRACHIOPODES.}

$I^{\text {te }}$ fam. Les lingules. Lingule.

$2^{\circ}$ fam. Les terébratules. Térébratule, magas.

$3^{\circ}$ fam. Les cranies. Orbicule, cranie, thécidée. 
DE M. DE FÉRUSAC.

\section{Classe III.}

\section{LAMELLIBRANCHES.}

\section{Ier ORDRE. LES OSTRACÉS.}

Ier Sous-Ordre. Les Monom Xatres.

$\mathbf{I}^{\text {re }}$ fam. Les rudistes. Birostrite, calcéole, radiolite, sphérulite.

$2^{\text {e }}$ fam. Les huitres. Anomie, producte, placune, huître, jodamie, gryphée.

$3^{\text {e }}$ fam. Les pectinides. Podopside, hinnite, spondyle, plicatule, peigne, plagiostome, dianchore, lime, houlette.

$4^{\circ}$ fam. Les malléacés. Marteau, vulselle, perne, inocérame.

$5^{\mathrm{e}}$ fam. Les aviculés. Crenatule, avicule, pintadine, pinne.

$6^{\mathrm{e}}$ fam. Les arcacés. Cucullée, arche, pétoncle, nucule, trigonie.

\section{2e ORDRr. LES MYTILLACÉS.}

$1^{\text {re }}$ fam. Les mytillacés. Moule, modiole, lithodome. $2^{\mathrm{e}}$ fam. Les nayades. Anodonte qui se divise en anodonte, iridine, strophite, lastène, dipsas; hyrie, mulette qui se divise en alasmodonte, amblemides, uniodiés, castalie.

$3^{\mathrm{e}}$ fam. Les cardites. Cardite, cypricarde, vénéricarde. $4^{\mathrm{e}}$ fam. Les crassatelles. Crassine, crassatelle.

3e ORDRE. LES BÉNITIERS.

$\mathbf{I}^{\mathrm{re}}$ fam. Les tridacnés. Tridacne, hippope.

4' ORDRE. LES CARDIACÉS.

$\mathrm{I}^{\mathrm{re}}$ fam. Les camacés. Ethérie, came, dicérate. $2^{\mathrm{e}}$ fam. Les bucardes. Isocarde, bucarde, hémicarde.

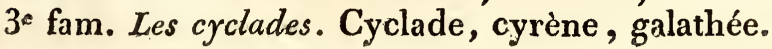


$4^{\circ}$ fam. Les nymphacés. Donace, lucine, loripes, corbeille, tellinide, telline, psammobie, psammotée, donacine, capsa.

5e fam. Les vénus. Cyprine, cythérée, qui se divise en vénus pectinata, vénus scripta, vénus tigerrima, vénus exoleta, vénus.

$6^{\mathrm{e}}$ fam. Les lithophages. Vénérupe, pétricole, corbule, clotho.

$7^{\mathrm{e}}$ fam. Les mactracés. Erycine, mactre, ligule, lavignon, onguline.

\section{ORDRE. LES ENFERMÉS.}

$i^{\text {re }}$ fam. Les myaires. Lutraire, anatine, mye, solémye. $2^{*}$ fam. Les solens. Glycymère, panopée, pandore, solen qui renferme les S.-G. vagina et siliqua; sanguinolaire.

3. fam. Lies pholades. Hyatelle, saxicave, gastrochêne, pholade.

$4^{\mathrm{e}}$ fam. Les tubicolés. Taret, térédine, cloisonnaire, fistulane, clavagelle, arrosoir.

\section{Ciassir IV.}

\section{LES TUNICIERS.}

\section{Or ORDRE. ASCIDIES TÉTHIDES.}

$\mathbf{I}^{\text {re }}$ fam. Les téthyes. Bolténie, cinthie, phallusie, claveline, bipapillaire, mammaire, diazone, distome, sigilline, syncïque, aplide, polyclyne, didemne, eucélie, botryle.

$2^{\mathrm{e}}$ fam. Les lucies. Pyrcsome.

\section{ORdre. ASCIDIES THALIDES.}

fam. Les thalides. Biphore qui comprennent deux S. G., crista nulla et crista dorsali. 


\section{MÉTHODE DE M. DE BLAINVILLE. 1825 .}

\section{TYPE.}

MALACOZOAIRES.

\section{Classe I. CÉPHALOPHORES.}

ORDRE I. CRYPTODIBRANCHES.

Famille I. Octocères. Poulpe.

Fam. 2. Décacères. Calmar, seiche.

Ordre II. CELlulacéS.

Fam. I. Sphérulacés. Miliole, mélonie, saracénaire, textulaire.

Fam. 2. Planulacés. Rénuline, pénérople.

Fam. 3. Nummulacés. Nummulite, hélicite, sidérolite, orbiculine, placentule, vorticiale.

OrdRE III. POLYTHAMACÉS.

Fam. I. Orthocérés. Bélemnite, conulaire, conilite, orthocère, baculite.

Fam 2. Lituacés. Ichthyosarcolithe, lituole, spirule, hamite, ammonocératite.

Fam. 3. Cristacés. Crépiduline, oréade, linthurie.

Fam. 4. Ammonacés. Discorbite, scaphite, ammonite, simplegade.

Fam. 5. Nautilacés. Orbulite, nautile, polystomelle, lenticuline.

Fam. 6. Turbinacés. Cibicide, rotalite. 
Fam. 7. Turriculacés. Turrilite.

\section{Crasse II.}

\section{PARACËPHALOPHORES.}

\section{Sous-Ciasse I.}

\section{PARACEPHALOPHORES DIOIQUES.}

\section{ORDRE I. SIPHONOBRANCHES.}

Fam. I. Siphonostomes. Pleurotome, rostellaire, fuseau, pyrule, fasciolaire, turbinelle, colombelle, triton, ranelle, rocher.

Fam. 2. Entomostomes. Cérite, mélanopside, planaxe alène, vis, éburne, buccin, harpe, tonne, cassidaire, casque, ricinule, cancellaire, pourpre, concholépas.

Fam. 3. Angyostomes. Strombe, cone, tarière, olive, ancillaire, mitre, volute, marginelle, péribole, porcelaine, ovule.

\section{ORDRE II. ASIPHONOBRANCHES.}

Fam. r. Goniostomes. Cadran, toupie.

Fam. 2. Cricostomes. Sabot, pleurotome, dauphinule, turritelle, proto, scalaire, vermet, siliquaire, magile, valvée, cyclostome, paludine.

Fam. 3. Ellipsostomes. Mélanie, rissoaire, phasianelle, ampullares, hélicine, pleurocère.

Fam. 4. Hémicyclostomes. Natice, nérite, navicelle. Fam. 5. Oxystomes. Janthine.

\section{Sous-Crasse II.}

PARACÉPHALOPHORES MONOIQUES.

Section I. Organes de la respiration et corps otectecteur, quand il existe, non symétrique.

OrdRE I. PUlMOBRANCHES.

Fam. I. Limnacés. Limnée, physe, planorbe. 
DE M. DE BLAINVILLE.

Fam. 2. Auriculacés. Piétin, auricule, pyramidelle. Fam. 3. Limacinés. Ambrette, bulime, agathine, clausilie, maillot, tomogère, hélice, vitrine, testacelle, parmacelle, limacelle, limace, onchidie.

ORdRE II. CHISMOBRANCHES.

Coriocelle, sigaret, cryptostome, oxinoé, stomatelle, velutine.

\section{ORDRE III. MONOPLEUROBRANCHES.}

Fam. r. Subaplysiens. Berthelle, pleurobranche, pleurobranchidie.

Fam. 2. Aplysiens. Aplysie, dolabelle, bursatelle, notarche, élysie.

Fam. 3. Patelloïdes. Ombrelle, siphonaire, tilodine. Fam. 4. Acères. Bulle, bellerophe, bullée, lobaire, sormet, gastéroptère, atlas.

Section II. Organes de la respiration et le corps protecteur, quand il existe (ce qui est assez rare), symétriques.

\section{Ordre I. APOROBRANCHES.}

Fam. I. Thécosomes. Hyale, cléodore, cymbulie, pyrgo.

Fam. 2. Gymnosomes. Clio, pneumoderme. Fam. 3. Psilosomes. Phylliroé.

OrdRe II. POLYBRANCHES.

Fam. I. Tétracères. Glaucus, laniogère, tergipède, cavoline, éolide.

Fam. 2. Dicères. Scyllée, tritonie, thétys.

ORDRE III. CYCLOBRANCHES.

Doris, onchidore, péronie.

ORDRE IV. INFÉROBRANCHES.

Phyllidie, linguelle. 
ORDRE V. NUCLÉOBRANCHES.

Fam. I. Nectopodes. Firole, carinaire. Fam. 2. Ptéropodes. Atlante, spiratelle, argonaute.

\section{Sous-Classe III.}

PARACÉPHALOPHORES HERMAPHRODITES.

Section I. Les organes de la respiration et la coquille symétriques.

ORdRE I. CIRRHOBRANGHES.

Dentale.

ORdRE II. GERVICOBRANCHES.

Fam. I. Rétifères. Patelle.

Fam. 2. Branchifères. Fissurelle, émarginule, parmophore.

Section II. Organes de la respiration et coquille non symétriques.

ORDRE III. SCUTIBRANCHES.

Fam. 1. Otidés. Haliotide, ancyle.

Fam. 2. Calyptraciens. Crépidule, calyptrée, cabochon, hipponyce, notrême.

Classe III.

\section{ACÉPHALOPHORES.}

\section{ORDRE I: PALLIOBRANCHES.}

Section I. Coquille symétrique.

Lingule, térébratule, thécidée, strophonème, plagiostome, dianchore, podopside.

Section II. Coquille non symétrique, irrégulière, constamment adliérente.

Orbicule, cranie.

ORDRE II. RUDISTES.

Sphérulite, hippurite, radiolite, birostrite, calcéole. 


\section{ORDRE III. LAMELLIBRANCHES.}

Fan. I. Ostracés. Anomie, placune, harpace, huître, gryphée.

Fam. 2. Subostracés. Spondyle, plicatule, hinnite, peigne, houlette, lime.

Fam. 3. Margaritacés. Vulselle, marteau, pinne, crénatule, inocérame, catile, pulvinite, gervilie, avicule.

Fam. 4. Mytilacés. Moule, jambonneau.

Fam. 5. Pcryodontes ou arcacés. Arche, pétoncle, nucule.

Fam. 6. Submytilacés. Anodonte, mulette, cardite.

Fam. 7. Camacés. Came, dicérate, éthérie, tridacne, isocarde, trigonie.

Fam. 8. Conchacés.

Section r. Bucarde, donace, telline, lucine, cyclade, cyprine, mactre, éricine.

Section 2. Crassatelle, vénus.

Section 3. Vénérupe, coralliophage, clotho, corbule, sphène, onguline.

Fam. 9. Pyloridés.

Section r. Pandore, anatine, thracie, mye, lu tricole.

Section 2. Psammocole, solételline, sanguinolaire, solecurte, solen, solemye, panopée, glycimère, saxicave, byssomie, rhomboïde, hiatelle, gastrochêne, clavagelle, arrosoir.

Fam. 10. Adesmacés. Pholade, térédine, taret, fistulane, cloisonnaire.

ORDRE IV. HÉTÉROBRANCHES.

Fam. 1. Ascidiens.

Tribu x. Ascidiens simples. Ascidie, bipapillaire, fodie.

Tribu 2. Les ascidiens agrégés. Pyure, distome, botrylle, synoïque. 
Fam. 2. Salpiens.

'Tribu 1. Les salpiens simples. Biphore.

Tribu 2. Les salpiens agrégés. Pyrosome.

\section{SOUS-TYPE.}

\section{MALENTOZOAIRES.}

\section{Cinasse I. NÉMATOPODES.}

Fam. ı. Lépadiens. Gymnolèpe, pentalèpe, polylèpe, litholèpe.

Fam. 2. Balanides. Balane, ochthosie, conie, creusie, chtamale, coronule.

Classe II. POLYPLAXIPHORE。

Oscabrion. 


\section{MÉTHODE DF R. IN REILLE. I 825 .}

DEUXIËME SÉRIE DES ANIMAUX.

PREMIÉRE RACE.

\section{LES MOLLUSQUES.}

PREMIÉER BRANCHE.

LES PHA NEROGAMES。

$I^{\text {re }}$ SECTION.

LES PTÉRYGIENS.

$1^{\text {re }}$ CLASSE.

CÉPHALOPODES.

I E ORDRE. DÉCAPODES.

$I^{\text {re }}$ famille. Polythalames.

$\mathrm{I}^{\text {re }}$ tribu. Orthocérates.

Genres bélemnite, callirhoé, ichthyosarcolite, hi bolite, porodrague, acame, cétocine, paclite, pirgopole, téléboïte, achéloïte, chrysaore, hortole, lituite, conilite, nogrobe, hippurite, batolite, tiranite, baculite, hamite, échidne, raphanistre, molosse, réophage, nodosaire, spiroline.

$$
2^{\mathrm{e}} \text { tribu. Polycycliques. }
$$

Spirule, oréade, jésite, charybde, scortime, linthurie, périple, astacole, cancride, pénérople, turrilite, cibicide, cortale, cidarole, storille, ellipsolite, amalté, planulite, ammonie, simplegade.

$3^{\mathrm{e}}$ tribu. Nantilites.

Aganide, plaguse, nautile, angulithe, phonême, elphide, géopone, pélore, chrysole, andromède, 
canthrope, éponide, pharame, sporulie, misile, anténor, robule, spinctérule, clisiphonte, hérione, rhinocure, lampadie, iagrodite, bisiphite, océanie, patrocle, nonione, polyxène, florilis, théméone.

$$
4^{\mathrm{e}} \text { tribu. Milléporites. }
$$

Archidie, ilote, hélénide, cellulie, célibe, borellie, milliolite, clausulie, gyrogonite, rotalite, égéone, tinopore, sidérolite, numulis, licophore, discolite.

$2^{\mathrm{e}}$ fam. Entírostés. Seiche, calmar, sépiole, onikie, cranchie.

$2^{\circ}$ ORDRE. OCTOPODES.

$\mathbb{I}^{\mathrm{re}}$ fam. Acochlides. Poulpe, élédone, léachie.

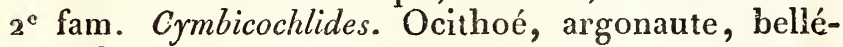
rophe.

\section{$2^{\mathrm{e}}$ CLASSE. \\ PTÉROPODES.}

I ${ }^{2}$ ORDRE. MÉGAPTÉRYGIENS.

$I^{\mathrm{re}}$ fam. Procéphales. Limacine, atlante, clio, cléodore, cymbulie.

$2^{\mathrm{e}}$ fam. Cryptocéphales. Hyale.

$2^{\mathrm{e}}$ ORDRE. MICROPTÉRYGIENS.

$I^{\text {Ie }}$ fam. Pneumodermites. Gastéroptère, pneumaderme.

$2^{\mathrm{e}}$ SECTION.

LES APTÉRYGIENS.

$3^{\text {e CLASSE. }}$

GASTEROPODES.

$I^{\text {re }}$ SECTION. LES HERMAPHRODITES.

I T ORDRE. NUDIBRANCHES.

$I^{\text {re }}$ fam. Urobranches. Carinaire, doris, polycère, onchidiore. 
DE M. LATREILLE。

$2^{\mathrm{e}}$ fam. Séribranches Tritonie, thétys, scyllée.

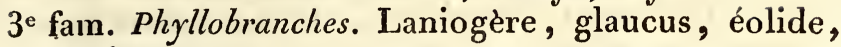
tergipès.

$2^{\mathrm{e}}$ ORDRE. INFÉROBRANCHES.

$\mathbf{I}^{\text {re }}$ fam. Bifaribranches. Phyllidie, diphyllidie, atlas $2^{\text {e }}$ fam. Unabranches. Pleurobranche.

3e ORDRE. TECTIBRANCHES.

$1^{\text {re }}$ fam. Tentaculés. Phillirhoé, notarche, aplysie, actéon, dolabelle, bulline.

$2^{\circ}$ fam. Acérés. Bullée, bulle, sormet, doridie.

$4^{\circ}$ ORDRE. PULMONÉS.

I $^{\text {re }}$ fam. Nudilimaces. Onchide, onchidie, vaginule, véronicelle, limace, arion, limacelle, parmacelle, plectrophore, testacelle.

$2^{\mathrm{e}}$ fam. Géocochlides. Hélicarion, vitrine, ambrette, hélice, caracole, anostome, maillot, grenaille, clausilie, bulime, agathine, vertigo, partule.

$3^{\mathrm{e}}$ fam. Limnocochlides. Carychie, scarabe, auricule, conovule, cassidule, lymnée, physe, planorbe, ancyle.

\section{$2^{\circ}$ SEGTION. LES DIOÏQUES.}

5e ORDRE. PNEUMOPOMES.

$3^{\text {re }}$ fam. Hélicinides. Hélicine.

$2^{\mathrm{e}}$ fam. Turbicines. Cyclostome.

$6^{\circ}$ ORDRE. PECTIN IBRANCHES.

${ }^{3}$ re section. Les Gymnocochlides.

$\mathbf{I}^{\mathrm{re}}$ fam. Péristomiens. Paludine, valvée, vermet, dau= phinule, scalaire.

$2^{\mathrm{e}}$ fam. Turbinés. Turritelle, turbo, ampullaire, janthine.

$3^{\circ}$ fam. Trochoïdes. Troque, cadran, roulette, nomodonte, pleurotomaire.

4. fam. Néritacés. Nérite, natice.

$5^{\circ}$ fam. Mélanides. Phasianelle, mélanie, mélanopside, pyrène, planaxe. 
$6^{\mathrm{e}}$ fam. Plicacés. Tornatelle, pyramidelle.

$7^{\mathrm{e}}$ fam. Fusiformes. Potamide, cérite, cancellaire, fasciolaire, carreau, pleurotome, turbinelle, fuseau, latyre, clavatule, pyrule.

$8^{\mathrm{e}}$ fam. Ailés. Rostellaire, ptérocère, strombe, hippocrène.

$9^{\mathrm{e}}$ fam. Variqueux. Rocher, bronte, typhis, chicoracé, aquille, lotoire, trophone, ranelle, apolle, alectryon, triton, struthiolaire.

$1^{\circ}$ fam. Cassidites. Ricinule, cassidaire, casque. I $^{\mathrm{e}}$ fam. Doliaires. Harpe, tonne, licorne, concholé-

pas, pourpre.

$12^{\mathrm{e}}$ fam. Buccinides. Nasse, buccin, éburne.

I $3^{\mathrm{e}}$ fam. Subulés. Vis.

$14^{\mathrm{e}}$ fam. Columellaires. Mitre, volute, yet, marginelle, colombelle, volvaire.

$15^{\mathrm{e}}$ fam. Conoïdes. Cône.

$16^{\mathrm{e}}$ fam. Olivaires. Olive, tarière, ancillaire.

$17^{\mathrm{e}}$ fam. Ovoïdes. Porcelaine, ovule.

$2^{\circ}$ section. Les Cryptocochlides.

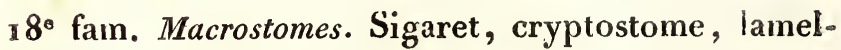
laire.

DEUXIÈME BRANGHE。

\section{LES AGAMES.}

$I^{\text {re. SECTION. }}$

EX OCÉPHALES.

$4^{\circ}$ CLASSE.

PELTOCOCHLIDES

Ier ORDRE. SCUTIBRANCHES.

$\mathrm{I}^{\text {re }}$ fam. Auriformes. Haliotide, stomate, stomatelle.

$2^{\mathrm{e}}$ fam. Piléiformes. Septaire, crépidule, calyptrée, hipponyce, cabochon, émarginule, fissurelle , parmophore. 
$2^{\text {e }}$ ordRe. CYCLOBRANCHES.

$1^{\text {re }}$ fam. Scutiformes. Ombrelle, patelle. $2^{\mathrm{e}}$ fam. Lamellés. Oscabrions, oscabrelle.

$2^{\text {e }}$ SECTION.

\section{ENDOCÉPHALES.}

\section{$5^{\mathrm{e}}$ GLASSE.}

\section{BRACHIOPODES.}

I ER ORDRE. PÉDONCULÉS.

$1^{\text {re }}$ fam. Équivalves. Lingule.

$2^{\mathrm{e}}$ fam. Inéquivalves. Térébratule.

$2^{\mathrm{e}}$ ORDRE. SESSILES.

$I^{\text {re }}$ fam. Fixivalves. Orbicule, cranie, acarde, sphérulite.

\section{$6^{\text {e }}$ cLasse. \\ CONCHIFÈRES}

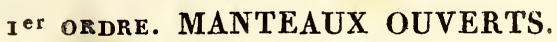

$\mathrm{I}^{\mathrm{re}}$ section. Mésomyones.

$I^{\text {re }}$ fam. Ostracés. Calcéole, gryphée, jodamie, huître, vulselle, producte, podopside, anomie, plicatule, placune, houlette, lime, dianchore, plagiostome.

$2^{\mathrm{e}}$ fam. Pectinides. Peigne, spondyle.

$3^{\text {e }}$ fam. Oxygones. Mullerie, crénatule, gervillie, perne, marteau, pintadine, avicule, pinne.

$2^{\mathrm{e}}$ section. Plagymyones.

伊 fam. Arcacés. Cucullée, arche, pétoncle, nucule, trigonie.

$2^{\mathrm{e}}$ ORdRe. MANTEAUX BIFORÉS.

$1^{\text {re }}$ fam. Mytilacés. Moule, modiole, lithodome.

$2^{\text {e }}$ fam. Nayades. Anodonte, iridine, mulette, hyrie, castalie.

3e ORDRE. MAN'TEAUX TRIFORÉS.

$1^{\text {re }}$ fam. Tridacnites. Hippope, tridacne. 
$4^{\circ}$ ORDRE. MANTEAUX TUBULEUX.

I re section. Uniconque.

$x^{\mathrm{re}}$ fam. Camacés. Éthérie, came, dicérate.

$2^{\circ}$ fam. Cardiacés. Isocarde, cypricarde, bucarde, hémicarde, cardite, vénéricarde.

$3^{\mathrm{e}}$ fam. Cycladines. Cyclade, cyprine, galathée, cyrène.

$4^{\mathrm{e}} \mathrm{fam}$. Vénérides. Cythérée, vénus, vénérupe.

$5^{\mathrm{e}}$ fam. Tellinides. Pétrifore, pétricole, saxicave, corbeille, crassine, lucine, loripède, capse, donace, tellinide, telline, psammobie, psammotée, sanguinolaire.

$6^{\mathrm{e}}$ fam. Corbulés. Corbule, pandore.

$7^{\mathrm{e}}$ fam. Mactracés. Érycine, onguline, crassatelle, mactre, solémye.

$8^{\circ}$ fam. Amplidesmites. Amphidesme.

$9^{\mathrm{e}}$ fam. Myaires. Lutraire, anatine, mye.

${ }_{10}{ }^{\mathrm{e}}$ fam. Solénides. Panopée, hiatelle, glycimère,

solen, gastrochêne, pholadomie, lepton.

${ }_{1} I^{\mathrm{e}}$ fam. Pholadaires. Pholade.

$$
2^{\mathrm{e}} \text { section. Les Tubicoles. }
$$

$12^{ }$fam. T'érédinites. Taret, xylophage, térédine, cloisonnaire, fistulane, clavagelle.

SECONDE RACE.

\section{ELMIN'THOÏDES.}

\section{$I^{\text {re }}$ CLASSE. \\ CIRRIPÈDES.}

I Pr ORDRE. POLYBRANCHES.

$I^{\text {te }}$ fam. Gymnodermes. Cinéras, otion. ${ }_{2}{ }^{\mathrm{e}}$ fam. Ostracodermes. Lithotrye, anatife, pouce-pied.

$2^{\text {e }}$ ORDRE. DIBRANCHES.

$\mathrm{I}^{\text {re }}$ fam. Quadrifores. Tubicinelle, coronule, balane, acaste.

2" fam. Bifores. Creusie, pyrgome. 



\section{TABLEAU SYNOPTIQUE}

\section{DE LA CLASSIFICATION}

SUIVE DANS LE MANUEL DE L'HISTOIRE NATURELLE DES MOLLUSQUES ET DE LEURS COQUILLES.

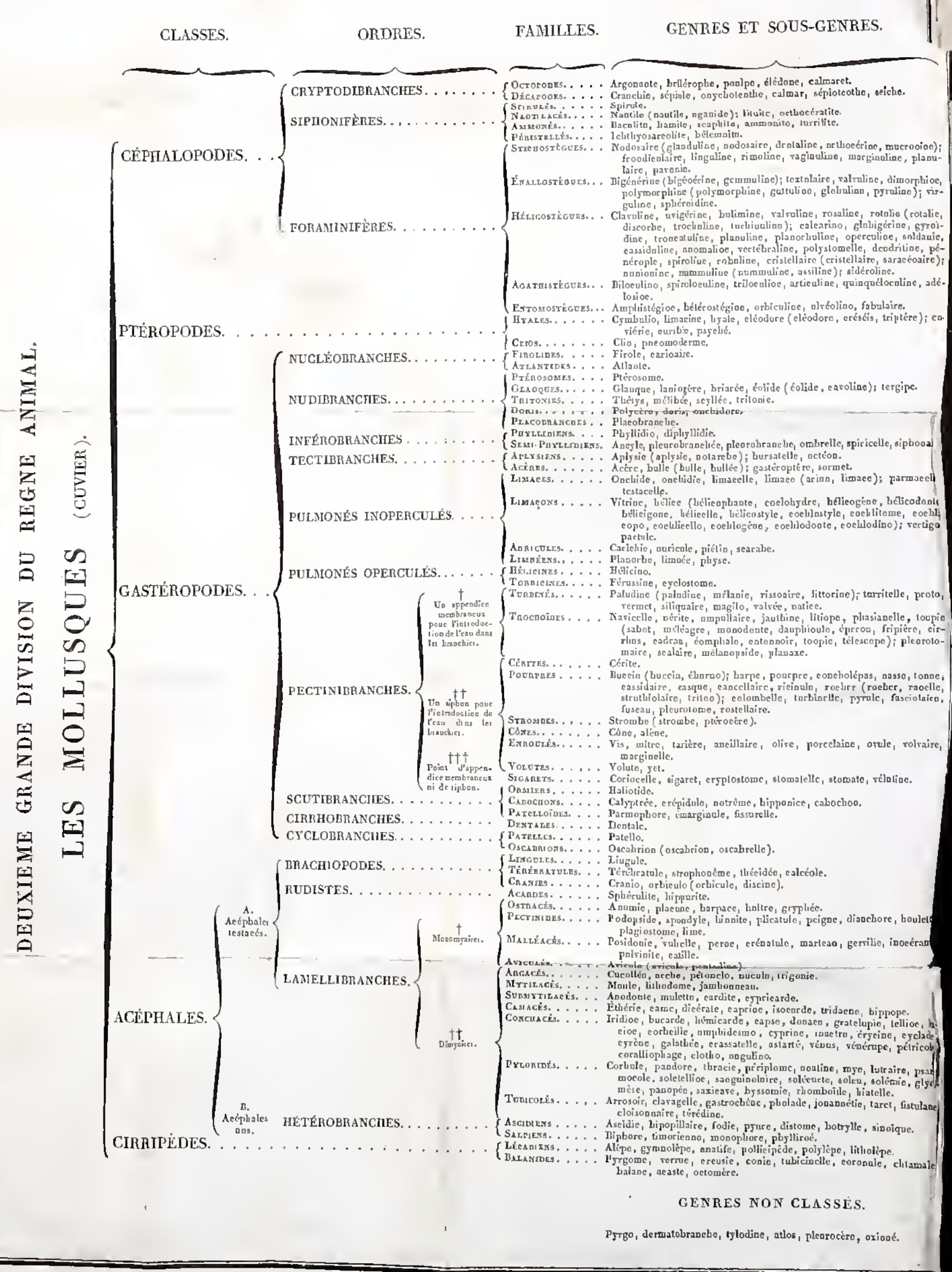




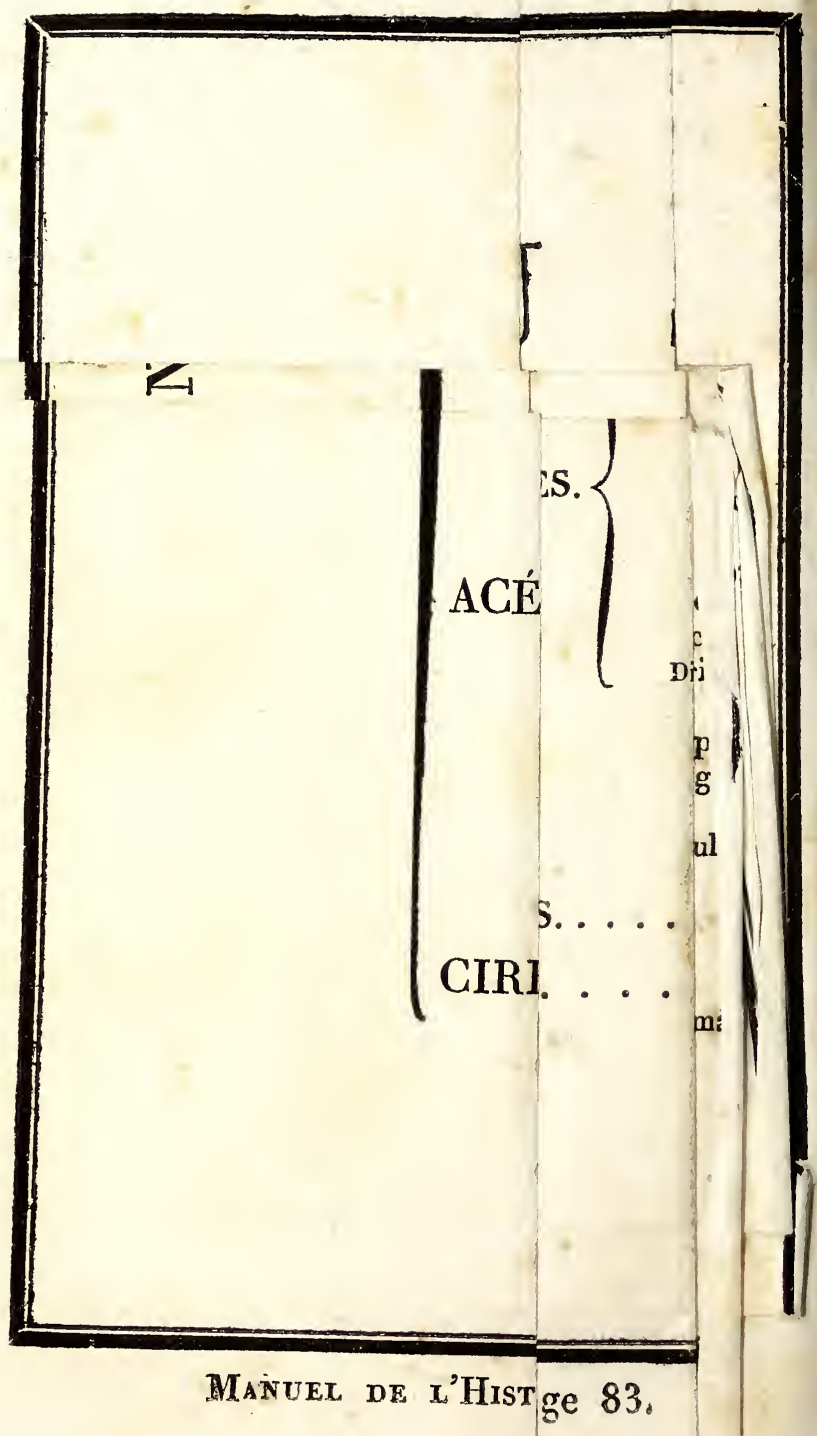




\section{TABLEAU}

\section{MÉTHODIQUE ET CARACTÉRISTIQUE}

DE TOUS LES GENRES

\section{DE MOLLUSQUES.}

PREMIÉRE CLASSE.

\section{CÉPHALOPODES, Cuvier.}

Cryptodibranches, Blainv., prem. classif.; Céphalophores, Blainv., malac.; Antlio-brachiophora, Gray.

Animal renfermé dans une tunique musculeuse, ouverte par-devant, et d'où sort une tête très distincte; bouche antérieure et terminale, armée de deux mandibules cornées, très fortes, agissant verticalement l'une sur l'autre, entre lesquelles se trouve une langue garnie de pointes cornées; yeux sessiles, généralement très grands; organes locomoteurs composés d'appendices tentaculiformes, ou bras, au nombre de huit, dix ou plus, entourant la bouche, servant à la préhension, et quelquefois à la natation; organes de la respiration, branchiaux, pairs et symétriques, cachés dans une poche dorsale; anus médian, caché à la partie antérieure du sac; sexes séparés sur deux individus différens. Coquille univalve, inoperculée, rudimentaire, monothalame ou polythalame et très variée dans sa forme; extérieure en tout ou en partie, ou tout-à-fait interne ; munie, lorsqu'elle est cloisonnée, d'un siphon continu ou de petites ouvertures à chaque cloison.

Tous marins, ordinairement libres, rarement fixés. 
I $^{\text {er }}$ ORDRE.

\section{CRYPTODIBRANCHES, Blainville.}

Céphal. testacés monothalames et non testacés (ou sépiaires ), Lam.; Octopodes et fam. des Seiches, Fér., Tab. méth.; Octopodes et fam. des Entérostés, Lat.

Anim. en forme de sac ou de bourse, avec ou sans nageoires; tête grosse, bien distincte, couronnée d'appendices brachiaux au nombre de huit ou dix; bras quelquefois très inégaux, toujours garnis de ventouses à leur face interne, avec ou sans crochets; branchies pyramidales; oritice des organes de la génération s'ouvrant dans la poche branchiale, celle-ci communiquant à l'extérieur par une sorte d'entonnoir, dont le tube s'ouvre sous le cou.

Coq. monothalame ou rudimentaire, alors cornée ou celluleuse, mais jamais polythalame.

$$
I^{\text {re }} \text { FAMILLE. }
$$

\section{LES OCTOPODES, Leach.}

Sépiaires, Lam.; Octocères, Blainv.; Acochlides et Cymbicochlides, Lat.

Anim. raccourci, bursiforme, ordinairement dépourvu de nageoires; tête bien distincte; huit bras sessiles, très longs, communément presque égaux, se repliant dans le test, lorsqu'il existe, et munis de ventouses simples; nu ou testacé, point de rudiment testacé interne, mais seulement deux petites pièces cartilagineuses dans quelques espèces. Coq. monothalame en cône très oblique et à sommet spiral.

† Une coquille extérieure renfermant tout l'animal.

Genre I. Argonaute, argonauta, Linné. Et Ocythoé, Rafin.

Anim. ovoïde ou elliptique, entièrement renfermé 
dans une coquille uniloculaire, sans attache musculaire; bras libres à leur base, presque égaux, se repliant dans le test dans l'état de repos; garnis sur leur base interne de ventouses pédiculées, en forme de cupule, et alternant sur deux séries; deux d'entre eux, les bras intermédiaires supérieurs, élargis vers leur extrémité en forme d'ailes ou de voiles.

Coq. uniloculaire, papyracée, mince, fragile et transparente, en cône oblique, à sommet plus ou moins spiral, aplatie sur les côtés, à carène large ou étroite; spire courte et rentrant dans l'ouverture.

Ce genre, l'un des plus anciennement connus et des plus répandus dans la mer, donne cependant encore lieu à une grande divergence d'opinion parmi quelques naturalistes, plusieurs d'entre eux reconnaissant dans le mollusque Céphalopode que l'on trouve toujours dans cette coquille, et que nous venons de décrire, son véritable auteur, tandis que d'autres persistent à ne voir en lui qu'uu animal parasite qui viendrait s'y loger après en avoir chassé ou dévoré le véritable propriétaire. Ces deux opinions sont appuyées de part et d'autre sur de savantes objections; cependant cet état d'incertitude ne saurait durer aujourd'hui, que nous possédons les beaux travaux de Poli sur ce curieux animal. Les Argonautes sont des mollusques pélagiens que l'on ne trouve que dans les mers chaudes et une partie de celles des zones tempérées; on les rencontre pendant les calmes et quelquefois pendant les orages; I' $A$. argo, qui est commun dans la Méditerranée, est souveut jeté sur nos côtes du midi. Il y en a encore quatre ou cinq espèces. Les Ocythoés ne diffèrent en rien des Argonautes.

G. II. Bellérophe, bellerophon, Montfort. Anim inconnu.

Coq. uniloculaire, mince, plus ou moins spirale, globuleuse ou un peu déprimée par le dos, et alors en forme de navette; spire visible, ou cachée quelquefois par le dernier tour qui enveloppe les autres; 
bouche arquée, recevant dans son milieu l'avantdernier tour, et prolongée aux deux extrémités; une carène dorsale formant une sorte de sinus au bord dorsal de l'ouverture.

Ce genre, seulement connu à l'état fossile, et qui, d'après de nouvelles observations de M. Defrance, ne serait point cloisonné, et alors n'appartiendrait point aux Céphalopodes, ne renferme que quelques espèces la plupart venant d'Angleterre; M. de Férussac les divise en trois groupes.

I $^{\text {er }}$ groupe. Ovuliformes.

Tuberculatus, etc.

$2^{\mathrm{e}}$ groupe. Nautiloïdes.

Costatus, etc.

Apertus, etc.

$3^{\mathrm{e}}$ groupe. Helicoïdes.

$\dagger \dagger$ Point de coquille extérieure.

a) Sac court, dépourvu de nagecires.

r) Deux rangées de ventouses alternant le long de chaque bras.

G. III. Poulpe, octopus, Lamarck.

Anim. muni d'un sac plus ou moins globuleux, obtus à son extrémité inférieure, épais, très musculeux, sans appendices natatoires et à ouverture étroite; bras communément réunis à leur base par une large membrane.

Coq. aucune; deux petites pièces cartilagineuses internes.

Les Poulpes habitent dans toutes les mers, et nous en possédons aussi sur nos côtes : mais ils sont peu connus, quoique cependant très à notre portée; nous avons rencontré au milieu de l'Océan une espèce bien distincte des autres, d'une couleur rouge très foncée, ayant les bras courts, et de la grosseur d'un tonneau. Cette observation nous donne lieu de penser que Montfort n'a peut-être fait qu'exagérer un peu ce qu'il a dit des Ponlpes gigantesques, et Kraken. La science doit à M. Cuvier un beau travail ana- 
tomique sur ce geure, et MI. de Férussac en a déjà reconnu un bọ nombre d'espèces.

2) Une seule rangée de ventouses le long de chaque bras.

$$
\begin{aligned}
& \text { G. IV. ÉlÉdone, eledon, Leach. } \\
& \text { Ozoema, Raf. }
\end{aligned}
$$

Anim. semblable au Poulpe, mais s'en distinguant par une seule rangée de ventouses à chaque bras.

Ce genre ne renferme qu'un très petit nombre d'espèces.

b) Sac plus allongé, pourvu de nageoires unies ou séparées; bras sessiles presque égaux; ventouses?

\section{G. V. Calmaret, loligopsis, Lam. Leachia, Lesueur.}

Anim. muni d'un sac oblong, pointu à son extrémité, avec une nageoire circulaire qui embrasse sa partie postérieure, ou des nageoires latérales triangulaires et terminales.

M. de Férussac pense, d'après le peu de connaissances que l'on a de ce genre, qu'il devra ’eut-être se réunir aux Cranchies.

\section{$2^{2}$ FAMille.}

\section{LES DÉCAPODES, Leach.}

Sépiolés ou Sépiaires, Lam.; Décacères, Blainv.; Entérostrés, Lat.

Anim. généralement allongé, cylindriforme, ayant un sac épais, muni d'expansions natatoires; tête bien distincte, couronnée par dix bras, dont huit sont sessiles et sétacés, plus courts que dans la famille précédente, et garnis de ventouses tout le long de leur face interne, et deux beaucoup plus longs, rétractiles, pédiculés, hors de rang, garnis de ventouses ordinairement sur la portion élargie et terminale seulement.

Coq. aucune, seulement un rudiment testacé, cartilagineux ou calcaire dans l'intérieur du dos, dans. la plupart des genres. 
$†$ Nageoires partielles, postérieures ou subdorsales.

G. VI. Cranchie, cranchia, Leach.

Anim. muni d'un sac bursiforme, allongé, arrondi postérieurement; ouverture étroite, bord dorsal non distinct, retenu par des brides; tête moins distincte du corps; bras sessiles, inégaux ; bras pédonculés, plus longs, rétractiles, terminés en massue; pédoncules dépourvus de ventouses; nageoires caudales, circulaires, se touchant à leur origine. Rudiment interne inconnu.

Nous ne possédons que peu de connaissances sur ce genre dont on ne cite que trois espèces. D'après $M$. de Blainville, l'une d'elles, la $C$. cardioptera, aurait une lame interne comme les Calmars.

\section{G. VII. SÉPIOLE, sepiola, Leach.}

Anim. sacciforme, raccourci, déprimé, arrondi postérieurement; bras sessiles, presque égaux; bras pédonculés, terminés en massue; point de ventouses sur les pédoncules.

Rudim. interne, très grêle et très petit.

On n'en connait qu'une seule espèce qui est de la Méditerranée.

G. VIII. Onyснотеuтнг, onychoteuthis, Lichtenst. Onychia, Les.

Anim. muni d'un sac cylindracé, acuminé postérieurement, et à bord dorsal bien distinct du cou; nageoires grandes, formant un rhombe par leur réunion; bras sessiles, assez égaux, quelquefois armés de griffes; bras pédonculés, longs, terminés en massue, et armés de ventouses et de griffes cornées et inégales.

Rudim. interne, corné, étroit, en forme d'épée à trois tranchans.

Onze espèces, toutes exotiques, composent ce genre; mais il paraît que quelques unes d'elles seraient des variétés. d'âge. 


\section{G. IX. Calmar, loligo, Lam.}

Anim. muni d'un sac allongé, cylindracé, acuminé postérieurement; bord dorsal du sac bien distinct du cou, quelquefois prolongé en pointe; nageoires comme dans le genre précédent ; bras sessiles, assez égaux; bras pédonculés, longs et terminés en massue; les ventouses garnies quelquefois de dents ou de crochets, dans une portion de leur circonférence, mais jamais de véritables griffes.

Rudim. interne, corné, mince, transparent, quelquefois partiellement gélatineux, de forme un peu variable, mais en général élargi et aplati en forme de plume.

Les Calmars, déjà assez nombreux en espèces, sont divisés en deux groupes par M. de Férussac.

$$
\text { I er groupe. }
$$

Les pédoncules des bras munis de ventouses sur presque toute leur longueur.

$L$. sagittata, etc.

$$
2^{\mathrm{e}} \text { groupe. }
$$

Les pédoncules des bras longs dépourvus de ventouses.

L. bartramii, etc.

Ce dernier groupe est le plus nombreux.

†† Nageoires latérales, s'étendant tout le long du sac.

G. X. Ś́рготитнг, sepiotheuthis, Blainv.

Anim. allongé, bordé dans presque toute sa longueur par une membrane natatoire, étroite et latérale; bras sessiles et pédonculés, comme dans les Calmars, moins élargis que dans les Seiches; ventouses également comme dans les Calmars.

Rudim. interne, corné et mince.

\section{G. XI. Sеiche, sepia, Lam.}

Anim. ovale, déprimé, bordé dans toute sa longueur 
par une membrane natatoire, étroite et latérale; tête très grosse; bras sessiles, courts et triangulaires; bras pédonculés, longs, et terminés en massue; ventouses à bords cornés, non dentés.

Rudim. interne, calcaire, ovale, épais, lamelleux, celluleux, bombé en dessus et en dessous, terminé postérieurement par une pointe médiane plus dure.

Ce genre ne renferme qu'un petit nombre d'espèces, la plupart des mers de l'Inde; nous en possédons cependant une sur nos côtes, $S$. officinalis, qui fournit un aliment abondaut à la classe du peuple.

$$
\text { I er groupe. }
$$

Plusieurs séries de ventouses.

S. tuberculata, etc.

$$
2^{\mathrm{e}} \text { groupe. }
$$

Deux sérics seulement de ventouses sur les bras. S. affinis, etc.

On doit à M. Cuvier la connaissance des débris fossiles d'os de Seiche qui avaient donné lieu à l'établissement du genre Beloptère de M. Deshayes, adopté par M. de Blainville. M. Dorbigny a décrit ces fossiles sous les noms de S. Cuvieri et parisiensis.

\section{$2^{\mathrm{e}}$ ORDRE.}

\section{SIPHONIFERE, Dorbigny.}

Céphalopodes testacés polythalames, Lam.; Décapodes. F'ér. Lat.

Anim. peu connu, ayant dix bras, ou plus, entourant la bouche.

Coq. polythalame, externe, ou partiellement recouverte par l'animal, qui peut alors rentrer en tout ou en partie dans une loge supérieure à la dernière cloison; un siphon toujours continu d'une loge à l'autre. 
I re FAMILLE.

\section{LES SPIRULÉS, Dorb.}

Anim. muni de huit bras sessiles et de deux bras pédonculés, entourant la bouche, et garnis de ventouses, les deux derniers se repliant dans le sac. Coq. simple, spirale; cavité supérieure à la dernière cloison, presque nulle; cloisons unies, siphon au bord intérieur.

\section{G. XII. Sprrure, spirula, Lam.}

Anim. bursiforme, entourant partiellement une coquille dans sa partie postérieure; tête munie de dix bras, dont deux contractiles et pédonculés. Coq. contournée en spirale, discoïde, à tours distans les uns des autres; siphon au bord interne.

L'animal de la Spirule est très incomplétement connu malgré les différentes figures que l'on en a faites, et il paraît que celle qui mérite le plus de confiance a été donnée par M. de Lamarck dans l'Encyclopédie méthodique. On ne connaît encore qu'une seulc espèce, la $S$. Peronii, très commune dans les collections, et que nous avons souvent rencontrée en pleine mer, flottant avec quelques faibles débris de l'animal. On doit à M. Dorbigny d'avoir reconnu les deux bras pédonculés et contractiles.

$2^{\text {e FAMILLE, }}$

\section{LES NAUTilaCÉS, Dorb.}

Anim. muni d'une série très nombreuse de bras sessiles, dans les animaux connus, et entourant la bouche; ventouses?

Coq. simple, spirale, ou droite; cloisons unies; cavité supérieure à la dernière cloison, grande et engaînante; siphon toujours central ou situé au bord intérieur.

† Test spiral. 


\section{G. XIII. Nautile, nautilus, Linné.}

Et Aganide, Angulite, Canthrope, Oceanie, Bisiphite, Ammonie, Montf.

Anim. bursiforme, logé en partie dans la dernière loge de la coquille, muni postérieurement d'un appendice ou organe particulier, destiné à traverser toutes les loges, et placé dans un siphon qui lui sert de gaîne; manteau se prolongeant au-dessus de la tête, qui est munie d'un grand nombre de bras sessiles entourant la bouche.

Coq. discoïde, en spirale régulière, roulée sur le même plan, embrassante ou non, à tours contigus; siphon central ou placé contre le retour de la spire.

I'animal de ce genre, qui renferme beaucoup de coquilles fossiles, n'est connu que par la figure de Rumphius qui laisse tant à désirer. M. Dorbigny réunit aux Nautiles plusieurs genres de Montfort qui, en effet, ne semblent pas devoir en être sèparés; il établit en même temps les sousgenres suivans.

$1^{\text {er }}$ Sous-Genre. Nautiles proprement dits.

Anim. comme dans la caractéristique du genre.

Coq. à spire embrassante ou non; cloisons unies; siphon central ou rapproché de l'avant-dernier tour de spire, sans lui être contigu.

$$
\mathrm{I}^{\mathrm{er}} \text { groupe. }
$$

Spire embrassante. (G. Nautile, Angulite, Océanie $e_{\text {\%.n }}$ Montf.)

N. Pompilius, etc.

$$
2^{\mathrm{e}} \text { groupe. }
$$

Spire à découvert. (Ammonie, Montf.)

$N$. umbilicatus, etc.

$2^{\mathrm{e}}$ S.-G. Aganides, Montf.

Anim. inconnu.

Coq. à spire embrassante; cloison désunie par deux prolongemens intérieurs de ses bords; siphon touchant l'avant-dernier tour de spire. 


\section{G. XIV. Liturte, lituitus, Montf. \\ Et Hortole.}

Anim. inconnu.

Coq. spirale, à spire projetée en ligne droite, à un certain âge, de manière que la volute figure une sorte de crosse; tours détachés ou contigus ; siphon contre le retuur de la spire; dernière loge très longue et engaînante.

Toutes les espèces de ce genre sont fossiles.

†† Test non spiral.

G. XV. Orthocératite, orthoceratites, Montf.

Anim. inconnu.

Coq. non spirale, conique, projetée en ligne droite ou arquée; siphon central ou marginal; dernière loge très grande et engaînante.

Un siphon central.

I ${ }^{\mathrm{er}}$ groupe.

O. striata, etc.

$2^{e}$ groupe.

Un siphon marginal.

$O$. undulata, etc.

Toutes les espèces de ce genres sont fossiles.

3e Famille.

LES AMMONÉES, Lam.

Anim. inconnu.

Coq. spirale ou droite, simple ; cloison découpée ; cavité supérieure à la dernière cloison, très grande et engaînante; siphon marginal.

Cette famille ne renferme que des coquilles fossiles.

† Coquille droite; point de spire.

G. XVI. Baculite, baculites, Lam. Homolocératite, Hubsch; cératoides, Schunch.; tirannitc, Montf.; rhabdites, Haan.

Anim. inconnu. 
Coq. droite, cylindrique, quelquefois un peu déprimée, conique; cloisons sinueuses, peu distantes, la dernière très grande et engaînante; siphon marginal.

Ce genre ne contient qu'un très petit nombre d'espèces. $\dagger \dagger$ Coquille arquée ou spirale et alors projetée en ligne droite à un certain âge.

G. XVII. Hamite, hamites, Sowesby. Ammonocérate, Lam.; Toxerite, Rafin.?

Anim. inconnu.

Coq. arquée, courbée en siphon, ou spire projetée en ligne droite, à un certain âge; cloisons sinueuses, la dernière loge engainante, siphon marginal.

G. XVIII. Scapuite, scaphites, Sow.

Anim. inconnu.

Coq. elliptique, à spire embrassante, roulée sur le même plan; tours contigus, excepté le deraier, qui se détache et se replie ensuite sur la spire; bouche toujours munie d'un bourrelet circulaire dans les individus complets.

G. XIX. Ammonite, ammonites, Bruguière. Et Orbulite, Planorbite, Planulite, Lam.; Ellipsolite, Amalté, Pélaguse, Simplegade, Montf.; Ammonelliptites, Park.; Ophiopomorphites, Plott.; Globites, Ceratites et Goniatites, Haan.

Anim. inconnu.

Coq. discoïde, à spire enroulée sur le même plan, enveloppante ou non; tours contigus, cloisons plus ou moins lobées sur leurs bords par des ramifications ou des digitalions; dernière loge tenant tout le dernier tour de spire.

M. Defrance et M. Dorbigny ont remarqué une disposition particulière dans la forme des bords de la bouche des Ammonites; quelquefois ces bords sont munis d'un bourrelet épais et réfléchi en dehors; d'autres fois deux 
languettes allongées en pointe ou digitées s'étendent de chacun de ses côtés; enfin dans quelques autres, ajoute M. Dorbigny, un troisième appendice part du milieu des deux languettes, et se replie sur l'entrée de la bouche.

$$
\text { I er groupe. }
$$

Spire embrassante. A. subradiatus, etc.

$$
2^{\mathrm{e}} \text { groupe. }
$$

Spire demi-enveloppante.

A. Mantelii, etc.

$$
3^{\mathrm{e}} \text { groupe. }
$$

Spire tout-à-fait à découvert.

A. gigantius, etc.

Le genre Ammonite renferme un grand nombre d'espèces; quelques unes d'elles sont d'une très grande dimension.

G. XX. Turrilite, turrilites, Lam.

Anim. inconnu.

Coq. en spirale turriculée, à tours contigus et tous apparens; dernière loge très grande, formant un tour et demi de spire; siphon marginal.

Un petit nombre d'espèces forme ce genre très distinct des précédens.

$$
4^{\text {e FAMIILE. }}
$$

\section{LES PÉRISTELLÉES, Dorb.}

Anim. inconnu.

Coq. présumée tout-à-fait interne, composée d'un noyau divisé en loges et d'une enveloppe souvent épaisse, formée par un réseau qui a quelque analogie avec celui du rudiment testacé des Seiches; cloisons engaînantes, siphon communément marginal.

Toutes les coquilles de cette famille ne sont encore consues qu'à l'état fossile.

† Coquille spirale. 
G. XXI. Iснтнyosancolite, ichthyosarcolites, Desmarest. Rhabdites, Haan.

Anim. inconnu.

Coq. discoïde, à spire régulière, enroulée sur le même plan, non enveloppante; tours non contigus, séparés les uns des autres par un réseau composé de petits cylindres parallèles suivant les tours du siphon ; dernière loge très grande et engaînante.

Ce genre, qui ne renferme qu'une seule espèce, est très incomplétement connu.

$\dagger †$ Coquille droite.

G. XXII. Bélemnite, Belemnites, Cuv.

Cétocine, Acarne, Ammimone, Chrysaore, Achéloïte, Hibolite, Porodrague, Pyrgopole, Thalamule, Callirhoé, Paclite, Montf.

Anim. inconnu.

Coq. droite, en cône allongé, plus ou moins déprimé, acuminé par un bout et ouvert de l'autre, composé de deux parties distinctes, d'un entourage formé par un réseau de petites locules serrées, transversales et divergentes du centre à la circonférence, enveloppant une série de loges transversales qui forment un ensemble conique, percé le plus souvent, du côté marginal, d'un siphon continu.

Ce genre est très nombreux en espèces. M. Dorbigny les divise en deux groupes.

$$
1^{\mathrm{er}} \text { groupe. }
$$

Coquilles à gouttières latérales.

$B$. digitus, etc.

$$
2^{\mathrm{e}} \text { groupe. }
$$

Coq. sans gouttières.

B. deus, etc.

On doit à M. de Blainville une monographie des Bélemnites, et $M$. Raspail vient tout récemment de faire connaître dans un Mémoire sur ce genre quelques espèces nouvelles 
de la Provence. Ce naturaliste pense que les Bélemnites ne sont point des débris d'animaux mollusques, comme on le croit généralement; mais plutôt des appendices cutanés d'un animal marin, peut-être voisin des Echinodermes; et il dit que l'alvéole est un être étranger à la bélemnite, et qu'il désigne sous le nom d'aivéolite. L'opinion la plus générale. ment adoptée est celle qui les place dans les Céphalopodes.

\section{$3^{\text {e }}$ ORDRE.}

\section{FORAMINIFÈRES, Dorb. (I)}

\section{Asiphonoides, Haan.}

Anim. bursiforme, contenant la coquille dans sa partie postérieure, quelquefois très grand, comparativement au volume de la tête; celle-ci très petite, peu ou point distincte du corps, se renfermant quelquefois presque en entier dans les replis antérieurs de la peau, et terminée par des tentacules nombreux formant plusieurs rangées autour de la bouche qui est centrale.

Coq. polythalame, totalement interne; dernière cloison terminale, point de siphon, mais seulement une ou plusieurs ouvertures donnant communication d'une loge à l'autre.

C'est particulièrement sur les animaux qui forment cet ordre, que l'on doit à $M$. Dorbigny de précieuses découvertes dans la classe des Céphalopodes; de longues et minutieuses recherches sur les coquilles microscopiques l'ont mis à même de nous dévoiler la connaissance d'un grand nombre de genres riches en espèces dont il a reproduit en relief et sur une plus grande échelle des modèles en plâtre. Par le moyen de cet ingénieux travail que les savans de tous les pays se sont empressés d'acquérir, on peut mieux juger de l'organisation singulière de ces petites coquilles, que leur petitesse avait jusqu'ici en grande partie écartées des travaux des naturalistes.

(r) La terminaison en ite des noms de genres indiquera ceux qui ne comprennent que des coquilles fossiles, les autres eu renfermant de vivantes et de fossiles. 
C'est dans cet ordre, nous apprend MI. Dorbigny, que l'on peut trouver des Céphalopodes fixés par quelques parties de l'animal ou par la coquille elle-même, et il ajonte que cette privation de locomotion peut faire présumer chez ces mollusques la réunion des deux sexes sur le même individu.

"Ces animaux, dit-il, vivent sur les côtes, dans les en“ droits peu profonds, et paraissent préférer pour leur a nourriture, telle ou telle espèce de polypes dont ils sont " très friands; il en existe des myriades sur tous les bords “ de la mer; les côtes de l'Océan européen sont peu riches “ en espèces, et elles y sont très petites. Les bords de la “ mer Adriatique paraissent être les plus favorisés sous ce " rapport; on y trouve des genres et des espèces variés et “ d'une taille plus grande. Les terrains tertiaires surtout “ fourmillent en espèces fossiles; quelques unes s'y trouvent “ en si grande abondance, qu'elles peuvent même caracté“ riser des couches entières; les terrains plus anciens n'en " sont pas entièrement dépourvus; la craie de Meudon " fournit des espèces bien caractérisées, ainsi que le cal" caire de Caen, celui des bords de la Gironde, et le cal" caire jurassique du département de la Charente-Infé" rieure, où la conservation des coquilles en nature paraît " être une chose surprenante. »

\section{$l^{\text {re }}$ FAMTLLE.}

\section{LES STICHOSTÉGUES, Dorb.}

Anim. Voyez la caractéristique de l'ordre.

Coq. ayant les loges empilées ou superposées sur un seul axe, bout à bout, soit qu'elles débordent ou non en se recouvrant plus ou moins latéralement; point de spirale.

† Une seule ourerture, centrale.

\section{G. XXIII. Nodosarre, nodosaria, Lam. Orthocère, Lam.; Réophage, Montf.}

Coq. Loges généralement globuleuses, superposées sur un axe fictif, communément droit et central, au bout duquel est l'ouverture terminale, variant dans leurs rapports, depuis l'enchassement partiel, jusqu'à la séparation par érranglement. 
Ce genre se divise en plusieurs sous-genres.

\section{$1^{\text {ex }}$ S.-G. Glandulines, Dorb.}

Coq. Loges globuleuses, partiellement enchàssées les unes dans les autres, et formant par leur réunion un ovoïde; axe central et droit, ouverture au bout d'un prolongement de la dernière loge et terminale.

\section{$2^{\circ}$ S.-G. Nodosaires proprement dites.}

Coq. Loges globuleuses, distinctes, empilées les unes au bout des autres, quelquefois séparées jusqu'à l'étranglement, formant dans leur ensemble un petit cylindre; axe fictif droit et central, ouverture au bout d'un prolongement terminal.

Ce sous-genre, très nombreux en espèces vivantes et fossiles, se divise en deux groupes.

$$
{ }^{\mathrm{er}} \text { groupe. }
$$

Coq. non striées longitudiualement.

N. radicula, etc.

$$
2^{\mathrm{e}} \text { groupe. }
$$

Coq. striées longitudinalement.

N. cequalis, etc.

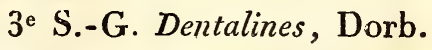

Coq. Loges globulenses ou déprimées, plus ou moins distinctes, quelquefois très obliques, composant un petit cylindre; axe fictif, toujours arqué; ouverture souvent submarginale, avec ou sans prolongement terminal.

Ce sous-genre, assez nombreux, comprend aussi deux groupes.

$$
\text { I er groupe. }
$$

Coq. sans stries longitudinales.

N. communis, etc.

$$
2^{\mathrm{e}} \text { groupe. }
$$

Coq. striées longitudinalement.

N. depressa, etc. 
100

fe S.-G. Orthocérines, Dorb.

Coq. Loges cylindrico-coniques, superposées sans étranglement; test conique et droit, point de prolongement terminal pour l'ouverture.

5e S.-G. Mucronines, Dorb.

Coq. Loges déprimées, superposées, enchâssantes, formant un test conico-cylindrique avec deux lames latérales; un prolongement terminal.

G. XXIV. Frondrculaire, frondicularia, Defrance. Rénuline, Blainv.

Coq. Loges très déprimées sur les deux faces, plus ou moins enchâssantes de chaque côté de l'axe fictif, qui est droit et central.

G. XXV. Lringuline, lingulina, Dorb.

Coq. Ouverture en fente et terminale, loges recouvrantes, test déprimé sur ses faces.

†† Ouverture marginale.

G. XXVI. Rrmuline, rimulina, Dorb.

Coq. Ouverture formant une fente longitudinale, test en forme de gousse à loges obliques et embrassantes.

G. XXVII. Vaginuline, vaginulina, Dorb.

Coq. Ouverture arrondie, test en forme de gaîne droite, conique, triangulaire ou aplatie; loges superposées, légèrement obliques; sommet tronqué sans prolongement.

G. XXVIII. Marginuline, marginulina, Dorb. Orthocère, Lam.

Coq. Ouverture arrondie, située au bout d'un prolongement du sommet de la dernière loge, à son angle antérieur; test en forme de gaîne arquée, sommet déprimé, ayant à son origine l'empreinte volutatoire; loges superposées, légèrement obliques. 
Ce genre renferme un bon nombre d'espèces que $M$. Dorbigny a séparées des Nodosaires et des Orthocères de M. de Lamarck.

G. XXIX. Planulane, planularia, Defr. Astacola? Montf.

Coq. Ouverture arrondie, située au sommet de l'angle extérieur; test très aplati, triangulaire on elliptique, ayant à son origine l'empreinte volutatoire; loges obliques, superposées.

G. XXX. Pavonie, pavonia, Dorb.

Coq. Plusieurs ouvertures aux loges, test déprimé latéralement; loges concentriques.

$2^{\text {e FAMILLE. }}$

LES ÉNALLOSTÉGUES, Dorb.

Anim. Voyez la caractéristique de l'ordre.

Coq. ayant les loges assemblées en tout ou eu partie par alternance, ou empilées sur deux ou trois axes distincts de diverses manières, mais sans former une spirale régulière et nettement caractérisée.

† Alternance des loges totale ou partielle, mais régulière sur deux axes opposés dans une même place, dont les faces sont semblables.

La coquille est toujours d'un tissu poreux, et l'extérieur est rugueux ou couvert d'aspérités.

G. XXXI. Bigénérine, bigenerina, Dorb.

Coq. Loges alternantes seulement dans le jeune âge, ouverture centrale ou marginale, test droit ou arqué, à l'origine cylindrico-conique.

Le changement de mode d'accroissement, dit M: Dorbigny, qui a lieu à un certain âge est une chose qui se re. trouve dans chaque famille.

Ce genre se divise en deux sous-genres.

$$
\mathbf{I}^{\text {ex }} \text { S.-G. Bigénérines proprement dites. }
$$

Ouverture centrale. 


\section{$2^{\mathrm{e}}$ S.-G. Gemmulines, Dorb.}

Ouverture marginale.

G. XXXIL. Textulaine, textularia, Defr.

Coq. Toutes les loges alternantes, ouverture latérale au côté interne de chaque loge et semi-lunaire, test droit, conico-cylindrique, rarement ovoïde.

Ce genre doit à $\mathbf{M}$. Dorbigny l'augmentation d'un très grand nombre d'espèces nourelles, parmi lesquelles plusieurs sont vivantes.

\section{G. XXXIII. Vulvuline, vulvulina, Dorb.}

Coq. Toutes les loges alternantes, ouverture au sommet, en fente ; test droit, ovoïde et déprimé sur ses faces.

计 Alternance totale ou partielle, mais irrégulière; les faces opposées n'étant plus semblables; le plus souvent l'alternance ayant lieu sur trois axes distincts, déterminés par la prépondérance de volume des loges successives et leur extension en recouvrement; une sorte de spirale obscure et peu caractérisée.

Texture vitreuse, très translucide.

G. XXXIV. Dimorphine, dimorphina, Dorb.

Coq. Premières loges seulement alternantes dans la jeunesse, et empilées à la manière des Stichostègues dans l'âge adulte; ouverture ronde au sommet de l'axe.

\section{G. XXXV. Polymorphine, polymorplina, Dorb.}

Coc. Toutes les loges alternantes, ouverture ronde au sommet de la dernière loge.

$\mathbf{I}^{\mathrm{er}}$ S.-G. Polymorphines proprement dites.

Coq. Un grand nombre de loges apparentes, alternance sur deux côfés presque égaux. 
$2^{e}$ S.-G. Guttulines.

Coq. Pas de loges apparentes, alternance sur trois faces, loges embrassantes.

$$
3^{\text {e }} \text { S. -G. Globulines. }
$$

Coq. Alternance sur trois faces, loges embrassantes, trois d'entre elles seulement apparentes.

$$
4^{\mathrm{c}} \text { S.-G. Pyrulines. }
$$

Cog. Alternance irrégulière, ou plutôt une spirale obscure, formée par des loges demi-embrassantes.

Dans le nombre de vingt-huit espèces que renferme ce genre, il y en a beaucoup que $\mathbf{M}$. Dorbigny a observées à l'état vivant.

G. XXXVI. Vrrgurine, virgulina, Dorb.

Coq. Toutes les loges alternantes, ouverture virgulaire et découverte à la partie supérieure de la dernière loge.

G. XXXVII. Sphénoïdrne, spharoidina, Dorb.

Coq. sphérö̈dale, loges en partie recouvrantes, quatre seulement apparentes à tous les âges; ouverture la. térale, semi-lunaire.

\section{3 e FAMILLE.}

LES HÉLICOSTÊGUES, Dorb.

Anim. Voyez la caractéristique de l'ordre.

Coq. ayant les loges assemblées sur un ou deux axes distincts, mais formant une volute spirale, régulière et nettement caractérisée, turriculée ou discoïdale.

Ire Section. - Turbinö̈les, Dorly. Test libre ou fixé; loges empilées sur un seul axe; spire plus ou moins élevéc, apparente d'un côté seulement.

† Spire élevée; test libre. 
G. XXXVIII. Chavuline, clavulina, Dorb.

Coq. Spire très allongée, projetée en ligne droite à un certain âge, et formant alors une suite de loges empilées sur le même axe que celui de la sphère; ouverture terminale et centrale.

G. XXXIX. Uvigérine, uvigerina, Dorb.

Coq. Spire allongée, continue à tous les âges; loges très globuleuses, ouverture centrale, terminale, au bout d'un prolongement de la dernière loge.

G. XL. Bulimine, bulimina, Dorb.

Coq. spirale, turriculée; spire allongée, ouverture virgulaire et latérale près de l'angle supérieur de la dernière cloison.

G. XLI. Valvuline, valuulina, Dorb.

Coq. Spire allongée ou trochoïde; ouverture située près de l'angle ombilical, et fermée en partie par une sorte de lame arrondie, operculaire, et laissant une fente semi-lunaire à découvert.

†† Spire surbaissée.

\section{G. XLIL. Rosaline, rosalina, Dorb. Cidarolle? Montf.}

Coq. fixée par la partie non spirale, trochoïde et régulière; ouverture en fente, située à la région ombilicale et continue d'une loge à l'autre, point de disque ombilical.

\section{G. XLIII. Rotalie, rotalia, Lam.}

Coq. trochoïde et régulière, spire saillante ou déprimée, ouverture en fente longitudinale contre l'avant-dernier tour de spire; pourtour généralement dépourvu d'appendices marginaux, avec ou sans disque ombilical.

Ce genre, très nombreux en espèces vivantes et fossiles, est divisé par M. Dorbigny en sous-genres. 


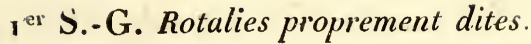

Coq. à ouverture simple, sur la dernière loge, tsochoïde.

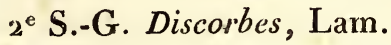

Coq. à ouverture munie de bourrelets, trochoïde; bords carénés.

$3^{\text {e }}$ S.-G. Trochulines, Dorb.

Coq. à ouverture, divisée par un appendice, trochoïde; bords carénés.

$4^{\mathrm{e}}$ S.-G. Turbinulines, Dorb.

Coq. à ouverture continue d'une loge à l'autre, généralement déprimée, à spire surbaissée, non carénée.

G. XLIV. Calcarine, calcarina, Dorb.

Sidérolite, Lam.; Tinopore? et Cortale? Montf.

Coq. Des appendices marginaux rayonnant tout autour de la carène, jamais de disque ombilical, spire souvent masquée, test rugueux ou épineux, ouverture en fente lorigitudinale contre l'avant.-dernier tour de spire.

G. XLV. Grobrgérine, globigerina, Dorb

Coq. libre, trochoïde, irrégulière; spire confuse, formée par des loges sphériques plus ou moins distinctes; ouverture en forme d'échancrure plus ou moins profonde, située vers l'axe de la spire, à l'angle ombilical.

G. XLVI. Gyroïnje, gyroidina, Dorb.

Coq. libre, trochoïde, régulière; spire tronquée, très aplatie; côté opposé très convexe, ouverture en fente longitudinale contre l'avant-dernier tour, mais n'occupant que la partie médiane de la loge. 
G. XLVII. Tromcatuline, truncatulina, Dorb. Hammonia, Sold.; Polyxène et Cibicide, Montf.

Coq. Spire discoïdale, comme tronquée; côté opposé bombé; ouverture en fente, paraissant en dessus, et se continuant en dessous sur la ligne suturale jusqu'à la denxième ou troisième loge, constamment fixée par le côté spiral.

$2^{\mathrm{e}}$ Section. - Ammonoïdes, Dorb. Test libre ou fixé, discoïdal; loges enflées sur un seul axe; tours de spire apparens de chaque côté.

\section{G. XLVili. Planuline, planulina, Dorb.}

Coq. régulière, libre, subturbinée; ouverture en fente contre l'avant-dernier tour de spire; côtés inégaux; spire régulière, plus apparente d'un côté que de l'autre.

\section{G. XliX. Planorbuline, planorhulina, Dorb.}

Coq. fixée, déprimée; côtés inégaux; spire irrégulière, plus apparente d'un côté que de l'autre; ouverture semi-lunaire contre l'avant-dernier tour de spire.

G. L. Operculins, operculina, Dorb.

Lenticulina, Bast.

Coq. libre, régulière; spire régulière, également apparente de chaque côté; ouverture en fente contre l'avant-dernier tour de spire.

\section{G. LI. Soldanie, soldania, Dorb.}

Coq. libre, déprimée; spire régulière, également apparente de chaque côté; ouverture présumée marginale, ou à l'angle extérieur des loges (toutes d'après des figures de Soldani ).

3e Section. -- Nautilö̈les, Dorb. Test libre; loges assemblées sur un ou deux axes distincts, alternantes ou non; spire embrassante en tout ou en partie; point de tours visibles. 
† Loges assemblées sur deux axes ou alternantes, ouverture vers le milieu de la loge.

\section{G. LII. Cassidurine, cassidulina, Dorb.}

Coq. Voyez les caractères ci-dessus.

†† Loges non alternantes ou enfilées sur un seul axe.

a) Côtés inégaux, l'un bombé et l'autre plat.

G. LIII. Anomaine, anomalina, Dorb.

Coq. ayant la méme forme à tous les âges; ouverture latérale en fente, placée contrel'avant-dernier tour de spire; bombée.

G. LIV. Vertébralinz, vertebralina, Dorb.

Coq. très déprimée; spire se projetant à un certain âge, en ligne droite; ouverture en fente, occupant toute la partie supérieure de la dernière loge.

Ce genre ne renferme qu'une seule espèce qui se trouve vivante dans la Méditerranée, la mer Rouge et à Rawack.

b) Côtés inégaux.

I) Plusieurs ouvertures.

G. LV. Poixstomexre, polystomelia, Lam.

Et Vorticiale, Lam.; Andromède, Cellulie, Sporulie, Théméone, Pélore, Géopone et Elphide, Montf.

Coq. déprimée, régulière, ne variant pas dans sa forme, et non ombiliquée; le plus souvent un disque ombilical; ouvertures rondes, disposées sur deux lignes formant un triangle, ou éparses sur les cloisons.

G. LVI. Dendritrnr, dendritina, Dorb.

Coq. régulière, ne variant pas de forme, et ombiliqué; ouvertures ramifiées, le plus souvent réunies en forme de dendrites.

G. LVII. Pénérople, peneroplis, Montf. Et Rénuline, Placentule, Blainv.

Coq. très déprimée, irrégulière, ombiliquée, ув- 
riant de forme selon l'âge; ouvertures disposées sur une ou plusieurs lignes longitudinales.

G. LVIII. Spiroline, spirolina; Lam. Et Lituite, Lam.

Coq. en forme de crosse ; la spire plus ou moins marquée, se projetantà un certain âge en ligne droite, et formant alors un tube cylindrique; dans le jeune âge, la cloison est munie de plusieurs ouvertures, et dans l'âge adulte, d'une seule.

2) Une seule ouverture.

G. LIX. Robuline, robulina, Dorb.

Phonème, Pharame, Héfione, Clisiphonte, Patrocle, Lampadie, Antenore, Robule, Rhinocure, Spinctérule, Montf.

Coq. bombée, un disque central; ouverture marginale ou à l'angle carénal, en fente triangulaire.

G. LX. Cristellaire, cristellaria, Lam. Linthurie, Oreas, Blainv.; Oréade et Scortine, Montf.; Saracénaire, Defr.

Coq. déprimée, ouverture ronde, le plus souvent entourée d'un bourreiet, placée à l'angle carénal des loges.

$\mathrm{I}^{\mathrm{er}}$ S.-G. Cristellaires proprement dites.

Coq. déprimée.

$2^{\mathrm{e}}$ S.-G. Saracénuires, Defr.

Coq: bombée, spire projetée en avant, à un certain âge.

G. LXI. Nontonine, nonionina, Dorb. Macrodite? Itétonie, Cancride, Florilie, Chrysole, Montf.

Coq. à dos arrondi, ouverture en fente contre l'avantdernier tour de spire, apparente à tout âge. 
G. LXII. Numaruare E, nummulina, Dorb. Nummulite et Lenticulite, Lam.; Hélicite, de Blainv., Numulie, Licophre, Rotalie, Egéone, Montf.

Coquille discoïdale, dépourvue d'appendices ; ouverture contre l'avant-dernier tour de spire, masquée dans l'âge adulte.

$\mathrm{I}^{\mathrm{er}}$ S.-G. Nummulines proprement dites.

Coq. ayant les tours de spire embrassant à tout âge.

$$
2^{\mathrm{e}} \text { S.-G. Assilines, Dorb. }
$$

Tours de spire apparens à un certain âge.

G. LXIII. Sidḱroline, siderolina, Lam. Sidérolite, Montf.

Coq. bombée, pourvue d'appendices, en forme d'étoile irrégulière.

\section{$4^{\circ}$ FAMILIE.}

\section{LES AGATHISTĖGUES, Dorb.}

Les Milioles, Fér.

Anim. Voyez la caractéristique de l'ordre.

Coq. ayant les loges pelotonnées de diverses manières sur un axe commun, faisant chacune, dans leur enroulement autour de l'axe, sa longueur totale; ouverture toujours munie d'un appendice, se trouvant alternativement à une extrémité ou à l'autre; texture opaque, blanche.

G. LXIV. Biloculine, biloculina, Dorb.

Coq. ayant les loges embrassantes opposées sur un seul plan, d'eux d'entre elles apparentes.

G. LXV. Spiroloculine, spiroloculina, Dorb.

Coq. ayant les loges non embrassantes, opposées sur un seul plan tout à découvert. 
G. LX VI. Triloculine, triloculina, Dorb.

Coq. ayant les loges opposées sur trois côtés, la mếme forme à tous les âges, trois loges apparentes.

G. LXVII. Articuline, articulina, Dorb.

Coq. ayant les loges opposées sur trois côtés, laissant à un certain âge le mode de pelotonnement, et projetant une ou deux loges cylindriques sur l'axe primitif.

G. LXVIII. Quingueloculine, quinqueloculina, Dorb.

Pollonte? Montf.

Coq. ayant les loges opposées sur cinq côtés; cinq loges apparentes.

G. LXIX. Adelosine, adelosina, Dorb.

Coq. commençant par une grande loge arrondie, ayant un prolongement au bout duquel est une ouverture munie d'un appendice; sur cette première loge viennent se placer des loges en pelotonnement, de manière à former avec l'âge un enroulement sur cinq faces, comme dans les Quinqueloculines.

\section{E FAMILLE.}

LES ENTHOMOSTÈGUES, Dorb.

Anim. Voyez la caractéristique de l'ordre.

Coq. ayant les loges divisées en plusieurs cavités par des cloisons ou par de petits tubes.

† Côtés inégaux.

G. LXX. Amphistégane, amphistegina, Dorb.

Coq. Divisions transversales aux loges, ne paraissant que d'un seul côté de la coquille; quelquefois des divisions parallèles aux loges, paraissant également de chaque côté ; ouverture unique, semi-lunaire du côté le moins bombé et contre le retour de la spire qui est embrassante. 
G. LXXI. Hétérostégine, heterostegina, Dorb.

Coq. Divisions toutes transversales aux loges, visibles également sur chaque côté de la coquille; ouverture contre le retour de la spire.

故 Côtés égaux.

G. LXXII. Orbiculine, orbiculina, Lam. Hélénide, Archidie et Ilote, Montf.

Coq. discoïdale, déprimée dans le sens de l'axe de la spire; un grand nombre d'ouvertures sur des lignes longitudinales; loges partagées en un grand nombre de cavités par des cloisons perpendiculaires et transversales.

G. LXX1II. Alvéoline, alveolina, Dorb.

Alvéolite, Bosc; Orizaire, Def.; Borélie, Clausulie et Miliolite, Montf.; Fasciolite, Park.

Coq. ovoïdale, allongée dans le sens de l'axe de la spire; beaucoup d'ouvertures en lignes transversales; loges partagées en un grand nombre de cavités par des cloisons transversales.

G. LXXIV. Fabulatre, fabularia, Def.

Coq. Loges opposées, pelotonnées sur le même plan et embrassantes, partagées en un grand nombre de tubes longitudinaux, dans le sens de la spire; ouvertures nombreuses, placées alternativement, tantôt à une extrémité, tantôt à l'autre. 


\section{DEUXIËME CLASSE.}

\section{LES PTÉROPODES, Cuvier.}

Aporobranches, Blainville.

Anim. Corps libre et flottant; point de bras pour marcher, ni de pied pour ramper, mais deux nageoires égales et opposées, situées de chaque côté du corps.

Coq. ou non; dans le premier cas, cette pièce est de forme très variable, rarement spirale, toujours cornée ou vitrée, mince et transparente; dans le second cas, elle est remplacée par une tunique ou enveloppe musculeuse.

Les Ptéropodes sont de petits mollusques pélagiens qui ne se montrent sur les rivages que rarement et seulement lorsque les tempêtes ou les courans les y portent. Ils nagent librement au milieu des eaux, et viennent à leur surface dans les instans de calme et surtout au coucher du soleil. Ces petits mollusques sont vifs dans leurs mouvemens, et ils se fixent quelquefois aux corps flottans, tels que les fucus, en les embrassant avec leurs nageoires; mais non comme on l'a pensé, en formant le vide. Nous avons recueilli pendant plusieurs voyages un certain nombre de Ptéropodes nouveaux qui font avec ceux que l'on connaissait déjà le sujet d'un travail particulier: nous allons en donner un court extrait.

I RE FAMILIE.

LES HYALES, hyalea, Fér.

Thecosomata, Blainv.

Anim. muni d'une tête non distincte, avec une troisième membrane natatoire plus petite et intermédiaire, à la partie ventrale; la bouche située dans 
le fond d'une cavité formée par la réunion des organes locomoteurs.

Coq. existant presque toujours, et de forme très variable.

$\dagger$ Un test.

G. I.XXV. Cymbulie, cymbulia, Péron et Lesueur. Anim. oblong, gélatineux, transparent, muni de deux yeux? de deux tentacules? et d'une bouche en forme de trompe? deux nageoires latérales, grandes et arrondies, portent le réseau vasculaire des branchies; elles sont connées à leur base, du côté postérieur, par un appendice intermédiaire en forme de lobe allongé.

Coq. gélatinoso-cartilagineuse, oblongue, en forme de sabot, entièrement revêtue d'une membrane mince ế à peine visible, à ouverture supérieure, longue, tronquée à l'une de ses extrémités.

Ce genre curieux et très incomplétement connu ne contient qu'une seule espèce qui est de la Méditerranée; nous ne la connaissons que par un dessin fait d'après l'animal par M. le baron Cuvier, et que ce savant a bien voulu nous communiquer.

\section{G. LXXVI. Lrmacire, limacina, Cuvier. Spirutella, Blainv.}

Anim. allongé antérieurement, contourné en spirale, en arrière; les branchies en forme de plis sur le dos; la bouche munie de deux petits appendices qui se réunissent, par une de leur extrémité, au bord antérienr.

Coq. très mince, fragile, vitrée, spirale, non carénée, tournant un peu obliquement sur elle-même, à ouverture circulaire et à bords simples.

On ne connait encore qu'une seule espèce de ce genre sur laquelle il serait intéressant d'avoir de nouveaux renseignemens. Elle habite la mer du Nord où l'on dit que, par 
sa prodigieuse quantité, elle peut servir de nourriture aux baleines.

\section{G. LXXVII. HyaLe, hyalea, Lam.}

Cavolina, Abilg.; Tricle, Ocken.; Archonte, Montf. Anim. globuleux ou oblong, muni de deux expansions latérales plus ou moins allongées en arrière; le lobe intermédiaire de forme demi-circulaire; deux tentacules très courts, à peine distincts, contenus dans une gaîne cylindrique; l'ouverture de la bouche pourvue de deux appendices labiaux; l'orifice de l'anus au côté droit du manteau; celui de l'organe mâle, en avant et en dedans du tentacule droit; celui de la vulve, du même côté, au point de séparation des deux parties du corps; les branchies en forme de peigne, de chaque côté, dans une cavité particulière.

Coq. cornée ou vitrée, transparente et fragile, en forme de sabot, droit on recourbé, à ouverture antérieure, et fendue latêralement, tricuspidée en arrière.

Ce joli genre qui a tant intéressé les naturalistes, et dont MM. Cuvier et de Blainville ont fait connaître l'anatomie, est parfaitement distinct de ceux qui l'avoisinent. Les Hyales sont de très petits animaux répandus dans toutes les mers de la zone torride et une grande partie de celles des zones tempérées, et l'on rencontre communément la même espèce dans les points les plus opposés du globe. La découverte que nous avons faite de plusieurs espèces, dont une à l'état fossile, nous a donné occasion de les diviser de la manière suivante.

\section{$y^{\text {er }}$ groupe. Globulosa.}

Coq. subglobuleuse, ayant les fentes latérales presqu'aussi longues qu'elle, les appendices très en arrière. $H$. uncinata, etc.

Ce groupe est le plus nombreux.

$2^{\mathrm{e}}$ groupe. Elongate.

Coq. allongée, ayant les fentes latérales courtes et les appendices avancés. 


\section{H. trispinosa, etc.}

Cinq espèces complètent pour le moment ce groupe.

G. LVXVIII. Cléodore, cleodora, Pér. et Les.

Anim. de forme oblongue ou allongée, muni d'un lobe intermédiaire demi-circulaire, mais n'ayant point d'expansions latérales; le manteau ouvert en avant; les branchies et les organes de la génération incomplétement connus.

Cog. fragile, vitrée, en forme de gaîne ou de cornet plus ou moins aigu postérieurement; l'ouverture très large, presque toujours sans fente et sans appendices latéraux.

La connaissance que nous venons d'acquérir de plusieurs espèces nouvelles de Cléodores et l'étude de leur organisation, nous ont donné lieu de former les sous-genres suivans.

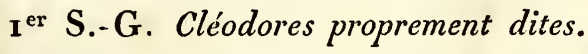

Anim. de forme oblongue, ayant le manteau très dilaté et avancé de chaque côté.

Coq. pyramidale, anguleuse, très dilatée antérieurement, à ouverture fort grande, canaliculée de chaque côté, et rarement fendue.

Ce sous-genre ne renferme que cinq espèces, dont deux sont fort douteuses. La $C$. lanceolata en est le type; c'est la plus grande; nous avons fait connaître son analogue fossile venant du Piémont.

$2^{\text {e }}$ S.-G. Créséis, cresis, Rang.

Anim. très efflé, ayant le manteau non dilaté sur les côtés; les nageoires généralement assez petites.

Coq. très effilée, extrêmement mince, fragile et diaphane, en forme de cornet droit ou recourbé, à ouverture presque toujours anssi large qu'elle, et généralement sans canal; point d'appendices latéraux.

Nous avons formé le sous-genre Créséis pour de très pe- 
tits mollusques nouveaux, que nous arons fréquemment rencontrés an milieu de l'Océan, et auxquels nous réunissons, par analogie, les genres Vaginelle de Dandin et Gradus de Montagu, connus à l'état fossile; nous en comptons déjà neuf espèces.

\section{$3^{\mathrm{e}}$ S.-G. Triptère, tripter, Quoy et Gaimard.}

Anim. oblong, charnu, contractile, muni de deux petites nageoires latérales, et surmonté d'un voile membraneux de même forme et de même grandeur qu'elles.

Coq. diaphane, vitrée, en forme de gaîne cylindrique, arrondie postérieurement, à ouverture circulaire, horizontale et dentelée sur ses bords.

Ce genre est établi sur une seule espèce; nous sommes porté à croire que le voile membraneux décrit par MM. Quoy et Gaimard n'est autre chose que le lobe intermédiaire commun à tous les Ptéropodes de la famille des Hyales, et nous pensons que ce sous-genre devra être réuni au précédent.

G. LXXIX. Cuviérie (i), cuvieria, Rang.

Anim. allongé, muni de deux nageoires assez grandes et d'un lobe intermédiaire demi-circulaire ; les branchies extérieures situées à la partie ventrale et à la base du lobe intermédiaire, l'organe de la génération incomplétement connu, la bouche munie de pièces dentiformes propres à la mastication. Coq. en forme d'étui cylindrique, un peu aplatie près de son ouverture, qui est cordiforme, et dont les bords sont tranchans; le côté opposé à l'ouverture fermé par un diaphragme convexe à l'extérieur,

(I) En établissant ce genre nous avons voulu en faire hommage au célèbre naturaliste qui a institué la classe des Ptéropodes; nous n'avons pas en cela crú déroger à la règle générale, puisque le genre Cuviera, que MM. Péron et Lesueur avaient formé au dépens des Méduses, n'a point été conservé. 
non terminal, étant débordé par les parois du cylindre.

Nous avons établi ce genre curieux sur tne espèce également commune dans la mer des Indes, l'Océan et la mer du Sud; depuis lors, nous en avons reconnu une seconde, mais à l'état fossile, provenant des sables coquilliers du Piémont, où elle a été recueillie par $M$. De Luc père.

\section{G. LXXX. Eunibie, euribia, Rang.}

Anim. muni de deux nageoires horizontales, à la base desquelles est la bouche; le lobe intermédiaire très petit et de forme triangulaire; le corps globuleux, court; les branchies et les organes de la génération inconnus.

Coq. cartilagino-membraneuse, mince, transparente, régulière et en forme de calotte renversée.

Une seule espèce que nons n'avons pu observer suffisamment, mais qui nous a cependant présenté des caractères génériques bien tranchés, nous a donné lieu d'établir ce genre que nous signalons à l'attention des naturalistes.

†† Point de test.

\section{G. LXXXI. Psyché, psyche, Rang.}

Anim. enveloppé d'un manteau membraneux, muni de deux nageoires latérales assez longues, mais ne paraissant point réunies du côté ventral par un lobe intermédiaire; les branchies tapissant les nageoires.

Nous avons établi ce genre sur une espèce des mers de 'Terre-Neuve. M. Reynaud a rapporté de son voyage de l'Inde quelques dessins de mollusques Ptéropodes qui paraissent s'y rapporter.

$2^{\text {e HA MILTE. }}$

\section{LES CLIOS, Fér.}

Gymnosomata, Blainv.

Anim. Tête distincte, point de lobe intermédiaire, mais un ou plusieurs appendices charnus qui le remplacent; une enveloppe ou manteau musculeux. Coq. nulle. 
G. LXXXII. Clio, clio, Brug. Et Cliodites, Quoy et Gaim.

Anim. allongé, enveloppé d'un manteau membraneux, très contractile, portant une tête pourvue de plusieurs tentacules longs, coniques, rétractiles et séparés en deux groupes qui peuvent rentrer à volonté dans de petites cavités qui leur sont destinées; les branchies en forme de réseau vasculaire, tapissant les nageoires; la bouche terminale; des yeux sessiles? trois appendices membraneux à la partie ventrale et correspondant à la base des nageoires; anus et organes de la génération situés dans un tubercule, au côté droit.

Nous réunissons aux Clios les Cliodites de MM. Quoy et Gaimard qui ne paraissent pas en différer. Le genre Clio est peu nombreux en espèces; l'une d'elles, très commune dans les mers du Nord, en est le type. C'est le Clio Borealis qui a servi aux recherches anatomiques de $M$. Cuvier.

G. LXXXIII. Pneumoderme, pneumodermon, Cuv. Anim. oblong, presque cylindrique, divisé en deux parties très distinctes, la postérieure ovale, et l'antérieure conique; les nageoires placées à peu près à la séparation de ces deux parties, et présentant entre elles, et du côté ventral, un petit appendice membraneux; la bouche à l'extrémité d'une sorte de trompe rétractile, ayant à sa base deux faisceaux de tentacules terminés par un petit disque; les branchies situées à la partie postérieure du corps et disposées en forme de deux $\mathrm{C}$ adossés de cette manière $\mathrm{DC}$, et séparés par deux petites barres; anus à droite, et un peu en avant des branchies; orifice des organes de la génération dans un tubercule commun, situé à la racine de la nageoire du côté droit.

Nous avons vu parmi les dessins de M. Reynaud celui d'un mollusque qui paraît tout à la fois voisin des Pneumodermes, et cependant en diffère d'une manière assez remarquable. 


\section{TROISIĖME CLASSE.}

\section{GASTÉROPODES, Cuvier.}

Gastéropodes, Trachélipodes et Hétéropodes, Lam.; Paracéphalophores et Polyplaxiphores, Blainv.

Anim. Corps libre, point de bras pour marcher, mais un pied abdominal, propre à la reptation et dans un petit nombre de cas à la natation; une tête distincte, portant ordinairement une ou plusieurs paires de tentacules; presque toujours des yeux diversement situés sur ou près des tentacules ; des organes respiratoires branchiaux ou pulmonaires, très variables dans leur forme et leur position.

Coq. extérieure, intérieure ou nulle, presque toujours d'une seule piece; conique ou spirale dans le premier cas, et plus ou moins rudimentaire dans le second.

Opercule existant ou now.

Marins, d'eau douce ou terrestres.

Les Gastéropodes forment la classe la plus nombreuse et la plus répandue à la surface du globe; elle comprend, en effet, une grande quantité de mollusques littoraux, plusieurs mollusques terrestres et un bon nombre de pélagiens.

M. Cuvier les a divisés en plusieurs ordres basés sur les différences qu'ils présentent dans la disposition des branchies; nous y ajoutons l'ordre des Nucléobranches pour les Hétéropodes de M. de Lamarck, et celui des Cirrhobranches établi par M. de Blainville pour le genre Dentale.

\section{$I^{\text {er ORDRE. }}$}

NUCLÉOBRANCHES, Blainv.

Hétéropodes, Lam.; fam. des Ptérotrachées, Fér.

Anim. muni d'un pied comprimé en forme de nageoire, avec une ventouse à son bord supérieur; 
des branchies disposées en peigne, les deux sexes sur le même individu.

Coq. Souvent une coquille; spirale, à ouverture très grande, vitrée et très fragile.

Operc. existant quelquefois.

Les mollusques que nous arons déjà, dans un Mémoire anatomique sur le genre Atlante, proposé de rassembler sous le nom de Nucléobranches, emprunté à M. de Blainville, sont tous des animaux pélagiens que l'on rencontre souvent à la surface de l'eau dans les temps calmes, nageant dans une position renversée à l'aide de leur pied comprimé en nageoire. Ils ne rampent jamais; mais ils ont la faculté de se fixer aux corps flottans seuls, en épanouissant sur eux la ventouse de leur nageoire ventrale, et faisant au même instant le vide. Les coquilles qu'ils produisent sont toujours très recherchées dans les collections à cause de leur extrême rareté.

$$
\text { I Te FAMILIE. }
$$

\section{LES FIROLIDES, Rang.}

Ptérotrachées, Fér.; Nectopoda, Blainv.; Urobranchia, Lat. Anim. allongé, droit et horizontal; une ou plusieurs nageoires, branchies formant, avec les autres viscères, un nucleus à la partie dorsale.

Coq., quelquefois; ne pouvant jamais contenir qu'une très petite partie de l'animal.

G. LXXXIV. Frrole, firola, Pér. et Les. Ptérotrachée, Forsk.; Firoloïde et Sagitelle, Les.

Anim. très allongé, gélatineux et transparent, terminé en arrière par une queue plus ou moins longue et pointue; la bouche située à l'extrémité d'une trompe, et renfermant un appareil propre à la mastication? point de tentacules, ou seulement deux rudimens tentaculaires portant les yeux à leur base extérieure; une ou plusieurs nageoires; le nucleus à découvert, protégé seulement par une membrane, et toujours situé au-delà et en arrière de la nageoire ventrale; la terminaison du canal intestinal 
et des organes de la génération dans un tubercule, au côté droit.

Coq. nulle.

Les Firoles sont des animaux très communs dans les mers chaudes et dans celles des zones tempérées; elles se font toujours remarquer par une extrême transparence, souvent interrompue par de nombreuses taches dorées. M. Lesueur, à qui l'on doit des renseignemens sur leur anatomie, en a fait connaître plusieurs espèces, peut-être trop peu distinctes. Il a en outre divisé les Firoles en trois genres, les Firoles, les Firoloïdes et les Sagitelles; les distinctions génériques sur lesquelles ils reposent, ne nous paraissant pas suffisamment justifiées, même pour l'établissement de sous-genres, nous ne les adoptons pas. Le genre Hiptère de M. Rafinesque doit peut-être aussi rentrer dans les Firoles; du reste, nous manquons de détails à son sujet.

Les Firoles sont quelquefois d'une détermination très difficile, à cause des mutilations auxquelles elles sont sujettes; c'est pourquoi on doit mettre la plus grande circonspection dans l'établissement de nouvelles espèces.

\section{G. LXXXV. Garinaire, carinaria, Lamarck. Patella, Lin.; Argonauta, Gmel.}

Anim. gélatineux, transparent, à manteau épais, et toujours couvert d'aspérités (1); terminé en pointe

(1) Nous croyons pouvoir présenter comme caractère constant la présence des aspérités sur le manteau de ces mollusques, parce que nous les avons remarquées sur quatre espèces très distinctes (les $C$. mediterranea et depressa, et deux autres espèces que nous n'avons pu faire connaitre à cause de leur état de mutilation), tandis que nous ne les arons jamais rencontrées dans les Firoles où elles paraissent remplacées par de nombreuses taches. La difficulté où l'on est presque toujours à la mer, de distinguer les Firoles d'avec les Carinaires mutilées, nous oblige à ne négliger aucun des caractères qui peuvent conduire à de plus faciles déterminations; c'est pourquoi nous signalerons encore 
en arrière, et arrondi en avant à la base de la trompe; celle-ci verticale, terminée par la bouche, qui est triangulaire et contient un appareil propre à la mastication, composé de trois lames garnies chacune de rangées de crochets; deux tentacules coniques, allongés et recourbés en avant, portant les yeux à leur base, en dehors, et sur de petits tubercules arrondis; une ou plusieurs nageoires; le nucleus placé dans une cavité, au côté dorsal, sous le bord postérieur de la nageoire ventrale, et protégé par une coquille; la terminaison du canal intestinal et des organes de la génération dans un tubercule, au côté droit.

Coq. extrêmement mince, fragile et transparente; enroulée obliquement sur la droite; à spire très petite, et uniquement au sommet ; à ouverture extrêmement grande et oblongue, divisée en deux parties presque égales par une carène longitudinale.

Les mollusques qui forment ce geure sont de jolis animaux, transparens comme le cristal, et ornés des plus vives couleurs; on ne les rencontre à la surface de la mer que dans les momens de calme, et la plupart du temps mutilés dans quelques unes de leurs parties, surtout au nucleus, ce qui fait que leurs coquilles sont encore si rares dans.les collections. On en connaît quatre espèces bien caractérisées; une seule d'entre elles, la C. vitrée, la plus recherchée, n'a point encore donné lieu de connaître l'animal qui la forme; les autres sont les $C$. fragilis, mediterranea et depressa; cette dernière est nouvelle; nous venons de la décourrir dans les mers de Madagascar.

comme caractère probable la position du peigne branchiale qui nous a toujours semblé placé en avant du nucleus dans les Carinaires, et en arrière dans les Firoles. La position du nucleus par rapport à la nageoire ventrale peut fournir aussi un bon caractère générique. 
$2^{e}$ TAMrLLE.

LES ATLANTIDES, Rang.

Pteropoda, Blainv.; Limacines, Fér.; Procephala, Lat.

Anim. allongé et spiral, une seule nageoire; branchies séparées des autres viscères, et protégées par le manteau.

Coq. pouvant contenir l'animal tout entier. Un opercule.

\section{G. LXXXVI. Atrante, atlanta, Les.}

Corne d'Ammon vivante, Lamanon.

Anim. Corps comprimé latéralement, spiral, portant une nageoire assez grande, foliacée et munie d'une ventouse à son bord postérieur; tête en forme de longue trompe; deux tentacules cylindriques, en avant d'yeux fort gros, comme pédiculés à leur base; bouche à l'extrémité de la trompe ; les organes générateurs mâles, au côté droit, implantés à la base d'un tube très grand qui se termine en avant par l'orifice de l'anus; les branchies en forme de peigne, au plafond de la cavité pulmonaire.

Coq. enroulée longitudinalement, très mince, diaphane, fortement carénée, à ouverture échancrée ou fendue antérieurement, à bord tranchant; spire terminée par un bouton, au fond de l'ombilic, du côté droit.

Operc. vitré, mince, fragile, portant l'impression musculaire dans son centre.

Ce genre extrêmement curieux, sur lequel nous avons publié un Mémoire anatomique dans le Recueil des Mémoires de la Société d'histoire naturelle de Paris, ne contient encore que deux espèces, appartenant à toutes les mers chaudes. 
$2^{e}$ OR DRR.

\section{NUDIBRANCHES, Cuv.}

Tritoniens, Lam.; Polybranches et Cyclobranches, Blainv.

Anim. muni d'un pied pour ramper; des branchies à nu sur quelques parties du dos, toujours symétriques, soit qu'elles se trouvent dans la ligne médiane, soit qu'elles en occupent les côtés; une ou plusieurs paires de tentacules, les deux sexes sur le même individu.

Coq. nulle.

Les Nudibranches sont des animaux tous marins et hermaphrodites; quelques uns d'entre eux habitent les rivages et rampent au moyen d'un pied assez grand; d'autres habitent la haute mer et s'attachent aux fucus par un pied. étroit et allongé, ou bien encore ils nagent dans un sens renversé, le pied à la surface de la mer, s'aidant des bords de leur manteau, de leurs tentacules et de leurs branchies comme de rames.

I Te FAMILLR.

LES PTÉROSOMES, Rang.

Anim. très aplati, pouvu tout autour du corps d'une membrane natatoire horizontale; point de tentacules; les branchies?

Nous établissons provisoirement cette famille pour un mollusque très incomplétement connu, dont M. Lesson a fait le genre Ptérosome; il semble intermédiaire entre les Nucléobranches et les Nudibranches sans pouvoir convenablement entrer dans l'un ou l'autre de ces ordres. Cependant comme il est bien certain qu'il ne peut appartenir au premier, nous le comprendrons dans ce second en attendant que de nouveaux renseignemens viennent nous éclairer à son sujet. 
G. LXXXVII. Ptérosone, pterosoma, Lesson.

Anim. pélagien, gélatineux, transparent, allongé, cylindrique, renflé à son milieu, entouré de deux membranes natatoires, minces, horizontales, prenant naissance à la queue, se continuant en conservant une forme ovalaire au-delà de la tête, où elles s'unissent en avant de la bouche; bord antérieur plus épais, et comme tronqué, le postérieur rétréci et plus mince; bouche terminale, sans trompe; yeux sessiles, oblongs, rapprochés; queue cylindrique, pointue; les organes digestifs paraissant à travers la substance de l'animal; les branchies, l'anus et les organes de la génération inconnus.

M. Lesson a découvert ce mollusque dans les mers de l'équateur entre les Moluques et la Nouvelle-Guinée, où il se montrait en assaz grande abondance; ce naturaliste en a donné une jolie figure dans les Mémoires de la Société d'histoire naturelle. D'après ses observations, il se sert avec avantage de la membrane dont il est entouré pour se mouvoir , et nage horizontalement avec une grande vivacité.

\section{FAMILLE.}

LES GLAUQUES, Fér.

Tritoniens, Lam.; Tétracères, Blainv.; Phyllobranches, Lat.

Anim. muni de deux paires de tentacules, et quelquefois de trois; les branchies en forme de lanières ou de cirrhes.

G. LXXXVIII. GLAUQUe, glaucus, Forster.

Anim. pélagien, gélatineux, allongé, un peu aplati et terminé en pointe, en arrière; le pied très étroit et presque rudimentaire, la tête distincte, munie de quatre tentacules très courts, aplatis et triangulaires; la bouche subterminale; les branchies disposées par paires sur les côtés, et propres à la natation, étant formées par des palettes oblongues, 
entourées d'appendices digités; la terminaison des organes de la génération dans un tube commun, à la partie antérieure du côté droit ; anus du même côté, plus en arrière.

Les Glauques sont de charmans petits mollusques ornés des plus riches couleurs; ils nagent lentement à la surface de la mer à l'aide de leurs branchies et toujours le dos en bas. On les rencontre souvent dans l'Océan et la Méditerranée par essaims nombreux, et quand ils éprouvent quelque souffrance on les voit se contracter et se rouler en cercle; exposés hors de l'eau, ils se décomposent promptement.

On ne connaissait encore que le G. hexapterygius : nous donnons le nom de tetrapterygius à une nouvelle espèce de l'Océan qui ne porte que deux paires de branchies.

\section{G. LXXXIX. Lanrogère, laniogerus, Blainv.}

Anim. Corps à peu près de même forme que celui des Glauques, épais et plus large en avant, plus étroit et plus mince en arrière; gastéropode, pourvu de chaque côté d'une série de lames molles finement pectinées, divisée en deux parties ; bouche et tentacules comme dans les Glauques, ainsi que la terminaison des appareils de la digestion et de la génération.

M. de Blainville a établi ce genre sur un individu, le $L$. elfortii, de la côllection du Muséum britannique; la figure qu'il joint à sa description nous rappelle les Glauques que nous avons conservés dans l'esprit de vin, et qui, devenus plus gonflés, comme il leur arrive toujours à leur mort, ont en même temps perdu quelques unes de leurs lanières branchiales.

\section{G. XC. Briarée, briarcea, Quoy et Gaim.}

Anim. pélagien, gélatineux, transparent, scolopendriforme, aplati, ayant deux yeux? sessiles et quatre tentacules courts, larges et triangulaires, les postérieurs portant à leur extrémité deux sortes 
d'antennes très longues, filiformes, élas tiques et résistantes; terminé en arrière par une queue; branchies disposées de chaque côté, et représentées par des lames aplaties, bifurquées à leur extrémité, et décroissantes dans leur longueur, de la tête à la queue, où elles disparaissent insensiblement; terminaison du canal intestinal et des organes de la génération inconnue?

Le genre Briarée est certainement une des découvertes les plus intéressantes de MM. Quoy et Gaimard. Ce mollusque, quoiqu'incomplétement connu, ne peut laisser d'incertitude sur la place qu'il doit occuper; car si ses caractères ne suffisaient pas pour le faire reconnaitre, on pourrait y être conduit par l'observation de cette disposition circulaire qu'il prend dans l'état de souffrance, et qui rappelle si bien le genre Glauque à ceux qui l'ont observé vivant.

Le B. scolopendra est la seule espèce connue; elle a été trouvée dans les eaux du détroit de Gibraltar.

\section{G. XCI. ÉoLIDe, eolidia, Cuv.}

Anim. pélagien, limaciforme, gélatineux ; tête distincte, munie de deux ou trois paires de tentacules; pied entier et occupant presque toute la longueur de l'animal ; branchies formées par des cirrhes aplaties ou coniques, disposées par rangées sur la partie supérieure du corps; organes de la génération réunis dans un même tubercule, au côté droit et en avant; anus un peu plus en arrière.

Ce genre se compose d'une grande quantité de petits animaux pélagiens et littoraux dont les espèces sont généralement assez mal décrites ou figurées. Les masses de fucus natans que l'on rencontre si fréquemment entre les tropiques, en offrent toujours beaucoup, et il est facile de les reconnaître à leur forme allongée et rampante, ainsi qu'aux branchies tentaculiformes dont ils sont tout hérissés; ils ne nagent point; cependant ils viennent se suspendre à la surface de l'eau, le pied en haut, et s'y meuvent assez bien par le moyen d'ondulatious précipitées. 
Nous partageons ce joli genre en deux sous-genres bien distincts.

${ }^{\text {er }}$ S.-G. Eolides proprement dites.

Les branchies disposées par anneaux transverses, distans les uns des autres.

$$
2^{\text {e }} \text { S...G. Cayolines, Brug. }
$$

Les branchies disposées par séries longitudinales, de chaque côté de la ligne médiane.

\section{G. XCII. Tergipe, tergipes, Cuv. Aeolis, Ock.; Tergipède, Blainv.}

Anim. limaciforme, gélatineux; la tête assez distincte et munie de deux paires de tentacules; le pied entier et occupant presque toute la longueur; les branchies en forme de petites massues, peu nombreuses, disposées sur deux rangées, une de chaque côté du dos; terminaison du canal intestinal et des organes de la génération comme dans les éolides?

Les Tergipes paraissent être très voisins des Éolides avec lesquelles on les réunirait peut-être, si on les connaissait mieux. M. Cuvier indique bien, il est vrai, comme caractère particulier, que chaque organe branchiale est terminé par un petit suçoir, pouvant leur servir comme de pied pour marcher sur le dos, mais nous pensons que cette singulière organisation demande à être confirmée par de nouvelles observations faites sur l'animal vivant.

\section{3e FamitLE.}

\section{LES TRITONIES, Fér.}

Tritoniens, Lam.; Dicères, Blainv.; Séribranches, Lat.

Anim. Deux tentacules supérieurs, rétractiles, dans une sorte de gaîne située à leur base; un voile membraneux plus ou moins étendu au-dessus de la bouche; organes de la génération et anus distans, 
au côté droit; organes de la respiration sous diverses formes, mais disposés sur deux rangées longitudinales.

\section{G. XCIII. Théthys, thethys, Linné.}

Anim. pélagien, gélatineux et transparent; la tête distincte et comprenant un large voile membraneux, frangé, formant l'entonnoir, se raccourcissant en dessous, et du milieu duquel s'élève une petite trompe terminée par la bouche; les tentacules au nombre de deux, situés à la base du voile, comprimés, ouverts à leur sommet pour donner issue à un petit tube conique et rétractile; pied très grand, branchies formées par deux séries longitudinales de houppes rameuses, alternativement inégales de droite à gauche, et de l'avant à l'arrière; organes de la génération réunis au côté droit antérieur, orifice de l'anus plus en arrière.

Ce genre curieux sur lequel M. Cưvier a jeté de si grandes lumières, paraitt ne renfermer encore qu'une seule espèce, le T. fimbria, qui est de la Méditerranée; il habite loin des rivages sur les bancs madréporiques ou les plantes errantes, et se sert de son voile pour la natation.

\section{G. XCIV. MéLIbéz, melibe, Rang.}

Anim. pélagien, gélatineux, transparent et limaciforme; la tête distincte et comprenant un voile membraneux, contourné en forme d'entonnoir, garni intérieurement de cirrhes dirigés à l'extérieur, et du milieu duquel s'élève une petite trompe terminée par la bouche; tentacules au nombre de deux, situés à la base du voile, très allongés, coniques, terminés par une petite capsule, de laquelle sort un organe conique et rétractile; pied aussi long que l'animal, mais extrêmement étroit, en forme de sillon; branchies formées de deux séries peu nombreuses de massues oblongues, arrondies à leur sommet, pédiculées à leur base, et recouvertes 
de petits tubercules; organes de la génération réunis au côté droit antérieur, anus plus en arrière.

Ce mollusque est sans contredit très voisin des Théthys avec lesquelles nous l'avions d'abord confondu; mais l'étude que nous avons faite depuis de ce dernier, sur un individu vivant, nous a engagé à leur affecter une différence générique fondée sur l'organisation toute différente des branchies. La Mélibée, qui nage très bien en agitant de côté et d'autre la partie postérieure de son corps, habite sur des plantes errantes dans les mers du cap de BonneEspérance, où nous avous pu l'observer vivante pendant assez long-temps. Nous avons remarqué que les branchies tombaient facilement pour peu qu'on les touchât. L'espèce qui sert de type au genre est la $M$. rosea. Peut-être faudra$\mathrm{t}$-il y joindre quelques petits animaux découverts par M. Dorbigny sur les côtes de La Rochelle, et qui présentent pour organes de la respiration de petites massues disposées sur deux rangées longitudinales et dorsales.

\section{G. XCV. Scyllée, scyllaea, Lin.}

Anim. pélagien, gélatineux, très comprimé latéralement; à tête peu distincte, le voile rudimentaire et en forme de fer à cheval; deux tentacules, grands, en forme de cornets renversés, aplatis, fendus en avant, ouverts au sommet pour donner passage $\dot{a}$ un petit corps pointu et rétractile; bouche à l'extrémité d'une très petite trompe, armée d'un appareil propre à la mastication; pied long et très étroit, en forme de sillon; branchies en forme de petits pinceaux touffus, épars sur les faces internes de plusieurs appendices pairs de la peau, et sur une crête caudale et médiane; orifice de l'anus et des organes de la génération, au côté droit.

Les Scyllées sont des animaux extrêmement répandus dans toutes les mers chaudes, et que l'on trouve surtout parmi les feuilles des fucus natans; aussi leur pied, comme 
celui de presque tous les animaux de cette famille, n'est-il propre qu'à embrasser les tiges de ces plantes. On ne connait encore que les $S$. pelagica et fulva.

\section{G. XCVI, Tritone, tritonia, Cuv.}

Anim. limaciforme, à tête peu distincte, surmontée de deux tentacules rétractiles, dans une sorte d'étui, portant un voile arqué, frontal, peu étendu; la bouche armée de deux màchoires latérales, cornées, tranchantes et denticulées sur les bords; le pied long et canaliculé; branchies en forme de houppes rameuses, rangées en série longitudinale, de chaque côté du dos; organes de la génération réunis au côté droit antérieur; anus situé plus en arrière.

Les Tritonies, comme on en peut juger par la disposition de leur pied, sont des mollusques qui s'attachent particulièrement aux plantes marines qu'elles ne sauraient quitter pour nager; elles sont très voisines des Scyllées dont elles ont entièrement les mœurs et en grande partie l'organisation; les espèces ne sont pas encore bien déterminées; cependant il en existe plusieurs sur nos côtes, parmi lesquelles on doit distinguer la $T$. hombergii qui a servi aux recherches anatomiques de $M$. Cuvier.

$$
4 \text { e FAMiLLE. }
$$

\section{LES DORIS, Fér.}

Tritoniens, Lam ; ordre des Cyclobranches, Blainv.; Urobranches, Lat.

Anim. muni de quatre tentacules, dont deux supérieurs et deux inférieurs, sous le rebord du manteau; les organes de la respiration semblables à de petits arbuscules, et formant dans la ligne médiane un bouquet autour de l'anus.

G. XCVII. Polycìre, polycera, Cuv.

Anim. limaciforme, charnu, à manteau à peine débordant; tête peu distincte, couronnée d'un nombre 
toujours pair d'appendices tentaculiformes; tentam cules supérieurs en forme de massue, contractiles dans une cavité; pied long, assez étroit, et quelquefois canaliculé; les branchies groupées autour de l'anus, dans la ligne médiane, un peu en arrière, et composés d'organes simples ou rameux, souvent très étendus et divisés, quelques appendices membraneux les environnant; orifice des organes de la génération au côté droit.

Les Polycères sont certainement très voisins des Doris; mais c'est à tort, selon nous, qui avons pu fréquemment observer ces deux genres à l'état de vie, que quelques auteurs les ont réunis; elles vivent généralement sur les plantes marines, rampant peu, avec lenteur, et nageant avec quelque facilité. On n'en a encore fait connaître qu'un petit nombre d'espèces; il en existe beaucoup d'autres, si nous en jugeons par celles que nous avons vues dans différentes collections.

\section{G. XCVIII. Don is, doris, Cuv.}

Anim. charnu, oblong, planulé ou prismatique; recouvert d'un large manteau dépassant très souvent le pied et la tête; quatre tentacules, dont deux supérieurs, en forme de massue, contractiles dans une cavité, et deux inférieurs, coniques, sous le rebord antérieur du manteau; bouche à l'extrémité d'une très petite trompe, armée d'une éminence linguale hérissée de denticules; le pied oblong, allongé, généralement assez. grand; les branchies en forme d'arbuscules réguliers et rayonnans autour de l'orifice de l'anus; celui-ci situé dans la ligne médiane, un peu en arrière; orifice des organes de la génération sous le rebord droit du manteau.

Le genre Doris paraît être le plus nombreux en espèces de tous les mollusques sans coquilles; elles se rencontrent en effet sur tous les rivages, car ce sont des animaux essentiellement gastéropodes. Leur forme oblongue ct toujours un peu aplatie, et la singulière disposition de leurs 
branchies les rendent très reconnaissables. Généralement elles montrent de belles couleurs et des ornemens bien própres à établir des distinctions spécifiques, aussi en a-t-on fait à différentes fois des monographies fort intéressantes; M. Cuvier, à qui l'on doit une connaissance très approfondie de l'organisation des Doris, en a, le premier, donné une assez considérable, et dans laquelle il propose déjà d'établir les deux groupes suivans, qui, reproduits tout récemment par M. Rapp de Tubingen, ont servi pour la distribution d'un nombre d'espèces double du sien. Outre cela nous pouvons annoncer que MM. de Férussac et Dorbigny préparent une monographie de ce genre qui sera encore beaucoup plus étendue.

jer groupe.

Espèces à manteau ovale, débordant le pied.

D. verrucosa, etc.

$$
2^{\mathrm{e}} \text { groupe. }
$$

Espèces à corps prismatique, à manteau presque aussì étroit que le pied.

D. lacera, etc.

G. XCIX. Onchroore, onchidoris, Blainv.

Anim. de forme ovalaire, bombé en dessus; quatre tentacules comme dans les Doris, outre deux appendices labiaux; le pied ovale, épais, dépassé dans toute sa circonférence par les bords du manteau; organes de la respiration formés par des arbuscules très petits, disposés circulairement, et contenus dans une cavité située à la partie postérieure et médiane du dos; anus également médian, mais placé à la partie inférieure et postérieure du rebord du manteau; les orifices des organes de la génération très distans, et réunis entre eux par un sillon extérieur occupant toute la longueur dủ côté droit.

M. de Blainville a établi ce genre sur un individu de la collection du Muséum britannique qui, selon l'usage de 
cet établissement, ne portait point d'indication de patrie; c'est l'O. leachii.

\section{5e FAMILLE.}

\section{LES PLACOBRANCHES, Rang.}

Anim. portant quatre tentacules, et muni de deux expansions membraneuses, latérales, propres à la natation, tapissées supérieurement, ainsi que toute la surface du dos, de lamelles branchiales.

\section{G. C. Pracobranche, plancobranchus, Van Hasselt.}

Anim. oblong, cylindracé, charnu, à manteau dilaté de chaque côté en deux lobes ou nageoires membraneuses, demi-circulaire, embrassant toute la longueur, depuis le cou jusqu'à l'extrémité postérieure, et pouvant se croiser sur le dos, en formant un canal intérieur, ouvert aux deux extrémités; tête peu distincte du corps, déprimée, et portant sur son sommet deux yeux petits et rapprochés; deux paires de tentacules, coniques, peu allongés, la paire inférieure un peu aplatie et triangulaire; bouche inférieure, fendue en long, et munie de chaque côté d'un appendice lamelleux; pied long, réuni au manteau; branchies tapissant toute la face supérieure des lobes et du dos, en forme de lamelles minces, serrées, longitudinales, et partant d'un centre commun situé à la partie antérieure; anus situé au côté droit antérieur, orifice des organes sexuels séparés, celui de l'ovaire un peu en avant de l'anus, et celuj de la verge à la base du tentacule droit.

Le genre Placobranche est établi par feu Van-Hasselt pour un mollusque extrêmement curieux, que ce courageux naturaliste, si digne de nos regrets, a découvert à l'île de Java peu de temps avant sa mort. La description et les détails dans lesquels il est entré à son sujet, seraient certainement très suffisans pour beaucoup d'autres mollusques; 
mais ils laissent encore quelque chose à désirer pour celui-ci qui présente une organisation si différente de ce que nous connaissons jusqu'à présent. Nous devons à l'obligeance de M. de Férussac quelques individus du Placobranche, mais qui sont malheureusement mal conservés, et ne nous ont pas permis de pousser nos recherches beaucoup plus loin que ne l'a fait Van-Hasselt; ils nous mettent cependant à même de reconnaître l'exactitude de ses observations. D'après le concours des détails obtenus par ce naturaliste, de la vérification que nous en avons faite et de quelques caractères de plus que nous avons pu saisir, nous croyons devoir, comme lui, placer ce mollusque dans l'ordre des nudibranches, et en faire une famille à part, conduisant aux Inférobranches et aux Tectibranches.

Le Placobranche, à l'exemple des Aplysies, redresse et croise ses lobes sur le dos, et forme ainsi un canal ouvert par les deux bouts, dans lequel l'élément ambiant circule pour frapper les branchies dont il est tapissé. L'espèce qui a servi de type à ce genre, et qui a au plus deux pouces de long, est le $P$. ocellatus.

\section{$3^{e}$ ORDRE.}

\section{INFEROBRANCHES, Cuv.}

Gast. Phyllidiens et semi-Phyllidiens, Lam.; Inférobranches et fam. des Subaplysiens, Blainv.

Anim. muni d'un pied pour ramper, toujours très grand; des branchies en forme de longue suite de feuillets, à la partie inférieure du corps, entre le rebord avancé du manteau et celui du pied, soit tout autour du corps, soit à droite seulement ( $I$ ); les organes de la génération toujours sur le même individu, une ou deux paires de tentacules.

Quelquefois une coquille interne ou externe.

Les Inférobranches sont tous marins, à l'exception du

(I) Le genre Ancyle étant sénestre, les branchies, dans ce seul cas, sont situées à gauche. 
genre Ancyle, que nous y introduisons provisoirement et qui est fluviatile et hermaphrodite. Ils habitent les rivages, et ne peuvent s'en écarter, car ils sont essentiellemont gastéropodes, et ne nagent jamais.

\section{I re FAMILLE.}

LES PHYLLIDIENS, Lam.

Phyllidies, Fér.; ordre des Inférobranches, Blainv.; Bifaribranches, Lat.

Anim. portant les branchies également des deux côtés. Coq. nulle.

G. CI. Phyluidie, phyllidia, Cuv.

Anim. ovale, oblong, convexe en dessus, à manteau coriace, épais, formant un rebord saillant autour du corps; tête petite, cachée sous son rebord antérieur; bouche en forme de petite trompe, munie d'une masse linguale denticulée; quatre tentacules, deux supérieurs, rétractiles dans de petites cavités, et deux inférieurs et labiaux; pied ovale, allongé; branchies en forme de lamelles obliques, formant, sous le rebord du manteau, un cordon tout autour du corps; anus à la partie postérieure et médiane du manteau; orifices de la génération dans un tubercule commun, au côté droit antérieur.

\section{G. CII. Diphylume, diphyllidia, Cuv. Linguelle, Blainv.; Arminia, Rafi.?}

Anim. de forme ovale, très déprimé; le manteau débordant le pied de toutes parts, excepté en avant, où la tête reste à découvert; les tentacules au nombre de deux seulement? le cordon branchial n'occupant que les deux tiers postérieurs du rebord inférieur du manteau; anus inférieur, au côté droit postérieur ; organes de la génération dans le même tubercule, au côté droit antérieur. 
Le genre Diphyllidie a été établi par M. Cuvier pour un mollusque très voisin des Phyllidies, et qui, au rapport de M. de Blainville, serait le même que son genre Linguelle; il paraitrait anssi que le genre Arminia de M. Rafinesque n'eu diffère pas. D'après cela il convient donc de ne le comprendre que sous la dénomination qui lui a été affectée la première, celle que nous lui conservons.

\section{$2^{\text {e FAMIIILE. }}$}

\section{I.ES SEMI-PHYLLIDIENS, Lam.}

Ombrelles et Pleurobranches, Fér.; Patellö̈des et Subaplysiens, Blainv.; Unabranches, Lat.

Anim. portant les branchies au côté droit seulement (à l'exception du genre Ancyle, qui est sénestre). Quelquefois une coquille interne ou externe, et, dans ce dernier cas, recouvrante.

\section{G. CIII. Ancrle, ancylus, Geoffroy.}

Anim. ovale, en cône légèrement recourbé en arrière, ayant le manteau peu ample, ne recouvrant point la tête, et mince sur les bords; tête très grosse, munie de deux tentacules, gros, cylindriques, contractiles, oculés à leur base interne, et avoisinés au côté externe par un appendice foliacé; bouche inférieure, avec quelques apparences d'appendices labiaux de chaque côté; pied elliptique, grand; branchies dans une sorte de cavité, au milieu du côté gauche, entre le pied et le manteau; anus au côté gauche.

Coq. mince, recouvrante, presque symétrique, en cône oblique, en arrière; à base ovale plus ou moins allongée, à soinmet pointu, non marginal, un peu incliné à droite.

Ce genre n'a cessé d'être ballotté d'une famille dans une autre, et peut-être le sera-t-il encore malgré ce que l'on 
sait de son animal. Quoi qu'il en soit, il ne peut plus être classé avec les autres genres de mollusques avec lesquels il vit dans les eaux douces, puisqu'il est branchifère, et que ceux-ci sont au contraire pulmonés. Nous le plaçons en attendant dans la famille des Semi-Phyllidiens où la disposition de ses branchies semble l'appeler, en faisant remarquer toutefois qu'au lieu de les avoir à droite comme les Pleurobranches, il les a à gauche; mais ce caractère est de peu d'importance, l'animal paraissant sénestre, comme plusieurs mollusques d'eau douce, puisque l'anus s'ouvre également à gauche.

Les Ancyles vivent dans les eaux douces, des fontaines et des ruisseaux; ils rampent sur les pierres ou sur les plantes aquatiques; nous ne les avons jamais vus respirer l'air en nature.

G. CIV. Pleurobranchée, pleurobranchaca, Meckel. Pleurobranchidie, Blainv.

Anim. ovale, allongé, plat en dessous, convexe en dessus, pointu en arrière; aucun indice de manteau, seulement une légère expansion de la peau, longue et étroite, au milieu du côté droit; tête très grosse, et portant la bouche à l'extrémité d'une trompe; deux paires de tentacules auriformes, les antérieurs à l'extrémité d'un bandeau musculaire transverse, frontal; les postérieurs un peu plus en arrière, et fort séparés l'un de l'autre; pied très grand, plus étendu en arrière qu'en avant; une seule branchie fixée au côté droit, et entièrement à découvert; la terminaison des organes de la génération dans un tubercule commun, en avant des branchies; l'anus en dessus de celles-ci et au milieu de leur longueur.

Le genre Pleurobranchée est extrêmement voisin des Pleurobranches, car il n'en diffère que par l'absence du manteau, la disposition des tentacules et la place qu'occupe l'orifice de l'anus qui est plus en avant que dans les Pleurobranches; aussi nous pensons que l'on pourrait, sans incon- 
vénient, en faire une division de ceux-ci. M. de Blainville, à qui nous empruntons les caractères du genre Pleurobranchée qu'il a pu observer par lui-même, pense que c'est le Pleurobranche baléarique de Delaroche, et le type du genre Cyanogaster de M. Rudolphi. La seule espèce connue est la P. meckeli.

\section{G. CV. Pleurobranche, pleurobranchus, Cuv.}

Et Lamellaire, Montagu, ou Berthelle, Blainv.

Anim. oblong, charnu, convexe en dessus, à manteau très grand et débordant; pied grand, débordant également, et laissant par cette disposition un large canal tout autour $\mathrm{du}$ corps; tête distincte, munie d'un voile s'unissant de chaque côté avec les bords du pied, et de deux tentacules tubuleux et fendus antérieurement; bouche à l'extrémité d'une trompe; branchies composées d'une double série de lamelles formant un panache au côté droit postérieur, entre le manteau et le pied; anus porté par un petit tube en arrière des branchies, organes de la génération en avant.

Coq. Quelquefois un test rudimentaire, membraneux, à sommet assez distinct, caché dans l'épaisseur du manteau.

Le genre Pleurobranche est très nombreux en espèces; mais peu d'entre elles sont décrites. Nous lui réunissons le genre Berthelle de M. de Blainville, qui n'est autre que le Lamellaire de Montagu, et qui ne diffère des Pleurobranches en rien d'essentiel.

Nota. Nous arons vu dans la belle série des dessins que MM. Quoy et Gaimard, qui viennent de terminer d'une manière si glorieuse leur second voyage autour du monde, ont envoyés à l'Institut pour y être gardés en dépôt, deux genres nouveaux proposés par ces naturalistes, sous les noms de Westeruia et de Gervisia pour de jolis petits animaux très voisins des Pleurobranches, et qui augmenteront, sans doute, la famille des Semi-Phyllidiens. 
G. CVI, Oмbrelle, umbrella, Lam. Gastroplax, Blainv.; Acarde, Megerle.

Anim. de forme oblongue, très déprimé, bombé en dessus, très plat en dessous, et charnu; le manteau peu étendu, la tête non distincte, la bouche située dans le fond d'une échancrure étroite et profonde, en avant du pied, celui-ci à bords épais et relevés tout autour; quatre tentacules, deux supérieurs, tronqués, fendus, comme lamelleux à l'intérieur, deux plus petits, en forme de crètes pédiculées, de chaque côté de la bouche ; pied extrêmement grand, débordant de toutes parts, lisse et plat; branchies foliacées, disposées en cordon tout le long du côté droit, et se portant même un peu à gauche, en passant par-devant; anus en forme de petit tube, en arrière des branchies; organes de la génération très rapprochés, situés à droite et en avant.

Coq. externe, calcaire, solide, très déprimée, presque toute plate, irrégulièrement circulaire, un peu convexe en dessus et concave en dessous, à sommet excentrique, conique et légèrement infléchi, à bords tranchans, à stries concentriques et rayonnantes; fixée à la partie dorsale qu'elle recouvre.

Nous décrivons l'animal de l'Ombrelle d'après l'individu que M. Reynaud a déposé au Muséum d'histoire naturelle, et qui malheureusement se trouve privé de sa coquille. Quoi qu'il en soit, on ne peut douter qu'elle ne fût fixée à la partie supérieure du mollusque, au-dessus des branchies, car on y retrouve encore l'empreinte et des portions évidentes des muscles d'attache. M. de Blainville, qui, le premier, a observé ce mollusque curieux, l'avait désigné sous le nom de Gastroplax, pensant que la coquille était fixée sous le pied, comme cela était à ce qu'il parait, mais artificiellement, sur l'individu qu'il observait. Depuis lors, il a rectifié cette erreur que notre observation vient aussi détruire. A l'exception de la position de la coquille, les figures que ce savant donne dans le Dictionnaire des Sciences 
naturelles 6out très bonnes. On ne connaft encore que deux espèces de ce genre, l'une des mers de l'Inde, l'autre de la Méditerranée. M. Peyraudeau a trouvé celle-ci sur les côtes de la Corse.

\section{G. CVII. Sprricelle, spiricella, Rang.}

Anim. inconnu.

Coq. très aplatie, allongée, arquée, à bords tranchans; sommet spiral, sénestre, contourné horizontalement, situé en arrière et à gauche, ouvert à la face inférieure; une impression peu distincte, mais paraissant occuper la partie postérieure de la coquille, où elle se montre à peu près parallèle au bord.

Nous arons établi le genre Spiricelle dans le Bulletin du 23 décembre r 828 de la Société linéenne de Bordeaux pour une petite coquille fossile, qui a été trouvée dans les Faluns de Mérignac par M. Charles Des Moulins. Bien examinée, elle présente de grands rapports avec le genre Cabochon. Cependant nous l'en avons beaucoup écarté, parce que d'une part elle présente des caractères vraiment distincts, tels que d'avoir les bords de son ouverture extrêmement dilatés de manière à former une vaste surface oblongue qui lui sert de base, et d'avoir le sommet tourné horizontalement et non tout-à-fait postérieur, et que de I'autre elle nous paraît avoir uppartenu à un mollusque plus grand qu'elle qui ne l'avait produite que pour abriter une seule partie de son corps, les branchies par exemple.

Nous n'en connaissons qu'une seule espèce, c'est la Spiricella unguicuius.

\section{G. GVIII. Siphonarre, siphonaria, Sowerby.}

Anim. ovale, subdéprimé; la tête subdivisée en deux lobes égaux, sans tentacules, ni yeux évidens; les bords du manteau crénelés, une branchie en forme de membrane carrée, dans le sinus formé à droite, entre le pied et le manteau.

Coq. patelloîde, elliptique, à sommet bien marqué, 
un peu gauche et postérieur ; une espèce de canal ou de gouttière sur le côté droit ; impression musculaire en forme de fer à cheval, le lobe droit partagé en deux par le canal.

Nous empruntons la caractéristique de ce genre à $M$. de Blainville, car nous ne connaissons pas l'animal des Siphonaires. Nous observerons en même temps que nous avons vu de jeunes Patelles avoir le caractère des Siphonaires, et en conserver encore des traces dans un âge plus avancé. Ce n'est donc que provisoirement que nous adoptons ce genre, et que nous lui assignons une place parmi les Inférobranches.

\section{$4^{e}$ ORDRE.}

\section{TECTIBRANCHES, Cuv.}

Gastéropodes laplysiens et bulléens, Lam.; Monopleurobranches, Blainv.

Anim. muni d'un pied pour ramper; des branchies sur le dos, un peu à droite, généralement protégées par quelques expansions du manteau; les organes de la génération sur le même individu, mais toujours distans au côté droit antérieur, et liés entre eux par un sillon extérieur.

Coq. Très souvent une coquille; externe ou interne.

Les Tectibranches sont des mollusques généralement répandus sur les rivages, où on les voit ramper, mais qui, pour la plupart, jouissent en même temps de la faculté de nager, à l'aide de larges expansions latérales. Ils sont tous marins et hermaphrodites.

\section{TE FAM ILLE.}

\section{LES APLYSIENS, Blainv.}

Laplysiens, Lam.; Dicères, Fér.; Tentaculés, Lat.

Anim. non divisé, muni de quatre tentacules oculés à leur base antérieure, et quelquefois d'expansions membraneuses latérales, propres à la natation, les branchies en forme de panache, dans une cavité 
dorsale, protégée la plupart du temps par un opercule libre au côté droit, ou simplement par les bords rapprochés du manteau; les organes de la génération très distans.

Coq. rudimentaire ou nulle.

A. Quatre tentacules.

G. CIX. Aplysin, aplysia, Rang.

Lepus marinus des anciens; Laplysia, Lin.; Dolabella, Lam.; Notarche, Cuv.

Anim. rampant, oblong, convexe en dessus, plat en dessous, allongé en avant, aigu en arrière; portant deux expansions latérales du manteau, renversées sur le dos, quelquefois très amples, et alors propres à la natation; tête distincte; bouche située au-dessous, fendue en long, et armée de pièces propres à la mastication; tentacules antérieurs, élargis; tentacules postérieurs, coniques et fendus en long; pied grand et calleux; le peigne des branchies renfermé dans une cavité dorsale protégée presque toujours par un opercule; l'anus en arrière des branchies, à l'intérieur du siphon; orifice des œufs un peu en avant et à droite du peigne branchial; organe mâle tout-à-fait en avant, sous le tentacule droit antérieur.

Coq. Quelquefois une coquille, rudimentaire, toujours interne, servant à consolider l'opercule.

Les Aplysies sont des animaux rampans, dont une grande partie jouit en outre de la faculté de nager, au moyen des larges expansions de leur manteau. Elles habitent tous les climats, et on les trouve généralement sur les rivages, parmi les rochers et les plantes marines. Nous avons publié récemment une monographie de la famille des Aplysiens, dans laquelle ce beau genre est traité avec détail, et augmenté, par nos découvertes, du triple de ce qu'il était.

Voici les divisions que, d'après de nombreuses observations sur ces animaux à l'état de vie, nous avons dû leur affecter. 
I $^{\text {ex }}$ S.-G. Aplysies proprement dites, Rang. Laplysie, Lin.; Dolabelle, Lam.; Actéon, Ocken.

Anim. muni d'une fente dorsale, toujours médiane longitudinale; le pied large, les branchies renfermées dans le fond d'une cavité, d'où leur longueur ne leur permet pas de se porter en dehors, et protégées en dessus par un opercule. Coq. rudimentaire, calcaire, membraneuse, cachée dans l'épaisseur de l'opercule.

$$
1^{\mathrm{er}} \text { groupe. }
$$

Corps renflé en arrière; un disque oblique postérieur;

les bords du manteau serrés sur le dos, et impropres à la natation.

Coquille triangulaire et très calcaire.

Ce groupe comprend le genre Dolabelle de Lamarck. A. rumphii, etc.

$$
2^{\text {e }} \text { groupe. }
$$

Corps rétréci aux deux extrémités; point de disque; les bords du manteau très petits, et impropres à la natation.

Coquille subquadrangulaire et calcaire.

Ce groupe se compose d'espèces nouvelles, à l'exception d'une seule, qui était à tort parmi les Dolabelles.

A. dolabrifera, etc.

\section{$3^{\mathrm{e}}$ groupe.}

Corps rétréci aux deux extrémités; bords du manteau dilatés et propres à la natation.

Coquille subarrondie, membraneuse, et solidifiée par une conche calcaire.

Ce groupe a pour type le genre Laplysie de Linné. Nous le divisons en deux sections.

A. Un tube à la membrane de l'opercule.

A. fasciata, etc. 
MÉTHODIQUE, etc.

B. Une ouverture à la membrane de l'opercule.

A. depilans, etc.

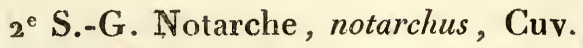

Anim. muni $d^{2}$ une fente dorsale très petite et quel quefois oblique; le pied allongé et assez étroit; les branchies souvent fort longues, et pouvant se porter au-dehors de la cavité; l'opercule rudimentaire ou nul.

Coq. nulle.

Ce sous-genre se compose presque en entier d'espèces nouvelles, et a pour type l'animal dont M. Cuvier avait fait son genre Notarche.

\section{G. GX. Bursatelee, bursatella, Blainv.}

Anim. subglobuleux, offrant inférieurement un espace ovalaire, circonscrit par des lèvres épaisses indiquant le pied; supérieurement, une fente ovalaire, à bords épais, symétrique, formée par la réunion complète des appendices natatoires du manteau, et communiquant dans une cavité où se trouve une très grande branchie libre, et l'anus; quatre tentacules fendus, ramifiés, outre deux appendices buccaux.

Coq. nulle.

Le genre Bursatelle paraît devoir rentrer dans le genre Aplysie où il trouverait sa place dans le second sous-genre à còté des $A$. savignana et pleii. Si nous le reproduisons ici c'est à cause de nos doutes, et, comme nous l'avons fait dans notre Monographie de la famille des $\Lambda$ plysiens, pour le mettre plus en évidence, et provoquer de cette manière l'attention des voyageurs.

Une seule espèce est décrite, c'est la $B$. leachii.

B. Deux tentacules. 


\section{G. CXI. Actéon, actaon, Ocken.}

Elysia P Risso.

Anim. allongé, limaciforme, acuminé postérieurement; manteau muni de deux expansions membraneuses, latérales et propres à la natation; deux tentacules cylindriques, obtus et assez gros; les yeux situés à leur base et en arrière; branchies et organes de la génération inconnus.

Coq. nulle.

Le genre Actéon a été établi par Ocken pour un petit mollusque des côtes du Devonshire décrit par Montagu, mais qui laisse encore infiniment à désirer sous le rapport des caractères extérieurs. Le genre F́lysie de M. Risso ne paraît pas en différer, si l'ou met de côté quelques caractères trop extraordinaires peut-être pour qu'on puisse $\mathrm{y}$ ajouter une grande foi.

\section{$2^{\mathrm{C}}$ FAMILLE.}

LES ACÈRES, Cuv.

Bulléens, Lam.; Acérés, Lat.

Anim. divisé en lobes ou parties distinctes, dont les latéraux se dilatent quelquefois en nageoires; point de tentacules, ou du moins les tentacules formant par leur réunion un disque tentaculaire antérieur; branchies sur le dos, un peu en arrière et à droite, protégées parle manteau et souvent par une coquille. Coq. nulle, interne ou externe.

\section{G. CXII. Acère, akera, Cuv.} Doridium, Meck.; Lobaria, Mül.

Anim. oblong, déprimé, divisé en quatre lobes, l'antérieur ou disque tentaculaire, le postérieur, qui contient les viscères, et les deux latéraux qui sont des expansions amincies du pied, pouvant servir à la natation, et s'abaisser à volonté ou se replier sur le dos; tête non distincte; pied 
très large se continuant avec la surface inférieure des expansions latérales; branchies en forme de panache triangulaire, feuilleté, situé en arrière; anus au côté droit, en arrière et sous l'abri du manteau; organes de la génération très distans, du même côté, l'orifice de la vulve étant situé presqu'en arrière, et l'organe mâle en avant. Coq. nulle.

Une seule espèce, l'A. carnosa, de la Méditerranée a servi à l'établissement de ce genre dont M. Cuvier a fait connaître l'organisation; nous en avons observé plusieurs individus très bien conservés, et qui n'offraient aucune trace de coquille. On a pensé que la 'T. riptère de MM. Quoy et Graimard pouvait se rapporter aux Acères; mais c'est une erreur, car elle appartient évidemment au genre Cléodore.

\section{G. CXIII. Bulle, bulla, Lin. Bulle et Bullée, Lam.}

Anim. oblong, bombé, obtus aux deux extrémités, divisé en quatre lobes, l'antérieur ou disque tentaculaire, le postérieur, qui renferme les viscères, et est recouvert par la coquille, et les deux latéraux en forme de bourrelets, quelquefois très étendus dans le plan du pied, pouvant servir alors à la natation; Ia tête non distincte; le pied grand; les branchies en forme de panache triangulaire feuilleté, situées sur le dos, un peu à droite et en arrière, sous l'abri d'une coquille; anus à l'extrémité d'un petit tube, au côté droit, et un peu en arrière; organes de la génération assez distans, au même côté, l'orifice de la vulve ćtant à la moitié de la longueur, et l'organe mâle tout-à-fait en avant. Coq. ovale, globuleuse, généralement mince et fragile, souvent épidermée, plus ou moins complétement enroulée, à spire ombiliquée ou peu saillante; l'ouverture très grande, de toute sa longueur; le bond droit tranchant. 
Le genre Bulle renferme plusieurs espèces de mollusques dont les coquilles vivantes ou fossiles sont recherchées dans les collections. Lamarck en formait deux genres, les Bulles et les Bullées; les premières ayant une coquille externe et les secondes l'ayant interne; cette distinction n'étant point du tout générique, nous formons, comme M. Cuvier semble l'avoir indiqué, deux sous-genres caractérisés d'après cette même différence qui, du reste, est de bien peu d'importance.

$1^{\mathrm{er}}$ S.-G. Bulles proprement dites.

Une coquille extérieure, quelquefois assez grande pour donner retraite à l'animal. (I)

$$
2 \circ \text { S.-G.Bullées。 }
$$

Une coquille interne, cachée dans l'épaisseur du manteau, ne protégeant qu'une partie de l'animal.

G. CXIV. Gastéroptère, gasteropteron, Meck。 G. Parthenopia, Ock.; Clio amati, Delle Chiaje.

Anim. formé de trois parties : l'antérieure ou disque tentaculaire, la postérieure ou abdominale, qui est oblongue, bursiforme et indépendante du pied, et la troisième, qui est l'organe locomoteur ; celui-ci composé d'un pied adhérent au corps, seulement en avant, étroit, prolongé en arrière et dilaté latéralement en deux vastes nageoires irrégulièrement arrondies; tête non distincte; bouche terminale, deux yeux sur le disque tentaculaire; branchies à nu, sur le côté droit du corps; anus en arrière et au-dessus des branchies; organes de la génération assez rapprochés, l'orifice de la verge étant situé tout-à-fait en avant, et celui de la vulve, à la naissance du peigne branchial; le conduit des œufs en

(x) Nous venons d'en découvrir une nouvelle espèce à Bourbon, qui vit sur les rochers tout près du bord de la mer, mais à l'air libre. 
forme de tube très allongé, pendant au-dessus de l'anus.

Coq. nulle.

Le Gastéroptère est un mollusque très intéressant, que quelques auteurs ont à tort classé parmi les Ptéropodes; M. de Blainville, le premier, a fait connaître sa véritable place. On doit à M. Delle Chiaje, savant anatomiste de Naples, une connaissance approfondie de son organisation interne.

G. CXV. Sormet, sormetus, Adanson.

Anim. allongé, arrondi à chaque extrémité, bombé en dessus, plat en dessous, de largeur égale partout, muni dans toute sa longueur de deux sillons latéraux très profonds; tête non distincte, sans apparence de tentacules; bouche arrondie; branchies communiquant avec le fluide ambiant, par une petite ouverture située au côté droit, qui comprend aussi l'orifice du rectum.

Coq. ovale, déprimée, cornée? à sommet à peine dis tinct et à bords repliés en dedans.

Le genre Sormet, qu'Adanson seul a fait connaître, est trop imparfaitement caractérisé pour que nous ne conservions pas quelques doutes en le mettant dans les Acères; la difficulté de lui trouver une place plus convenable nous a décidé, à l'exemple de MiM. Cuvier et de Blainville, à le laisser ici en attendant de meilleurs renseignemens.

Une seule espèce, le $S$. Adansonii, a servi à l'établissement de ce genre; elle est du Sénégal où Adanson l'a trouvée dans le mois de juin, sur les rives du fleuve, près de son embouchure, enfoncée d'un à deux ponces dans les sables. Ce mollusque établirait assez bien le passage aux Pulmonés.

$$
5^{\mathrm{e}} \text { OKDRE. }
$$

\section{PULMONES INOPERCULÉS, Fér.}

\section{Pulmobranches, Blainv.}

Anim. muni d'un pied pour ramper, point de branchies, mais une cavité pulmonaire recevan! le fluide 
ambiant par une ouverture particulière pratiquée au bord droit du manteau ; les organes de la génération sur le même individu, réunis dans une seule cavité, ou distans.

Coq. nulle, rudimentaire ou complète, interne ou externe.

Les animaux qui ont été rassemblés sous la dénomination de Pulmonés inoperculés diffèrent particulièrement des autres mollusques par la disposition de leurs organes respiratoires; ils ne respirent, en effet, que l'air élastique au moyen d'une cavité toute tapissée à sa paroi supérieure d'un réseau vasculaire. Les Pulmonés inoperculés qui comprennent un assez grand nombre de genres, sont extrêmement répandus; on en trouve sur la terre, dans les eaux douces, et même à l'embouchure des fleuves. C'est parmi eux que l'on remarque des espèces que l'on peut regarder comme domestiques, car elles recherchent sans cesse les lieux que l'homme habite. Ceux de ces animaux qui vivent dans les eaux, sont obligés de venir fréquemment à leur surface pour respirer l'air libre, aussi ne les trouve-t. on ordinairement que dans des eaux très peu profondes. Le travail que M. de Férussac a fait sur les trois premières familles de cet ordre, va nous servir de guide, comme étant ce qu'il y a de plus approfondi sur cette matière; nous n'y ferons que de légers changemens qui nous ont été suggérés par les travaux des naturalistes qui ont écrit depuis lui, et par nos propres observations.

\section{Ire FAMILLE}

\section{LES LIMACES, Fér.}

Limaciens, Lam.; Limacinés, Blainv.; Nudilimaces, Lat.

Anim. allongé, oblong, demi-cylindrique, droit, sans tortillon; manteau presque toujours muni d'une cuirasse charnue, partielle ou générale, débordant quelquefois; tentacules au nombre de quatre, ou de deux seulement, mais alors accompagnés d'une paire d'appendices labiaux; les yeux placés à l'ex- 
trémité des supéríeurs ( $\mathrm{s}$; carité pulmonaire diversement située, mais toujours sous la cuirasse ou sous un test protecteur; orifice de l'anus variable dans sa position; organes de la génération réunis dans une même cavité ouverte derrière le tentacule droit, ou sous l'orifice pulmonaire, quelquefois séparés et distans.

Coq. plus ou moins rudimentaire, ou nulle; interne ou externe; quelquefois remplacée par de petites concrétions calcaires.

Terrestres ou marines.

$\dagger$ Une cuirasse générale.

G. GXVI. Onchune, onchis, Fér. Onchidie, Cuv.; Péronie, Blainv.

Anim. elliptique, convexe en dessus, plat en dessous, muni d'une cuirasse recouvrant tout le corps et dépassant le pied de toutes parts; la bouche située en dessous, entourée d'un bourrelet charnu, et privée de mâchoire; la tête un peu aplatie, munie en avant de deux appendices charnus plus ou moins dilatés; deux tentacules rétractiles, les yeux? la cavité pulmonaire occupant la partie postérieure de l'animal, sous la cuirasse, et s'ouvrant par un orifice arrondi et médian tout-à-fait en arrière, sous le rebord du manteau; l'anus médian situé en avant de l'orifice pulmonaire, les orifices de la génération très distans, celui des coufs s'ouvrant à l'extrémité postérieure du côté droit, et se prolongeant par un sillon latéral jusqu'auprès de l'appendice labial, du même côté; l'orif́ce de l'organe excitateur fort grand, auprès de la base du tentacule droit.

Ce genre comprend les Onchidies marines de M. Cuvier; que M. de Blainville a aussi séparées sous le nom de Péro-

(1) Nous ignorons leur position dans l'Ouchide. 
nies. Avant cela $M$. de Férassac les avait comprises dans ses tableaux systématiques sous celui d'Onchides.

\section{G. CXVII. Oлchide, onchidium, Buchanan Véronicelle, Blainv.; Vaginule, Fér.}

Anim. oblong, allongé, souvent effilé dans l'état d'extension, convexe en dessus; une cuirasse recouvrant tout le corps, le débordant, formant en avant une sorte de capuchon où la tête peut se retirer; bouche armée d'une mâchoire supérieure; quatre tentacules contractiles, les deux supérieurs longs et oculifères, les antérieurs courts et comme palmés ou bifurqués à leur extrémité; le pied oblong, allongé; la cavité pulmonaire vers le milieu du corps, ayant son orifice en arrière, à l'extrémité d'un long canal, et séparé de l'anus par une seule membrane; organes de la génération très distans, à droite, l'organe mâle étant près du petit tentacule, et l'orifice des cufs vers le milieu; point de pore muqueux terminal.

Coq., ni test rudimentaire interne, ni concrétion calcaire.

M. de Férussac a établi le genre Vaginule pour des mollusques qui ne paraissent différer en rien d'essentiel des Onchidies; M. de Blainville a le premier signalé le rapprochement nécessaire de ces deux genres, et nous l'adoptons d'autant plus volontiers que nous avons pu nous convaincre sur les $V$.punctulatus et krausiï que nous avons vues vivantes, et que nous avons communiquées à $M$. de Férussac, que les animaux de ce dernier genre se rapportent parfaitement à la caractéristique des Onchidies. A ces dernières se trouve encore réuni le genre Véronicelle proposé par M. de Blainville, et adopté par M. de Férussac, mais que le premier de ces savans, d'après de nouvelles considérations, a jugé plus convenable de faire disparaître. Les Onchidies se composent maintenant de plusieurs espèces; mais quelques unes d'elles sout très mal connues. On les dit terrestres et d'eaux 
douces; nous ne les avons jamais rencontrèes à Bourbon et à la Martinique que dans les bois et les jardins, sous les vieux troncs renversés.

Après le genre que nous venons de décrire, M. de Férussac en signale deux autres dus à M. Rafinesque, les Phylomiques et les Eumèles; mais leurs caractères sont présentés d'une manière si insuffisante, et quelques uns d'eux paraissent si singuliers que nous n'en tiendrons pas compte, aimant mieux attendre de nouveaux documens à leur sujet.

†† Une cuirasse partielle.

G. CXVIII. Lrmacelle, limacella, Blainv.

Anim. allongé, subcylindrique, pourvu d'un pied aussi long et aussi large que lui, dont il n'est séparé que par un sillon; enveloppé dans une peau épaisse, formant à la partie antérieure du dos une sorte de bouclier protecteur de la cavité pulmonaire, dont l'orifice est à son bord droit; les orifices de l'appareil générateur distans, celui de l'oviducte à la partie postérieure, du côté droit, et communiquant par un sillon avec la terminaison de l'organe mâle, située à la racine du tentacule droit.

L'auteur de ce genre, après l'avoir décrit comme on vient de le voir, ajoute: "Cette combinaison de caractères nous paraît si anomale, que nous doutons réellement que nous ayons bien observé le mollusque sur lequel nous avous établi ce genre. »

\section{G. CXIX. Limace, limax, Lam. Et Arion, Fér.}

Anim. oblong plus ou moins allongé, demi-cylindrique, muni d'une cuirasse à la partie antérieure; tête assez distincte, rétractile sous la cuirasse, portant deux paires de tentacules également rétractiles, terminés en bonton, les supérieurs longs et oculifères, les inférieurs courts; pied grand et oblong, cavité pulmonaire située sous la cuirasse, 
et souvrant sous son bord droit; orifice de l'anus au bord postérieur de celui de la cavité respiratrice; organes de la génération réunis et montrant le leur au côté droit antérieur, près du grand tentacule; quelquefois un pore muqueux terminal. Coq. Un test rudimentaire interne, ou des concrétions calcaires dans l'épaisseur de la cuirasse.

Les Limaces sont des mollusques terrestres très communs et connus de tout le monde; on les rencontre partout, mais particulièrement sur les bords des fossés et dans les jardins potagers où elles causent parfois de grands dommages. Les contrées septentrionales paraissent en avoir un plus grand nombre que celles de la zone torride. Il en existe en Afrique, à la Nouvelle-Hollande, et nous en avons vu dans l'Inde et à l'Ile-de-France.

M. de Férussac a saisi quelques anomalies dans les caractì̀res de ces mollusques qui lui ont donné lieu d'en séparer un certain nombre dont il a fait le genre Arion. Cette distinction n'a été adoptée par M. de Blainville que pour l'établissement de deux sections; nous pensons qu'il convient mienx d'en faire deux sous - genres dout la caractéristique serait ainsi.

$$
\text { I }^{\text {ex }} \text { S.-G. Arion, Fér. }
$$

Orifice pulmonaire plus en avant; cuirasse chagrinée, contenant de petites concrétions calcaires; un pore muqueux terminal.

$2^{\mathrm{e}}$ S.-G. Limaces proprement dites.

Orifice pulmonaire plus en arrière; cuirasse marquée de stries fines et concentriques, contenant un rudiment testacé, solide, sans aucune empreinte volutatoire; point de pore muqueux.

\section{G. GXX. Parmacelle, parmacellus, Guv.}

Anim. allongé, oblong, demi-cylindrique, recouvert sur le milieu du dos d'une cuirasse arrondie, 
oblongue, charnue, et en grande partie libre en avant; tête assez distincte, portant deux paires de tentacules rétractiles, l'une supérieure, longue et oculifère, l'autre antérieure et courte; pied grand et oblong; cavité pulmonaire sous la partie postérieure de la cuirasse, s'ouvrant, ainsi que l'anus, par une solution de continuité commune, sous son bord droit, un peu en arrière; orifice de la génération, unique, près du tentacule droit.

Coq. aplatie, calcaire, avec un épiderme membraneux, ovale, légèrement courbe dans le sens de la largeur, à sommet marqué par un sinus profond au côté droit postérieur; placée dans l'épaisseur de la cuirasse, au-dessus de la cavité pulmonaire.

Les Parmacelles forment un genre bien naturel et très voisin des limaces; elles habitent dans les bois au Brésil; mais à Bourbon et à Madagascar nous ne les avons trouvées que sur des rochers, au bord des torrens d'eau douce. Olivier en a le premier rapporté une de la Mésopotamie. C'est celle qui a servi aux recherches anatomiques de $\mathrm{M}$. $\mathrm{Cu}$ vier sous le nom de $P$. olivieri; depuis M. de Férussac en a décrit une autre sous le nom de $P$. pailiolum. Enfin nous en avons rapporté de notre voyage dans les mers de l'Inde, deux autres, dont l'une, $P$. Rangianus, a été décrite par M. de Férussac comme étant un Arion (Bull. des Sciences, février 1827); elle est des îles de Bourbon et de Madagascar.

†† Point de cuirasse.

\section{G. CXXI. Testacelle, testacellus, Cuv。 Et Plectrophore, Fér.}

Anim. allongé, cylindriforme, acuminé à chaque extrémité ; cuirasse nulle; tête assez distincte, munie de quatre tentacules rétractiles, dont les postérieurs, qui sont les plus longs, portent les yeux; pied long et peu distinct ; cavité pulmonaire située au quart postérieur de la longueur, son orifice tout-à-fait 
en arrière, sous le côté droit du sommet de la coquille, celui de l'anus en est très voisin; les organes de la génération réunis, et montrant leur orifice près et en arrière du grand tentacule droit.

Coq. extérieure, solide, auriforme, déprimée, à spire plus ou moins saillante, ayant une ouverture très grande et ovale; le bord droit simple et tranchant, le gauche renflé et réfléchi; elle recouvre la partie postérieure de la cavité pulmonaire.

M. de Férussac a remarqué que ce mollusque était muni d'un manteau « simple, gélatineux, contractile, caché habituellement sous le test, divisé en plusieurs lobes susceptibles d'envelopper tout le corps par un développement extraordinaire, lorsque l'animal éprouve le besoin de se garantir de la sécheresse. » Une espèce de Ténériffe, T. maugei, très distincte de celle d'Europe, a été acclimatée dans le jardin botanique de Bristol, où elle a singulièrement multiplié depuis quelques années.

On rapportait au genre Testacelle quelques mollusques mal connus, que M. de Férussac a séparés pour en former le genre Plectrophore. Nous ne croyons pas devoir tenir compte de cette distinction générique à cause du peu de solidité des caractères sur laquelle elle est établie.

\section{$2^{e}$ FAMICLE.}

LES LIMAÇONS, Fér.

Trachélipodes colimacés, Lam.; Limacinés, Blainv.; Géocochlides, Lat.

Anim. allongé, ayant le corps distinct du pied, et formant un tortillon contourné en spirale, rarement muni d'une cuirasse, mais montrant toujours un collier charnu qui ferme la coquille; tentacules au nombre de quatre, rarement de deux, les supérieurs oculés; cavité pulmonaire en avant, s'ouvrant dans l'épaisseur du collier; organes de la génération réunis en avant; anus près de l'orifice respiratoire. 
Cog. Toujours une coquille spirale, de forme très variable, recevant plus ou moins complétement l'animal.

Terrestres.

\section{† Tétracères.}

A. Une cuirasse et un collier.

\section{G. CXXII. Vitrine, vitrina, Draparnaud. Hélicolimace et Hélicarion, Fér.}

Anim. allongé, demi-cylindrique, ayant un petit tortillon, un collier charnu cernant le cou et fournissant en avant une sorte d'appendice qui s'étend sur lui en forme de cuirasse, et quelques autres appendices linguiformes rétractiles, capables de recouvrir presque toute la coquille; tentacules au nombre de quatre, cylindriques et rétractiles, les deux supérieurs oculés au sommet; pied séparé du corps par un petit sillon; orifice de la cavité pulmonaire à droite, sur le collier, à la naissance de la cuirasse; organes de la génération réunis et présentant leur orifice près du tentacule droit; quelquefois un pore muqueux à la partie postérieure. Coq très petite, spirale, mince, transparente et fragile, croissant rapidement dans le sens horizontal; spire courte, le dernier tour très grand; ouverture vaste, avec une columelle solide, spirale, se confondant presque toujours avec le tour de l'ou verture.

M. de Férussac, à qui la science doit la connaissance de plusieurs espèces de ce genre, le décrit sous le nom d'hélicolimace; il en distingue quelques espèces exotiques qui lui ont paru se rapprocher un peu plus des Parmacelles, et dont il fait son genre Hélicarion. Les différences génériques sur lesquelles sont établis les Hélicolimaces et les Hélicarions ne nous paraissant pas justifiées par des caractères suffisans, nous nous en tenons jusqu'à nouvel ordre au genre Vitrine, tel qu'il a été établi par Draparnaud. 
Ces animaux habitent sur les plantes, sous les feuilles mortes et dans les creux des rochers.

B. Un collier sans cuirasse.

G. CXXIII. HéLIGE, helix, Muller.

Hélice, Succinée, Amphibulime, Acave, Polydonte, To- mogère, Anostome, Caracole, Bulime, Achatine, Poly(I phème, Maflots, Clausilie, etc., etc., Fér.

Anim. de forme peu variable, demi-cylindrique, muni d'un tortillon très grand; collier charnu, fermant exactement la coquille, et portant quelques appendices courts; tête assez distincte; bouche fendue en long, pourvue de chaque côté d'un lobe charnu, et en dedans d'une masse linguale et d'une pièce supérieure, dentée et propre à la mastication; quatre tentacules rétractiles, renflés en bouton à leur sommet, les supérieurs étant les plus longs et oculés; pied grand et oblong, allongé ; orifice de la cavité pulmonaire à droite, sur le collier; anus tout à côté et un peu plus à droite; organes de la génération réunis, et ayant leur orifice près du tentacule droit postérieur.

Coq. très variable dans sa forme, planorbique, globuleuse, ovoïde, conoïde, fusiforme on turriculée; à volute croissait plus ou moins rapidement; variant dans la forme de son ouverture et dans son plan, par rapport à l'axe; à ouverture simple, dentée ou garnie de lames; un ombilic visible ou couvert; trois à quatorze tours de spire; elles sont généralement dextres et quelquefois sénestres.

Le genre Hélice, le plus généralement répandu dans les cinq parties du monde, est celui qui fournit jusqu'ici le plus grand nombre d'espèces; on en rencontre partout, excepté dans les eaux. La science doit à M. le baron de Férussac un beau travail à leur sujet, ainsi que la connaissance d'un nombre très considérable d'espèces nouvelles, et que plusieurs amatẹurs zélés de l'histoire naturclle se sont em- 
presisés de recueillir dans les diverses contrées du globe, afin de le seconder dans une si belle entreprise. Une exposition succincte de ce travail, que le prix élevé des magnifiques planches dont il est accompagné ne met qu'à la portée d'un petit nombre de personnes, va faire connaître la richesse du genre et la classification méthodique que ce savant a dû adopter parmi les espèces, afin d'en rendre la détermination plus facile.

Les animaux des Hélices ne diffèrent que faiblement entre eux, ce n'est donc que d'après la considération des caractères de la coquille que cette classification a pu être établie.

Ces coquilles sont fort recherchées dans les cabinets, surtout depuis que M. de Férussac y a fixé l'attention des conchyliologistes.

(†) Redundantes.

† Volutato. Héurcö̈ns, helicoides.

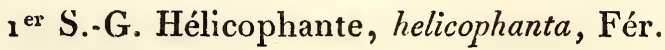

Coq. très petite pour l'animal, croissant rapidement dans le sens horizontal, à spire peu saillante, à ouverture très ample, oblique, perforée ou ombiliquée.

\section{Péristome simple.}

I er groupe. Les Vitrinoïdes, vitrinoides, F.

H. brevipes, etc.

Péristome épaissi et subréfléchi.

$2^{\mathrm{e}}$ groupe. Les Vessies, vesicula, $\mathbf{F}$.

H. cafra, etc.

†卜 Evolutata. Cochroïns, cochloides.

$2^{\ominus}$ S.-G. Coclohydre, coclohydra, Fér. Succinea, Drap.; Amphibulima, Lam.

Coq. trop petite pour l'animal, allongée ou ovale; volute rapidement développée dans le sens vertical; spire courte, le dernier tour formant presque toute la coquille; ouverture grande. 
Elles recherchent souvent le bord des eaux douces.

(†) Inclusa.

i. Volutata. Héxrcoides, helicoides.

3 S.-G. Hélicogène, helicogena, Fér. Helix, Lin.

Coq. globuleuse ou surbaissée; spire courte, dernier tour beaucoup plus renflé que les précédens réunis, et composant presque toute la coquille; généralement un vide ombilical un peu spiral ou cylindrique, ombilic masqué ou couvert; bouche régulière, semi-lunaire, sans dents ; péristome épaissi ou réfléchi, mais non bordé.

\section{Columelle solide et torse.}

I $^{\text {er }}$ groupe. Les Columellées, columellata, F.

a) Péristome simple.

H. naticoides, etc.

6) Péristome réfléchi ou épaissi.

H. jamaicensis, etc.

\section{Coquille perforée.}

$2^{\mathrm{e}}$ groupe. Les Perforées, perforata, F.

II. ligata, etc.

Coquille ombiliquée: ombilic tout couvert.

a) Coquille globuleuse ou subtrochoïde.

$$
\begin{gathered}
3^{e} \text { groupe. Les Acaves, acavee. } \\
\text { G. Acave, Montf. }
\end{gathered}
$$

H. aspersa, etc.

Coquille imperforée.

k) Coquille surbaissée. 
$4^{\circ}$ groupe. Les Imperforées, imperforata, F.

x) Bouche arrondie, péristome évasé.

H. guttata, etc.

2) Bouche sinueuse, péristome fortement réfléchi.

H. squamosa, etc.

3) Bouche versante, bord columellaire, sinueux, aplati et subdenté.

H. cognata, etc.

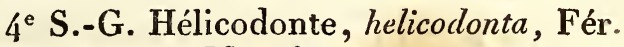

Tomogère, Montf.; Anostome, Lam.

Coq. globuleuse ou déprimée; volute lentement développée, impulsion horizontale dominante; spire courte, à tours égalisés ; bord intérieur portant généralement sur l'avant-dernier tour; ombilic visible ou masqué; bouche sinueuse, grimaçante, et généralement dentée dans l'état parfait; péristome réfléchi ou épaissi.

Péristome sinueux et épais, ou réfléchi et denté, souvent rétréci par les dents, les lames ou les plis tortueux de la convexité de l'avant-dernier tour.

$\mathrm{I}^{\mathrm{er}}$ groupe. Les Grimaces, personate, $\mathrm{F}$.

$H$, dentiens, etc.

Ouverture défendue par une ou plusieurs lames allongées ê internes.

$2^{\mathrm{e}}$ groupe. Les Lamellées, lamellata, F.

* Plusieurs lames (planiformes).

H. carabinata, etc.

** Une seule lame.

H. labyrinthica, etc.

Péristome garni de grosses dents, dont l'une a la base de la columelle formant gouttière. 
3e groupe. Les Maxillées, maxillatae.

G. Polydonte, Montf.

H. imperator, etc.

Bouche renversée, garnic de plis élevés, dont les impressions sont visibles au-dehors.

$4^{\mathrm{e}}$ groupe. Les Anostomes, anostoma, $\mathbf{F}$.

$H$. ringens, etc.

Bord intérieur de l'ouverture, garni près du péristome de plis longitudinaux élevés, dont les impressions sont visibles au-dehors.

$5^{\text {e }}$ groupe. Les Impressionées, impressa.

H. cepa, etc.

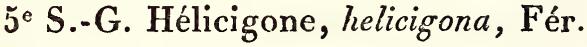

Coq. lenticulaire ou coniforme, plus ou moins carénée; volute le plus souvent courte, quelquefois conique; tours de spire en général égalisés; columelle rarement solide; bord intérieur du cône spiral portant presque toujours sur la convexité de l'avant-dernier tour; ombilic couvert ou visible; bouche sans dents, versante, anguleuse à la réunion des deux bords opposés, où l'impression carénale forme une sorte de gouttière; péristome épaissi ou réfléchi, mais non bordé.

Ombilic couvere.

I $^{\mathrm{er}}$ groupe. Les Caracolles, caracollo, Montf.

$H$. angistoma, etc.

Ombilic masqué ou visible.

$2^{\mathrm{e}}$ groupe. Les Tourbillons, vortices, Ock.

H. marginala, etc. 


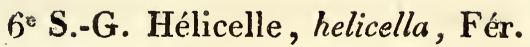

Coq. généralement surbaissée ou déprimée; volute peu élevée, souvent planiforme; tours de spire communément égalisés et arrondis; bord intérieur du cône spiral portant sur la convexité de l'avant-dernier tour; ombilic découvert, plus ou moins large ou étroit, et laissant généralement apercevoir jusqu'au sommet de la spire; bouche sans dents; péristome réfléchi, simple ou bordé; quelquefois la coquille est carénée ou imperforée, mais alors le péristome est bordé.

Péristome réfléchi.

$\mathrm{I}^{\mathrm{er}}$ groupe. Les Lomastomes, lomastoma, F.

H. carascalensis, etc.

Péristome simple.

$2^{\text {e }}$ groupe. Les Aplostomes, aplostoma, $\mathbf{F}$.

* Les Pesons, verticilli.

$H$. lineata, etc.

** Les Hyalines, hyalina.

$H$. olivetorum, etc.

*** Les Rubannées, fasciatoe.

H. candida, etc.

Péristome bordé.

a) Coquille couleur de corne ou brune, presque unicolore, rarement fasciée, souvent velue; péristome un peu évasé; épiderme caduc.

$3^{\mathrm{e}}$ groupe. Les Hygromanes, hygromanes, F. H. cinctella, etc.

B) Coquille blanche ou rousse, très omée de fascies ou de linéoles de couleurs vives; épiderme insensible, jamais velu; quelquefois carénée; péristome bordé, mais nore évasé. 
$4^{\text {e }}$ groupe. Les Héliomanes, heliomanes, $F$.

* Coquille surbaissée ou globuleuse.

$H$. groyana, etc.

** Coquille trochoïde et un peu carénée.

H. pyramidata, ete.

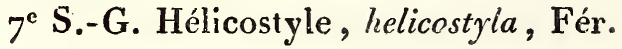

Coq. surbaissée ou trachiforme; volute quelquefois allongée; tours de spire communément égalisés, pressés, étroits; bord intérieur du cône spiral, formant une columelle solide, droite ou torse ; aire ombilicale souvent enfoncée; bouche régulière, plus ou moins oblique à l'axe du test; péristome simple ou réfléchi.

Columelle droite, péristome simple; coquille surbaissée.

I $^{\mathrm{er}}$ groupe. Les Aplostomes, aplostoma, F.

H. misella, etc

Columelle torse, comme tronquée à sa base, ou munie d'une côte spirale interne, formant gouttière, et paraissunt sous la forme d'une dent ou d'une callosité.

$2^{e}$ groupe. Les Canaliculées, canaliculata, $\mathrm{F}$.

H. delicatula, etc.

Columelle aplatie, sans dent ni lame, formant une sorte de gouttière à son intersection avec l'avant-dernier tour; péristome réfléchi.

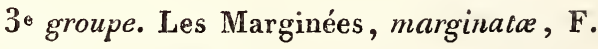

H. stsderiana, etc.

$\dagger \dagger$ Evolutata. Cochrö̈nes, cochloides.

(*) Bouche généralement sans dent.

I) Une columelle solide.

a) En filet, non tronquée ì sa base. 
8e S-G. Cochlostyle, cochlostyla, Fér.

Coq. allongée et ventrue; volute développée dans le sens vertical; spire élevée, tours croissant fortement; bord intérieur du cône spiral, formant une columelle solide, torse, non tronquée; bouche plus ou moins verticale par rapport à l'axe; bord extérieur plus ou moins avancé; péristome simple ou réfléchi.

Péristome réfléchi.

$I^{\mathrm{er}}$ groupe. Les Lomastomes, lomastoma, F.

H. metaformis, etc.

\section{Péristome simple.}

$2^{\text {e }}$ groupe. Les Aplostomes, aplostoma, F.

H. dufresnii, etc.

6) Columelle solide, aplatie et tronquée à sa base.

† Coquille conique ou très ventrue; ouverture élargie.

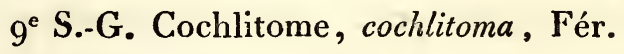

Coq. conique ou très ventrue, solide, peu transparente; volute croissant plus ou moins fortement; spire plus ou moins élevée; bord intérieur du cône spiral, formant une columelle plate, torse, solide, repliée en dedans, et plus ou moins tronquée à sa base; ouverture plus ou moins courte ou longue, et droite, c'est-à-dire dans la direction de l'axe, mais élargie; bord extérieur plus ou moins dans la verticale; péristome simple.

Coquille conique, bouche courte, bord extérieur avancé.

$$
{ }^{\text {er }} \text { groupe. Les Rubans, liguuce, F. }
$$

H. exarata, etc.

Coquille ventrue, bouche très grande, bord extérieur dans la verticale. 
$2^{\circ}$ groupe. Les Agathines, achatina, F.

H. fulvescens, etc.

† Coquille ovoïde ou turriculée, bouche allongée et étroite.

$10^{\mathrm{e}}$ S.-G. Cochlicope, cochlicopa, Fér.

Coq. ovoïde ou turriculée, mince, transparente, généralement unicolore; volute croissant plus ou moins fortement; spire courte ou très allongée; bord intérieur du cône spiral, formant une columelle plate, torse, solide, repliée en dedans, et plus ou moins arquée et tronquée à sa base; ouverture plus ou moins courte ou allongée, et droite, mais généralement étroite; péristome simple.

Coquille ovoïde, bouche longue, bord extérieur dans la verticale.

I $^{\text {er }}$ groupe. Les Polyphêmes, polyphema, Montf.

H. priamus, etc.

Coquille turriculée, bouche courte, bord extérieur un peu avancé.

$2^{\circ}$ groupe, Les Styloïdes, styloides, F.

H. fulminea, etc.

2) Coquille perforée ou ombiliquée; ombilic masqué ou découvert; péristome simple.

a) Tours de spire égalisés, le dernier moins long que les autres réunis.

$1^{\circ}$ S.-G. Cochricelle, cochlicella, Fér.

Coq. conique ou turricalée, perforée ; tours de spire égalisés, nombreux, étroits, le dernier moins long que les autres réunis; ouverture courte ; $\mathrm{co}$ lumelle torse et creuse.

Groupe unique. Les Tourelles, turrite, F. H. conoidea, etc. 
(6) Dernier tour de spire généralement plus gros et plus long que les autres réunis.

I $2^{\mathrm{e}}$ S.-G. Cochlogìne, cochlogena, Fér.

Coq. oblongue ou ovoïde; dernier tour de spire généralement plus gros et plus long que les autres réunis; bord intérieur du cône spiral replié en dehors, et portant presqu'à angle droit sur la convexité de l'avant-dernier tour, de manière à former une columelle creuse, torse ou droite, perforée ou ombiliquée; ouverture généralement allongée et en croissant.

† Péristome simple ou épaissi, mais $\dot{c}_{\iota}$ bords tranchans.

a) Coquille ombiliquée, columelle droite.

I $^{\text {er }}$ groupe. Les Ombiliquées, umbilicata, F.

H. flammata, etc.

B) Coquille perforée, columelle torse.

$2^{\mathrm{e}}$ groupe. Les Perforées, perforata, $\mathrm{F}$.

* Coquille oblongue.

H. fasciolata, etc.

** Coquilles ovoïdes.

H. costulata, etc.

† Péristome réfléchi ou denté.

Bouche en croissant, sans dents ni plis; péristome réfléchi et régulier; columelle torse, perforée; dernier tour de spire quelquefois plus court que les autres réunis.

$3^{\mathrm{e}}$ groupe. Les Lomastomes, lomastoma, F.

* Dernier tour de spire plus gros et plus long que les autres réunis; coquille ornée de couleurs vives.

$H$. favanii, etc.

** Dernier tour moins long et moins gros que les autres réunis; coquille unicolore.

H. beticata, etc. 
Bouche courte, en croissant; péristome simple ou épaissi et régulier; columelle torse plus ou moins saillante et arquée, ou munie d'un pli qui tourne sur elle, et la fait paraître subtronquée; ombilic niasqué ou exactement clos; dernier tour de spire quelquefois plus court que les autres réunis.

$4^{\mathrm{e}}$ groupe. Les Hélictères, helicteres, $\mathrm{F}$.

* Coq. coniforme.

H. vulpina, etc.

** Coq. turriculée.

H. turritella, etc.

*** Coq. ovoïde.

H. tristis, etc.

Bouche allongée, anguleuse à ses extrémités, ou versante supérieurement, souvent rétrécie par les sinuosités du bord extérieur; columelle grosse, plus ou moius spirale, et formant un pli plus ou moins saillant dans l'ouverture. Péristome épaissi et réfléchi; dernier tour de spire plus long et plus renflé que les autres réunis.

$5^{\mathrm{e}}$ groupe. Les Stomotoïdes, stomotoides, F.

H. auris leporis, etc.

Bouche en croissant, un peu anguleuse à ses extrémités, munie le plus souvent de dents courtes au péristome, qui est bordé ou un peu évasé ou réfléchi; jumais de lames; columelle torse, creuse, aplatie à sa base, ou formant une protubérance saillante; généralement perforée.

$6^{\mathrm{e}}$ groupe. Les Dontostomes, dontostoma.

*) Dernier tour de spire plus gros et plus long que les autres réunis.

H. auris bovinus, etc.

**) Tours de spire égalisés, souvent pressés et étroits.

H. turgens, etc.

$\left(^{* *}\right)$ Bouche généralement garnie de dents ou de lames.

I) Sans gouttières; péristome généralement non continu. 
i 3e S.-G. Cochlovonte, cochlodonta, Fér.

Coq. cylindracée ou fusiforme, tours de spire nombreux, égalisés, pressés, étroits; columelle généralement solide, en filet spiral, rarement creuse, alors conico-cylindrique et ombiliquée; bouche courte, presque aussi large que haute, droite, c'est-à-dire placée dans la direction de l'axe, presque toujours garnie intérieurement de dents allongées ou de lames minces, ses deux bords sur le inême plan vertical, et tombant presqu'à la même hauteur, sur la convexité de l'avantdernier tour; péristome réfléchi, généralement non continu.

\section{Coquille cylindrique.}

I er groupe. Les Maillots, pupa, F.

H. uva, etc.

Coquille fusiforme.

$2^{\mathrm{e}}$ groupe. Les Grenailles, cereales, $\mathrm{F}$.

H. moricandi, etc.

2) Une ou deux gouttières; péristome généralerient continu.

I $4^{e}$ S.-G. Cochlodine, cochlodina, Fér.

Coq. cylindracée ou fusiforme; tours de spire nombreux , pressés, égalisés, étroits; columelle solide, en filet spiral, souvent garnie de lames, tournant avec elle, et d'une sorte d'opercule pédonculé et élastique; bouche garnie le plus souvent de lames élevées, et toujours d'une ou deux gouttières, la supérieure formée par une carène dorsale ; péristome presque toujours continu.

(*) Coquille dextre.

† Bouche sans dents ni lames. 


\section{Péristome non continu.}

$1^{\text {er }}$ groupe. Les Pupoïdes, pupoides, T?.

H. carinula, etc.

Péristome continu.

$\mathbf{2}^{\mathrm{e}}$ groupe. Les Trachéloïdes, tracheloides, F.

H. Sloanii, etc.

† Bouche armée de gros plis ou dents allongées.

H. gargantua.

$\left.{ }^{* *}\right)$ Coquille sénestre.

Bouche sans lame.

3e groupe. Les Anomales, anomales, F.

H. perversa, etc.

Bouche armée (des lames dont une en opercule élastique).

$4^{\mathrm{e}}$ groupe. Les Clausilies, clausilia, Draparnaud.

H. torticollis, etc.

Il existe plusieurs espèces d'hélice à l'état fossile.

䂛 Dicères.

G. CXXIV. Vertigo, vertigo, Muller.

Anim. allongé, demi-cylindrique, ayant un tortillon assez grand et un collier fermant la coquille; deux tentacules seulement, longs, obconiques, rétractiles, arrondis à leur extrémité; l’orifice de la cavité pulmonaire sur le collier et à droite, avoisiné par celui de l'anus; organes de la génération réunis et montrant leur orifice près du tentacule droit. Ovipares.

Coq. cylindrique, très spirale; volute croissant lentement; cône spiral incomplet; ouverture droite, dans la direction de l'axe, courte, souvent dentée; 
pérístome souvent sinueux et réfléchi; dextres ou sénestres.

Le genre Vertigo, dont la distinction générique n'est absolument basée que sur l'absence de la paire de tentacules antérieurs, ne renferme qu'un petit nombre d'espèces toutes vivantes.

G. CXXV. Partule, partula, Fér.

Anim. allongé, demi-cylindrique, avec un tortillon assez grand; un collier fermant la coquille et portant l'orifice de la cavité pulmonaire à droite et à l'angle extérieur de l'ouverture; deux tentacules seulement, cylindriques et rétractiles, oculés à leur sommet; organes de la génération réunis? montrant leur orifice près du tentacule droit. Ovovivipares.

Coq. ovale, pointue; spire conique, dernier tour renflé et plus long que les autres réunis, quatre à six tours de spire; cône spiral incomplet; ouverture droite dans la direction de l'axe, courte, quelquefois dentée ou munie de lames élevées; péristome communément fort réfléchi, à bords dans le même plan vertical; côté columellaire, calleux à sa base; dextres ou sénestres.

Le genre Partule a été établi par M. de Férussac, pour de petits mollusques terrestres qui, outre les différences qu'ils présentent dans l'aspect de leur coquille, comparée à celle des Vertigos, offrent cette particularité remarquable qu'ils sont ovo-vivipares, tandis que les autres sont seulement ovipares. Nous pensons comme ce savant que l'organisation des Partules mérite d'être examinée avec soin, et que l'on y trouvera peut-être occasion de justifier une dis tinction générique qui, à l'extérieur, ne reçoit, il est vrai, aucun appui suffisant, mais qui peut exister dans le système de la génération. Ces considérations nous engagent à adopter jusqu'à nouvel ordre le genre Partule. Les espèces désignées par ce naturaliste sont, en partie, des terres australes. 


\section{3e FAMILIE.}

LES AURICULES, Fér.

Auriculacés, Blainv.; Limnocochlides (à collier), Lat.

Anim. allongé, ayant le corps distinct du pied, et contourné en spirale; jamais de cuirasse, un collier; tentacules au nombre de deux; yeux non pédonculés, à leur base ou près de leur base; bouche généralement armée d'une dent supérieure opposée à une langue à crochets; cavité pulmonaire et son orifice situés en avant; organes de la génération réunis ou distans.

Coq. toujours spirale, assez variable, à ouverture latérale par rapport à l'axe du cône, et dentée. Terrestres ou marins; fluviatiles?

\section{G. CXXVI. Carichie, carychium, Mull.}

Anim. allongé, portant l'orifice de la cavité pulmonaire au cồté droit du collier; tentacules rétractiles, gros, cylindriques et obtus; yeux situés à la base postérieure des tentacules; organes de la génération? anus? pied non divisé.

Coq. allongée, oblongue ou cylindrique; volute croissant assez lentement; cône spiral incomplet; ouverture droite, courte, simple, ou avec des plis ou des dents.

Le genre Carychie et les suivans ne diffèrent entre eux que par leur coquille à peu près, ce qui fait que l'on pourrait les réunir, en leur laissant seulement une distinction de second ordre. Malgré cette observation, nous les laissons tels que M. de Férussac les a présentés dans son travail sur les Pulmonés géhydrophiles, en attendant que l'on ait à leur sujet une connaissance plus étendue. Les différences caractéristiques de leurs coquilles offrent d'ailleurs des moyens de justifier jusqu'à un certain point quelques distinctions parmi eux. 
G. CXXVII. Auricule, auricula, Lam. Conovule, Lam.; et Mélampe, Montf.

Anim. allongé , muni antérieurement d'un mufle plus ou moins proboscidiforme; tentacules contractiles, courts, cylindriques, en forme de gland, au sommet; yeux situés à la base interne des tentacules, un peu en arrière; pied non divisé.

Coq. ovale, plus ou moins pointue et allongée, rarement cylindrique; spire souvent enveloppante, de cinq à six tours contigus, quelquefois peu distincts, le dernier formant presque tout le test; ouverture allongée en forme d'oreille, souvent très étroite; péristome épaissi, bord extérieur simple ou denté; columelle torse, solide, communément sans indice de fente ombilicale, garnie d'une à trois côtes saillantes, tournant avec elle dans l'intérieur.

Le genre Auricule demande à être mieux connu. Nous en avons rencontré au Brésil, et surtout à l'Ile-de-France et à Madagascar, respirant l'air libre sur les rochers au bord de la mer. Jamais nous ne les avons trouvées dans l'eau; mais M. Lesson en annonce une d'ean douce.

M. de Férussac en a formé trois groupes.

$1^{\mathrm{er}}$ groupe. Les Auricules, auricula.

G. Auricule, Lam.

A. midae, etc.

Plusieurs espèces fossiles font partie de ce groupe.

$2^{\mathrm{e}}$ groupe. Les Conovules, conovula, Lam.

A. ovula, etc.

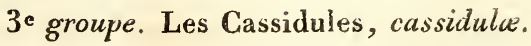

A. cassidula, etc.

G. CXXVIII. Piétin, pedipes, Adans.

Anim. muni de tentacules filiformes, implantés verticalement sur la tête, et divergeant; les yeux ovales 
et stués au-dedans et à la base des tentacules; pied de forme elliptique, partagé en deux talons par un large sillon transversal; bouche munie d'une pièce supérieure correspondant à une masse linguale armée de petits crochets.

Coq. globulaire ou ovale, épaisse, à sommet peu sail. lant; le dernier tour de spire plus grand que les autres réunis; ouverture longue, ovale ou linéaire, à bords non réunis; columelle solide, munie de deux lames saillantes; une troisième fort élevée sur la convexité de l'avant-dernier tour; bord extérieur tranchant, et muni quelquefois en dedans de petites côtes.

Le genre Piétin n'est connu que par la description qu'en a donnée Adanson. Nous avons besoin de nouveaux renseignemens pour être fixé à son sujet. M. de Blainville lui rérnit les Conovules de Lamarck.

\section{G. CXXIX. Scarabe, scarabus, Montf.}

Anim. allongé, comprimé, très spiral, muni de tentacules déprimés, triangulaires, oculés à leur base interne; orifice de la cavité pulmonaire situé à l'angle extérieur du collier, du côté droit, et avoisiré par l'anus; organes de la génération séparés, orifice mâle à droite, en arrière et en dessous de la lèvre, près du pied, orifice des œufs près de la séparation du tortillon, communiquant par un sillon? Coq. ovale, pointue, comprimée dans le sens de sa longueur, et parallélement au plan de l'ouverture, de manière à former deux arêtes latérales; spire enveloppante, de huit à neuf tours contigus, le dernier formant les deux tiers du test; sutures recouvertes; ouverture longue, arquée, étroite, garnie de dents ou de lames sur chaque lèvre; péristome continu et tranchant, le bord intérieur replié vers la base de la columelle, celle-ci garnie de lames élevées tournant avec elle; fossette ombilicale, si- 
nueuse, plus ou moins perforée, quelquefois ombiliquée.

C'est à M. de Férussac, qui le premier a fait connaître l'animal du Scarabe, que nous empruntons les caractères de ce genre. Ce sont réellement des mollusques terrestres, et que les naturalistes de l'expédition de M. le capitaine Freycinet ont même trouvés sur le haut des montagnes.

$4^{\circ}$ FAMILLE.

LES LIMNÉENS, Lam.

Limnacés, Blainv.; Limnocochlides (sans collier), Lat.

Anim. allongé, ayant le corps distinct du pied, et contourné en spirale, en arrière; jamais de cuirasse, mais un collier formé tout autour du cou par le bord du manteau; tête surmontée d'une sorte de voile très large; tentacules au nombre de deux, les yeux diversement situés à leur base; cavité pulmonaire montrant son orifice sur le collier; organes de la génération séparés; anus près de l'orifice du poumon.

Coq. toujours complète, très enroulée, mince, et à bord externe, tranchant.

Tous fluviatiles.

\section{G. CXXX. Planonbe, planorbis, Brug.}

Anim. allongé, comprimé, grêle et très fortement enroulé; tête munie de deux tentacules contractiles, sétacés, fort longs et oculés à leur base interne; bouche munie supérieurement d'une dent en croissant, et inférieurement d'une masse linguale armée de petits crochets, surmontée d'une sorte de voile court et échancré; pied ovale et assez court; orifice de la respiration à gauche, sur le collier, et avoisiné par celui de l'anus; organes de la génération séparés de ce même côté, celui de l'organe mâle 
prês du tentacule, et celui des oeufs à la base du collier.

Coq. assez mince, sénestre, fortement enroulée dans le même plan; concave des deux côtés, la spire étant rentrante; ouverture arrondie, à bord tranchant et interrompu par la convexité du tour qui précède.

Le genre Planorbe offre une anomalie bien remarquable; c'est que son animal, de même que la coquille qui le renferme, est sénestre, et que par conséquent les orifices, au lieu d'être situés à droite, comme dans les autres Gastéropodes, sout à gauche. Les espèces de ce genre sont de tous les pays, et habitent sans exception dans les eaux douces. 11 y en a aussi plusieurs à l'état fossile.

Nous connaissons des individus du leucostoma recueillis à Seize, près Bordeaux, par M. Durieu, qui offrent cette singulière particularité que l'animal se renferme dans sa coquille au moyen d'un épiphragme analogue à celui des Hélices.

G. CXXXI. Limére, limncea, Lam.

Anim. de forme ovale, plus ou moins spiral; tête munie de deux tentacules aplatis, triangulaires, portant les yeux à leur base, du côté interne; bouche munie d'une pièce supérieure pour la mastication, surmontée d'une sorte de voile assez court; pied ovale, bilobé antérieurement, rétréci postérieurement; orifice de la cavité pulmonaire au côté droit, sur le collier, en forme de sillon, et pouvant être recouvert par un appendice charnu qui le borde inférieurement; anus tout à côté, organes de la génération distans, l'orifice de la verge étant sous le tentacule droit, et celui de la vulve à l'entrée de la cavité pulmonaire.

Coq. mince, fragile, ovale oblongue, à spire plus ou moins aiguë et allongée, à ouverture plus haute que large, ovale, quelquefois très grande, à bord 
tranchant, non continu par l'effet de la convexité du tour précédent; un pli oblique à la columelle.

Ce genre très voisin du précédent, mais qui s'en distingue si bien cependant, renferme un assez bon nombre d'espèces d'Europe; il en existe aussi dans l'Amérique septentrionale, et nous en avous rapporté une des îles du cap Verd et une autre beaucoup plus grande des Indes orientales. Les Limnées n'habitent que les eaux douces et tranquilles, où on les voit quelquefois, ainsi que les Planorbes et les Physes, venir nager à la surface dans une position renversée. On en connait aussi plusieurs à l'état fossile.

\section{G. GXXXII. Physe, physa, Drap.}

Anim. de forme ovale, plus ou moins spiral; tête munie de deux tentacules longs, sétacés et oculés à leur base interne; manteau offrant deux lobes digités sur ses bords, qui peuvent se recourber et recouvrir en grande partie la coquille; le pied long, arrondi antérieurement, aigu postérieurement; le reste de l'organisation extérieure comme dans les Limnées, à l'exception que les orifices sont généralement à gauche.

Coq. généralement sénestre, ovale, allongée, ou presque globuleuse, lisse, mince et très fragile; l'ouverture ovale, un peu rétrécie en arrière; bord droit tranchant (I), la columelle un peu torse, mais sans pli ; la spire plus ou moins aiguë et allongée, le dernier tour plus grand que les autres réunis.

Les Physes, si semblables aux Limnées dans leur organisation, habitent avec elles dans les eaux douces et tranquilles. Leur animal se distingue assez bien par la forme de ses tentacules, de même que la coquille par sa disposition généralement sénestre, comme celle des Planorbes. Il paraît cependant, d'après $M$. de Blainville, qu'il en existe

(I) Nous avons vu quelquefois un petit bourrelet interne au bord droit de certaines espèces de Physes et de Limnées. 
des espèces qui sont dextres. Il y a plusieurs Physes en Europe, dans l'Amérique septentrionale, en Afrique, puisqu'il est probable que le Bulin d'Adanson en est une, et nous en avons rapporté de Bourbon et de l'Ile-de-France.

\section{$6^{\text {C ORDRE. }}$}

\section{PULMONÉS OPERCULÉS, Fér.}

Trachélipodes colimacés, Lam.; Pectinibranches, Cuv.; Chismobranches cricostomes, Blainv.; Pneumopomes, Latreille.

Anim. muni d'un pied pour ramper, point de branchies, mais une cavité pulmonaire recevant le fluide ambiant par une grande solution de continuité, placée au-dessus de la tête; les tentacules au nombre de deux; les organes de la génération sur des individus différens.

Coq. extérieure, complète, spirale, globuleuse ou conique.

Operc. calcaire ou corné.

Tous terrestres.

Cet ordre a été établi par M. de Férussac aux dépens des Pectinibranches de M. Cuvier et pour le genre Cyclostome seulement; mais depuis le premier de ces savans a dû y réunir le genre Hélicine dont il a fait connaître l'auimal, et qui était autrefois confondu avec les Colimacés de M. de Lamarck. Aujourd'hui, les Pulmonés operculés établissent assez bien le passage des Pulmonés aux Pectinibranches, puisqu'ils tiennent des premiers sous le rapport des organes de la respiration, et des seconds sous celui de la séparation des sexes sur des individus différens.

$$
\text { I TO FAMLLLE. }
$$

LES HÉLICINES, Fér.

$$
\text { Hélicinides, Lat. }
$$

Anim. muni d'un collier, deux tentacules filiformes portant les yeux à leur base externe, sur des tubercules. 
Coq. plus ou moins globuleuse, à ouverture demiovale, à columelle transverse et amincie.

Operc. corné, quelquefois calcaire au-dehors.

M. de Férussac a établi les deux familles des Hélicines et des Turbicines pour deux genres assez voisins, et qu'il serait peut-être plus convenable de réunir en une seule, leur différence n'étant réeliement bien remarquable que dans leur enveloppe testacée.

\section{G. GXXXIII. Hélicres, helicina, Lam. olggyra, Say.}

Anim. très spiral, muni d'une tête proboscidiforme et d'un mufle bilabié; tentacules filiformes portant les yeux à leur base externe, sur des tubercules; pied court, arrondi, avec un sillon transversal antérieur; cavité pulmonaire s'ouvrant en avant du manteau par une grande fente transversale.

Coq. subglobuleuse ou conoïde, un peu déprimée, non ombiliquée, à spire basse, à ouverture demiovale ou presque ovale, le péristome réfléchi, en bourrelet, le bord gauche élargi sur l'ombilic, qu'il recouvre entièrement; la columelle calleuse transverse et planulée.

Operc. corné quelquefois légèrement, calcaire à l'extérieur, à accroissemens concentriques.

Le genre Hélicine a été établi par M. de Lamarck pour de petites coquilles qu'il rangeait parmi ses Colimacés; M. de Férussac, qui le premier en a connu l'animal, l'a porté à sa véritable place. Elles sont exotiques et assez nombreuses en espèces. Il y en a de fossiles. On doit rapporter à ce genre les Ampullines de M. de Blainville et r'Olygyra de M. Say.

$2^{\text {e FAMILLE. }}$

LES TURBICINES, Fér.

Anim. sans collier, pourvu de deux tentacules ooulés à leur base extérieure. 
Coq. conoïde, plus on moins élevée, à ouverture subarrondie et à bords continus.

Operc, calcaire.

\section{G. CXXXIV. Fénussine, ferussina, Grateloup. Strophostome, Deshayes.}

Anim. inconnu.

Coq. ovale, globuleuse; ouverture ronde, bordée , oblique, simple, sans dents, retournée du côté de la spire; un ombilic plus ou moins grand. Operc.?

M. Grateloup a établi ce genre pour une coquille fossile de Dax, qui semble au premier abord très voisine des Anostomes, mais que ce naturaliste croit, d'après l'examen de son ouverture, devoir plutòt rapprocher des Cyclostomes; nous partageons cette opinion, que la connaissance de l'opercule peut seule confirmer. Depuis lors, M. Deshayes n'ayant, sans doute, pas eu connaissance de la publication de ce genre dans la première liviaaison du Bulletin de la Société linnéenne de Bordeaux, l'a égalemeut publié, mais bien plus tard, sous le nom de Strophostome. Nous avons dû rétablir la première dénomination affectée à cette coquille. Les espèces de ce genre, au nombre de trois ou quatre, sont toutes fossiles.

\section{G. CXXXV. Gyclostome, cyclostoma, Lam.}

Anim. très spiral, muni d'une tête proboscidiforme portant deux tentacules cylindracés, renflés à leur sommet, contractiles et oculés à leur base externe; pied allongé et oblong; cavité pulmonaire communiquant à l'extérieur par une large fente à la partic supérieure et antérieure du manteau; position de l'organe mâle indiquée par un appendice tentaculiforme, situé au côté droit.

Coq. conoïde, discoïde ou turriculée, plus ou moins élevée, à sommet aigu ou mamelonné, ayant tous 
les tours arrondis; ouverture ronde, à bords continus et réfléchis.

Operc. calcaire, à accroissement concentrique, le sommet subcentral.

Le genre Cyclostome renferme un grand nombre d'espèces, la plupart exotiques; il y en a également, à l'état fossile.

$$
\eta^{e} \text { ORDRE. }
$$

\section{PECTINIBRANCHES, Cuv.}

Trachélipodes, Lam.; Clismobranches, Blainv.

Anim. muni d'un pied pour ramper; des branchies pour respirer, en forme de peigne, attachées au plafond d'une cavité particulière, qui s'ouvre en avant et en dessus, entre le bord du manteau et le corps, par une grande solution de continuité ; deux tentacules, deux yeux diversement situés, quelquefois sur des pédicules; les organes de la génération séparés sur des individùs différens; l'orifice femelle au côté droit, à l'entrée ou dans la cavité branchiale; l'organe mâle au côté droit du cou, se réfléchissant généralement dans cette cavité; anus du même côté et en avant.

Coq. complète èt spirale, très variable dans sa forme, presque toujours externe, rarement interne. Operc. complet, plus ou moins rudimentaire ou nul.

L'ordre des Pectinibranches renferme le plus grand nombre de genres, les plus connus et les plus répandus dans les collections. Le travail de M. de Férussac, dans lequel ce savant a apporté d'heureuses innovations pour leur distribution, va plus particulièrement nous servir de guide.

Les Pectinibranches sont la plupart des animaux marins, vivant parfois à d'assez grandes profondeurs. Un petit nombre habite les eaux douces.

$$
\text { † r re orvision. }
$$

Un appendice membraneux, quelqucfois diversement 
frangé ou découpé, de chaque côté du corps, pour faciliter l'entrée de l'eau dans la cavité branchiale; généralement point de trompe.

A. Presque toujours un opercule. Celui-ci modelé sur l'ouverture de la cociuille.

\section{I re FAMILLE.}

\section{LES TURBINÉS, Fér.}

Anim. muni de deux tentacules subulés, contractiles; les yeux à leur base.

Coq. variable dans sa forme, à ouverture arrondie ou ovale, à bords peu ou point désunis, sans canal ni échancrure.

Operc. corné ou calcaire.

Fluviatiles ou marins.

G. CXXXVI. Paludine, paludina, Fér.

Anim. muni d'une tête proboscidiforme; tentacules coniques, allongés, quelquefois distans, portant les yeux à leur base extérieure, ceux-ci sur de petites éminences plus ou moins saillantes; bouche munie d'une masse ou d'un ruban lingual hérissé; pied oblong ou ovale, plus ou moins allongé, et portant généralement un sillon marginal en a vant; organe mâle au côté droit antérieur, ayant son orifice à la base du tentacule ou dans le voisinage de cet organe; anus du même côté.

Coq. variant avec les sous-genres suivans.

Operc. corné, rarement calcaire.

Le genre Paludine, tel qu'il a été proposé dans ces derniers temps par M. de Férussac, et tel que nous l'adoptons provisoirement aujourd'hui, est bien plus étendu que M. de Lamarck ne l'avait fait, puisque l'on y trouve maintenant réunis les Mélanies de cet auteur et deux genres nouveaux formés aux dépens des Sabots, les Rissoaires et les Littoriens; elles sont donc ou marines ou fluviatiles. Tous ces genres, devenus sous-genres, se distinguent comme il suit. 
I $^{\text {er }}$ S.-G. Paludine, paludina, Lam.

Anim. pourvu d'une bouche sans dents, mais contenant une petite masse linguale hérissée; tentacules contractiles; pied ovale, muni d'un sillon marginal à la partie antérieure; organe mâle très gros, renflant le tentacule droit, d'où il sort par un orifice situé près de sa base; anus à l'extrémité d'un petit tube au plancher de la cavité respiratrice.

Coq. épidermée, conoïde, à tours de spire arrondis, à sommet mamelonné; ouverture arrondie, ovale, anguleuse au sommet, à bords réunis, tranchans.

Operc. corné, à élémens concentriques, à sommet excentrique.

Ce sous-genre renferme un assez grand nombre d'espèces de tous les pays. On en connaît aussi quelques unes à l'état fossile. Les Paludines habitent les eaux douces et quelquefois saumâtres.

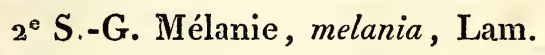

Anim. ayant un mufle proboscidiforme, demi-cylindrique, un peu échancré antérieurement; tentacules fliformes; pied ovale et très grand; manteau festonné en avant et à gauche.

Coq. épidermée, ovale-oblongue, à spire pointue, souvent allongée ou turriculée; ou verture ovale, évasée en avant, et à bord externe tranchant.

Operc. mince, subspiral, à élémens subradiés en dehors, rebordé en dedans.

Les Mélanies sont toutes fluviatiles et exotiques; elles paraissent surtout appartenir à l'Asie. On en a reconnu en Europe, mais à l'état fossile, et M. de Blainville parait disposé à croire qu'elles ne se rapportent point à ce sousgenre. M. Deshayes divise ainsi les Mélanies : 


$$
\text { Ier groupe. }
$$

Coq. ovale ou subturriculée.

M. amarula, etc.

$$
2^{\mathrm{e}} \text { groupe. }
$$

Coq. allongée, turriculée.

M. truncata, etc.

$$
3^{\mathrm{e}} \text { groupe. }
$$

Coq. dont l'angle inférieur est détaché.

M. costellata, etc.

$$
4^{\mathrm{e}} \text { groupe. }
$$

Coq. à ouverture bordée.

M. marginata, etc.

Les Mélanies ne conservent intact le sommet de leur spire que pendant leur jeune âge, bientôt il s'excorie et tombe.

3e S.-G. Rissoaire, rissou, Fréminville.

Anim. portant les tentacules coniques, latéraux et distans; pied court et rond.

Coq. oblongue ou turriculée, non ombiliquée, généralement remarquable par ses côtes longitudinales; ouverture entière, ovale, oblique, évasée, sans canal, ni dents, ni plis; les deux bords réursis ou presque réunis, le droit renflé et non réfléchi.

Operc. calcaire ou corné, rentrant assez profondément.

iM. de Fréminville a formé le genre Rissoaire pour une petite coquille marine de la Méditerranée qui, avec un animal assez semblable à celui des sous-genres précédens, se distingue par l'ouverture de la coquille et la prèsence presque constante de côtes longitudinales. Nous ne pensons pas que l'on puisse admettre les Rissoaires comme genre, mais bien comme sous-genre; elles prennent alors leur place à côté des Mélanies et dans le genre Paludine de M. de Férussac. Il y en a de fossiles. M. de Blainville divise ainsi co sous-genre: 


\section{I er groupe.}

Coq. turriculée et cotelée.

R. acuta, etc:

$$
2^{e} \text { groupe. }
$$

Coq. subturriculée et cotelée.

$R$. cortata, etc.

$$
\text { 3e groupe. }
$$

Coq. subturriculée, parfaitement lisse.

R. hyalina, etc.

$$
4^{e} \text { groupe. }
$$

Coq. subglobuleuse.

R. cancellata, etc.

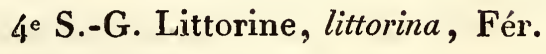

Anim. muni de tentacules grêles et allongés; bouche pourvue seulement d'un ruban lingual; pied oblong, marqué d'un sillon marginal à la partie antérieure; organes de la génération se termiminant, dans les deux sexes, au côté droit, à l'entrée de la cavité branchiale, tout près de l'anus.

Coq. toujours épaisse, globuleuse, conique ou subturriculée, non ombiliquée, à ouverture parfaitement ronde dans la direction de l'axe, et un peu évasée en avant.

Operc. corné.

M. de Férussac a établi le genre Littorine pour des mollusques tous marins, suffisamment distincts, comme sousgenre de ceuxx qui l'avoisinent. Le type en est la $L$. littoralis, M. Alcide Dorbigny a préparé une monographie de ce sous-genre, dans laquelle il fait connaître un très grand nombre de petites espèces vivantes et fossiles.

G. CXXXVII. Turritelle, turritella, Lam. Anim. muni d'une trompe, et supérieurement d'une frange en forme de voile; tentacules longs, très 
fins à leur extrémité, renflés à leur base, avec les yeux en dehors et sur un renflement; pied découpé à sa circonférence? et bordé en avant par un bourrelet ridé transversalement.

Coq. turriculée, pointue, un peu mince, généralement striée dans le sens de la longueur des tours de spire, qui sont nombreux; ouverture arrondie, entière, avec les bords désunis supérieurement, celui de droite mince.

Operc. corné, à élémens concentriques.

Les Turritelles sont presque toutes exotiques; mais nous en avons en France un bon nombre à l'état fossile.

\section{G. CXXXVIII. Рвото, proto, Def. .}

Anim. inconnu.

Coq. turriculée, allongée, à tours de spire nombreux, aplatis, avec une bande décurrente à la suture : ouverture oblique, arrondie, évasée, à bords désunis, le droit tranchant, le gauche très évasé.

Operc. inconnu.

M. Defrance a établi ce genre pour des coquilles fossiles.

G. CXXXVIII bis. Vermet, vermetus, Acauns. Vermiculaire, Lam.

Anim. vermiforme, conique, à tête peu distincte, muni d'une trompe garnie à son extrémité de plusieurs rangs de crochets; deux tentacules un peu aplatis et coniques, porteunt les yeux à leur base extérieure; manteau formant un bourrelet en forme d'anneau, à l'endroit où sort la partie postérieure du corps; pied cylindrique, avec deux longs filets tentaculaires à la racine antérieure; orifice de l'organe respiratoire en forme de trou, percé au côté droit du bourrelet du manteau.

Coq. irrégulièrement spirale, conique, mince, ayant les tours de spire plus ou moins complétement désunis et écartés; ouverture droite, circulaire, 


\section{MB́THODIQUE, etc.}

le péristome continu et tranchant; quelques cioisons vers le sommet; libre ou adhérente par entrelacement.

Operc. corné.

Ce genre singulier, dont on doit la counaissance à Adanson, ne renferme qu'une espèce bien caractérisée. L'animal, qui est véritablement un Gastéropode, ne saurait ramper, puísque la coquille est fixée; aussi son pied, quoique distinct, ne paraît pas disposé pour cet usage. L'espèce d'Adanson est le $V$. lombricalis. Nous en possédons de fossiles.

\section{G. CXXXIX. Silrquarre, siliquaria, Lam.} Tenagode, Guettard; Anguinaire, Schumacher.

Anim. très allongé, tourné en spirale, muni d'un tortillon assez court et d'un pied musculaire très charnu, qui porte supérieurement une sorte d'appendice (peut-être une ventouse?) très comprimé; tête distincte, portant deux petits tentacules cylindriques et obtus à leur extrémité, avec les yeux à leur base extérieure; manteau fendu supérieurement dans toute sa longueur jusqu'au commencement du tortillon, de manière à présenter deux lobes latéraux, l'un qui est à droite, en forme de frange étroite, bordée en dedans par un sillon, et s'étend de la tête au tortillon; l'autre, qui est a gauche, beaucoup plus large dans toute son étendue, mais surtout en avant, commence après la tête, et se rétrécit insensiblement jusqu'en arrière; branchies en forme de filamens simples, assez rigides, fixés à la face interne et sur toute la longueur du lobe gauche; terminaison des organes de la génération indiquée par une petite échancrure sur ce même lobe? anus en arrière.

Coq. tubuleuse, contournée en spirale lâche et irrégulière, souvent assez régulière vers le sommet, cloisonnée; l'ouverture circulaire, à bords tranchans, désunis par une scissure ou une série longi- 
tudinale de petits orifices se prolongeant jusqu'auprès du sommet.

Operc. en forme de cône droit, tronquué, composé de lamelles cornées, empilées les unes sur les autres.

Le genre Siliquaire avait été rangé parmi les Annélides arénicoles par M. de Lamarck. M. de Blainville, le premier, a pensé que ce pouvait être la coquille d'un mollusque; mais c'est $M$. Audouin qui a donné à cette dernière opinion toute la solidité possible par l'observation qu'il a faite récemment de l'animal d'une Siliquaire rapportée des mers de l'Inde par M. le docteur Busseuil, chirurgien-major de la frégate la Thétys. M. Audouin va faire connaître plus en détail, dans les Annales des Sciences naturelles, le résultat de ses recherches sur cet intéressant mollusque, qu'il retirera sans doute de l'ordre des Pectinibranches, dans lequel nous pensons qu'il ne peut plus rester, non plus, peut-être, que le genre suivant. Il existe plusieurs espèces de Siliquaires vivantes et fossiles, et l'on vient d'en découvrir une dans les mers de Sicile.

\section{G. CXL. MaGile, magilus, Montf.}

Anim. inconnu.

Coq. Jeune; fragile, épidermée, piriforme, ventrue, à spire courte de trois à quatre tours; ouverture plus longue que large, oblongue, sans échancrure en avant, où les bords forment cependant un angle. Adulte; le dernier tour abandonnant tout à coup la révolution spirale et régulière pour former un tube allongé, irrégulièrement sinueux, conique, comprimé latéralement, surtout du côté de la base de la coquille, où il est comme caréné, et arrondi du côté du sommet; son ouverture devient alors ovale, anguleuse et à bords continus; la surface extérieure est sillonnée dans le sens de la longueur des tours, et toute lamelleuse, dans le sens contraire, par le rapprochement des anneaux d'accroissement. Operc.?

Les Magiles ont été classés par M. de Lamarck parmi es 
Annélides, farillte c'es Serpulées. M. de Blainville est le premier qui les ait admis parmi les Mollusques, et nous arons été frappé comme ce naturaliste, lorsque nous cherchions dans l'Inde leur animal, de l'analogie qu'ils présentent, non seulement avec les Vermets, mais encore avec plusieurs autres genres de Pectinibranclies. Cette analogie est surtout facile à saisir, quand on a sous les yeux un individu jeune dont la partie tubuleuse n'est pas encore commencée. Nous avons eu occasion de voir quelques fragmens de l'animal, et nous croyons pouvoir assurer qu'il est bien gastéropode.

Le Magile étant très jeune s'établit dans les excavations de certains madrépores; mais ceux-ci venant bientôt à grossir leur masse autour de lui, il est obligé, pour se ménager une ouverture au-dehors, de construire un tube dont l'orifice se maintient toujours par de nouveaux accroissemens, au niveau de la surface du polypier qui le recèle. Ainsi, l'épaisseur qu'acquiert celui-ci durant la vie du mollusque, détermine toujours la longueur de ce tube.

L'animal abandonne promptement la partie spirale pour se porter en avant dans la partie tubuleuse à mesure qu'il la forme; il ne laisse point de cloisons derrière lui; mais il dépose lentement une matière calcaire qui, pénétrant jusqu'au sommet de la coquille, se durcit, et en devient le moule parfait. Nous avons rencontré plusieurs de ces moules qui avaient une grandeur considérable, et se trouvaient encore euchâssés dans les Madrépores, ne montrant à leur surface que des fragmens adhérens du test qui les enveloppait. Ces moules très compactes et très durs sont de la même nature que les Polypiers dans lesquels ils ont été formés, leur cassure est saccharoïde et radiante. La coquille, sans ce moule, ne se trouve jamais que dans le jeune âge, parce que, dès que l'animal commence son tube, il commence aussi à remplir la partie spirale.

\section{G. CXLI. Valvée, valuata, Muller.}

Anim. muni d'une tête très distincte, prolongée en une sorte de trompe; tentacules fort longs, cylindracés, obtus, très rapprochés; yeux sessiles 
au côté postérieur de leur base; pied bilobé en avant; branchies longues, pectiniformes, plus ou moins exsertiles hors de la cavité, celle-ci largement ouverte et pourvue, a droite de son bord inférieur, d'un long appendice simulant un troisième tentacule; organe mâle se retirant dans la cavité respiratrice.

Coq. discoïde ou conoïde, ombiliquée, à tours de spire cylindracés, à sommet mamelonné; ouverture ronde ou presque ronde, à bords réunis, tranchans.

Operc. corné, rond, à élémens concentriques et circulaires.

Ce genre ne renferme que des mollusques d'eau douce, tous d'Europe.

G. CXLII. Natice, natica, Adans. E.t Polinice, Montf., Rotelle, Lam.

Anim. muni d'une tête échancrée antérieurement, avec deux tentacules très longs et pointus, un peu aplatis à leur base, où se trouvent, du côté extérieur, des yeux sessiles; bouche munie d'une dent labiale, sans langue; pied court, profondément et transversalement bilobé en avant, montrant en arrière un lobe appendiculaire qui porte l'opercule. Coq. lisse, sans épiderme, subglobuleuse ou orbiculaire, à spire basse, à ouverture demi-ronde, le bord gauche oblique, non denté, muni d'une callosité qui modifie plus ou moins l'ombilic, et le recouvre quelquefois en entier, le bord droit tranchant et lisse à l'intérieur.

Operc. calcaire ou corné, semi-spiré, à sommet latéral sans apophyse à sa base.

Les Natices sont toutes des coquilles marines, assez nombrenses en espèces et recherchées dans les collections, de 
même que la plupart de celles qui ront suivre. On peut former plusieurs groupes très distincts dans ce genre.

$$
\text { ler }^{\text {er groupe. }}
$$

Coq. globuliforme; opercule calcaire. N. castanea, etc. (Natices, Montf,)

$$
2^{e} \text { groupe. }
$$

Coq. ovale, mamelonnée; opercule corné. N. mamilla, etc. (Polinice, Montf.)

$$
3^{c} \text { groupe. }
$$

Coq. surbaissée, opercule corné.

N. lineolata, etc. (Rotelles, Lam.)

Les deux premiers groupes peuvent encore se subdiviser par la considération de l'ombilic, qui est toujours plus ou moins complétement fermé par la callosité de la columelle.

\section{$2^{\text {e FAMILLE. }}$}

\section{LES TROCHOÏDES, Cuv.}

Anim. muni de deux tentacules contractiles; les yeux pédonculés à leur base externe.

Coq. très variable dans sa forme, à ouverture quelquefois à bords désunis, mais sans former de canal, et n'ayant que très rarement un sinus à sa partie antérieure.

Marins et d'eau douce.

a) Ouverture de la coquille sans sinus en avant.

G. CXLIII. Navigella, navicella, Lam. Septaire, Fér.; Cimber, Montf.

Anim. ovale, non spiral, muni d'une tête avancée, semi-lunaire et déprimée, portant deux tentacules contractiles, coniques, allongés, avec les yeux à leur base extérieure, sur de petites éminences; 
bouche fendue en long, sans dent supérieure, mais ayant une langue à crochet, prolongée jusque dans la cavité viscérale, et fendue à son origine antérieure; pied grand et elliptique, à bords minces, très avancé antérieurement, attaché de chaque côté dans toute sa partie postérieure, au reste du corps, de manière à former une sorte de poche ouverte transversalement en arrière; une seule grande branchie oblique; orifice de l'oviducte dans la cavité branchiale, celui du canal déférant à la racine et en dessous de l'organe excitateur, situé en avant du tentacule droit; anus à l'extrémité d'un tube flottant à droite, au plafond de la cavité branchiale.

Coq. épidermée, elliptique ou oblongue, patelloïde, à sommet non spiré, médian, abaissé sur le bord postérieur; concave en dessous, sans columelle, mais à bord gauche aplati, tranchant, sans dent, et simulant un commencement de cloison; bord droit ou antérieur, mince et tranchant; impression musculaire en forme de fer à cheval ouvert en avant et interrompu en arrière.

Operc. calcaire, mince, quadrilataire, fixé comme à l'ordinaire à la partie supérieure du pied, mais caché dans la cavité qui se trouve entre celui-ci et la masse des viscères; montrant un sommet distinct, d'où partent les rayons divergens; une épine latérale et postérieure.

Le genre Navicelle, si remarquable par la singulière disposition de son opercule, ne renferme encore qu'un petit nombre d'espèces, toutes des Grandes-Indes nu de leurs Archipel; elles habitent uniquement les eaux donces, les plus claires, et se fixent sur les rochers à la manière des Patelles. Elles rampent très bien, et n'adoptent point une place comme font celles-ci. Nous avons caractérisé l'animal d'après des individus vivans de la $N$. elliptica. Cette espèce, très commune à l'íle de Bourbon, s'emploie pour faire du bouillon aux malades. 
G. CXLIV. Nérrte, nerita, Lin. Et Péloronte, Ocken, Clithon, Montf., Piléole, Sow. Anim. spiral, muni d'une tête un peu avancée en mufle; bouche sans mâchoire, mais avec une langue denticulée, prolongée dans la cavité viscérale; tentacules coniques, fins et assez allongés, portant les yeux à leur base extérieure, sur une protubérance; pied rond ou ovale; une seule grande branchie; organe excitateur mâle au côté droit, en avant du tentacule; anus dans la cavité branchiale.

Coq. épaisse, souvent épidermée, semi-globuleuse, à spire peu ou point saillante, aplatie en dessous, et sans ombilic; ouverture semi-lunaire, à bord droit, uni, denté ou crénelé à l'intérieur, le gauche septiforme, tranchant horizontal denté ou non denté; impression musculaire en forme de fer à cheval incomplet.

Operc. calcaire, subspiral, à sommet marginal, à l'extrémité gauche; une ou deux apophyses d'adhérence musculaire à son bord postérieur.

Le genre Nérite, tel qu'il est actuellement, comprend les Nérites et les Néritines de Lamarck, c'est-à-dire le genre Nérite, tel que l'avait établi Linné. M. de Lamarck avait sans doute séparé à tort les Nérites d'eau douce des Nérites marines en leur affectant à chacune des caractères pris sur la coquille, tels que celui des dentelures à l'intérieur du bord droit; car sa Neritina viridis, qui n'a point ce caractère, est une espèce marine, et non des rivières comme il le prétend; nous l'avons fréquemment trouvée sous les rochers baignés par la mer à la Martinique, ainsi "qu'à Madagascar où elle offre une variété bien plus grosse.

Le genre Nérite est très nombreux, et renferme des coquilles extrêmement variées dans leurs couleurs comme dans leur forme. M. Lesson en a rapporté une espèce des terres australes bien distincte de toutes celles que uous connaissious jusqu'à présent, et qui s'est offerte en assez grande abondance sur les arbres. La connaissance de ce fait doit 
engager plus que jamais à ne point séparer les espèces marines des espèces fluviatiles. M. de Blainville, qui a adopté, comme M. de Férussac, la réunion de ces genres, propose de diviser ainsi les Nérites d'après les caractères de leur coquille.

$$
\dagger \mathrm{I}^{\mathrm{re}} \text { section. }
$$

Le bord droit denté. (Nérite, Lam.)

$$
{ }_{1}^{\mathrm{er}} \text { groupe. }
$$

Une seule dent médiane au bord gauche. (G. Péloronte, Ocken.)

N. peloronta, etc.

Deux dents.

$$
2^{\mathrm{c}} \text { groupe. }
$$

N. exuvia, etc.

$$
3^{\text {e }} \text { groupe. }
$$

Trois ou quatre dents.

N. lineata, etc.

$$
\text { †† } 2^{\mathrm{e}} \text { section. }
$$

Le bord droit non denté. (G. Néritine, Lam.)

$$
4^{\mathrm{e}} \text { groupe. }
$$

Coquille moins épaisse, à bord droit tranchant, l'opercule très oblique. (G. Néritine, Lam.)

N. fluviatilis, etc.

$$
\text { 5e groupe. }
$$

Le bord columellaire denté, coquille pourvue d'épine. (G. Clithon, Montf.)

$N$. corona, etc.

$$
6^{\mathrm{e}} \text { groupe. }
$$

Le bord columellaire denté; les deux extrémités dus bord droit se prolongeant beaucoup au-delà de l'ouverture, et formant avec la callosité qui recourre le bord columellaire des sortes d'auricules produites par le lobe tentaculaire de l'animal.

N. auriculata, etc.

$$
7^{\mathrm{e}} \text { groupe. }
$$

Coquille calyptroïde à sommet supérieur vertical 
spiré; le dernier tour formant toute la base de la coquille, et occupé en dessous par une large callosité qui recouvre quelquefois toute la spire. (G. Velate, M.)

N. perversa, etc.

$$
8 \text { e graitpe. }
$$

Coquille patelloïde, allongée, non symétrique, à sommet dorsal et non spiré. (G. Piléole, Sowesby.) $N$. alta villensis, etc.

Il y a plusieurs espèces de Nérite à l'état fossile; les deux dernières sont du nombre.

\section{G. GXl.V. Ampuldaine, ampullaria, Iam.}

Anim. spiral, globuleux, muni d'une tête large, portant quatre tentacules, deux grands supérieurs, coniques, avec un pédoncule pour les yeux, à leur base extérieure; bouche verticale entre deux lèvres disposées en fer à cheval, et formant une espèce de mufle; point de mâchoire, mais un ruban lingual hérissé, non prolongé dans la cavité abdominale; pied ovale avec un sillon transverse en avant; cavité respiratrice très vaste et partagée en deux par une cloison horizontale incomplète. Coq. épidermée, généralement assez mince, globuleuse, ventrue, ombiliquée; spire très courte, le dernier tour beaucoup plus grand que tous les autres ensemble; ouverture ovale, plus longue que large, à bords réunis, le droit tranchant.

Operc. corné, rarement calcaire, mince, non spiré, à élémens concentriques, à sommet submarginal inférieur.

Les Ampullaires sont des coquilles toutes exotiques dont les animaux vivent dans les fossés d'eau douce, et quelquefois viennent sur leurs bords respirer l'air libre. Leurs œufs sont comme des petites vésicules arrondies, souvent agréablement colorés de vert et réunis par groupes sur les 
tiges des plantes aquatiques. Ce genre renferme plusieurs espèces vivantes, toutes des pays chands; il y en a aussi beaucoup de fossiles.

\section{G. CXLVI. Janthine, janthina, Lam.}

Anim. pourvu d'une tête très grosse et d'un mufle proboscidiforme, à l'extrémité duquel est la bouche; celle-ci munie de deux lèvres verticales, subcartilagineuses, armées d'aiguillons recourbés en dedans, longs et très aigus, et d'un renflement lingual; deux tentacules coniques, pointus, peu contractiles et très distans, portant chacun à leur base extérieur un pédoncule assez long, oculé au-dessous de son extrémité ; pied ovale, divisé en deux parties, l'antérieure concave et en forme de ventouse, la postérieure aplatie, épaisse et charnue; des appendices natatoires, latéraux, assez grands et frangés; cavité respiratrice très ouverte, et contenant deux peignes branchiaux; orifice de l'ovaire dans le fond de cette cavité; organe excitateur mâle très petit, au côté droit.

Coq. ventrue, globuleuse ou conoïde, très mince, à spire basse, le dernier tour plus grand que tous les autres réunis; ouverture grande, subtriangulaire, à bords désunis, la columelle droite, longue, formant tout le bord gauche; bord droit tranchant, souvent échancré dans son milieu. Toutes les espèces connues jusqu'à ce jour sont de couleur violette.

Operc. modifié en un appendice vésiculeux qui sert à suspendre l'animal à la surface de l'eau, et qui adhère à la partie postérieure et charnue du pied.

Le genre Janthine, connu depuis très long-temps, ne renferme que des animaux pélagiens; mais que l'on rencontre fréquemment sur nos côtes où la tempête et les courans les jettent en grand uombre; ils ne rampent point, n'étant pas organisés pour habiter sur les fouds. M. Eve- 
rard Home dit que ce mollusque renferme ses œufs dans une bande glaireuse dont il entoure sa coquille; nous ne pouvons pas croire qu'il ait méconnu l'appendice vésiculeux ; mais ce qu'il y a de certain, c'est que la Janthine dépose ses œufs quelquefois en nombre considérable, comme nous avons eu occasion de le remarquer, sous cet appendice vésiculeux même, qu'elle les attache par le moyen de petits pédicules, et qu'elle les abandonne arec cet organe aérien chargé alors de leur conservation. Il est possible qu'à cette époque les appendices natatoires de son manteau, étant suffisamment développés, lui permettent de s'en servir pour la natation, et suppléer ainsi à la perte qu'elle vient de faire, ou bien doit-on supposer que ces animaux ont la faculté de remplacer cet organe après l'avoir abandonné. Les Janthines répandent toutes une liqueur de couleur laqueuse, dont l'intensité conserve quelque durée. On a fait dans ce genre un grand abus des distinctions spécifiques. M. de Blainville penche à croire que celles qui sont échancrées appartiennent à des femelles.

\section{G. CXLVII. Lrtrope, litiopa, Rang.}

Anim. transparent, spiral, muni d'un pied assez court et étroit, et d'une tête portant deux tentacules coniques, allongés, avec les yeux à leur base extérieure.

Coq. peu épaisse, cornée, légèrement épidermée, un peu transparente, conoïde, à tours de spire un peu arrondis, le dernier plus grand que tous les autres rêunis, à sommet pointu, sillonné longitudinalement; ouverture ovale plus large en avant qu'en arrière, à bords désunis, le droit simple se réunissant au gauche, sans former d'échancrure bien distincte, mais seulement un contour profond qui en tient lieu, le bord ganche rentrant en dedans pour former une saillie avec l'extrémité antérieure de la columelle, qui est unie, arrondie, arquée et un peu tronquée en avant.

Operc. Point d'opercule. 
Nous avons établi ce genre dans les Annales des Sciences maturelles pour un mollusque pélagien fort curieux par ses mœurs. Depuis plusieurs années, nous en avions observé la coquille; mais le temps ne nous avait pas permis d'en étudier l'animal; c'est M. le capitaine de frégate Bellanger qui l'a connu le premier; malheureusement il n'en a point étudié l'organisation extérieure, ainsi tout restait à faire à ce sujet; cependant il avait observé ce fait singulier, que l'animal qui vit sur les plantes errantes, s'en écarte quelquefois en s'y tenant fixé par un fil. Cet officier a eu la complaisance de nous donner avec ces renseignemens plusieurs individus conservés dans l'esprit de vin, et nous avons pu non seulement y reconnaître quelques uns des caractères extérieurs, mais encore nous assurer de l'exactitude de ses observations par la rencontre à la pointe de notre scalpel de petites masses glaireuses qui nous ont paru tenir au pied, et qu'il a été facile de faire filer à une assez grande longueur. Nous avons vainement cherché l'opercule; il nous a été impossible de le trouver, ce qui établit une grande différence entre ce mollusque et les Phasianelles. Nous en avons distingué deux espèces assez faciles à caractériser, par la coquille, car les animaux nous ont paru semblables; elles sont toutes de l'Océan. Le genre Litiope vient prouver, comme quelques autres, qu'il n'est pas possible d'établir des divisions fondées sur la présence ou l'aksence de l'opercule.

\section{G. CXLVIII. Phasianele, phasianella, Lam.}

Anim. oblong, spiral; tête pourvue en avant de deux doubles lèvres frangées, formant un voile audessus de la bouche; celle-ci verticale, munie de deux petites plaques cornées et d'un ruban lingual, hérissé et prolongé en spiral dans la cavité abdominale; tentacules longs et coniques, avec deux pédoncules de méme forme, situés à leur base externe, et portant les yeux; pied oblong; des membranes latérales frangées, et présentant de chaque côté trois appendices tentaculiformes; cavité branchiale partagée en deux par une cloison dont chaque paroi porte une série de feuillets. 
branchiaux; anus ouvert à l'extrémité d'un petit tube sous le bord antérieur et au côté droit de cette cloison.

Coq. assez épaisse, lisse, ovale ou conique, à spire pointue; ouverture ovale, plus longue que large, à bords désunis supérieurement, le droit tranchant; columelle lisse, comprimée, se fondant un peu avec le bord gauche, et offrant intérieurement une callosité longitudinale.

Operc. calcaire, subspiré, à sommet terminal.

Les Phasianelles, si recherchées avant le voyage de Péron, forment encore l'ornement des cabinets; ce sont des coquilles marines presque toutes des mers de la NouvelleHollande et des grandes Indes; il paraît cependant qu'il y en a sur les côtes de l'Amérique méridionale, et nous en connaissons une de la Méditerranée. C'est à M. Cuvier que l'on doit la connaissance de l'animal, que depuis nous avons eu également occasion de remarquer. On en connait aussi de fossiles.

\section{G. CXLIX. ToupIe, trochus, Lin.]}

Anim. muni d'une tête distincte, avec une bouche sans mâchoire supérieure, mais pourvue d'une langue en forme de ruban et contournée en spirale dans la cavité viscérale; deux tentacules plus ou moins allongés; yeux à leur base extérieure, sur de petits renflemens subpédonculés; pied généralement court et arrondi; les appendices membraneux latéraux, digités, ou diversement frangés; un ou deux peignes branchiaux de forme inégale; organe femelle à droite dans la cavité branchiale, celui du mâle du même côté, se terminant par une sorte de languette triangulaire, soutenu par un petit osselet; anus à la droite de la cavité respiratrice.

Coq. épaisse, très souvent nacrée à l'intérieur, trochoïde, à spire parfois surbaissée et parfois al- 
longée, pointue au sommet, souvent tranchante ou carénée, ombiliquée ou non; ouverture déprimée, anguleuse ou arrondie, à bords souvent désunis, le droit presque simple et toujours tranchant, la columelle arquée, souvent torse.

Operc. corné ou calcaire, spiral.

M. de Férussac ramenant au genre Trnchus des coquilles qu'on en avait séparées par des distinctions génériques, et dont les animaux ne montrent aucune différence notable, nous adopterons la réunion proposée par ce savant. Toutes les Toupies sont des coquilles marines; elles fournissent un nombre considérable d'espèces, soit vivantes, soit fossiles, que l'on recherche beaucoup dans les collections; elles sont de toutes les mers.

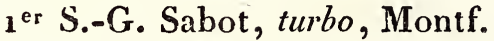 Et Lacuna, Turton.}

Annim. muni d'une tête un peu proboscidiforme, avec des tentacules grêles; un sillon transversal en avant du pied; les membranes latérales offrant quelquefois de petits appendices tentaculaires différens de nombre et de forme.

Coq. nacrée à l'intérieur, globuleuse, conoïde ou subturriculée, point ombiliquée ni carénée; ouverture ronde; columelle arquée, non tordue et sans troncature à sa base.

Operc. calcaire, à spire visible du côté interne seulement, l'externe épaissi et souvent guilloché.

Dans ce sous-genre, Mi. de Férussac ne comprend que les Sabots de Montfort, c'est-à-dire les espèces de Lamarck. qui n'ont point d'ombilic.

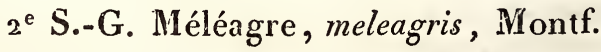

Anim. semblable au précédent.

Coq. semblable à celle des Sabots, mais ombiliquée.

Operc. semblable. 
3e S.-G. Monodonte, monodonta, Lam.

Anim. semblable aux précédens, mais généralement plus orné, et ayant sonvent de chaque côté trois filets aussi longs que ses tentacules.

Coq. subglobuleuse, ovale ou conoïde, à ouverture entière et arrondie, ombiliquée ou non; columelle arquée, tronquée à sa base, souvent munie d'une dent; bord droit quelquefois sillonné en dedans.

Operc. corné, à spire visible au-dehors.

Il y en a beaucoup à l'état fossile.

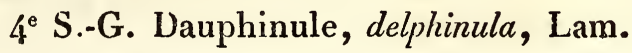

Anim. semblable aux précédens?

Coq. très épaisse, subdiscoïde ou conique, largement ombiliquée, à tours de spire arrondis, rudes ou anguleux, et quelquefois séparés; ouverture entière, ronde ou trigone, sans columelle, à bords complétement réunis, le plus souvent frangés ou munis d'un bourrelet.

Operc. calcaire, paucispiré, tuberculeux à l'extérieur.

Ce sous-genre ne renferme que peu d'espèces vivantes, mais aussi un assez grand nombre fossiles.

$$
\text { 5e S.-G. Éperon, calcar, Montf. }
$$

Anim. semblable aux précédens?

Coq. ombiliquée, très déprimée, à spire surbaissée; carénée, tranchante et radiée par la conservation d'un canal anguleux du bord droit; ouverture anguleuse.

Operc. corné, mince, à tours de spire nombreux.

Ce sous-genre ne renferme qu'un petit nombre d'espèces, parmi lesquelles plusieurs sont assez recherchées. Il y en a de fossiles. 


\section{$6^{\circ}$ S.-G. Fripière, phorus, Montf.}

Anim. semblable aux précédens?

Coq. ombiliquée, déprimée, à spire peu élevée; la base fort élargie et comme excavée par la grande saillie de l'angle du bord droit qui s'avance bien au-delà du bord columellaire arrondi ; agglutinante.

Operc. corné, spiral.

Ce sous-genre ne renferme qu'une seule espèce vivante. Il y en a une fossile qui lui est analogue. L'animal se remarque par la singulière propriété qu'il possède de s'entourer de cailloux et de débris de coquilles qu'il fixe à la paroi externe de son test.

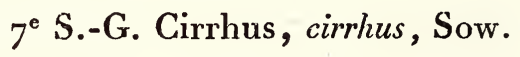

Anim. inconnu.

Coq. conique, non ombiliquée, un peu déprimée, à spire assez élevée, sans columelle, formant en dessous un entonnoir dont les tours sont joints. Operc. inconnu.

Ce sous-genre ne renferme encore que des coquilles fossiles.

$8^{e}$ S.-G. Cadran, solarium, Lam.

Anim. comme les précédens?

Coq. orbiculaire, en cône déprimé; ombilic très ouvert, crénelé sur le bord interne des tours de spire; ouverture presque quadrangulaire; point de columelle.

Operc. corné.

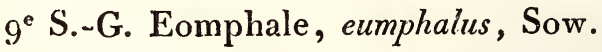

Anim. inconnu.

Coq. semblable à la précédente, mais plus conique, et sans crénelures au côté interne de l'ombilic.

Operc. inconnu. 
MÉTHODIQUE, etc.

Ce genre, établi par Sowesby, ne renferme que des espèces fossiles.

$10^{\circ}$ S.-G. Entonnoir, infundibulum, Montf.

Anim. très orné de franges à ses appendices membraneux.

Coq. conique, calyptriforme, très concave en dessous, les tours de spire aplatis; cavité formée par la lame septiforme de sa face inférieure.

Operc. corné, rond, spiral, à tours de spire rapprochés.

Ce sous-genre, très distinct des autres, ne renferme qu'un petit nombre d'espèces. Il y en a une belle à l'île de Bourbon que l'on vend au bazar pour la nourriture du peuple.

I I S.-G. Toupie, trochus, Lin.

Anim. comme dans la caractéristique du genre.

Coq. épaisse, nacrée à l'intérieur, conique, à spire pointue, quelquefois tranchante à sa base, non ombiliquée, à face inférieure plane ou presque plane; ouverture anguleuse ou subquadrangulaire, à bords désunis, le droit tranchant; columelle arquée et torse.

Operc. corné, mince, spiral, à tours de spire rapprochés.

Ce sous-genre renferme beaucoup d'espèces vivantes ou fossiles; les premières sont de toutes les mers.

$12^{*}$ S.-G. Télescope, telescopium, Montf.

Anim. inconnu.

Coq. sans ombilic, non nacrée, en cône très élevé; tours de spire nombreux, à stries décurrentes; extrémité de la columelle fortement tordue et dépassant l'origine du bord.

Operc. inconnu. 
Ce sous-genre ne renferme que l'espèce qui lui sert de type, et qui est de la mer des Indes.

\section{G. CL. Pleurotomaire, pleurotomaria, Def.} Et Sissurelle, Dorb.

Anim. inconnu.

Coq. conoïde ou subdiscoïde, quelquefois carénée, à spire peu convexe, munie d'un grand ombilic; ouverture plus ou moins ronde, avec une entaille profonde vers le milieu du bord droit.

Operc. inconnu.

Ce genre, établi par M. Defrance, ne renferme que des. coquilles fossiles; on en connaît déjà plusieurs espèces auxquelles on a joint celles qui composent le genre Sissurelle de M. Dorbigny fils.

\section{G. CLI. Scalaire, scalaria, Lam.}

Anim. très spiral, muni d'une trompe, de deux tentacules terminés par un filet, et portant les yeux sur un renflement extérieur; pied court et ovale; organe excitateur mâle très grêle.

Coq. subturriculée, à tours de spire plus ou moins serrés, garnis de côtes longitudinales élevées, interrompues et presque tranchantes; ouverture ronde, assez petite, à bords réunis, et formant un. bourrelet mince et recourbé.

Operc. corné, mince, grossier et paucispiré,

Ce genre, dont l'animal est encore assez incomplétement: connu, contient plusieurs espèces vivantes et fossiles.

b) Ouverture munie d'un sinus à la réunion du bord exté rieur à la columelle.

G. CLII. Mélanopside, melanopsis, Fér. Et Pyrène, Lam.

Anim. muni d'un mufle proboscidiforme, avec deux tentacules contractiles, coniques, annclés, ayant 
chacun à leur base extérieure un pédoncule oculé; pied attaché au cou, très court, ovale, angulaire antérieurement de chaque côté; orifice respiratoire dans la gouttière formée par la réunion du manteau au corps.

Coq. épidermée, allongée, fusiforme ou conico-cylindrique, à sommet aigu; spire de six à quinze tours, le dernier formant souvent les deux tiers du test; ouverture ovale, oblongue; columelle torse, solide, calleuse, tronquée à sa base, séparée du bord extérieur par un sinus, la callosité se prolongeant sur la convexité de l'avant-dernier tour, formant une gouttière en arrière; quelquefois un sinus à la partie postérieure du bord droit. Operc. corné, subspiré, encore assez complet.

Le genre Mélanopside a été établi par M. de Férussac pour des coquilles d'cau douce, que leur columelle calleuse et sa troncature ne permettaient pas de placer avec les Mélanies; ce savant en a donné une monographie dans laquelle il établit les divisions suivantes :

$$
\text { rer groupe. }
$$

Un seul sinus au hord extérieur de l'ouverture, le séparant de la columelle. (G. Mélanopside, Lam.)

M. buccinoidea, etc.

$$
2^{\text {e groupe. }}
$$

Deux sinus distincts au bord extérieur de l'ouverture, l'un qui le sépare de la columelle, l'autre situé près de la réunion de ce bord avec l'avant-dernier tour. (G. Pyrène, Lam.)

$M$. atra, etc.

\section{G. CliII. Planaxe, planaxis, Lam.}

Anim. inconnu.

Coq. ovale conique, solide, sillonnée transversalement; ouverture oblongue; columelle aplatie et 
tronquée antérieurement, séparée du bord droit par un sinus; bord droit sillonné ou rayé en dedans, et épaissi par une callosité décurrente à son origine. Operc. corné, ovale, mince, subspiral.

M. de Lamarck a établi ce genre pour de petites coquilles fort voisines des Phasianelles, mais qui s'en distinguent cependant par la troncature de la partie antérieure de la columelle. Ce savant ne cite que les $P$. sulcata et undulata, mais nous en possédons six espèces bien distinctes ; nous avons eu occasion d'observer l'animal à l'lle-deFrance où les rochers en sont quelquefois couverts; mais, ayant perdu les notes que nous avions prises à son sujet, nous ne pouvons aujourd'hui donner ses principaux caractères; cependant nous croyons nous rappeler qu'il ne diffère que très peu de celui de la Phasianelle.

$$
\text { †† } 2^{\mathrm{e}} \text { prvision. }
$$

Un siphon plus ou moins prolongé pour conduire l'eau dans la cavité branchiale, et correspondant à une échancrure ou à un canal pratiqué à la partie antérieure de la coquille.

B. Presque tonjours un opercule; celui-ci non proportionné à la grandeur et à la forme de l'ouverture, souvent même comme rudimentaire.

\section{3e FAMILLE.}

\section{LES CÉRITES, Fér.}

\section{Canalifères, Lam.}

Anim. portant un mufle proboscidiforme, sans trompe, mais surmonté d'un voile; les tentacules oculés à leur partie moyenne et au-dehors.

Coq. allongée, à ouverture beaucoup plus petite que

le reste de sa longueur.

Operc. corné.

Marins. 
G. CLIV. Cértte, cerithium, Adans.

Et Potamide, Brong.

Anim. très allongé et spiral, muni d'un mufle proboscidiforme déprimé, recouvert par un voile souvent frangé ; tentacules distans, annelés, portant les yeux sur un renflement, à leur partie moyenne; bouche sans dent, mais avec une petite langue; pied court, ovale, avec un sillon marginal antérieur; manteau formant au côté gauche un canal ou commencement de siphon; cavité respiratrice ne renfermant qu'une seule branchie, longue et étroite.

Coq. turriculée, presque toujours tuberculeuse, à ouverture courte, ovale, oblique, offrant en avant un canal court, tronqué ou recourbé, et en arrière une gouttière.

Operc. corné, ovale, arrondi, subspiral et strié à sa face externe, enfoncé et rebordé à l'interne.

Ce genre très nombreux en espèces vivantes et fossiles, ne renferme que des animaux marins. Cependant il paraît que quelques uns d'eux vivent aux embouchures des fleuves, et ce sont précisément ceux-là dont M. Brongniart avait réuni les coquilles pour en former le genre Potamide qui ne peut être adopté en zoologie, ne reposant point sur des caractères assez tranchans. D'autres genres ont encore été faits aux dépens des Cérites, comme on peut le voir par la division suivante adoptée par M. de Blainville, et qui est très propre au classement des nombreuses espèces.

$$
\text { ser groupe. }
$$

Un petit canal fort court et recourbé obliquement vers le dos.

C. vertagus, etc.

$$
2^{\mathrm{e}} \text { groupe. }
$$

Un canal encore plus petit, mais tout droit; un sinus bien formé à la réunion postérieure des deux bords. (Les C. chenilles.)

C. aluco, etc. 


\section{3e groupe.}

Ouverture divisée en trois par la fermeture du tube court antérieur et celle du sinus postérieur. (G. Triphore ou Tristome, Deshayes.)

C. tristoma, etc.

$$
4^{\mathrm{e}} \text { groupe. }
$$

Un petit canal droit, dont les tours de spire sont plats et rubanés avec un ombilic profond; deux plis décurrens à la columelle, et un au bord droit. (G. Nériné, Defrance.)

C. nerinea, etc.

$$
5^{\mathrm{e}} \text { groupe. }
$$

Pas de canal, mais une simple échancrure, et dont le bord droit se dilate fortement avec l'âge. (G. Potamide, Brong.; Pyraze de Montf.)

C. palustre, etc.

M. de Blainville établit un sixième groupe, mais qui comprend le genre Pyrène que nous mettons avec les Mélanopsides à l'exemple de M. de Férussac.

La division qui renferme les Nérinés de $\mathbf{M}$. Defrance est peut-être douteuse, M. de Blainville pensant que ces coquilles pourraient être mieux près des Pyramidelles. Nous ne possédons en France que deux ou trois espèces de Cérites vivantes; mais nous en arons un très grand nombre à l'état fossile.

\section{$4^{\text {e FAMILLE. }}$}

LES POURPRES, Adans., Fér.

Canalifères, Ailées, Purpurifères, Columellaires, Lam.

Anim. non muni d'un voile, mais bien d'une trompe, ayant les tentacules oculés à leur partie moyenne, et en dehors.

Coq. de forme très variable, munie d'une échancrure ou plus généralement d'un canal droit ou recourbé. Operc. corné.

Marins. 
G. CLV. Bucain, buccinum, Adans.

Anim. muni de deux tentacules écartés, portant les yeux sur un renfiement extérieur, à leur partie moyenne; bouche sans dent labiale; pied court, arrondi en avant; manteau pourvu en avant de la cavité respiratrice d'un long canal toujours à découvert; organe de la respiration formé par deux peignes branchiaux inégaux; terminaison de l'oviducte au côté droit, à l'entrée de la cavité respiratrice; orifice du canal déférant à l'extrémité d'un appendice excitateur long, aplati, contractile, situé au côté droit du cou; anus au côté droit antérieur. Coq. ovale, allongée, à spire aiguë, mais médiocrement élevée; à ouverture oblongue ou ovale, très échancrée en avant; bord droit entier, quelquefois épais; columelle simple ou calleuse; rarement une columelle.

Operc. corné, ovale, à élémens subconcentriques; sommet peu marqué et marginal.

Le genre Buccin renferme un grand nombre de coquilles marines dont neuf ou dix espèces sont de nos côtes; il y en a aussi beaucoup de fossiles. M. de Férussac divise ce genre en deux sous-genres, comme il suit.

I $^{\text {er }}$ S.-G. Buccins proprement dits, Adans.

Anim. tel que dans la caractéristique du genre.

Coq. munie d'une columelle convexe, nue et sans ombilic.

Operc. comme dans la caractéristique du genre.

$B$. undatum, etc.

Il y en a de fossiles.

Les Alectrions font partie de ce sous-genre.

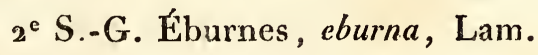

Anim. comme celui des Buccins proprement dits? 
Coq. toujours lisse et sans rides au bord; columelle largement et profondément ombiliquée.

$\boldsymbol{E}$. glabratum, etc.

In y en a de fossiles.

\section{G. CLVI. HARPe, harpa, Lam.}

Anim. muni d'une tête assez large, sans trompe, ayant la bouche ouverte en dessous; deux tentacules coniques, antérieurs et très rapprochés, portant les yeux sur un renflement situé un peu audessous de leur partie moyenne extérieure; pied grand, muni en avant d'une sorte de talon; siphon assez gros et un peu allongé ; peignes branchiaux inégaux, et au nombre de deux; orifice de l'oviducte à l'entrée de la cavité branchiale, du côté droit, celui du canal déférant ì l'extrémité d'un organe excitateur très volumineux; anus du même côté.

Coq. ovale oblongue, plus ou moins bombée, généralement assez mince, émaillée, munie de côtes longitudinales régulières; spire peu élevée et pointue, le dernier tour très grand; ouverture ovale, allongée, très largement échancrée en avant, le bord droit muni d'un bourrelet extérieur, la columelle simple, pointue en avant.

Operc. M. Reynaud, qui a étudié l'animal, n'en a point trouvé.

Ce geure, si remarquable par la beauté et la fraîcheur des coquilles qu'il renferme, appartient aux mers de l'Inde. On en compte cinq ou six espèces vivantes, et une seule fossile qui est des environs de Paris.

M. Reynaud, chirurgien-major de la corvette la Chevrette, a fait connaître tout récemment l'animal de la Harpe. Son travail ne nous a point donné lieu de changer la place que nous avions déjà adoptée pour ce mollusque par le seul fait de son analogie avec les genres voisins. MM. Quoy et Gaimard d'abord, et M. Reynaud ensuite, 
nous apprennent que l'animal de la Harpe peut quelquefois, lorsqu'il est attaqué par un ennemi, se débarrasser de la partie postérieure du pied, et rentrer complétement dans sa coquille, et M. Reynaud explique de la manière suivante cette particularité remarquable; il pense que la déchirure transversale qui cause, dans le mouvement de contraction de l'animal, la séparation de la partie postérieure de son pied vient de la résistance que cette partic trop volumineuse pour rentrer à la suise de l'animal éprouve sur les bords de la coquille. La Harpe ne paraît point avoir d'opercule, et cependant nous n'hésitons point à la laisser près des genres qui en sont pourvus, parce que d'abord elle a leur organisation, et qu'ensuite si elle est privée de cette pièce, elle a du moins la partie postérieure de son pied qui lui en tient en quelque sorte lieu.

\section{G. CLVII. Pourpre, purpura, Adans.}

Et Licorne, Montf.

Anim. muni d'une tête large, avec une trompe courte ou presque nulle; deux tentacules généralement en avant et rapprochés, de forme conique, et portant les yeux sur un renflement situé à leur partie moyenne extérieure; bouche inférieure presque toujours cachée par le pied, celui-ci assez grand, très avancé et comme bilobé en avant; manteau. formant un siphon distinct en avant ; peignes branchiaux au nombre de deux, inégaux; orifice de l'oviducte à l'entrée de la cavité branchiale, du côté droit, celui du canal déférant au côté droit du cou, à l'extrémité de l'organe excitateur, qui est généralement volumineux; anus du même côté.

Coq̣. ovale, épaisse, mutique ou tuberculeuse, à spire courte, le dernier tour plus grand que tous les autres ensemble; ouverture très dilatée, de forme ovale, terminée antérieurement par une échancrure oblique; columelle aplatie, finissant en pointe, en avant; bord droit tranchant, souvent épaissi 
et sillonné à l'intérieur, ou bien armé en avant d'une pointe conique.

Operc. corné, demi-circulaire, à sommet postérieur.

Ce genre renferme de belles espèces dont un très petit nombre est de nos côtes. Il y en a aussi à l'état fossile. On peut le partager en deux groupes de la manière suivante, car l'animal des Licornes ne diffère en rien de celui des Pourpres proprement dites.

${ }^{\mathrm{er}}$ groupe.

Le bord droit simple ou seulement sillonné en dedans. $P$. persica, eic.

$2^{e}$ groupe.

Le bord droit toujours très épais, et armé en avant d'une pointe conique.

P. imbricata, etc.

\section{G. CLVIII. Concholépas, concholepas, Lam.}

Anim. semblable à celui de la Pourpre, d'après M. Lesson.

Coc. épaisse, rude et sillonnée transversalement à la surface extérieure; spire très petite et à peine saillante; ouverture longitudinale, très ample, échancrée en avant, où se trouvent deux appendices dentiformes; point de columelle; les bords réunis; une impression musculaire très visible.

Operc. corné, transparent, trapezoïdal, à sommet marginal et élémens concentriques.

C'est à M. de Lamarck que l'on doit d'avoir placé les Concholépas à côté des Pourpres avec lesquelles nous aurions pu sans inconvénient les réunir à l'exemple de $M$. de Férussac. En effet, la communication que M. Lesson a bien voulu nous faire de l'animal, qu'il a rapporté de la mer du Sud, nous a prouvé qu'il ne diffère en rien de celui des Pourpres, son opercule seul montre dans sa forme un caractère bien tranché. On ne connait qu'une 
seule espèce de Concholépas, $C$. peruvianus, qui renferme deux variétés bien distinctes; elle est très abondante sur les côtes du Pérou et du Chili, et depuis quelques añées elle devient commune dans nos collections. On n'en connait point à l'état fossile.

\section{G. CLIX. NAsse, nassa, Lam.}

Anim. très déprimé, muni d'un pied fort grand, et dépassant le corps de toutes parts, mais surtout en avant, où il est large et auguleux, tandis qu'en arrière il se retrécit insensiblement. Du reste comme dans la caractéristique du genre Pourpre.

Coq. globuleuse, ovale ou subturriculée, à spire quelquefois très surbaissée ou assez élevée; ouverture oblongue, échancrée en avant, le bord droit tranchant, souvent plissé en dedans, le bord columellaire recouvert d'une large plaque calleuse 's'étendant plus ou moins loin.

Operc. corné.

Ce genre renferme plusieurs espèces vivantes, parmi lesquelles un très petit nombre est d'Europe. Il y en a plusieurs à l'état fossile.

\section{G. CLX. Tonne, dolium, Lam. Et Perdrix, Montf.}

Anim. semblable à celui de la Pourpre.

Coq. mince, presque globuleuse, ventrue, sillonnée transversalement; spire peu élevée, pointue, le dernier tour formant presque toute la coquille; ouverture grande, uvale, le bord droit ondulé; columelle souvent torse.

Operc. corné.

II. Cuvier forme deax groupes assez distincts parmi les espèces de ce genre; elles sont des mers de l'Inde, et deviennent quelquefois fort grosses. 
I $\mathrm{er}$ groupe.

Coq. globuleuse; la columelle torse. (Tonnes proprement dites.)

D. olearium, etc.

$2^{e}$ groupe.

Coq. oblongue; la columelle simple. (Perdrix, Montf.) D. perdix, etc.

G. ClXI. Cassidaine, cassidaria, Lam.

Anim. semblable à celui des Pourpres.

Coq. ovoïde, ventrue, à spire peu élevée; ouverture longue, un peu étroite, ovalaire, avec un canal antérieur peu recourbé; bord droit nuni d'un bourrelet; bord columellaire recouvert par une large callosité souvent granuleuse ou ridée. Operc. corné.

Ce genre ne renferme que peu d'espèces, dont une seule est des mers d'Europe. Il y en a aussi de fossiles.

\section{G. ClXiI. Casque, cassis, Brug.}

Anim. semblable à celui de la Pourpre.

Coq. ovalaire, bombée, à spire peu saillante, presque plane; ouverture oblique, longue et étroite, ayant un canal antérieur très court et recourbé vers le dos; bord droit épais, muni d'un bourrelet extérieur et denté en dedans; bord columellaire calleux, assez droit, et garni daris presque toute sa longueur de dents allongées et transverses. Operc. corné, très rudimentaire.

Ce genre renferme un assez grand nombre d'espèces vivantes ou fossiles. Parmi les premières, il y en a de très grandes et de fort belles, que l'on recherche beaucoup dans 
les collections. M. de Lamarck les divise de la manière suivante :

$$
l^{\mathrm{er}} \text { groupe. }
$$

Spire ayant des bourrelets.

C. cornuta, etc.

$$
3^{\mathrm{e}} \text { groupe. }
$$

Spire sans bourrelet.

C. rufa, etc.

G. CLXIII. Cancellaire, cancellaria, Lam.

Anim. semblable à celui de la Pourpre.

Coq. ovale ou globuleuse, réticulée, épaisse, à spire un peu plus élevée, pointue; ouverture demi-ovale, échancrée ou subcanaliculée en avant; bord droit tranchant, sillonné en dedans; columelle presque droite, avec plusieurs plis très saillans.

Operc. corné.

Plusieurs espèces du genre Cancellaire sont assez rares; il y en a un bon nombre à l'état fossile.

\section{G. CLXIV. Ricinule, ricinula, Lam.}

Anim. semblable à celui de la Pourpre.

Coq. ovale ou subglobuleuse, épaisse, hérissée de pointes ou de tubercules, à spire très courte; ouverture étroite, longue, offrant en avant une échancrure quelquefois subcanaliculée; le bord droit souvent digité en dehors et denté en dedans; le gauche calleux et muni de dents ou de plis.

Operc. corné, ovale, transverse, à élémens concentriques.

Ce genre renferme un petit nombre d'espèces, toutes 
exotiques, et agréablement variées par les belles couleurs de leur ouverture. On n'en connaît point de fossiles.

\section{G. CLXV. Rocher, murex, Lin.}

Anim. muni de deux tentacules longs et rapprochés; bouche sans mâchoire, mais armée de denticules crochus qui remplacent la langue; pied arrondi, généralement assez court; manteau grand, souvent orné de franges, au côté droit seulement; branchies formées de deux peignes inégaux; anus au côté droit, dans la cavité branchiale; orifice de l'oviducte au côté droit, à l'entrée de cette même cavité; orifice du canal déférant au bout de l'organe excitateur, au côté droit du cou.

Coq. ovale, oblongue, plus ou moins élevée du côté de la spire, ou prolongée en avant; la surface extérieure toujours interrompue par des rangées de varices en forme d'épines ou de ramifications, ou simplement de tubercules généralement dans un ordre régulier et constant; ouverture ovalaire, terminée antérieurement par un canal toujours droit, et plas ou moins allongé et fermé; bord droit souvent plissé ou ridé; bord columelle souvent calleux.

Operc. corné.

Le genre Rocher renferme un grand nombre de coquilles qui font l'ornement des collections. M. de Férussac n'y comprend point les Struthiolaires, que leur grande analogie avec les Tritons surtout nous a engagé à en rapprocher.

$I^{\text {er }}$ S.-G. Rocher, murex, Lam.

Anim. Voyez la caractéristique du genre.

Coq. ovale ou subglobuleuse, à spire généralement peu allongée, présentant à la surface extérieure trois bourrelets longitudinaux, ou davantage, plus ou moins chargés de varices; l'ouverture gé- 
néralement assez petite, parfaitement ovale; bord droit souvent chargé de dents ou de varices; bord columellaire recouvert par une lame unie; canal quelquefois très long et comme fermé.

Operc. corné, ovale, à cloisons subconcentriques, à sommet terminal.

Ce sous-genre, qui renferme des coquilles très-variées, a été singulièrement démembré par Denis de Montfort pour former plusieurs genres dont les caractères ne reposaient jamais que sur des différences de peu d'importance. Tout ce que l'on peut faire, c'est, à l'exemple de M. de Blainville, d'en former des groupes. Les Rochers sont de toutes les mers, et il y en a plusieurs fossiles.

$$
\text { I er groupe. }
$$

Un tube très long et épineux. (Les Bécasses.)

M. crassispina, etc.

$$
2^{e} \text { groupe. }
$$

Un tube très long non épineux. (Les Brontes.)

M. haustellum, etc.

$$
\text { 3e groupe. }
$$

Trois varices. (Les Triptères.)

$M$. acanthopterus, etc.

$$
4^{\mathrm{e}} \text { groupe. }
$$

Trois varices ramifiées. (Les Chicoracés.)

M. adustus, etc.

5e groupe.

Plus de trois varices ou bourrelets, le tube presque fermé.

M. melanomathos, etc.

$$
\text { 6e groupe. }
$$

Coquille subturriculée.

M. lyratus, etc. 


\section{$7^{\mathrm{e}}$ groupe.}

Coq. subturriculée; le tube fermé; un second tube à l'extrémité postérieure du côté droit se maintenant sur les tours de spire. (Typhis.)

$M$. pungens, etc.

$$
8^{e} \text { groupe. }
$$

Coq. plus globuleuse; la spire et le canal plus courts, très ouverts; l'ouverture subévasée.

M. vitulinus, etc.

$$
9^{\mathrm{e}} \text { groupe. }
$$

Un pli oblique à la columelle et un ombilic. (Phos.) M. senticosus, etc.

$$
\text { 2 } \text { S.-G. Ranelle, ranella, Lam. }
$$

Anim. inconnu.

Coq. ovale ou oblongue, déprimée, n'ayant que deux bourrelets ou rangées de varices situés latéralement; ouverture ovalaire; canal court, et un sinus à la réunion des deux bords, en arrière.

Operc. inconnu.

Il y en a plusieurs espèces fossiles.

$3^{\mathrm{e}}$ S.-G. Siruthiolaire, struthiolaria, Lam.

Anim. inconnu.

Coq. ovale, à spire assez élevée, à ouverture ovale, évasée; canal très court, très échancré; bord droit, sinueux, non denté, garni d'un bourrelet; bord columellaire calleux, étendu; un sinus à la réunion postérieure des deux bords.

Operc. corné.

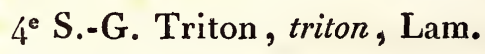

Anim. Voyez la caractéristique du genre.

Coq. ovale, à spire généralement assez élevée, revêtue à sa face extérieure de bourrelets ou varices irrégulièrement épars, et ne formant jamais 
de rangées longitudinales; ouverture ovale ou oblongue, avec un canal antérieur droit et médiocre; bord droit souvent chargé de plis ou de denticules; bord columellaire tapissé par une callosité souvent très ridée.

Operc. corné, ovale, assez grand.

G. CLXVI. Colombelle, colombella, Lam.

Anim. présentant les caractères de la famille, mais du reste incomplétement connu.

Coq. épaisse, ovale, turbinée, à spire courte; ouverture longue, étroite, échancrée en avant; columelle un peu plissée; bord droit renflé au milieu de son côté interne.

Operc. corné, elliptique.

Ce genre renferme quelques espèces fossiles.

\section{G. CLXVII. Tukbinelee, turbinella, Lam.}

Anim. très imparfaitement connu.

Coq. turbinée ou turriculée, épaisse, à spire quelquefois surbaissée et mamelonée au sommet; ouverture ovale, avec un canal toujours fort droit et le plus souvent allongé; bord droit tranchant, bord columellaire souvent calleux, et portant plusieurs plis comprimés et transverses.

Operc. inconnu.

Le genre Turbinelle renferme une trentaine d'espèces dont plusieurs se confondraient facilement, les unes avec les Fuseaux, les autres avec les Pyrules, si les plis de la columelle ne les en distinguaient pas. Il y en a de fossiles.

\section{G. CLXVIII. Pyrule, pyrula, Lam.}

Anim. inconnu.

Coq. piriforme, ventrue, à spire généralement peu élevée ou surbaissée; canal toujours droit, souvent plus long et atténué à son extrémité, quel- 
quefois aussi très court et large; ouverture ovale, plus ou moins allongée, et assez grande; bord droit tranchant; columelle lisse, arquée. Operc. corné.

Les Pyrules sont assez nombreuses et communes; toutes sont exotiques; cependant nous avons vu deux individus de la $P$. melongena venant de la Teste où ils avaient été ramassés sur le sable. Il y a plusieurs jolies espèces de Pyrule à l'état fossile.

\section{G. CLXIX. Fasciolaire, fasciolaria, Lam.}

Anim. inconnu.

Coq. fusiforme, peu épaisse, assez renflée dans son milieu, à spire médiocre; ouverture ovale; canal assez long, quelquefois un peu arqué; bord droit tranchant, souvent ridé en dedans; bord columellaire offrant quelques plis très obliques. Operc. inconnu.

M. de Lamarck cite plusieurs espèces de Fasciolaires, comme appartenant à la mer des Indes. Nous n'en avons jamais rencontré que dans celle des Antilles, et les collections de l'Ile-de-France que nous avons visitées ne nous en ont offert aucune. Il y en a de fossiles.

\section{G. CLXX. Fuseau, fusus, Lam.}

Anim. inconnu, mais ne paraissant pas devoir beaucoup différer de celui des rochers.

Coq. fusiforme, souvent ventrue dans son milieu, rugueuse, épaisse, à spire très élevée; canal très droit et allongé; ouverture ovale; bord droit tranchant, le gauche lisse.

Operc. corné.

Le genre Fuseau est remarquable par les belles coquilles qu'il renferme; mais son animal n'est qu'imparfaitement connu et seulement par quelques figures d'Adanson. Il ne 
comprend que des coquilles marines qui présentent un grand nombre d'espèces tant à l'état frais qu'à l'état fossile.

\section{G. CLXXI. Pleurotome, pleurotoma, Lam.}

Coq. fusiforme ou turriculée, avec un canal droit souvent assez allongé; ouverture ovale, le bord columellaire simple et lisse, le bord droit tranchant, muni d'une entaille.

Operc. corné.

Nous pensons que l'on doit rapporter aux Pleurotomes le genre Defrancie de M. Millet, établi pour des coquilles fossiles dont le bord droit se replie singulièrement sur l'ouverture. Ce serait un troisième groupe.

Ce genre peut se diviser ainsi.

I $^{\text {er }}$ groupe. Les Pleurotomes proprement dits.

Une scissure étroite au bord droit.

$P$. muricata, etc.

$2^{\circ}$ groupe. Ies Clavatules.

Une entaille très large au bord droit près de la spire. $P$. imperialis, etc.

Il y en a plusieurs à l'état fossile.

G. CLXXII. Rostellaire, rostellaria, Lam. Et Hippocrène, Montf.

Anim. imparfaitement connu, mais assez semblable à celui du murex, d'après M. Cuvier.

Coq. fusiforme ou subturriculée, à spire élevée, pointue; canal saillant et en bec pointu; ouverture ovalaire; bord droit simple, denté, digité ou très dilaté, et muni d'un sinus voisin du canal. Operc. corné et très petit.

Le genre Hippocrène de Montfort comprend les Rostellaires dont le bord droit très dilaté est simple. 
5 e FAMILLE.

LES STROMBES, Fér.

Anin. non muni d'un voile, mais bien d'une trompe, ayant les yeux portés sur des pédicules latéraux plus grands que les tentacules mêmes.

Coq. ailée, à ouverture presque aussi longue qu'elle, et un peu étroite.

Operc. corné.

Marins.

G. CLXXIII. Strombe, strombus, Lin. Et Ptérocère, Lam.

Anim. spiral, un peu comprimé, muni d'une trompe à l'extrémité de laquelle se trouve la bouche qui est fendue en long; celle-ci contenant un ruban lingual, garni d'aiguillons recourbés en arrière; tentacules cylindriques, obtus et courts; yeux portés sur deux pédoncules, cylindriques et gros, plus longs que les tentacules, et placés à leur côté extérieur; pied assez petit et élargi en avant; manteau formant en avant un canal généralement assez court; orifices de l'anus et de l'oviducte en arrière.

Coq. épaisse, ovale oblongue, subinvolvée, conique en avant comme en arrière, à spire médiocrement élevée; ouverture longue et étroite, terminée antérieurement par un canal plus ou moins long ou recourbé; bord droit dilaté et offrant un sinus un peu en arrière du canal; bord columellaire simple, mais quelquefois calleux.

Operc. corné, long et étroit, à sommet terminal, à élémens comme imbriqués.

La considération de la coquille des Strombes permet d'établir entre eux une division de second ordre.

$I^{\text {er }}$ S.-G. Strombes, strombus, Lin.

Anim. Voyez la caractéristique du genre. 
Coq. munie d'une aile simple, et d'un canal très court, tronqué ou échancré.

Operc. Voyez la caractéristique du genre.

Les Strombes sont très nombreux et de toutes les mers. Il y en a aussi de fossiles.

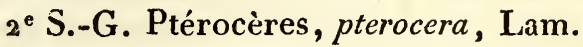

Anim. Voyez la caractéristique du genre.

Coq. munie d'une aile digitée, et en avant d'un canal allongé.

Operc. Voyez la caractéristique du genre.

Les Ptérocères sont moins nombreux et tous des mers de l'Inde. M. Dorbigny fils en a trouvé des moules fossiles dans les terrains de La Rochelle.

\section{6e FA MiLLE.}

\section{LES CONES, Fér.}

Anim. non muni d'un voile, mais bien d'une trompe, ayant les tentacules oculés à leur sommet ou vers leur sommet.

Coq. variable dans sa forme, mais toujours en cône plus ou moins allongé, soit que le sommet de ce cône se trouve au sommet de la spire, soit qu'il occupe l'extrémité antérieure de la coquille.

Operc. corné.

Marins.

a. Tentacules cylindriques oculés près de leur sommet.

G. CLXXIV. Cône, conus, Lin.

Et Rhombe, Rouleau, Cylindre, Hermès, Montf.

Anim. allongé, fort comprimé et involvé, muni d'une tête très distincte, terminée par une trompe susceptible de beaucoup d'extension; bouche con. tenant une langue assez courte quoique saillante, et hérissée de deux rangées de dents aiguës; ten- 
tacules cylindriques portant les yeux près de leur sommet; pied ovale, assez allongé, plus large en avant qu'en arrière, avec un sillon transverse antérieur; manteau étroit, formant en avant un siphon allongé.

Coq. épaisse, solide, involvée, en forme de cône; spire peu ou point élevée; ouverture longue et très étroite, versante en avant; bords droits et parallèles, l'extérieur simple et tranchant, le gauche muni de quelques plis seulement en avant. Operc. corné, très petit, subspiré, à sommet terminal placé obliquement sur l'arrière du pied.

Ce genre est le plus beau, le plus étendu et le plus intéressant, dit M. Lamarck; en effet la monographie qu'il en donne renferme cent quatre-vingt-une espèces vivantes (1), parmi lesquelles on remarque les coquilles les plus précieuses. Elles sont toutes, à l'exception de trois espèces de la Méditerranée, des mers équatoriales. Il y en a aussi plnsieurs fossiles en France.

Les genres Rhombe, Cylindre, Rouleau et Hermès, qui ont été établis aux dépens des Cônes par Montfort, peuvent servir à former des groupes parmi les espèces. M. de Lamarck ne les divise qu'en deux sections, les Cônes couronnés, comme les Rhombes et les Cônes, et les non courronnés qui comprennent toutes les autres espèces.

b. Tentacules triangulaires oculés au sommet.

\section{G. CLXXV. Alène, subula, Blainv.}

Anim. très élevé, portant des tentacules extrêmement petits et triangulaires, avec les yeux au sommet; une longue trompe labiale sans crochets, au fond

(I) M. Duclos, qui s'occupe d'une nouvelle monographie de ce genre, s'est assuré que, sur ce nombre, il y en a plusieurs qui ne doivent fournir que des variétés tout au plus. 
de laquelle est la bouche; pied très court, rond. Coq. non épidermée, turriculée, à spire pointue; tours de spire lisses, rubanés, bifides; ouverture ovale, petite, largement échancrée en avant; bord droit mince, tranchant ; bord columellaire chargé d'un bourrelet oblique à son extrémité.

Operc. corné, ovale, à élémens lamelleux, comme imbriqués.

M. de Blainville a établi le genre Alène sur la connaissance de l'animal de la Vis tachetée, rapporté en France par MM. Quoy et Gaimard; il réunit en conséquence à ce genre toutes les espèces dont la coquille est très élevée, la spire pointue, les tours rubanés, et par conséquent le plus grand nombre des espèces décrites par M. de Lamarck. Elles sont presque toutes des mers de l'Inde et de l'Australasie. Il rapporte également à ce genre plusicurs espèces fossiles qui avaient été rangées parmi les Vis. Il est vivemeut à désirer que de nouvelles observations sur les animaux de ces coquilles viennent séparer décidément les espèces qui doivent appartenir à chacun des deux genres.

C. Très rarement un opercule. Celui-ci, quand il existe, rudimentaire.

\section{$7^{\text {C FAMILLE. }}$}

LES ENROULÉS, Lam.

Anim. muni de tentacules conico-subulés, portant les yeux au côté extérieur, tout près de leur base ou à leur partie moyenne.

Coq. oblongue, plus ou moins allongée, à ouverture souvent étroite, et plus ou moins échancrée.

Operc. dans un seul genre encore; corné. Marins.

G. CLXXVI. Vis, terebra, Brug.

Anim. spiral ; tête bordée d'une petite frange, et munie de deux tentacules cylindriques terminés en pointe et peu distans; yeux à la base externe; 
bouche sans trompe; pied ovale, avec un sillon transversal antérieur et deux auricules latérales; siphon très allongé.

Coq. non épidermée, ovalaire, à spire aiguë assez peu élevée ou subturriculée; ouverture large, ovale, fortement échancrée en avant, le côté droit simple, la columelle chargée d'un bourrelet oblique à son extrémité.

Ce genre, d'après M. de Lamarck, est assez étendu, puisqu'il comprend vingt-quatre espèces; mais des observations récentes faites par $M$. de Blainville sur l'animal de la $T$. maculata, ont fait connaître à ce naturaliste qu'il fallait séparer du genre cette espèce, et peut-être toutes celles qui, comme elle, sont très élevées pour en former un genre à part, et en effet tellement distinct par les caractères de l'animal qu'il se refuse même à entrer dans la même famille. Nous avons provisoirement adopté cette nouvelle manière de voir, fondée sur une observation dont on ne pent douter, mais qu'il serait essentiel d'étendre à d'autres espèces de Vis, afin de connaitre les limites de ces deux genres si rapprochés par les caractères de leurs enveloppes testacées. Ainsi donc le genre Vis de M. de Blainville ne comprend plus que les espèces qui, par leur forme générale, ont quelques ressemblances avec les Buccins; le Miran d'Adanson en est le type. Il y en a de fossiles.

\section{G. CLXXVII. Mitre, mitra, Lam. Et Minaret, Montf.}

Anim. inconnu.

Coq. turriculée ou subfusiforme, à spire pointue; ouverture généralement petite, étroite, triangulaire et échancrée en avant; bord droit tranchant, plus long que le bord columellaire; celui-ci recouvert d'une lame mince chargée de plis obliques et parallèles, dont les antérieurs sont les plus petits.

Le genre Mitre est un des plus beaux, et se compose de plus de quatre-vingts espèces vivantes, la plupart des mers Australes et de l'Inde; il y en a aussi un assez bon nombre 
à l'état fossile. Montfort désignait sous le nom générique de Minarets, celles qui, très allongées et côtelées, n'offrent qu'un pli à l'ouverture.

G. CLXXVIII. Taris̀ne, terebellum, Lam. Et Séraphe, Montf.

Anim inconnu.

Coq. mince, polie, subcylindrique, très enroulée, à sommet pointu; ouverture longitudinale, triangulaire, très étroite en arrière et échancrée en avant; bord droit simple et tranchant; bord columellaire lisse, tronqué et un peu prolongé en avant.

Ce genre ne renferme qu'une espèce vivante, le $T$. subulatum, qui est de la mer des Indes; les autres espèces connues sont fossiles. Montfort comprenait sous le nom de Séraphe les espèces dans lesquelles le bord droit se prolonge jusqu'au sommet, et où, par conséquent, l'ouverture est aussi longue que la coquille. Le $T$. convolutum, fossile de Grignon, est le type de son genre qui ne serait tout au plus propre qu'à former un groupe.

G. CLXXIX. Ancrldarne, ancillaria, Lam. Ancille, Montf.; Anaulace, de Roiss.

Anim. muni d'un lobe du manteau recouvrant la coquille, du reste inconnu.

Coq. lisse, oblongue, subcylindrique, à spire pointue, médiocrement élevée, et à sutures non canaliculées; ouverture ovale, allongée, tronquée antérieurement avec une large échancrure, anguleuse postérieurement; bord droit simple; columelle chargée ell avant d'un bourrelet calleux et oblique.

Les Ancillaires ne renferment qu'un petit nombre d'espèces. M. de Lamarck en cite quatre à l'état vivant; l'une d'elles, l' $A$. cinnamomea, est très rare, et ressemble à de jeunes Porcelaines telles que celles qui avaient donné lieu, mais mal à propos, à former le genre Péribole. Il y a plusieurs Ancillaires fossiles. 


\section{G. CLXXX. Onive, oliva, Brug.}

Anim. involvé, comprimé, muni d'une petite tête terminée par une trompe; tentacules rapprochés, élargis à leur base, et subulés à leur extrémité, portant les yeux sur de petits renflemens, à leur partie moyenne extérieure ; pied très grand, oblong et fendu transversalement en avant; manteau avec un seul lobe latéral recouvrant en grande partie la coquille, offrant deux languettes au côté de l'ouverture branchiale, et formant en avant un siphon très allongé; un seul peigne branchial; organe mâle très volumineux, au cốté droit antérieur. Coq. épaisse, solide, polic, subcylindrique, à spire peu élevée, les tours séparés par un canal; ouverture longue, étroite, échancrée en avant; bord droit simple; bord columellaire épaissi et strié obliquement dans presque toute sa longueur, une callosité sailiante à sa partie postérieure. Operc. corné, allongé, très petit, à sommet marginal.

Ce genre fait partie du petit nombre de ceux qui prouvent qu'on ne peut se servir de l'opercule pour diviser les Pectinibranches en Pomastomes et Apomastomes; car s'il fallait par égard pour la présence d'un opercule dans les Olives les porter dans la première de ces divisions, il en résulterait que l'on éloignerait l'un de l'autre des mollusques qui semblent ve pouvoir être séparés, tant par leur propre organisation que par les caractères généraux de leur coquille. C'est, ainsi que nous avons vu, que, malgré l'absence de l'opercule, il était nécessaire de conserver près des Buccins les Harpes, et près de la Phasianelle le Litiope.

Le genre Olive est un des plus riches et des plus recherchés par la beauté et la diversité des espèces; M. Duclos en a fait une étude particulière sur la belle collection qu'il a rassemblée à ce sujet, et les conchyliologistes attendent avec impatience la publication d'un travail qui, quoique fait en 1824 , n'a point encore paru. Ce naturaliste ayant 


\section{MÉTHODIQUE, etc.}

bien voulu nous permettre de l'examiner, nous avons pu juger de son importance, et voici, entre autres, ce que nous y avons trouvé de remarquable. Environ quarante espèces nouvelles viennent enrichir le genre; mais d'un autre côté plusieurs de celles de M. Lamarck disparaissent, parce qu'elles n'avaient été établies que sur des variétés d'âge, car M. Duclos reconnaît qu'une même espèce varie du blanc au noir ou d'une couleur uniforme à une couleur fasciée, et il le prouve en passant de l'une de ces variétés extrêmes à l'autre par une transition insensible, et souvent par des individus mixtes qui, à la rigueur, suffiraient pour lever tous les doutes. Enfin M. Duclos, ayant sous les yeux un grand nombre d'espèces, a reconnu parmi elles des formes principales dont il s'est servi pour établir les groupes suivans.

$$
1^{\text {er }} \text { groupe. Les Ancylloïdes. }
$$

Qui ont quelques rapports de forme avec les Ancyllaires, et dont les plis columellaires sont en forme de torsade.

o. hyatuia, etc.

$$
2^{\mathrm{e}} \text { groupe. Les Cylindroïdes. }
$$

Qui sont cylindracées, à spire fort pointue avec des plis columellaires très nombreux occupant presque tout le bord gauche.

o. subulata, etc.

$3^{\text {e }}$ groupe. Les Glandiformes.

Qui sont globuleuses, ventrues, à spire très courte. o. porphyra, etc.

$4^{\mathrm{e}}$ groupe. Les Volutelles.

Dont la spire est mucronée, et dont le canal s'oblitère vers le commencement du dernier tour.

o. brasiliana, etc.

La dernière espèce de ce groupe, l' $O$. auricularia, conduit aux Volutes par sa spire mucronée, le développement de 
son bord droit, et l'absence presque totale de ses plis columellaires.

On connaît plusieurs espèces d'Olives à l'état fossile.

\section{G. CLXXXI. Pokcelaine, cypraca, Lin. Et Péribole, Adans.}

Anim. allongé, muni de deux tentacules coniques assez longs, portant les yeux à leur base extérieure, sur de petits renflemens; bouche verticale dans le fond d'une petite cavité, et contenant un ruban lingual hérissé de tentacules et prolongé dans l'abdomen; pied ovale, allongé, assez grand; mantean formant deux lobes latéraux très grands, pouvant se croiser sur le dos de la coquille; siphon très court et formé dans la ligne médiane par les bords des deux lobes; anus à l'extrémité d'un tube, en arrière de la cavité branchiale; organe mâle beaucoup plus en avant, et communiquant avec l'orifice du canal déférant par un sillon.

Coq. très polie, ovale ou ovale-oblongue, plus ou moins bombée ou cylindracée, à spire extrêmément petite et empatée; ouverture longitudinale, étroite, dentée de chaque côté, versante aux deux extrémités, où elle est comme échancrée et à bords roulés en dedans.

Le genre Porcelaine est sans contredit un des plus beaux par le nombre des espèces qu'il renferme, la variété de leurs formes et la richesse de leurs couleurs; aussi est-ce un de ceux que l'ou s'empresse le plus de compléter dans les collections. Il en est un grand nombre d'espèces fort communes; mais il en est aussi quelques unes de très rares et d'un prix élevé. Lorsque ces coquilles sont jeunes, elles présentent une disposition de formes et des couleurs toutes différentes de celles qu'elles doivent avoir à l'âge adulte; par exemple, leur bord droit, au lieu d'être retourné au-dedans, est simple et tranchant, ce qui, trompant Adanson, l'avait conduit à former un genre à part sous le nom de Péribole, que M. de Blainville, d'après 
son autorité, avait cru devoir conserver. Les animaux de ces coquilles les recouvrent des vastes lobes de leurs manteaux, les rendent extrêmement lisses, et en y déposant le principe qui les colore, y impriment vers le milieu du dos une ligne assez remarquable dans certaines espèces, et qui répond à la ligne de jonction de ces mêmes lobes. Ces mollusques, presque tous des mers chaudes, habitent sous les rochers et les pierres. C'est, sans doute, par inadvertance que M. de Lamarck a avancé qu'ils pouvaient à un certain âge quitter leur coquille pour en construire de nouvelle, et il est fâcheux de voir dans des ouvrages plus récens propager de semblables erreurs.

M. Duclos, dont nous venons de citer le travail sur les Olives, s'est également occupé, et avec le même succès, des Porcelaines. Il a pu, en rassemblant sous ses yeux un très grand nombre d'individus de tous les âges, et les comparant ensemble, rectifier les doubles emplois d'espèces qui avaient été faits, et préciser dans chacune d'elles lès différens états par lesquels elles passent avant d'arriver à celui d'adulte. Par ses recherches, le genre acquiert également une quarantaine d'espèces de plus.

Ce naturaliste forme les trois groupes suivans :

I er groupe. Les Globuleuses.

Qui comprennent toutes les espèces dont le dos est complétement lisse.

C. tigris, etc.

$2^{\circ}$ groupe. Les Pustuleuses.

Celles dont le dos est chargé de points ronds, élevés en forme de verrues.

C. nucleus, etc.

3e groupe. Les Striées.

Celles qui ont des stries transversales se contiuuant sur les côtés, et remontant sur le dos jusqu'au sillon dorsal.

C. oniscus, etc. 
Ce genre renferme plusieurs espèces fossiles.

\section{G. CLXXXII. Ovuris, ovula, Lam. Et Calpurne, Ultime, Navette, Montf.}

Anim. semblable à celui des Porcelaines.

Coq. oblongue, bombée, atténuée et quelquefois acuminée aux deux bouts, et sans spire; ouverture longue, étroite et échancrée aux deux extrémités; les deux bords roulés en dedans, le droit quelquefois ridé ou denté, le gauche jamais.

Ce genre renferme des coquilles très voisines des Porcelaines, et que l'on recherche beaucoup dans les collections; on en connaît une douzaine à l'état vivant et plusieurs à l'état fossile. La plupart sont de la mer des Indes et de celles d'Amboine ou de Chine; il y en a aussi dans la Méditerranée et la mer Noire. Quelques dispositions particulières dans le bord de leur ouverture ou dans le prolongement de leurs extrémités avaient conduit Montfort à les diviser en plusicurs genres; mais on ne peut se servir de ces divisions que pour former des groupes.

$$
\text { rer groupe. Les Orules. }
$$

Coq. très bombée; le côté droit denté, les extrémités peu proéminentes.

O. oviformis, etc.

$$
2^{e} \text { groupe. Les Ultimes. }
$$

Coq. munie d'un pli obtus transversal sur le dos; les bords de l'ouverture non dentés.

O. gibbosa, etc.

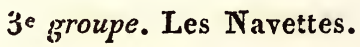

Coq. allongée, acuminée à chaque extrémité; les bords de l'ouverture non dentés.

O. volva, etc.

Il y en a à l'état fossile. 
G. CLXXXIII. Volvaire, volvaria, Lam.

Anim. inconnu.

Coq. cylindracée, très involvée, à spire non saillante; ouverture étroite, aussi longue qu'elle; bord droit mince et tranchant; columelle offrant plusieurs plis à sa partie antérieure.

M. de Blainville confond les Volvaires avec les Marginelles; les caractères qui distinguent ces coquilles nous ont engagé à conserver les premières comme genre, puisque nous ne connaissons pas encore leur animal; quoi qu'il en soit, on ne peut disconvenir qu'elles ne soient très voisines; on serait même tenté de croire que les Volvaires sont de jeunes Marginelles. Il y a des Volvaires fossiles.

\section{G. CLXXXIV. Margineler, marginella, Lam.}

Porcellana, Adans.

Anim. comprimé, muni d'une tête très distincte, avec une petite trompe; bouche contenant un ruban lingual hérissé de denticules, et se prolongeant dans la cavité viscérale; tentacules coniques, portant les yeux à leur base extérieure, sur de petits renflemens; pied elliptique, très grand ; manteau offrant de chaque côté un lobe qui peut se réfléchir vers le dos de la coquille, et en avant un siphon assez allongé.

Coq. polie, ovale oblongue, à spire courte et mamelonnée; ouverture étroite, allongée, ovalaire, à peine échancrée en avant; bord droit garni d'un bourrelet en dehors; bord columellaire garni de plis obliques presque égaux.

M. de Lamarck divise ainsi les espèces de ce genre :

$$
\text { I er groupe. }
$$

Espèces à spire saillante.

M. glabella, etc. 
$2^{e}$ groupe.

Espèces à spire non saillante.

M. dactylus, etc.

Il y en a plusieurs à l'état fossile.

8e Famille.

LES VOLUTES, Fér.

Columellaires, Lam.

Anim. muni de tentacules triangulaires et aplatis, portant les yeux en arrière sur le côté externe.

Coq. oblongue, à ouverture grande, et munie de plis à la columelle.

Marins.

G. CLXXXV. Volute, voluta, Lin.

Anim. ovale; la tête distincte, munie de tentacules triangulaires et assez courts, avec des yeux sessiles à leur base, un peu en arrière; bouche à l'extrémité d'une trompe épaisse, garnie de dents en crochets; pied fort large et débordant de toutes parts la coquille, se ployant longitudinalement pour $y$ rentrer; branchies composées de deux peignes; anus sessile.

Coq. ovale, un peu ventrue, à spire peu élevée et mamelonnée; ouverture grande, plus longue que large, échancrée en avant; bord droit arqué; columelle excavée, garnie de plis obliques, dont les antérieurs sont les plus grands.

Ce genre, ainsi que le suivant, qui devrait peut-être lư être réuni, sont remarquables par la grandeur et la beauté des espèces qu'ils renferment. M. de Lamarck en caractérise trente vivantes; mais nous en avons vu plusieurs nouvelles dans la belle collection de M. le duc de Rivoli. Ce sont génèralement des coquilles de l'Australasie et de la mer des Indes. Il y en a un bon nombre à l'état fos- 


\section{MÉTHODIQUE, etc.}

sile, et qui ont une grandeur beaucoup moindre que celle des espèces vivantes. Pour suivre l'exemple de M. de Lamarck, nous pourons le diviser ainsi :

rer groupe. Muricines.

Coq. ovale, épineuse ou tuberculeuse.

$V$. imperialis, etc.

$\mathbf{2}^{\mathrm{e}}$ groupe. Musicales.

Coq. ovale, subtuberculeuse.

$V$. hebraea, etc.

$3^{\text {e }}$ groupe. Fusoïdes.

Coq. allongée, ventrue, presque en fuseau.

$V$. magnifica, etc.

G. CLXXXVI. Yex, cymbium, Adans.

Anim. oval très renflé, pouvant à peine rentrer dans sa coquille, et la débordant de toutes parts avec son pied, qui est très grand; tête munie d'un voile $d$ 'où sortent deux tentacules triangulaires et aplatis; yeux à la base extérieure de ceux-ci, un peu en dehors; une trompe avancée avec la bouche à son extrémité.

Coq. oblongue ou ovale, très ventrue, assez mince; spire généralement courte, mamelonnée; ouverture très grande, plus longue que large, échancrée en avant; bord droit arqué, tranchant; bord gauche ou columellaire excavé, murii un peu en avant de son milieu, de quelques plis obliques.

Ce genre est très voisin du précédent; cependant nous n'avons point trouvé de voile à l'animal des Volutes, et celui des Yets en est évidemment muni. Cette seule considération nous a engagé à les séparer; car si les animaux étaient les mêmes, les Yets ne devraient former tout au plus qu'un sous-genre des Volutes. Ce sont de très grandes co- 
quilles, toutes exotiques et la plupart des pays chauds. M. de Lamarck en compte quatorze espèces qui, selon nous, doivent être plutôt réduites qu'augmentées, les jeunes individus offrant quelquefois dans la coquille des caractères qui disparaissent avec l'âge.

\section{†† $3^{\mathrm{e}}$ orvision.}

Point d'appendices membraneux latéraux, ni de siphon pour conduire l'eau aux branchies, la coquille étant généralement trop petite pour l'animal; bouche sans dents, mais pourvue inférieurement d'un long ruban lingual.

$$
9^{\text {e FAMIILE. }}
$$

LES SIGARETS, Fér.

Anim. portant des tentacules oculés à leur base externe.

Coq. nulle, externe ou interne, en forme d'oreille, très déprimée et à ouverture fort grande. Marins.

\section{G. CLXXXVII. Cormocelne, coriocella, Blainv.}

Anim. elliptique, fort déprimé, ayant les bords du manteau très minces, échancrés en avant, débordant largement de toutes parts; pied ovale, très petit; tête peu distincte; deux tentacules cachés sous le bouclier, assez gros, courts, contractiles; yeux à leur base externe; le dos peu bombé. Coq. nulle.

Nous empruntons la caractéristique de ce genre à M. de Blainville. La Coriocelle est un mollusque de l'Ile-deFrance.

\section{G. CLXXXVIII. Sigaret, Sigaretus, Lam.}

Anim. oblong, très bombé, plane en dessous, recouvert d'un manteau dépassant de toutes parts, et échancré en avant; tête large, munie de deus 
tentacules coniques, oculés à leur base extérieure ; pied très grand; branchies composées de deux peignes; organe de la génération et orifice de l'anus du côté droit antérieur, la verge du mâle étant très grande.

Coq. interne plus ou moins épaisse, très déprimée, à spire aplatie, latérale; ouverture entière, très grande, oblongue, à bords désunis, le droit mince et tranchant, le gauche replié, court et en spirale; deux impressions musculaires latérales.

Ce genre renferme un petit nombre d'espèces, parmi lesquelles quelques unes sont très incomplétement caractérisées; il en est qui sont solides et épaisses; d'autres, au contraire, sont minces, transparentes et comme membraneuses. Peut-être si leurs animaux étaient mieux connus, fourniraient-ils des divisions importantes parmi elles. On en connaît à l'état fossile.

\section{G. CI_XXXIX. CRуртоsтome, cryptostomus, Blainv.}

Anim. glossoïde, formé en très grande partie par un pied fort long, très épais, plus étroit en avant, canaliculé de chaque côté, en débordant beaucoup. de toutes parts la masse tortillée des viscères, qui est fort petite, peu convexe en dessus, et recouverte dans son tiers médian par la coquille; bouche très petite, cachée sous le rebord antérieur et supérieur du pied, vers laquelle convergent ses quatre sillons; deux tentacules comprimés et appendiculés à leur base; yeux? un seul grand peigne branchial; anus au côté droit du bord libre du manteau. Coq. interne, en tout semblable à celle des Sigarets.

Nous ne connaissons pas ce genre dont nous empruntons la caractéristique à $M$. de Blainville; ce naturaliste annonce deux espèces, pensant que quelques Sigarets de M. de Lamarck pourraient peut-être renir s'y ranger. 
G. CXC. Stomatelle, stomalella, Lam. Et Padole, Montf.

Anim. inconnu.

Coq. nacrée à l'intérieur, très déprimée, orbiculaire ou oblongue, auriforme; ouverture très grande, entière, plus longue que large, le bord droit évasé, dilaté, ouvert, le gauche plus épais et un peu aplati.

Ce genre, très peu connu, renferme, d'après M. Lamarck, quatre ou cinq espèces, toutes des mers de l'Inde.

G. CXCI. Sтomate, stomatia, Lam.

Anim. inconnu.

Coq. nacrée à l'intérieur, arrondie, auriforme, déprimée, à spire proéminente; ouverture entière, ample, un peu plus longue que large, le bord droit mince et tranchant, aussi élevé que le bord columellaire; une côte transversale et tuberculeuse sur le dos.

Ce genre, ainsi que les précédens, mérite de nouvelles recherches et de soigneuses comparaisons. Quelques auteurs, M. de Blainville entre autres, laissent les Stomates avec les Haliotides. Elles sont des mers de l'Inde.

G. CXCII. Velutre, velutina, Blainv.

Anim. ovale, assez bombé, à peine spiral; bord du manteau simple en avant, et double dans toute sa circonférence; lèvre interne plus épaisse et tentaculaire; pied petit, ovale, avec un sillon marginal antérieur; tête épaisse; tentacules gros, obconiques, distans, avec un petit voile frontal entre eux; yeux noirs, sessiles au côté externe de la base de ces tentacules; bouche grande, à l'extrémité d'une sorte de mufle; cavité respiratrice grande, sans trace de tube, et contenant deux peignes branchiaux 
inégaux, obliques, attachés au plancher; orifice de l'ovaire à la base de l'organe excitateur mâle, situé à la racine du tentacule droit; attache musculaire en fer à cheval, fort mince en arrière, ouvert en avant.

Coq. extérieure, épidermée, patelliforme, à spire petite, latérale, sans columelle; ouverture grande, à bords presque réunis, l'un et l'autre tranchans, le droit se réunissant au gauche par un dépôt calcaire lamelleux.

Nous empruntons la caractéristique de ce genre intéressant à M. de Blainville, qui l'a établi pour un mollusque des côtes d'Angleterre que MM. Audouin et Milns Edwards ont retrouvé sur les côtes de France.

$$
\delta^{\text {e }} \text { ORDRE. }
$$

\section{SCUTIBRANCHES, Cuv.}

Et Cervicohranches, Chismobranches, Blainv.; Gast.calyptraciens et Trachélipodes néritacés, Lam.

Anim. muni d'un pied pour ramper; des branchies pour respirer, sous forme de peignes ou de filamens attachés au plafond d'une cavité particulière qui s'ouvre en avant, soit sur le dos, soit à gauche, entre le bord du manteau et le corps; deux tentacules; deux yeux diversement situés, quelquefois sur des pédicules; un seul sexe (le sexe femelle), de manière que chaque individu se féconde luimême.

Coq. clypéiforme, recouvrante, très ouverte, à bords continus, quelquefois sans spire; symétrique ou non.

L'ordre des Scutibranches, encore peu étendu, renferme des animaux assez analogues entre eux, mais chez qui l'on remarque cependant quelques différences daus la disposition des branchies. Ils ressemblent assez aux Pectinibran- 
ches; mais ils n'ont point comme ceux-ci les sexes séparés sur des individus différens, et M. Cuvier pense qu'ils n'ont pas besoin d'accouplement pour se reproduire.

Les Scutibranches sont tous marins; on les rencontre particulièrement sur les rochers où ils s'attachent en faisant le vide avec leur pied, et se recourrant de leur coquille; ils rampent peu, et quelques uns d'entre eux semblent même ne jamais quitter le lieu qu'ils ont adopté; on en trouve dans tous les climats, mais en plus grand nombre dans ceux qui sont les plus chauds.

I TE FA MILLE.

LES ORMIERS, Cuv.

Macrostomes, Lam.; Otidés, Blainv.; Auriformes, Lat.

Anim. muni de deux yeux pédiculés à la base extérieure des tentacules; cavité respiratrice située à gauche, et renfermant deux peignes branchiaux inégaux.

Coq. non symétrique, à spire très distincte, munie de canaux particuliers.

\section{G. CXCIII. HaLiotide, haliotis, Lin.}

Anim. oblong, déprimé, muni d'une tête large et d'une trompe courte, à l'extrémité de laquelle est la bouche; celle-ci contenant une langue armée d'aiguillons; deux tentacules longs et cylindriques; yeux sur des pédicules implantés à leur base extérieure, un peu en arrière ; manteau court, mince; pied très large, oblong, muni tout autour d'une double rangée de festons agréablement découpés; organes de la respiration composés de deux peignes branchiaux inégaux, dans une cavité ouverte à gauche, le muscle d'attache occupant le milieu de l'animal; anus s'ouvrant dans cette cavité, vis-à-vis la fente qui en forme l'ouverture.

Coq. auriforme, très déprimée, épaisse, nacrée à 
l'intérieur; à spire très petite, courte, aplatie, postérieure et presque latérale; ouverture très grande, plus longue que large, à bords continus, le droit mince et tranchant, le gauche épais et quelquefois aplati; une série de trous disposés en arc de cercle parallèle au bord gauche, chacun d'eux s'agrandissant en s'éloignant du sommet.

Toutes les espèces de ce genre sont des coquilles agréables par la beauté de leur nacre; elles sont très répandues dans toutes les mers, et nous en possédons une ou deux à l'état fossile.

\section{FAMILIE.}

\section{LES CABOCHONS, Fér.}

Calyptraciens, Lam.; Piléiformes, Lat.

Anim. portant les yeux sur de petits renflemens, à la base extérieure ou un peu au-dessus de la base extérieure des tentacules; organes de la respiration composés de filamens adhérens au plancher de la cavité branchiale.

Coq. piléiforme non symétrique, à sommet parfois spiral.

G. CXCIV. Calyptrée, calyptraea, Lam.

Anim. ovale ou suborbiculaire, plus ou moins déprimé, non spiral; tête bien découverte, large, déprimée, bifurquée en avant avec une bande marginale de chaque côté du cou ; tentacules latétéraux, distans, très grands, triangulaires, fort minces, pointus à l'extrémité, et portant les yeux sur un léger renflement du milieu de leur bord externe ou postérieur; manteau fort mince, sans tentacules latéraux; pied subcirculaire, très peu épais, surtout en avant; cavité branchiale fort grande, oblique de gauche à droite, s'ouvrant largement en avant, et contenant une branchie 
formée de longs filamens roides et exsertiles; anus à l'extrémité d'un petit tube flottant dans la cavité branchiale; un seul muscle d'insertion subcentral. Coq. irrégulière, conoïde; à sommet vertical en pointe et peu postérieur; ouverture très irrégulière, subcirculaire ou oblongue; cavité oblongue, conique, contenant vers le sommet une lame diversement conformée; impression musculaire de forme variable sur cette lame.

Les Calyptrées sont de charmantes petites coquilles généralement fragiles et incolores qui se distinguent parfaitement par la pièce singulière qui est fixée au fond de leur cavité. M. de Lamarck n'en cite que quatre espèces vivantes; mais nous en connaissons un bien plus grand nombre, nous occupant à en faire une monographie, ainsi que des genres voisins. On peut provisoirement établir parmi elles les groupes suivans basés sur la disposition de la lame interne.

I er groupe.

Une lame oblique, adhérente au cône, à progression spirale autour du sommet de la coquille.

C. trochiformis, etc.

$$
2^{\mathrm{e}} \text { groupe. }
$$

Une lame verticale, simple, étroite, adhérente au cône.

C. deformis, etc.

$3^{\mathrm{e}}$ groupe.

Une lame verticale sous le sommet, indépendante du cône et en forme de demi-cornet.

C. plicaria, etc.

\section{$4^{e}$ groupe.}

Une lame verticale sous le sommet, dépendante du cône et en forme de cornet complet.

C. sinense, etc. 
C'est à M. de Blainville, qui, le premier, a observé l'animal, que nous avons emprunté la caractéristique de ce genre dont plusieurs espèces sont fossiles.

\section{G. CXCV. Crépidule, crepidula, Lam.}

Anim. souvent déprimé, ovale, faiblement spiral au sommet; tête bombée, bordée antérieurement par une lèvre bifide, de chaque bifurcation de laquelle part une petite membrane décurrente allant se terminer au point de jonction du corps et du pied; deux tentacules presque cylindriques, gros, obtus, peu contractiles, portant les yeux à leur base extérieure; pied peu épais; manteau mince, sans ornemens latéraux; cavité branchiale très grande, oblique de gauche à droite, s'ouvrant largement, et contenant des branchies en forme de rangée transversale de longs filamens adhérens au plafond, et dont les extrémités peuvent flotter audehors; anus à droite, dans la même cavité.

Coq. irrégulière, ovale ou oblongue, convexe en dessus, et souvent très déprimée, ayant la spire peu formée et fortement inclinée sur le bord postérieur; cavité très grande, à bords irréguliers et tranchans, partagée en deux parties par une cloison horizontale qui prend sa naissance en arrière; impression musculaire en fer à cheval.

Les Crépidules sont des mollusques qui habitent sur les rochers où ils se fixent défnitivement; M. de Lamarck en cite six espèces vivantes; mais il en existe un bien plus grand nombre. Nous en possédons une sur les côtes de Toulon qui se retrouve en grande abondance au cap de Bonne-Espérance, où elle se fixe fréquemment sur d'autres coquilles vivantes, particulièrement une espèce de Pourpse qu'elle suit dans ses mouvemens. 


\section{G. CXGVI. Notrême, notrema, Raf. Trémésie, Raf.}

Anim. mutique, se fixant à la manière des Patelles; tête allongée, tronquée; yeux sessiles.

Coq. composée de trois pièces distinctes et différentes, la première ou intermédiaire grande, ovale, conique, perforée au sommet; la seconde petite, latérale, inférieure, servant de support; la troisième operculiforme et formant le sommet de la première.

M. Rafinesque, qui a établi en si peu de mots ce genre, ne décrit qu'une seule espèce. Il nous paraît tellement extraordinaire que nous serions tenté de dire avec $M$. de Blainville: “ Ne serait-ce pas plutôt une Balanide mal “ observée?"

\section{G. CXCVII. Hipponice, hipponix, Def.}

Anim. ovale ou subcirculaire, conique ou déprimé; tête globuleuse, portée à l'extrémité d'une espèce de cou, de chaque côté duquel est un tentacule renflé à la base, et terminé par une petite pointe conique; yeux sur le renflement tentaculaire; bouche avec deux petits tentacules labiaux; pied fort mince, épaissi vers ses bords, qui s'amincissent et s'élargissent à la manière de ceux du manteau auquel ils ressemblent complétement; branchies situées au-dessus de l'origine du dos; anus au côté droit de la cavité cervicale ; oviducte terminé dans un gros tubercule, à la racine du tentacule droit. Coq. conoïde ou déprimée, à sommet non spiral; ouverture à bords irréguliers ; cavité profonde offrant une impression musculaire en forme de fer à cheval; un support lamelleux, arrondi, ou une empreinte sur le corps servant d'appui, présentant également une impression musculaire en forme de fer à cheval.

Ce genre, établi par M. Defrance pour des coquilles fos- 
siles, n'avait été adopté par M. de Lamarck que comme division du genre Cabochon. M. de Blainville a rétabli le genre Hipponice après l'observation d'un animal rapporté par MM. Quoy et Graimard qui semble lui appartenir, c'est l' $H$ radiata. Ce savant nous fournit par conséquent la caractéristique. Il y a plusieurs espèces fossiles.

\section{G. CXCVIII. Cавоснол, capulus, Montf.}

Anim. conique, légèrement spiral au sommet, muni d'une tête distincte avec une trompe qui termine la bouche; en avant du bord antérieur du pied est une double membrane formant de nombreux replis; les tentacules sont presque cylindriques, gros, obtus, et portent les yeux sur de petits renflemens un peu au-dessus de leur base extérieure; pied grand, très antérieur et mince; manteau simple et sans ornement; cavité branchiale ouverte en avant; branchies composées de beaucoup de lames étroites et longitudinales, qui adhèrent par une seule ligne transversale au plafond; orifice de l'anus vers le côté droit de cette cavité.

Coq. irrégulière, conique, à sommet plus ou moins incliné ou spiral, en arrière; ouverture arrondie, à bords simples, irréguliers et continus; cavité profonde, offrant une impression musculaire en forme de fer à cheval, ouvert en avant.

Ce genre, dont nous possédons une belle espèce sur les côtes de Cette, ne renferme d'après $M$. de Lamarck que quatre espèces vivantes; mais nous pouvons affirmer qu'il en existe bien davantage. Plusieurs d'entre elles sont fossiles.

\section{3e FA MiLLE.}

\section{LES PATELLOIDES, Fér.}

Calyptraciens, Lam.; Branchifères, Blainv.; Piléiformes, Lat.

Anim. muni de deux yeux placés sur de petits renflemens à la base extérieure des tentacules; cavité 
respiratrice ouverte sur le dos, en avant, et contenant deux peignes branchiaux égaux.

Coq. symétrique, munie d'un canal particulier de forme variable.

G. CXCIX. Parmophore, parmophorus, Blainv.

Anim. épais, oblong, déprimé, muni d'une tête peu distincte, avec des tentacules coniques portant les yeux à leur base externe, sur de petits renflemens; manteau dépassant tout le corps, ouvert en avant et offrant en dessus une expansion propre à retenir la coquille, en recouvrant ses bords tout à l'entour; pied très grand, oblong et très épais; cavité branchiale antéro-dorsale, s'ouvrant en arrière de la tête par une fente transversale; branchies formées par deux peignes égaux en forme de pyramide, se réunissant à leur base; anus s'ouvrant au fond de cette cavité, dans la ligne médiane.

L'on doit à M. de Blainville la connaissance de ce geure intéressant, dont nous avons eu occasion d'examiner aussi plusieurs individus envoyés au Jardin du Roi, par MM. Quoy et Gaimard. On en connaît en France deux autres espèces à l'état fossile.

G. CC. Émarginule, emarginula, Lam. Et Rimule, Def.

Anim. ovale, boimbé, portant les yeux sur de gros tubercules, à la base extérieure des tentacules, ceux-ci courts et coniques; manteau très ample, repliant ses côtés sur la coquille, largement ouvert en avant, et muni en outre supérieurement d'une fente qui communique avec celle de la coquille; pied assez large et ovale, muni en dehors et dans sa circonférence d'appendices tentaculiformes; branchies composées de deux peignes égaux; anus 
à l'extrémité d'un petit tube, s'ouvrant dans la cavité respiratrice.

Coq. ovale, conique, plus ou moins élevée, à sommet incliné en arrière; ouverture assez grande, munie d'une échancrure ou d'une scissure à son bord antérieur, ou même sur le dos; empreinte musculaire en fer à cheval.

Le genre Emarginule ne renferme que de petites coquilles bornées à un petit nombre d'espèces. Elles offrent quelques différences dans la disposition de leur entaille, aussi M. de Blainville propose-t-il de les distinguer ainsi :

$$
\text { I }^{\text {er }} \text { groupe. }
$$

L'entaille sur le dos, loin d'atteindre le bord. (Rinule, Def.)

E. blainvilii, etc.

$2^{\text {e }}$ groupe.

Coq. comprimée, une scissure en avant. (Les Entailles.) E. fissura, etc.

\section{$3^{\mathrm{e}}$ groupe.}

Coq. plus comprimée, le bord antérieur plié en gouttière. (Subémarginules.)

$E$. emarginata, etc.

$$
4^{e} \text { groupe. }
$$

Coq. très déprimée; le sommet peu marqué, prémédiant; une petite échancrure en avant.

E. depressa, etc.

Ce genre renferme des espèces dont quelques unes se trouvent sur nos côtes à l'état frais. Il y en a aussi à l'état fossile.

G. CCI. Fissurelle, fissurella, Lam.

Anim. oblong, allongé, bombé, muni d'une tête très distincte et assez large, terminée en avant par une 
trompe courte et arrondie, à l'extrémité de laquelle est la bouche; deux tentacules coniques portant les yeux très saillans à leur base extérieure; manteau grand, mince, ouvert en avant pour la cavité branchiale, et fendu sur le milieu du dos, de manière à établir une nouvelle communication entre le fluide ambiant et cette cavité ; pied très grand et ovaleoblong, muni à l'entour de son bord extérieur de tubercules plus ou moins apparens et diversement conformés; organes de la respiration composés de deux grands peignes branchiaux égaux, se portant très en avant, et naissant de chaque côté de la partie dorsale; anus s'ouvrant à l'extrémité d'un petit tube, en arrière de cette même fente, et toujours dans la cavité respiratrice.

Coq. recouvrante, patelliforme, conique, sans trace de spire, à base oblongue, largement ouverte, avec les bords continus; sommet tronqué et muni d'une ouverture un peu allongée; empreinte musculaire en forme de fer à cheval, ouvert en avant.

Le genre Fissurelle, que l'on a, avec grande raison, séparé des Patelles, puisque leurs animaux sont si différens, est un des plus faciles a caractériser. Ces mollusques unt les mêmes mœurs que les Patelles avec qui ils vivent sur les rochers, ne changent de place que rarement, et comme nous le croyons, pendant la nuit seulement. Il y a plusieurs espèces fossiles.

\section{$9^{\circ}$ ORDRE.}

\section{CIRRHOBRANCHES, Blainv.}

Anim. muni d'un pied antérieur et terminal, allongé en cône pour pénétrer dans le sable; des branchies en forme de longs filamens nombreux, portés par deux lobes radicaux, au-dessus du cou, et enveloppés, ainsi que la tête, par le manteau qui s'ouvre en avant; quelques expansions tentaculaires; yeux? 
un seul sexe (le sexe femelle), l'individu se suffisant à lui-même.

Coq. simple, non enroulée, symétrique, tubuleuse, et en cône très allongé, ouverte aux deux extrémités.

Cet ordre, nouvellement établi par M. de Blainville, ne comprend encore qu'un seul genre qui avait été jusqu'ici rangé parmi les Annélides. M. Deshayes a fait connaître, par un Mémoire anatomique, que les Dentales étaient de véritables mollusques, et nous ne balançons pas un instant à les rapprocher des Fissurelles et des Patelles avec lesquelles nous leur reconnaissons beaucoup d'analogie; en cela nous ne partageons pas complétement l'opinion de $\mathbf{M}$. de Blainville, qui pense devoir aussi les rapprocher des $\mathrm{Nu}-$ cléobranches.

\section{FAMILIE UNIQUE.}

\section{LES DENT ALES.}

\section{G. CCII. Dentale, dentalium, Lin.}

Anim. très allongé, conique, muni d'une tête distincte, petite, ovale; bouche terminale, enveloppée d'appendices labiaux digités, munie d'une paire de mâchoires latérales, ovales et hérissées de petites pointes; yeux? manteau cylindrique enveloppant à peu près la moitié antérieure, terminé en avant par un bourrelet ou collier, au milieu duquel se trouve l'ouverture circulaire du pied; celui-ci toutà-fait antérieur, remplissant presque tout le manteau dans l'état de repos, terminé en avant par une sorte de cupule, du milieu de laquelle s'élève un appendice conique; branchies en forme de longs filamens disposés en deux groupes situés un de chaque côté de la partie supérieure du cou; anus médian à l'extrémité postérieure; organes de la génération inconnus.

Coq. régulière, symétrique, en forme de cône très 
allongé et tronqué au sommet; légèrement courbe, ouverte à chaque ex trémité par un orifice arrondi.

Le genre Dentale, type de l'ordre des Cirrhobranches, renferme un assez bon nombre d'espèces; M. Deshayes a joint à son Mémoire anatomique sur l’animal une monographie dans laquelle il en compte quarante-deux formant quatre sections ou groupes. Nous ne reproduisons que trois de ces groupes, l'un d'eux étant consacré au Dentalium coarctatum de Lamarck qui paraît appartenir à notre sousgenre Créséis du genre Cléodore.

$$
\text { I }{ }^{\mathrm{er}} \text { groupe. }
$$

Coquille non fendue à son extrémité postérieure.

$a$. Des sîries longitudinales.

D. elephantinum, etc.

b. Point de stries longitudinales.

D. entalis, etc.

$2^{e}$ groupe.

Coquille fendue à son extrémité postérieure.

$a$. Des stries longitudinales.

D. striatum, etc.

b. l'oint de stries longitudinales.

D. eburneum, etc.

3 e groupe.

Coquille ayant un bourrelet marginal; non fendue à son extrémité postérieure.

D. strangulatum, etc.

Parmi les espèces de ces différens groupes, il y en a beaucoup de fossiles. 
IO ORDRE.

\section{CYCLOBRANCHES, Cuv.}

Phyllidiens, Lam.; Chismobranches et Polyplaaiphores, Blainv.

Anim. muni d'un pied pour ramper; des branchies pour respirer en forme de feuillets ou de pyramides attachés en cordon plus ou moins complet, sous les rebord̉s du manteau; un seul sexe (le sexe femelle , l'individu se suffisant à lui-même.

Coq. non spirale, recouvrante, d'une ou de plusieurs pièces.

Cet ordre ne renferme encore que deux familles qui comprenuent un genre seulement chaque. Ce sont des animaux marins très répandus dans toutes les mers.

I re FAMiLLe.

LES PATELleS, Fér.

Rétifères, Blainv.

Anim. muni de tentacules et d'yeux à leur base extérieure; des branchies formées par un cordon de feuillets.

Coq. univalve.

\section{G. CCIJI. Patellar, patella, Lin. Et Helcion, Montf.}

Arim. conique, à base circulaire, un peu oblongue, muni d'une tête très distincte terminée par une trompe grosse et courte; deux tentacules pointus portant les yeux à leur base extérieure; bouche charnue renfermant un long ruban lingual épineux qui se porte en arrière, et se replie profondément dans la cavité viscérale; pied grand et arrondi, dépassé dans sa circonférence par les 
bords du manteau; branchies en forme de cordon de petits feuillets branchiaux faisant tout le tour entre le manteau et le pied; anus et orifice de la génération un peu à droite, au-dessus de la tête. Cơ. conique, à base plus ou moins ovale, à sommet droit ou recourbé, toujours dans la ligne médiane; ouverture grande, à bords tranchans et horizontaux; cavité plus ou moins profonde, offrant une empreinte musculaire étroite et en fer à cheval, ouvert en avant.

M. de Blainville croit reconnaître les branchies des Patelles dans un réseau vasculaire qui serait attaché au plafond d'une cavité branchiale; n'ayant point pu distinguer ce réseau, nous pensons avec M. Cuvier que l'organe de la respiration se montre chez ces animaux dans le cordon de féuillets qui entoure tout le corps entre le pied et le manteau, comme on le voit dans les Phyllidies. Les Patelles forment un genre extrêmement nombreux, mais dont les espèces sont souvent très difficiles à distinguer, à cause des variétés singulières que présentent les différens àges; aussi pensons-nous que, pour en former une monographie, il convient de les étudier avec l'animal vivant et en place. M. de Lamarck n'en cite que quarante-cinq comme exemple; mais le nombre en est bien plus grand. Elles sont de toutes les mers, et habitent sur les rochers, où elles se fixent fortement à l'aide de leur pied; ce n'est que rarement qu'elles changent de place, et la nuit seulement, comme nous avons eu lieu de nous en convaincre; elles se trouvent toujours par quantités considérables dans les lieux où la mer brise avec le plus de force. Le cap de BonneEspérance semble être le pays le plus riche en ce genre de coquilles; on y remarque, en effet, un grand nombre d'espèces, toutes fort belles, souvent très grandes, et dont les débris recouvrent les plages en immense quantité. C'est de là que provient la Patelle cuillère qui tire son nom de ce que les Hottentots et les soldats anglais s'en servent 'quelquefuis pour manger la soupe.

M. de Blainville a établi quelques divisions parmi les Patelles, que nous avions déjà en partie faites dans notre collection, et que nous reproduisons à peu près ici. 
I er groupe.

Coq. conique, ovale, à sommet obtus et subcentral.

P. vulgata, etc.

$$
2^{\mathrm{e}} \text { groupe. }
$$

Coq. déprimée, aplatie, à base très anguleuse et comme digitée.

P. ccerulea, etc.

$$
\text { 3e groupe. }
$$

Coq. allongée, comprimée latéralement et souvent obtuse.

P. compressa, etc.

$$
4^{\mathrm{e}} \text { groupe. }
$$

Coq. ovale, à sommet très distinct et recourbé vers le bord antérieur. ( G. Helcion, Montf.)

P. pectinata, etc.

$$
5^{\text {e }} \text { groupe. }
$$

Coq. ovale, mince, quelquefois translucide, à sommet marginal et placé sur le disque même.

P. cymbularia, etc.

$$
\text { 6e groupe. }
$$

Coq. aplatie, à sommet peu marqué, plus étroite en avant qu'en arrière.

$P$. cochlear, etc.

Ce beau genre, sur lequel nous rassemblons depuis longtemps les matériaux propres à une monographie, renferme aussi des espèces fossiles.

$$
2^{\text {e FA MILLE. }}
$$

LES OSCABRIONS, Fér.

Polyplaxiphores, Blainv.; Lamellés, Lat.

Anim. sans tentacules et sans yeux, mais muni d'un petit voile; les branchies formées par un cordon de petites pyramides. 
Coq. multivalve.

\section{G. CCIV. Oscabrion, Lin. Et Oscabrelle, Lam.}

Anim. allongé, demi-cylindrique, obtus aux deux extrémités, et sans tềte bien distincte; tentacules remplacés par un petit voile membraneux qui s'étend sur la bouche; celle-ci antérieure et en dessous, au milieu d'une masse considérable, sans mâchoire, et contenant une petite langue hérissée de denticules; point d'yeux; pied allongé et oblong; manteau le dépassant plus ou moins complétement dans sa circonférence; branchies formées par un cordon de petites pyramides, sous le bord du manteau, particulièrement en arrière; anus sous l'extrémité postérieure; organe de la génération ayant une terminaison double de chaque côté, entre les peignes branchiaux.

Coq. recouvrante, ohlongue allongée, arrondie ̀̀ chaque extrémité, convexe en dessus, concave en dessous, formée de six, sept, et plus souvent huit pièces ou écailles transverses, généralement grandes et imbriquées, et quelquefois petites et simplement rapprochées, ou même distantes, et d'un bourrelet pour les lier ensemble, formé par les bords du manteau; le bourrelet, qui est coriace, se montre lisse ou ridé, chagriné ou écailleux, velu, et même quelquefois épineux.

Ce genre intéressant, et trop peu connu encore, renferme un bon nombre d'espèces, la plupart des pays chauds. M. de Lamarck en a détaché deux pour former son genre Oscabrelle, dont on peut tout au plus faire un sous-genre.

$1^{\text {er }}$ S.-G. Oscabrions proprement dits.

Anim. muni d'un pied oblong, grand, large, quelquefois presque circulaire, le dos en grande partie recouvert. 
MF́THOUIQUE, etc.

Coq. formée de plusieurs pièces imbriquées, grandes, larges, surtout celles du milieu, recouvrant tout le dos de l'animal.

Ce sous-genre renferme le plus grand numbre des espèces. Une ou deux petites seulement sont de nos côtes; mais il en existe de très grandes dans les mers équatoriales.

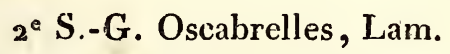

Anim. moins déprimé, muni d'un pied très étroit ; dos en grande partie nu.

Coq. formée de plusieurs pièces rapprochées par leurs extrémités, ou distantes, dans la ligne médiane, petites, surtout celles du milieu; ne recouvrant qu'une très petite partie du dos.

On en connaît au moins trois, qui appartiennent aux mers australes. 


\section{QUATRIEME CLASSE.}

\section{ACÉPHALES, Cuvier.}

Anim. Corps fixe ou libre; point d'organes locomoteurs, ou, comme dans un grand nombre de cas, un pied abdominal, vertical, plus ou moins considérable, ou, comme dans quelques uns seulement, les bords des ouvertures du manteau servant à la natation; point de tête distincte, mais une bouche sans dents, cachée dans le fond ou entre les replis du manteau, souvent munie de chaque côté d'une paire d'appendices; point d'yeux; des organes respiratoires branchiaux, peu variables dans leur forme et leur position; tous se fécondant eux. mêmes.

Coq. toujours extérieure et de deux pièces (dans un petit nombre de cas quelques pièces accessoires); ou nulle, mais alors remplacée par un manteau épais.

Aquatiques, le plus grand nombre étant marins.

Les Acéphales forment une classe non moins nombreuse que celle des Gastéropodes, et qui se distingue facilement des autres, tant par l'organisation de l'animal que par la forme et le nombre des pièces de la coquille. Elle est divisée en quatre ordres : le premier qui répond à la classe des Brachiopodes de M. Cuvier; le second qui comprend les Rudistes de M. de Lamarck; le troisième qui renferme les Acéphales testacés de M. Cuvier, et pour lequel nous empruntons à M. de Blainville la dénomination de Lamellibranches, et le quatrième pour les Acéphales sans coquilles du même, et que nous désignons encore, comme ce savant, sous le nom d'Hétérobranches. 
Tro SECTION. ACÉPHALES TESTACÉS, Cuv.

$$
\text { I }^{\text {er }} \text { ORDRR. }
$$

\section{BRACHIOPODES, Cuv.}

Anim. enveloppé dans un manteau formé de deux lobes toujours ouveris; bouche antérieure, et pourvue d'une paire de bras charnus garnis de filamens, et extensibles au-dehors; les branchies appliquées à la face interne des lobes du manteau; l'anus antérieur. Coq. à deux valves, l'une supérieure, l'autre inférieure, se réunissant en arrière, avec ou sans charnière, et s'ouvrant en avant.

$$
\text { I Te FAMILLE. }
$$

\section{LES LINGULES.}

Anim. allongé, aplati, ayant le manteau ouvert en avant.

Coq. équivalve, équilatérale, régulière, sans charw nière ni ligament, fixée aux corps sous-marins par un pédoncule fibreux.

G. CGV. Lingule, lingula, Brug.

Anim. ovale, allongé, enveloppé d'un manteau ouvert dans toute sa moitié antérieure, et portan des branchies pectinées à sa face interne; bouche munie de chaque côté d'un long bras ou appendice tentaculaire, cilié, se roulant en spirale pour rentrer dans la coquille.

Coq. épidermée, mince, équivalve, équilatérale, allongée, aplatie, tronquée en avant, anguleuse postérieurement pour former le sommet qui est médian, et auquel adhère un long pédoncule fibreux. qui sert à la fixer; impression musculaire composée, centrale et symótrique. 
La $L$. anatina, la seule espèce connue, appartient aux mers australes, et devient assez commune dans les collections. On en connaît de fossiles.

\section{$2^{e}$ FAMILIE.}

\section{LES TÚRÉBRATULES.}

Anim. plus ou moins globuleux ou aplati, ayant le manteau ouvert en avant et sur une partie du côté. Coq. inéquivalve, équilatérale, régulière, s'articulant en arrière au moyen d'une charnière, et se fixant aux corps sous-marins, soit par un pédoncule, soit par l'adhérence de l'une des valves.

A. Coq. se fixant aux corps par le moyen d'un pédoucule.

G. CCVI. Térébratule, terebratula, Brug. Eq Pantastère, Sow.; Strygocépkale, Def.; Spirifère, Magas, Sow.

Anim. plus ou moins globuleux, oblong ou déprimé, ayant le manteau tout ouvert en avant, et en partie sur les côtés, avec les branchies en forme de peigne fixé à sa face interne; deux bras longs et ciliés avoisinant la bouche et se roulant en spirale pour rentrer dans la coquille.

Coq. mince, fragile, inéquivalve, équilatérale, de forme très variable, unie ou striée longitudinalement; l'une des valves plus profonde que l'autre, et prolongée en arrière en un talon recourbé, qui montre toujours une ouverture dans la ligne médiane pour recevoir le pédoncule tendineux qui fixe la coquille aux corps sous-marins; charnière en ligne droite, formée par deux saillies entrant d'une valve dans l'autre; la grande valve offrant en outre dans la cavité un système de charpente, solide et varié selon les espèces, mais toujours formé d'une . partie médiane fortement adhérente en arrière. 
Les Térébratules forment un genre très nombreux dans lequel on ne distingue qu'une douzaine d'espèces à l'état vivant. Elles habitent à de grandes profondeurs, et se fixent sur les corps par l'épanouissement de plusieurs filamens du pédoncule tendineux qui sort de leur ouverture postérieure. Les espèces fossiles sont extrêmement répandues dans toute l'Europe. Une bonne monographie de ce genre est vivement à désirer.

Les Térébratules ont donné lieu à la formation de plusieurs genres nouveaux, mais évidemment artificiels; et si, à l'exemple de M. de Blainville, nous leur rapportons toutes les espèces munies d'une charpente ou support interne, nous pourrons seulement y établir les divisions suivantes qui ont été proposées par ce savant.

$$
\text { xer groupe. }
$$

a. Le talon percé à son extrémité d'un trou rond bien circonscrit.

ז. Valves triangulaires à bord antérieur droit. T. digona, etc.

2. Valves arrondies à leur bord antériear. T. globosa, etc.

3. Valves relevées ou comme échancrées dans la ligụe moyenne.

$T$. sanguinea, etc.

4. Coq. comme bilobée, striée du sommet à la circonférence, et difforme dans la jonction du bord des valves.

T. difformis, etc.

5. Valves comme trilobées par la saillie de la partie moyenne.

T. alata, etc.

$$
2^{\mathrm{e}} \text { groupe. }
$$

b. Le talon de la grande valve profondément échaucré jusqu'au bord de l'articulation.

I. Les valves arrondies à leur bord antérieur.

$$
\text { T. rubra, etc. }
$$


2. Les valves subbilobées par l'échancrure apparente dis bord antérieur.

T. caput serpentis, etc.

$$
3^{\mathrm{e}} \text { groupe. }
$$

c. L'échancrure du talon marginale, triangulaire, et allongée du sommet à l'articulation.

I. Les valves arrondies.

T. lyra, etc.

2. Les valves subbilobées.

$T$. canalifera, etc.

3. Les valves arrondies; une cloison médiane de la grande valve, se plaçant entre deux de la pelite, ce qui, dans le moule en relief, produit cinq pièces distinctes, trois pour une valve et deux pour l'autre. (G. Pantastère, Sowesby.)

$T$. pantastere, etc.

$$
4^{\mathrm{e}} \text { groupe. }
$$

d. L'échancrure du talon marginale, triangulaire, mais bien plus large transversalement que d'avant en arrière; la ligne d'articulation tout-à-fait droite.

I. La petite valve pourvue dans sa partie médiane d'un support droit aplati, bifurqué à son extrémité libre; une cloison de l'autre valve pénétrant dans cette bifurcation. (Gro Strygocéphale, Defr.)

T. burtini, etc.

2. Les parties latérales du support formées par un fila-ment très fin contourné en spirale, de manière à coustituer deux masses creuses coniques qui remplissent presque tonte la coquille. (G. Spirifère, Sowesby.)

$T$. spirifère, etc.

$$
5 \text { e groupe. }
$$

e. La valve supérieure operculiforme ou très plate, le système de support tendant à disparaître.

I, La valve supérieure très plate. (Gr. Magas.)

T. magas. 
2. La valve supérieure très excavée en dessus; le sommet de l'inférieure non percé et divisé en deux parties similaires par un sillon médian bien prononcé. (Productus, Sowe(by.)

$T$. gigantea, etc.

B. Coquille adhérente immédiatement aux corps marins.

\section{G. CCVII. Strophonèm, strophonema, Raf.}

Anim. entièrement inconnu.

Coq. équilatérale, régulière, subéquivalve, ayant une valve plate et l'autre un peu excavée; articulation droite, transverse, offrant à droite et à gauche d'une subéchancrure médiane, un bourrelet peu considérable, crénelé ou denté transversalement; aucun indice de support.

On ne connaît que trois espèces toutes fossiles.

\section{G. CCVIII. Thécrdée, Thecidea, Def.}

Anim, entièrement inconnu.

Coq. équilatérale, régulière, très inéquivalve; valve inférieure creuse, à crochet recourbé, entier, sans échancrure et adhérente, l'autre plate, operculiforme, sans trace de support; charnière longitudinale s'articulant par deux petites dents écartées.

M. Defrance a établi ce genre pour une coquille vivante de la Méditerranée et quatre autres à l'état fossile.

\section{G. CGIX. Galcéole, calceola, Lam.}

Anim. tout-à-fait inconnu.

Coq: épaisse, équilatérale, très inéquivalve, triangulaire, adhérente par la face postérieure de sa valve inférieure; celle-ci très grande, pyramidale, plate en arrière, convexe en avant, à ouverture un peu oblique, demi-circulaire, le bord antérieur étant 
arrondi, et le postérieur droit; celui-ci muni d'une dent au milieu pour former la charnière; valve supérieure operculiforme, aplatie, présentant à son bord postérieur deux petites dents de chaque côté d'une fossette.

Ce genre est formé pour des coquilles fossiles des environs de Juliers. On les a toujours rangées parmi les Rudistes qui sont des coquilles irrégulières, et dont la concamération paraît toute différente. Nous les croyons mieux placées dans le voisinage des Térébratules avec lesquelles elles présentent une grande analogie de caractère. On ne conuaît que deux espèces, les $C$. sandalina et heteroclita; nous en devons une à l'obligeance de M. Hœninghauss de Crefeld, qui est plus grande que les autres, plus aplatie d'avant en arrière, et pourrait peut-être constituer une troisième espèce.

\section{Зе FAMTLLE.}

\section{IES CRANIES.}

Anim. comprimé; le manteau s'ouvrant dans toute sa circonférence.

Coq. inéquivalve, inéquilatérale, irrégulière, sans charnière, se fixant par sa valve inférieure.

\section{G. CCX. Cranie, crania, Brug.}

Anim. pourvu de deux bras ciliés.

Coq. généralement arrondie, inéquivalve, inéquila térale, irrégulière et sans charnière; valve inférieure plus ou moins plane, et présentant quatre impressions musculaires, les deux postérieures écartées, celles du milieu très rapprochées, et se confondant quelquefois; valve supérieure patelliforme, peu élevée, présentant également quatre impressions musculaires.

Ce genre, peu répandu dans les collections, renferme treize espèces, que M. Hœnenghauss vient de faire connaitre 
dans une monographie fort intéressante. Sur ces treize espèces toutes décrites et figurées avec soin, ce naturaliste en compte trois à l'état vivant. Les Cranies se fixent aux corps sous-marins par leur valve inférieure; leur animal ne diffère sans doute pas de celui des Orbicules.

\section{G. CCXI. Orbicure, orbicula, Cuv. Et Discine, Lam.}

Anim. comprimé, arrondi, ayant le manteau ouvert dans toute sa circonférence; bouche avoisinée par deux bras ciliés se roulant en spirale dans la coquille.

Coq. orbiculaire, aplatie, inéquilatérale, inéquivalve, irrégulière; valve inférieure adhérente très mince; la supérieure patellö̈de, recouvrante, à sommet subcentral plus ou moins porté en arrière.

i ${ }^{\text {er }}$ S.-G. Orbicules proprement dites, Guv.

Anim. comme dans la caractéristique du genre. Coq. ayant la valve inférieure non percée.

O. norwegica, etc.

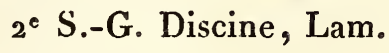

Anim. comme dans la caractéristique du genre. Coq. ayant la valve inférieure percée d'une fente.

$O$. ostreoides, etc.

Les Orbicules sont de nos mers; elles s'attachent aux corps qui ont long-temps séjourné dans certaines profondeurs de la mer, tels que les grosses coquilles, les groupes de Balanes et les IMadrépores; l'on en signale deux espèces fossiles.

$$
2^{\mathrm{e}} \text { ORDRE. }
$$

RUDISTES, Blainv.

Fam. des Rudistes, class. Ch. Des Moul.

Anim. inconnu?

Coq. épaisse, grossière, irrégulière, à texture cello- 
leuse, à valves très inégales; l'inférieure adhérente, presque toujours plus grande que la supérieure, pourvue en général, sur l'une des parois internes, d'une, de deux, ou quelquefois de trois arêtes longitudinales, la supérieure nécessairement libre, souvent beaucoup plus petite, et quelquefois de forme operculaire; contenant un noyau ou birostre d'unè pâte homogène, sans trace d'organisation à l'intérieur; ce noyau est libre, laisse un espace vide entre lui et le test, et présente un appareil accessoire lamelleux, formé de deux lobes, ainsi qu'un bourrelet caréné répondant à la ligne de réunion des deux valves.

L'ordre des Rudistes ne renferme que des coquilles fossiles, dont la connaissance est en partie enveloppée d'obscurité et d'incertitude; aussi les naturalistes ont-ils beaucoup différé dans la manière de les envisager. Nous z’examinerons point quelles ont été les opinions de chacun d'eux; nous donnerons seulement un coup d'œil rapide sur celles de ces opinions qui sont les plus récentes, et qui, en même temps, ont répanda le plus de lumières sur ces curieuses dépouilles de mollusques antédiluviens.

Il est à remarquer qu'en général les auteurs qui on't fait des classifications systématiques, n'ont pas cru devoir éloigner les Rudistes des Huîtres ou des genres qui les avoisiment. En effet, M. Cuvier comprend les Acardes dans la famille même des Huîtres; $M$. de Lamarck en forme une famille qu'il place immédiatement après celle des Ostracés, et avant celle des Brachiopodes; M. de Férussac adopte l'idée de M. de Lamarck, puisqu'il les met entre les Cranies et les Huîtres; M. de Blainville fait de même pour la place qu'il leur assigne; mais il les élève au rang d'ordre. Enfin M. Latreille ne s'écarte que très peu des indications de ses prédécesseurs. Il résulte de là que c'est avec les Huitres d'une part et les Orbicules, Cranies, etc., de l'autre, que l'on a trouvé que les Rudistes avaient le plus d'analogie; mais ces savans naturalistes, en s'accordant ainsi, s'étaient-ils bien fondés dans leur opinion sur des caractères importans? C'est ce que nous ne croyons pas, 
car eux-mêmes connaissaient alors si peu les Sphérulites, qui forment le genre principal des Rudistes, qu'à l'exception de M. Cuvier qui ue dit que peu de chose sur les Acardes, ils faisaient un genre distinct, sous le nom de Birostrite, du noyau qu'elles contiennent. Il faut donc croire qu'ils n'ont été conduits à ce rapprochement, que nous sommes d'ailleurs porté à approuver aujourd'hui, que par une certaine analogie vaguement indiquée dans les formes extérieures de ces coquilles. Mais voici les opinions de deux naturalistes, MM. Charles Des Moulins et Deshayes, qui, tout récemment, ont traité des Rudistes sous un nouveau point de vue. On doit au premier un travail fort important à ce sujet, et d'autant plus précieux, qu'avant de le faire il avait consacré plusieurs années à la réunion d'un nombre considérable de ces fossiles, de manière à former une collection unique en ce genre. C'est en étudiant ses nombreux échantillons, et en les comparant attentivement entre eux, que M. Des Moulins est parvenu à s'en faire une idée juste, et à nous dévoiler un grand nombre de caractères qui n'avaient point encore été a perçus.

M. Des Moulins voit dans les Sphérulites les dépouilles testacées de mollusques fort éloignés des Lamellibranches et des Brachiopodes. Ces coquilles étant toujours composées de deux parties distinctes, le test extérieur et un noyau qu'il nomme birostre, tous deux séparés par un espace vide, il pense que le mollusque qui habitait les Sphérulites, était pourvu d'un manteau dur et épais, ce qui expliquerait assez bien la formation du birostre et du vide, car le premier serait le noyau calcaire formé à la place de l'animal dans l'intérieur de son manteau, et le second résulterait de la disparition plus tardive de celui-ci. Partant de là, il suppose quelques caractères qui auraient appartenu à l'animal, il en indique les traces naturellement empreintes sur le birostre, et, ne connaissant que les Ascidiens qui soient munis d'un manteau semblable, il croit que les Sphérulites doivent, sous ce rapport, se rapprocher des Hétérobranches. (T'uniciers, Lam.) Examinant ensuite la texture des Sphérulites, il y reconnaît les traces d'une cellulosité qu'il compare à celle des Balanides, et il en tire la conséquence que peut-être elles doivent se ranger non loin 
des Cirripèdes; il voudrait donc les voir entre les Hétẻrobranches et les Cirripèdes, car la présence de leur test et ses caractères s'opposent à leur introduction dans l'un ou l'autre de ces groupes d'animanx. Enfin, par suite de ces deux rapprochemens, il conclut à l'établissement d'une classe intermédiaire sous le nom de Rudiste.

M. Deshayes, qui ne paraît pas avoir fait, comme M. Des Moulins, une longue étude des Sphérulites sur une suite considérable d'échantillons précieux, n'a fait que publier à leur sujet l'idée qu'il s'est formée du vide que l'on remarque entre le birostre et le test, et dont la cause, ingénieusement expliquée, le conduit à un résultat tout-à-fait opposé à celui de M. Des Moulins, puisqu'il tendrait à ramener les Sphérulites, etc., à l'état normal des coquilles de Lamellibranches.

Ayant remarqué que certaines coquilles, les Cames, par exemple, sont composées de deux couches distinctes, l'une intérieure et l'autre extérieure, il pense que les Sphérulites étaient dans le même cas; alors il voit dans le birostre le moule parfait de la cavité intérieure des valves réunies, et dans le vide qui entoure ce birostre celui qui serait résulté bientôt après de la dissolution de la couche intérieure du test par le séjour dans la craie. Le birostre donne, par conséquent, selon lui, les caractères parfaits de l'intérieur de chaque valve, tels que des dents, le ligament et les impressions musculaires, et dès-lors les Rudistes rentrent parmi les Lamellibranches et dans le voisinage des Cames sans distinction de familles.

Nous n'arons pas la prétention de combattre les opinions de ces deux naturalistes plus instruits que nous, sans doute, sur un sujet qu'ils ont étudié et mûri longuement; encore bien moins celle d'émettre un nouveau système sur cette matière intéressante; mais nous ferons quelques observations dans le but d'appuyer l'opinion de M. de Blainville, dont nous adoptons complétement la manière d'envisager les Rudistes sous le rapport de la place et du rang qu'ils doivent occuper dans la série des Acéphales.

L'étude des animaux mollusques fait connaître un fait incontestable, c'est que leur manteau, cette enveloppe charnue qui recouvre leur corps, est d'autant plus épais et 
dur, que leur enveloppe testacée est moins solide et propre à les abriter, et vice versâ. Ainsi les $\Lambda$ scidies, etc., sont revêtues d'un manteau épais et coriace; c'est qu'elles n'ont point de coquille du tout. Les animaux des Bulles, Yets, Harpes, Glycymères, Pholades, etc., sont presque dans le même cas; c'est que ces animaux ne reçoivent pas un abri complet de leur coquille. Enfin les Huîtres, Spondyles, Peignes, etc., ont, au contraire, le manteau mince et ouvert; c'est parce que leurs coquilles sont fort épaisses, solides et parfaitement closes. La raison de ces anomalies dans l'organisation extérieure des mollusques, est facile à saisir; on concoit qu'babitant les rivages au milieu des corps sans cesse agités par le mouvement de la mer, ils ont tous besoin d'une enveloppe protectrice; dans un grand nombre de cas, cette enveloppe est ce que nous nommons la coquille, et dans d'autres, ce n'est que le manteau lui-même; mais nous ne voyons pas, et, en effet, cela n'était pas nécessaire, que la nature ait appliqué ces deux moyens à la fois sur le même animal. Nous concluons donc, par le fait de l'expérience, comme par celui du raisonnement, que l'opinion de M. Des Moulins, qui donne aux Rudistes, outre leur test, si compacte et si solide, un manteau épais et coriace, n'est pas admissible. La nature ne fait rien d'inutile, se plaît-on à dire, et tout porte à croire qu'un manteau, ainsi conformé, l'eût été tout-à-fait. Cette idée adoptée, les caractères supposés à l'animal lui-même, et reproduits par le birostre, comme moule interne du manteau, disparaissent, et le rapprochement artificiel, établi entre les Rudistes et les Hétérobranches, n'existe plus. M. Des Moulins pense, en outre, que les Rudistes doivent être rapprochés des Cirripèdes, parce qu'après avoir étudié la cellulosité du test de ces coquilles, il la croit analogue à celle du test des Balanides. Nous ne partageons point encore cette opinion, ces deux sortes de cellulosités nous paraissant toute différente; l'une, celle des Spérulites, semble inhérente au tissu de la coquille; tandis que l'autre nous paraît indépendante de celui des Balanides : dans le premier cas, elle ne se manifeste que dans le tissu même du test sans aucun rapport intime avec la cavité de la coquille non plus qu'avec l'animal, et dans le second, elle se présente sous l'aspect de carités particulières ouvertes à la base et à l'intérieur de la co- 
quille, où elles se montrent plus larges qu'à leur sommet, pour receroir, comme M. Cuvier l'a démontré, de petites expansions du manteau du mollusque.

Nous ne saurions adopter non plus le rapprochement des Rudistes avec les Balanides fondé sur une certaine analogie entre la valve supérieure des premiers et l'opercule des secondes. Dans les uns, c'est une véritable valve d'une seule pièce, peut-être quelquefois de forme operculaire, et toujours externe, et dans les autres, c'est réellement un opercule formé de plusieurs pièces et constamment interne, par rapport à la cavité de la coquille.

M. Deshayes nous semble avoir mieux expliqué la manière dont s'opère le vide entre la coquille des Sphérulites et son noyau ou birostre, en supposant que la couche interne de cette coquille a été dissoute par la craie. On a effectivement des exemples de cette décomposition dans beaucoup de corps fossiles, et l'examen de la paroi interne d'une Sphérulite bien conservée entraîne vers cette idée. Ainsi donc le birostre serait le moule parfait de la cavité de la coquille, lorsqu'elle était intacte. Nous admettrons bien, avec ce naturaliste, sur ce birostre, la présence d'impressions musculaires, déjà signalées par M. Des Moulins; mais nous ajouterons que nous avons vainement cherché sur plusieurs échantillons bien conservés, quelques indices des grosses dents que M. Deshayes signale, et qui établissent selon lui le rapprochement des Rudistes avec les Cames. Nous nous sommes servi pour obtenir une image parfaite de la cavité des Sphérulites du moyen qu'il indique lui-même; c'est-à-dire que nous avons pris avec du mastic le moule du birostre, et si nous n'avons pas vu, après cette opération, des dents, comme il les annonce, nous avons vu les vides laissés par l'appareil accessoire qu'a décrit M. Des Moulins, et qui vient peut-êtro s'opposer au rapprochement proposé par M. Deshayes. Qu'est-ce donc que cet appareil accessoire? et à quoi répondrait-il dans les Cames? Si c'est au ligament, cela nous paraît difficile à démontrer. Un autre embarras dans lequel ce naturaliste doit encore se trouver, c'est celui d'expliquer toujours dans cette idée de rapprochement des liudistes avec les Cames, l'emploi de ces deux ouvertures si constantes dans la valve supérieure des Hippurites 
et de ces deux arêtes qui parcourent dans toute sa longueur la paroi interne de leur valve inférieure. Ne doiton pas voir aussi dans la cellulosité des Rudistes, si bien décrite par M. Des Moulins, un caractère important propre à écarter ces dépouilles testacées de l'ordre des Lamellibranches. Enfin voici une dernière observation: dans toutes coquilles provenant de mollusques Lamellibranches, le point d'origine de chaque valve est toujours plus ou moins latéral, et les lignes d'accroissement vont en s'étendant d'un còté, mais point de l'autre, parce qu'elles ne sont pas précisément concentriques. Ce fait existe pour les Cames comme pour les autres, et dans les Rudistes c'est tout le contraire; le centre de chaque valve en est aussi le centre d'origine, et ces lignes vont en s'étendant concentriquement avec l'âge ; ce caractère n'est certainement pas le moins important.

Quoi qu'il en soit, ces naturalistes ont jeté de grandes lumières sur cette partie de la science; M. Des Moulins en faisant connaître les Sphérulites, telles qu'elles nous ont été laissées, c'est-à-dire en nous montrant que la Birostrite en est le noyau, car c'est à lui le premier que l'on doit cette découverte importante; en ramenant à leur véritable place les Radiolites et les Jodamies, dont on avait à tort fait des genres distincts; en décrivant l'appareil accessoire et plusieurs autres caractères importans qui n'avaient point été remarqués; en fournissant une foule de détails intéressans sur ces coquilles, et enfin en distinguant un bon nombre d'espèces qui n'étaient point connues jusques-là ou bien qui avaient été confondues ensemble; M. Deshayes en expliquant comment s'est opéré le vide entre le birostre et les valves qui le contiennent, et dévoilant par là les caractères probables de l'intérieur de ces valves. Ce naturaliste poussera, sans doute, plus loin ses recherches, comme le fait aussi de son côté M. Des Moulins; et mieux connues, ces curieuses coquilles viendront définitivement prendre la place qui leur convient dans la série des mollusques. En attendant, nous allons nous guider pour la connaissance des genres par le travail de ce dernier savant. 
FAMILLE UNIQUE,

LES ÁCARDES, Charles Des Moulins.

Ruclistes, Lam.

G. CCXII. Śphérulite, spharulites, de Lamétherie. Et Birostrite, Radiolite, Lam.; Jodamie, Def.

Anim. inconnu.

Coq. presque toujours très inéquivalve, généralement pourvue à l'extérieur de lames, écailles ou rides horizontales; queiquefois plissée longitudinalement, formant un cône quelquefois double; valve inférieure adhérente, soit par un de ses côtés, quand elle forme un cylindre ou un cône renversé, soit par son sommet, dans le cas contraire, présentant quelquefois une ou deux arêtes à l'intérieur, ou n'en montrant aucune; valve supérieure plus ou moins conique ou aplatie; birostre formé de deux cônes plus ou moins pointus, légèrement arqués en dedans, se dilatant quelquefois dans le sens horizontal; appareil accessoire presque aussi grand que les cônes.

Le genre Sphérulite, tel que le présente aujourd'hui M. Ch. Des Moulins, comprend, outre les Sphérulites de Lamarck, ses Radiolites et Birostrites, plus les Jodamies de M. Defıance. Nous avons déjà dit que ce naturaliste avait reconnu que la Birostrite n'était autre chose que le moule intérieur des Sphérulites, ainsi ce genre est annulé. Quant aux Jodamies et aux Radiolites, il prouve, par des comparaisons faites avec beaucoup de soin, que ce ne sont que des Sphérulites : on conçoit qu'il fallait être entouré, comme lui, d'un nombre considérable de ces coquilles qui ne se montrent jamais complétement entières pour reconnaître l'abus de ces doubles emplois.

M. Des Moulins, ayant décrit un certain nombre d'espèces nouvelles de ce genre, forme des groupes comme is suit : 
I ${ }^{*}$ groupe. Cratériformes.

Coq. formant un cône droit, court, à base fort élargie et adhérente; tous beaucoup plus épais à la base de la coquille qu'à l'orifice de la valve inférieure; valve supérieure reposant horizontalement sur l'ouverture; birostre à cônes subégaux, courts, épais; appareil accessoire anomal, et du reste peu connu.

$S$. crateriformis, etc.

$2^{\mathrm{e}}$ groupe. Cylindroïdes.

Coq. à base non élargie, hérissée de toute part d'écailles horizontales; épaisseur du test à peu près égale dans toute la lougueur de la grande valve; valve supérieure reposant horizontalement sur l'ouverture; cônes du birostre très inégaux.

S. cylindracea, etc.

\section{3e groupe. Duploconoïdes. (Radiolites, Lam.)}

Coq. dont les valves forment deux cônes opposés par leur base, ou deux cônes opposés à base commune; valve supérieure reposant horizontalement sur l'ouverture; extérieur strié longitudinalement; intérieur peu connu.

$S$. rotularis, etc.

\section{$4^{\mathrm{e}}$ groupe. Cunéiformes.}

Coq. un peu déprimée, et courbée du côté postérieur; écailles extérieures généralement petites, quelquefois presque nulles, non inclinées; valve supérieure très déprimée; ouverture suboblique; cônes $d u$ birostre très inégaux : valve inférieure adhérente par le còté postérieur.

S. cristata, etc.

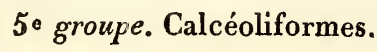

Coq. dont la valve inférieure, turbinée, est ou tout-àfait plate, ou très peu bombée d'un côté, ce côté étant couvert de très grandes écailles, dirigées de la base de la coquille vers l'ouverture, appliquées, 
imbriquées, recouvrantes, très lisses; ouverture transversalement ovale, très oblique; valve supérieure très déprimée, souvent plate; cônes du birostre très inégaux.

$S$. ingens, etc.

G. CCXIII. Hippupite, hippurites, Lam. Et Batolite, Raphaniste, Montf.; Amplexus, Sow.; Orthocéritites, Pic de la Peyr.

Anim. inconnu.

Coq. excessivement inéquivalve, jamais hérissée de lames ou écailles horizontales, cylindracée, plus ou moins allongée; valve inférieure cylindrique ou conique, atténuée, droite, ou plus ou moins recourbée, adhérente par le côté, à arêtes obtuses, parallèles et convergentes, au nombre de deux ou trois; quelquefois, à la place des arètes, lorsqu'elles manquent, ou bien avec ses arêtes, lorsqu'elles existent, une sorte de faux siphon cloisonné comme la coquille; valve supérieure plate, ou quelquefois un peu bombée, operculiforme, dont les bords sont taillés en biseau; birostre à deux lobes très obtus; appareil accessoire incomplétement connu.

Ce genre, beaucoup moins connu que le précédent, renferme un grand nombre d'espèces parmi lesquelles $M$. Des Moulins n'a point établi de coupes, par la raison que la plupart ue lui sont connues que par les figures que l'on en a publiées, et qui ne sauraient être suffisamment caractérisées.

\section{3e ORINRE.}

\section{LAMELLIBRANCHES, Blainv.}

Anim. enveloppé d'un manteau à deux lobes, variant: dans le nombre et la dimension de ses ouvertures; bouche transverse, médiane, cachée dans le fond du manteau, entre deux paires d'appendices; branchies en forme de lames semi-circulaires, an nombre 
de deux paires, une de chaque côté du corps; anus postérieur ét également médian.

Coq. composée de deux valves latérales, s'articulant supérieurement par une charnière et un ligament, et s'ouvrant inférieurement, contenant tout l'animal, auquel elle adhère par des muscles adducteurs.

\section{I $^{\text {re }}$ DIVISION. LES MONOMYAIRES.}

Une seule impression musculaire à chaque valve.

I re FAMILIE.

\section{LES OSTRACÉS, Cuv.}

Anim. muni d'un manteau non adhérent, entièrement ouvert, excepté à la partie dorsale, sans tube ni ouverture particulière; pied nu ou rudimentaire; les deux paires de branchies réunies dans la ligne médiane.

Coq. inéquivalve, inéquilatérale, irrégulière, plus ou moins lamelleuse; charnière variable; impression musculaire subcentrale.

\section{G. CCXIV. Anomre, anomia, Brug.}

Anim. très comprimé, ayant les bords du manteau minces et garnis à l'extérieur d'une rangée de filamens tentaculaires; pied rudimentaire; muscle adducteur divisé en trois branches, dont la plus grande passe par une échancrure de la valve inférieure pour s'attacher à une pièce operculaire, pierreuse ou cornée, fixée aux corps marins.

Coq. adhérente par sa pièce operculaire, inéquivalve, inéquilatérale, irrégulière, assez mince et souvent translucide; valve fixe, plus aplatie, ayant une échancrure ronde ou oblongue près de son crochet pour recevoir la pièce operculaire, l'autre plus grande et plus concave; toutes deux liées par un 
ligament court et épais; impression musculaire divisée en trois.

Ce genre, qui renferme plusieurs espèces de nos côtes, est très facile à reconnaitre, au moyen de la pièce operculaire qui sert à fixer la coquille; cependant $M$. de Blaiuville cite une espèce, l' $A$. squamata, qui n'aurait point cette pièce, et se fixerait par sa valve même. Ce savant observe que les Anomies sont très difficiles à distinguer entre elles; et en effet, nous nous sommes fréquemment aperçu, sur les rivages de la Méditerranée, où ces coquilles sont extrêmement communes, qu'uu individu d'une espèce parfaitement lisse se montrait strié à la manière des Peignes, sur les deux valves mêmes, lorsqu'il s'était fixé sur l'une de ces coquilles. On en connaît plusieurs à l'état fossile.

\section{G. CCXV. Placune, placuna, Brug.}

Anim. extrêmement comprimé, du reste inconnu. Coq. libre, irrégulière, très aplatie, à valves minces, translucides, presque égales et subéquilatérales; charnière interne, offrant sur une valve deux côtes longitudinales, tranchantes, convergentes au sommet, et sur l'autre deux sillons correspondant à ces côtes, et donnant attache au ligament; impression musculaire subcentrale, assez petite.

Ce genre, parfaitement caractérisé par sa charnière, ne renferme que trois espèces, toutes des mers de l'Inde. On le connaît aussi à l'état fossile.

\section{G. CCXVI. Hakpace, harpax, Bronn.}

Anim. inconnu.

Coq. adhérente, irrégulière, subtriangulaire, oblongue, inéquivalve, inéquilatérale; une valve plate et l'autre concave; charnière formée par deux dents longues, crénelées, divergentes du sommet, sur une valve, et se plaçant entre deux paires de dents de même forme, de l'autre; impression musculaire et ligament inconnus. 
Nous ne connaissons point le genre Harpace, établi pour une espèce fossile; mais nous le décrivons d'après $M$. de Blainville, et le plaçons de la même manière que ce savant, à la suite des Placunes, en observant toutefois qu'il élève des doutes sur ce dernier point, pensant que peut-être cette coquille serait mieux auprès des Trigonies.

\section{G. CCXVII. Huître, ostrea, Lam.}

Anim. très comprimé, plus ou moins oblong ou orbiculaire, ayant les bords du manteau épais, non adhérens, rétractiles, ornés de petits appendices tentaculaires courts et irrégulièrement disposés; bouche large, formant l'entonnoir, avoisinée par deux paires d'appendices labiaux, triangulaires et branchiaux ; branchies formées par quatre feuillets presque égaux et semi-circulaires, finement striés en travers; anus postérieur, ayant son orifice sessile. Coq. adhérente, inéquivalve, inéquilatérale, très irrégulière, épaisse, plus ou moins grossièrement feuilletée; à crochets inégaux; valve gauche généralement plus grande et plus concave que la valve droite, son sommet formant souvent avec l'âge une sorte de talon; valve droite plus petite, généralement aplatie, quelquefois operculiforme ; charnière sans dents; ligament presque interne, s'insérant dans une fossette cardinale croissant avec le sommet; impression musculaire subcentrale.

Ce genre, très nombreux en espèces vivantes ou fossiles, est de toutes les mers. On peut les diviser en deux groupes, comme le propose M. de Lamarck.

$$
\text { I er groupe. }
$$

Coquilles non plissées.

o. edulis, etc.

$$
2^{\text {e }} \text { groupe. }
$$

Espèces plissées.

o. crista galli, etc. 


\section{G. CCXVIII. Gryphíe, gryphasa, Lam.}

Anim. inconnu.

Coq. inéquivalve, inéquilatérale, à sommets saillans recourbés en spirale, plus ou moins inclinés sur la gauche; valve gauche généralement très grande et concave; valve droite plus petite et souvent operculiforme; charnière sans dents; ligament s'insérant dans une fossette allongée; impression musculaire subcentrale.

ILe genre Gryphée ne renferme qu'une seule espèce vivante, la $G$. angulata, qui est d'une extrême rareté; mais aussi il en comprend un grand nombre à l'état fossile. Nous pensons que l'on pourrait établir une division en séparant les espèces dont la valve droite ou supérieure est operculiforme, de celles où cette même valve, presque aussi grande et concave que celle de gauche, présente un sommet également en spirale.

\section{$2^{\text {e FAMILLE. }}$}

\section{LES PECTINIDES, Lam.}

Ostracés, Cuv., Fér.; Subostracés, Blainv.; Ostracés et Pectinides, Lat.

Anim. muni d'un manteau non adhérent, presque entièrement ouvert dans toute sa circonférence, sans tube ni ouverture particulière, ayant toujours à la partie abdominale un rudiment de pied, souvent. canaliculé, qui sépare les deux paires de branchies. Coq. en général subrégulière, équivalve ou inéquivalve, à test compacte, non feuilleté dans son épaisseur, à côtes ou stries divergentes des sommets; souvent auriculée; à charnière variable fixée soit par un byssus, soit au moyen de l'une des valves. 


\section{G. CCXIX. Ponopside, podopsis, Lam.}

Anim. inconnu.

Coq. inéquivalve, subrégulière, subéquilatérale, adhérente par l'extrémité de sa valve la plus courte; l'autre ayant le sommet allongé, pointu, un peu recourbé; charnière sans dents, mais offrant deux petites éminences très écartées; ligament intérieur; impression musculaire?

Ce genre ne renferme qu'un très petit nombre d'espèces, toutes fossiles.

G. CGXX. Spondyle, spondylus, Lin.

Anim. épais, ayant le manteau presque entièrement ouvert; appendices labiaux épais et frangés ; pied très petit et sans byssus.

Coq. solide, épaisse, hérissée ou rude, adhérente par la valve droite; inéquivalve, inéquilatérale, assez régulière, subauriculée; valve droite un peu plus grande que la gauche, plus concave, présentant à son sommet une sorte de talon triangulaire, aplati, divisé par un sillon s'agrandissant avec l'âge; charnière longitudinale, composée sur chaque valve de deux fortes dents intrantes, correspondant à deux larges fossettes; ligament court, presque médian, subintérieur, s'enfonçant dans le sillon du talon; impression musculaire supérieure.

Ce genre, l'un des plus beaux par l'éclat des coquilles qu'il renferme, et par la variété de leurs ornemens, comprend un grand nombre d'espèces à l'état vivant, la plus grande partie des mers de l'Inde et de celle des Antilles. Leurs animaux vivent à d'assez grandes profondeurs, se fixant sur les corps sous-marins, et, comme nous avons eu lieu de le remarquer quelquefois, sur les ancres, canons, et autres objets en fer abandonnés. On en connaît aussi plusieurs espèces à l'état fossile, 


\section{G. CCXXI. Hinnite, hinnites, Def.}

Anim. inconnu.

Coq. épaisse, presque régulière, inéquivalve!, subéquilatérale, auriculée, ayant la valve droite très concave, avec une sorte de talon, et la valve gauche aplatie; charnière sans dents; ligament s'insérant dans une fossette en partie extérieure; impression musculaire?

Ce genre, connu seulement à l'état fossile, ne contient que deux ou trois espèces.

\section{G. CCXXII. Pligatule, plicatula, Lam.}

Anim. inconnu.

Coq. solide, épaisse, rude, adhérente, irrégulière, subéquivalve, inéquilatérale, sans talon, anguleuse au sommet, arrondie et ondulée inférieurement; charnière offrant deux fortes dents striées sur chaque valve; une fossette médiane pour l'insertion du ligament, qui est tout-à-fait intérieur; impression musculaire subcentrale.

Les Plicatules, qu'il est toujours facile de distinguer des Spondyles, ne renferment que cinq espèces vivantes; mais il y en a un plns grand nombre à l'état fossile. Toutes les premières sont exotiques.

\section{G. CCXXIII. Peigne, pecten, Brug.}

Anim. orbiculaire, souvent épais, et quelquefois très aplati; manteau bordé par une ou deux rangées de filets très fins, parmi lesquels on remarque souvent, de distance en distance, de petits globules perlés; pied petit, conique, canaliculé, et portant généralement un byssus; bouche entourée d'appendices tentaculaires, branchus et irréguliers, remplaçant les feuillets labiaux ordinaires; bran- 
chies assez grandes; terminaison du canal intestinal un peu en dessous.

Coq. souvent très mince, à tissu serré; libre, régulière, inéquivalve, équilatérale, auriculée, presque toujours rayonnée, à bord supérieur droit; sommets contigus; charnière sans dents; une fossette triangulaire, intérieure, pour recevoir le ligament, et en outre une membrane ligamenteuse occupant toute la longueur de la charnière; impression musculaire subcentrale.

Ce genre, l'un des plus beaux de la classe des Acéphales, renferme un grand nombre d'espèces vivantes, et, d'après M. Defrance, un plus grand encore à l'état fossile. Les Peignes sont de toutes les mers, et se font remarquer généralement par l'éclat de leurs couleurs. $M$. de Blainville paraît porté à croire que lorsque la valve gauche est plus bombée que la valve droite, l'animal est muni d'un byssus, et qu'au contraire il en est privé lorsque c'est la droite qui est plus bombée que la gauche. Les Peignes habitent à d'assez grandes profondeurs. Ceux qui sont entièrement libres se meuvent sur les fonds sablonneux en frappant l'une contre l'autre leurs deux valves.

\section{G. CCXXIV. Dranchore, dianchora, Sow.}

Anim. entièrement inconnu.

Coq. mince, adhérente, régulière, symétrique, équilatérale, subauriculée, inéquivalve; une valve creuse en dedans, bombée en dehors, l'autre plate; articulation par deux condyles bien distans.

Ce genre est établi pour trois espèces fossiles.

\section{G. CGXXV. Houlette, pedum, Brug.}

Anim. inconnu, portant un byssus.

Coq. assez mince, à tissu serré ; subtriangulaire, subauriculée, inéquivalve, inéquilatérale, baillante par une échancrure de sa valve droite seulement; 
sommets obtus, inégaux, écartés; valve droite plus grande que la gauche, bombée, élargie, et un peu recourbée de chaque côté vers la valve gauche; celle-ci plus petite, plus aplatie et sans échancrure; charnière sans dents, oblique; ligament en partie extérieur, inséré dans une fossette allongée et canaliforme de la face interne des sommets, prolongée en dedans sur une sorte de cuilleron.

Ce genre, encore assez rare dans les collections, renferme deux espèces fort voisines, appartenant aux îles de la mer des Indes; on ne les trouve qu'à d'assez grandes profondeurs, fixées par leur byssus sous les rochers.

\section{G. CCXXV1. Plagiostome, plagiostoma, Sow.}

Anim. inconnu.

Coq. assez épaisse, régulière, libre, subéquivalve, subauriculée; bord supérieur droit et transvere; sommets un peu écartés, leur surface interne offrant une facette triangulaire, aplatie et externe, l'une droite, l'autre oblique; charnière sans dents, mais offrant deux condyles latéraux et distans; une fossette conique s'ouvrant en dehors pour l'insertion du ligament.

Ce genre devra probablement être réuni aux Limes; il ne renferme que des espèces fossiles.

\section{G. CCXX VII. LIme, lima, Brug.}

Anim. plus ou moins épais, oblong, ayant les bords de son manteau garnis de plusieurs rangées de filets tentaculaires; pied très petit, et portant un byssus; bouche entourée d'un appendice labial fort épais et frangé.

Coq. ovale, plus ou moins épaisse, subéquivalve, quelquefois presque équilatérale, baillante en avant pour le passage du byssus; sommets écartés; bord 
supérieur droit et longitudinal; charnière sans dents; ligament à moitié extérieur, arrondi, s'insérant dans une fossette assez large de chaque valve; impression musculaire centrale et trifide.

Le genre Lime contient peu d'espèces vivantes, et quelques unes d'elles sont rares; il y en a aussi de fossiles.

\section{3e FAMILLE.}

\section{LES MALLÉACÉS, Lam.}

Ostracés, Cuv.; Oxigones, Lat.; Margaritacés, Blainv.

Anim. ayant le manteau non adhérent, entièrement ouvert dans toute sa circonférence, sans tube ni ouverture particulière, se prolongeant en lobes assez irréguliers, surtout en arrière ; un pied canaliculé, offrant presque toujours un byssus.

Coq. noire ou cornée, inéquivalve, inéquilatérale, très irrégulière; charnière sans dents; le ligament marginal, sublinéaire, simple ou interrompu par des crénelures; l'impression musculaire subcentrale; se fixant généralement au moyen d'un byssus fourni par l'animal.

\section{G. CCXXVIII. Posmonie, posidonia, Bronu.}

Anim. inconnu.

Coq. très mince, presque membraneuse, équivalve, inéquilatérale, oblique, arrondie, non baillante? bord cardinal droit, un peu prolongé de part et d'autre en auricule; charnière édentule; point de fossette pour les ligamens, à l'intérieur comme à l'extérieur, non plus que de sinus pour le passage d'un byssus.

Ce genre a été établi récemment pour des empreintes assez communes sur des schistes provenant de Dillemburg, de la Clappe, etc., et que quelques naturalistes avaient été tentés de rapporter aux tests rudimentaires des Aplysies 
on des Pleurobranches. M. Bronn a reconnu qu'elles provenaient, au contraire, de coquilles bivalves, parce qu'il a $\nabla u$ dans ces empreintes inéquilatérales, le sommet tantôt d'un côté et tantôt de l'autre, les valves étant toujours considérées du même côté, c'est-à-dire en dedans ou en dehors. Cette observation, que nous avons pu facilement vérifier, est concluante, et d'après cela la Posidonie paraît devoir prendre sa place à côté des Limes, non loin des Malléacés.

\section{G. CCXXIX. Vulselee, vulsella, Lam.}

Anim. allongé, comprimé; le manteau très prolongé en arrière, et bordé de deux rangs de tubercules papillaires très serrés; pied petit, canaliculé, sans byssus; bouche grande; appendices labiaux très développés et triangulaires; branchies étroites, très longues, et réunies dans presque toute leur étendue.

Coq. subcornée, mince, allongée, aplatie, irrégulière, inéquilatérale, subéquivalve, à sommets presque antérieurs, distans, et un peu recourbés; charnière sans dents, et offrant simplement sur chaque valve une callosité saillante comprenant une fossette pour l'insertion du ligament; impression musculaire subcentrale.

Ce genre ne renferme qu'un petit nombre d'espèces toutes exotiques. Il y en a une fossile à Grignon; les Vulselles n'ont point de byssus, et cependant ne paraissent pas se fixer par l'une de leurs valves; mais elles se logent dans certains corps sous-marins, tels que les Alcyons et les Éponges.

\section{G. CCXXX. Perne, perna, Brug.}

Anim. comprimé, ayant le manteau très prolongé en arrière, et frangé à son bord inférieur; pied très petit, avec un byssus.

Coq. cornée ou noire, lamelleuse, très aplatie, subéquivalve, inéquilatérale, très irrégulière, bâil- 
lante en avant pour le passage du byssus; charnière droite, marginale, offrant de chaque côté une série de petits sillons parallèles, transverses, non intrans, dans lesquels s'insèrent les divisions du ligament; impression musculaire subcentrale.

Toutes les espèces de ce genre sont exotiques, et la plupart de l'Inde; mais nous en connaissons une des Antilles, et une autre beaucoup plus commune qui habite les îles du Cap-Verd et les Açores. Il y en a plusieurs espèces à l'état fossile. Les Pernes se fixent aux rochers par le moyen de leur byssus, et vivent à d'assez grandes profondeurs.

\section{G. CCXXXI. Crénatule, crenatula, Lam.}

Anim. inconnu.

Coq. feuilletée, aplatie, subéquivalve, inéquilatérale, irrégulière, un peu baillante en arrière, mais sans ouverture pour le byssus; sommet antérieur; charnière linéaire, marginale, marquée de crénelures sériales, calleuses, creusées en fossettes arrondies, recevant les divisions du ligament; impression musculaire subcentrale.

Ce genre, très voisin, sans doute, des Pernes, ne renferme qu'un petit nombre d'espèces, toutes des mers de l'Inde et de l'Australasie; les Crénatules ne se fixent point au moyen d'une de leurs valves ou d'un byssus; mais elles se logent dans des corps marins, tels que les Éponges.

\section{G. CCXXXII. MartenU, malleus, Lam.}

Anim. assez comprimé, ayant le manteau prolongé en arrière, et frangé par de très petits appendices tentaculaires; pied très distinct, canaliculé, portant un byssus; appendices buccaux en forme de triangles sphériques; branchies courtes et semicirculaires.

Coq. feuilletée, noire ou cornée, subnacrée, subéquivalve, inéquilatérale, très irrégulière, souvent 
auriculée, et présentant la forme $d^{\prime} u n ~ T$; sommets antérieur's peu distans; une échancrure oblique en avant pour le passage du byssus; charnière linéaire fort longue, sans dents; une fossette conique, oblique, en partie extérieure, recevant le ligament; celui-ci triangulaire et subexterne; impression musculaire assez grande et subcentrale.

On ne connaît le genre Marteau qu'à l'état vivant, et les six espèces décrites par M. de Lamarck sont toutes des mers de l'Inde et de l'Australasie; mais nous avons caractérisé l'animal d'après une espèce que nous avons trouvée à de grandes profondeurs à la Guadeloupe et à la Martinique.

On peut diviser les Marteaux, comme le propose M. de Blainville, en trois groupes, les M. à peine auriculés, les M. uniauriculés, et les M. biauriculés.

\section{G. CCXXXIII. Gervilie, gervilia, Def.}

Anim. inconnu.

Coq. allongée, étroite, solénoïde, subéquivalve, très inéquilatérale, un peu ouverte en avant, sans doute pour le passage d'un hyssus; sommet antérieur peu distinct ; charnière offrant plusicurs dents transverses, et en arrière d'autres dents longitudinales; ligament s'insérant dans plusieurs fossettes coniques; impression musculaire un peu antérieure.

M. Defrance a établi ce genre pour des coquilles fossiles.

G. CCXXXIV. Inocéramu, inoceramus, Sow.

Anim. inconnu.

Coq. épaisse, subrégulière, subéquilatérale, inéquivalve, élargie et arrondie vers la base, pointue vers les sommets; ceux-ci obliquement recourbés, un de chaque côté; charnière fermée par une série de fossettes oblongues recevant les divisions d'un ligament; impression musculaire? 
Ce genre est établi pour des espèces encore peu counues et uniquement fossiles.

\section{G. CCXXXXV. Pulvinite, pulvinites, Def.}

Anim. inconnu.

Coq. mince, arrondie, équivalve, subéquilatérale, à sommets peu inclinés en avant; charnière composée de huit ou dix dents divergentes formant autant de fossettes; impression musculaire?

M. Defrance a établi ce genre sur une seule espèce à l'état fossile.

G. CCXXXVI. Catrlle, catillus, Brongniart.

Anim. inconnu.

Coq. à tissu fibreux, plate, très déprimée, assez mince, arrondie, subéquivalve, subéquilatérale, à sommets très distincts, quoique peu saillans; charnière droite offrant une série de petites fossettes obliques et parallèles, sans doute pour recevoir les divisions du ligament; impression musculaire?

Le genre Catille est dû à M. Brongniart. Il comprend. plusieurs coquilles fossiles, quelquefois fort grandes, difficiles à obtenir, mais généralement bien reconnaissables à leur tissu fibreux.

$4^{\text {e }}$ Famille.

\section{LES AVICULÉS.}

Anim. ayant le manteau entièrement ouvert dans toute sa circonférence, excepté le long du dos, sans tubes ni ouvertures particulières, et se prolongeant quelquefois en arrière; un pied assez petit, offrant un byssus.

Coq. souvent feuilletée, généralement assez mince, nacrée, subéquivalve; charnière sans dents, ou ne présentant que de petites dents rudimentaires; une échancrure antérieure pour le passage du byssus. 
G. CCXXXVII. Avigule, avicula, Brug.

Anim. comprimé, ayant le manteau garni sur ses bords d'une double rangée de petits filets tentaculaires très courts; pied petit, canaliculé et muni d'un byssus; bouche offrant deux paires d'appendices labiaux, et en outre des lèvres frangées.

Coq. souvent feuilletée, assez fragile, comprimée, nacrée, subéquivalve, subrégulière; sommets antérieurs et un peu surbaissés; quelquefois inégalement et obliquement auriculée; charnière droite, avec ou sans dents; ligament en partic extérieur, et contenu dans un sillon; impression postérieure fort grande, l'antérieure très petite.

$I^{\text {er }}$ S.-G. Avicules proprement dites.

Anim. comme dans la caractéristique du genre. Coq. oblique, ovale, très auriculée, surtout en arrière; présentant une ou deux petites dents rudimentaires à la charnière.

Ce sous-genre renferme plusieurs belles espèces recherchées dans les collections. M. de Lamarck en compte treize, toutes exotiques, à l'exception de l' $A$. hirundo qui est de la Méditerranée, et fort commune à Cette; il y en a aussi plusieurs espèces connues à l'état fossile. Ces coquilles se fixent aux rochers à l'aide de leur byssus.

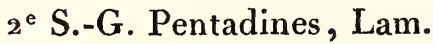

Anim. comme dans la caractéristique du genre. Coq̣. arrondie, généralement feuilletée à l'extérieur, très nacrée à l'intérieur, assez épaisse, faiblement auriculée, et d'une manière presque égale; point de dents à la charnière.

Ce second sous-genre est moins nombreux en espèces que le premier. Quelques unes d'elles fournissent une belle nacre dont l'industrie tire un grand parti; il y en a de fossiles. 


\section{$2^{\mathrm{e}}$ DIVISION. LES DIMYAIRES.}

Deux impressions musculaires à chaque valve.

5e Famile.

LES ARCACÉS, Lam.

Anim. ayant le manteau entièrement ouvert dans toute sa circonférence, excepté vers le dos, sans tubes ni ouvertures particulières, et adhérent en partie, quelquefois prolongé en arrière; un pied toujours très considérable.

Coq. généralement épaisse, régulière, équivalve, inéquilatérale, à charnière similaire toujours formée, sur chaque valve, de dents sériales, souvent lamelleuse, engrenantes, droites ou obliques; impressions musculaires presque toujours réunies par une impression paléale, très étroite et parallèle au bord de la coquille.

\section{G. CCXXXVIII. Cucullée, cucullaca, Lam.}

Anim. très épais, ayant le manteau un peu prolongé en arrière, et bordé d'une rangée irrégulière de filets tentaculaires; appendiices labiaux petits et triangulaires; pied grand, pédonculé, comprimé et fendu dans sa longueur.

Coq. épaisse, naviculaire, équivalve, inéquilatéraỉe, ventrue, à sommets écartés; charnière linéaire, droite, furmée de petites dents transverses dans la plus grande partie de sa longueur, et a ses extrémités de plusieurs autres dents ou côtes longitudinales; ligament tout-à-fait extérieur; impression musculaire antérieure formant une saillie à bord anguleux ou auriculé.

Le genre Cucullée, extrêmement voisin de celui qui le suit, renferme quelques espèces fossiles, et une seule vivante, la $C$. auriculifera, qui est des mers de l'Inde.

G. CCXXXIX. Arche, archa, Lam. Anim. plus ou moins épais, généralement assez al- 
longé; manteau se prolongeant un peu en arrière, et offrant sur ses bords une rangée de filets tenta. culaires; appendices labiaux fort petits et grêles; pied pédonculé, comprimé et fendu dans toute sa longueur.

Coq. naviculaire, assez épaisse, équivalve, inéquilatérale, allongée, plus ou moins oblique; sommets écartés et souvent un peu recourbés en avant; charnière linéaire, droite, garnie dans toute sa longueur d'une rangée nombreuse de petites dents ou lames transverses, égales et intrantes; ligament tout-à-fait extérieur.

Les animaux des Arches se fixent quelquefois par l'adhérence de leur pied, et plus souvent par le moyen d'un byssus. 11 y a un grand nombre d'espèces vivantes ou fossiles.

\section{G. CCXL. Pétoncee, pectunculus, Lam.}

Anim. arrondi plus ou moins épais, n'offrant point de filets tentaculaires au bord de son manteau; appendices labiaux très étroits; pied grand, comprimé et fendu en long.

Coq. lenticulaire, épaisse, solide, équivalve, subéquilatérale, entièrement close; sommets assez petits et plus ou moins écartés; charnière formée d'une série en ligne courbe de petites dents étroites, assez nombreuses, intrantes, souvent incomplètes sous les sommets; ligament extérieur.

Les Pétoncles vivent sur des fonds sablonneux ou vaseux à une assez grande profondeur; ils se meuvent au moyen do. pied dont l'animal se sert arec force pour se pousser. Il y en a plusieurs fossiles.

\section{G. CGXLT. Nucule, nucula, Lam.}

Anim. assez épais, subtriquètre, ayant le manteau ouvert seulement dans sa moitié inférieure, à bords entiers, denticulés dans toute la longueur du dos, 
sans prolongemens postérieurs; appendices buccaux antérieurs, assez longs, pointus, roides et appliqués l'un contre l'autre comme des espèces de mâchoires, les postérieurs également roides et̂t. verticaux; pied fort grand, mince à sa racine, élargi en un grand disque ovale dont les bords sont garnis de digitations tentaculaires.

Coq. assez épaisse, quelquefois nacrée, subtriquètre, équivalve, inéquilatérale; sommets contigus et courbés en avant; charnière formée sur chaque valve d'une série assez nombreuse de petites dents aiguës, disposées en une ligne brisée sous le sommet ; ligament en grande partie interne, court et inséré dans une petite fossette oblique de chaque valve.

Les Nucules, qui sont toutes de petites coquilles, présenTent quelques espèces fossiles.

\section{G. CCXliI. Trigonie, trigonia, Brug.}

Anim. ayant le manteau ouvert dans toute sa longueur; point de tubes postérieurs; pied fort et tranchant.

Coq. épaisse, nacrée, subtrigone, équivalve, inéquilatérale, à sommets assez petits et peu recuurbés; charnière complexe, dissemblable, la valve droite offrant deux grosses dents oblongues, divergeant du sommet, fortement sillonnées, pénétrant dans deux excavations de même forme, et également sillonnées de la valve gauche; ligament extérieur; impressions musculaires non réunies par une impression palléale.

Les caractères de l'animal nous ont été communiqués par M. Quoy, qui vient de rapporter à Paris celui de la $T$. peciinata, la seule espèce connue à l'état vivant, et qui avait été découverte par Péron à la Nouvelle-Millande. 
L'individu de MM. Quoy et Gaimard est petit, et a été retiré par la drague d'un fond de quatorze brasses.

\section{6e FAMILLE.}

\section{LES MYTILACÉS, Guv.}

Anim. ayant le manteau ouvert dans toute sa partie inférieure, et adhérent vers ses bords; une ouverture séparée, en arrière, pour les excrémens, formant très rarement un tube; le pied linguiforme, canaliculé, portant un byssus en arrière.

Coq. assez mince, généralement épidermée ou cornée, équivalve, très inéquilatérale; la charnière sans dents; le ligament linéaire; l'impression musculaire antérieure, fort petite, la postérieure assez large. Marins (le genre Moule présente seul une espèce que l'on dit d'eau douce).

\section{G. CCXLIII. Moure, mytilus, Lin. Et Modiole, Lam.}

Anin. ovale, assez épais, ayant le manteau à bords frangés autour de l'ouverture anale; bouche garnie de petits appendices labiaux triangulaires; pied linguiforme, canaliculé, portant le byssus à sa base.

Coq. assez mince, d'un tissu serré, allongée, plus ou moins ovalaire ou subtriangulaire, équivalve, très inéquilatérale, oblique, à sommets antérieurs plus ou moins courbes et arrondis; faiblement échancrée inférieurement; charnière sans dents, ou simplement avec deux dents rudimentaires; ligament linéaire, en grande partie intérieur, inséré dans un sillon étroit et allongé; impressions musculaires très écartées, l'antérieure très petite, et presque au sommet, la postérieure grande et arrondie, toutes deux réunies par une impression palléale étroite. 
On peut former deux groupes assez distincts avec les espèces de ce genre.

$$
\mathrm{x}^{\mathrm{er}} \text { groupe. }
$$

Coq. ayant le sommet tout-à-fait terminal, plus anguleux. (Moule, Lam.)

a. Coquilles sillonnées longitudinalement.

M. magellanicus, etc.

b. Coquilles lisses.

M. elongatus, etc.

$$
2^{\mathrm{e}} \text { groupe. }
$$

Coq. munie en dedans de chaque valve, et sous le sommet qui est terminal, d'une petite lame parallèle à leur bord.

M. brardii, etc.

La Moule des eaux douces du Danube fait partie de ce groupe, et présente le caractère qui le distingue d'une manière plus évidente.

$$
\text { 3e groupe. }
$$

Coq. à sommet non terminal plus arrondi. (Modiole, Lam.)

a. Coq. sillonnées longitudinalemeut.

M. sulcata, etc.

b. Coq. non sillonnées longitudinalement.

M. silicula, etc.

Les Moules se fixent au moyen de leur byssus sur les rachers, dont elles couvrent souvent la surface de leurs innombrables essaims, ou bien encore elles se logent dans les trous des pierres et des madrépores. Il y en a plusieurs espèces à l'état fossile.

G. CCXLIV. Lithonome, lithodomus, Cuv.

Anim. oblong, très allongé, épais, a vant le manteau 
prolongé et frangé en arrière; ouverture anale moyenne; bouche munie de très petits appendices triangulaires; pied linguiforme, canaliculé, peu développé et portant un byssus à sa base, pendant le jeune âge.

Coq. mince, épidermée, oblongue, très allongée, subcylindrique, arrondie en avant, plus anguleuse en arrière, non bâillante; sommet subantérieur très distinct, charnière sans dents; ligament linéaire, en majeure partie intérieur, inséré dans un sillon étroit et allongé ; impression musculaire antérieure assez grande.

Les Lithodomes ont été séparés des Moules par M. Cuvier avec plus de raison que les Modioles ne l'ont été par M. de Lamarck. Les habitudes singulières des Lithodomes et la forme de leur coquille justifient peut-être assez cette distinction générique, qui d'ailleurs a été généralement adoptée depuis. Les Lithodomes se suspendent d'abord aux rochers par le moyen de lear byssus; mais bientôt ils les percent pour s'y introduire, et $y$ forment des cavités d'ou ils ne peuvent plus sortir à cause de l'augmeniation de volume que l'âge apporte chez eux. Dans ce cas, le byssus leur devenant inutile, ne se manifeste plus. Ces coquilles sont très communes dans la Méditerranée et aux Antilles où on les trouve dans les pierres et les masses de madrépores, et quelquefois, mais bien plus rarement, dans certaines. coquilles épaisses. Il y en a à l'état fossile.

G. CGXLV. Jambonneau, pinna, Lin.

Anim. assez épais, allongé, ovalaire, ayant le manteau ouvert en arrière, où il présente quelquefois une espèce de tube garni à son ouverture de petits filets tentaculaires; bouche pourvue de deux lèvres doubles, outre deux paires d’appendices ordinaires; pied linguiforme, conique, sillonné, portant un byssus très considérable.

Coq. fibreuse, cornée, assez mince, fragile, comprinée, régulière, équivalve, longitudinale, trian- 
gulaire, pointue en avant, arrondie ou tronquée en arrière; sommet antérieur peu distinct; charnière linéaire, droite, sans dents; ligament marginal en grande partie intérieur, très étroit et serré, occupant plus de la moitié antérieure du bord dorsal de la coquille, dans une fossette étroite et allongée; impression musculaire antérieure très petite et tout-à-fait dans l'angle, la postérieure très large.

Les Jambonneaux, malgré le peu d'épaisseur de leur valve, acquièrent une très grande taille; on en trouve dans presque toutes les mers; mais la plus grande espèce habite la Méditerranée. Ces coquilles se fixent aux corps sousmarins par le moyen d'un byssus soyeux bien plus considérable que celui des autres mollusques acéphales, et elles se déplacent parfois en se servant de leur pied. On en connaît une quinzaine d'espèces vivantes, et quelques unes fossiles.

\section{$7^{\text {e FA MILLE. }}$}

LES SUBMYTILACÉS, Blainv.

Anim. ayant le manteau entièrement ouvert inférieurement, avec un orifice particulier pour l'anus, et au-dessous de celui-ci un tube incomplet pour la respiration, garni de papilles tentaculaires; pied très grand et épais, sans byssus.

Coq. plus ou moins épidermée, souvent nacrée, régulière, équivalve, inéquilatérale, à charnière variable; ligament externe; deux impressions musculaires grandes; une impression palléale, parallèle au bord de la coquille, les réunissant. Fluviatiles ou marines.

† Coq. épidermée, nacrée à l'intérieur, d'eau douce. 
G. CCXLVI. Anodonte, anodonta, Brug. Lastène et Strophite, Raf.; Dipsas, Leach.

Anim, ovale-oblong, plus ou moins allongé et épais, ayant le manteau ouvert dans toute sa moitié inférieure et en avant, adhérent, à bords épais, souvent frangés; muni d'un orifice particulier pour l'anus, et d'un tube incomplet, court, postérieur, garni de deux rangées de papilles tentaculaires, et servant à la respiration; appendices labiaux triangulaires; branchies assez longues, inégales sur un même côté; pied très grand, épais, comprimé, de forme quadrangulaire.

Coq. ovale ou arrondie, généralement assez mince, et auriculée, régulière, équivalve, inéquilatérale, non bâillante; sommet antéro-dorsal écorché; charnière sans dent, mais présentant une lame; ligament linéaire, extérieur, très allongé; impressions musculaires écartées, très distinctes.

Le genre Anodonte renferme un grand nombre d'espèces répandues sur presque tout le globe, mais particulièrement dans les deux Amériques; il y en a également plusieurs dans les Indes, et la France en compte au moins trois. Le genre Dipsas a été établi par le docteur Leach pour une espèce auriculée, et ayant une lame allongée, bien plus saillante à la charnière. Les Anodontes vivent dans les lacs, les rivières et même les petites mares d'eaux douces; elles s'enforcent en partie dóns la vase ou se meuvent à sa surface au moyen de leur pied.

G. CCXLVII. Mulette, unio, Brug. Hyrie, Castalie, Lam.; Alasmodonte, Say.; Amblémides, Uniodiés, Raf.

Anim. semblable à celui des Anodontes. Coq. de forme très variable, équivalve, inéquilatérale, assez bombée, quelquefois un peu bâillante, aurinnlée ou non; valves épaisses, rongées aux 
sommets; ceux-ci plus ou moins antérieurs; charnière formée d'une dent lamelleuse sous le ligament, et d'une double dent, comprimée, dentelée irrégulièrement sur la valve gauche, et simple sur la valve droite; ligament extérieur et allongé; impressions musculaires très écartées et peu distinctes.

Ce genre, comme le précédent, comprend un très grand nombre d'espèces, la plupart inédites ou incomplétement déterminées. L'Amérique du Nord en fournit surtout plusieurs belles devenues assez communes dans nos collections depuis les envois qui ont été faits par M. Lesueur. M. de Blainville réunit les deux geures Hyrie et Castalie de M. de Lamarck aux Mulettes, et il pense même que, par la suite, on pourra trouver des espèces qui établiront le passage des Anodontes aux Mulettes de telle manière qu'il faudra encore réunir ces deux genres; en effet, leurs animaux étant parfaitement semblables, leur distinction générique ne repose donc que sur des caractères de coquille qui peuvent disparaître au premier instant; elles habitent dans les lacs et les rivières comme les Anodontes. M. Defrance cite des espèces de Mulette à l'état fossile.

†† Coq. faiblement épidermée, non racrée à l'iutérieur, marine.

G. CCXLVIII. Cardite, cardita, Brug. Et Vénéricarde, Lam.

Anim. semblable à celui des deux genres précédens, Coq. très épaisse, solide, équivalve, souvent très inéquilatérale; sommets recourbés en avant; charnière à deux dents inégales, obliques, l'une courte, cardinale, et l'autre plus en arrière, longue, lamelleuse èt arquée; ligament allongé, subextérieur ct enfoncé; impressions musculaires assez. grandes et très distinctes; impression palléale étroite.

A l'exemple de M. de Blainville, nous réunissons aux 
Cardites les Vénéricardes; mais nous en distinguons les Cypricardes. Ces deax premiers genres, ainsi réunis, présentent un grand nombre d'especes vivantes. On en connaît aussi beaucoup à l'état fossile. M. de Blainville divise les Cardites de la manière suivante.

$$
\text { I er groupe. Mytilicardes. }
$$

Coq. allongée, un peu échancrée ou bâillante au bord inférieur; le sommet presque céphalique; le ligament caché.

C. crassi costa, etc.

$2^{e}$ groupe. Cardiocardites.

Coq. ovale, à bord inférieur presque droit ou un peu bombé, crénelé et complétement fermé.

C. ajar, etc.

$3^{\mathrm{e}}$ groupe. Vénéricardes, Lam.

Coq. presque ronde ou suborbiculaire, à bord inférieur arrondi, denticulé, de plus en plus équilatérale; les deux dents plus courtes et plus obliques.

C. australis, etc.

\section{G. CCXLIX, Cypricarde, Lam.}

Anim. semblable à celui des deux genres précédens. Coq. assez épaisse, solide, équivalve, très inéquilatérale, allongée; sommets un peu recourbés en avant; charnière munie de deux dents cardinales, courtes, divergentes, sous les sommets, et d'une dent lamelleuse latérale se prolongeant en arrière ; ligament très long, en grande partie antérieur; impressions musculaires assez petites et distantes; impression palléale quelquefois un peu rentrée postérieurement.

Ce genre renferme un petit nombre d'espèces vivantes et fossiles. 
80 FAMILLE.

\section{LES CAMACÉS, Lam.}

Anim. ayant le manteau ouvert à sa partie inférieure, seulement pour le passage du pied; les bords du manteau adhérens et finement frangés, réunis en arrière par une bande transverse percée de deux orifices, l'un pour les déjections excrémentitielles, l'autre pour la respiration.

Coq. irrégulière ou régulière, inéquilatérale, inéquivalve ou non; la charnière avec une dent grossière, ou sans dent; l'impression palléale peu apparente. Marins ou d'eau douce.

† Coq. irrégulière, inéquivalve.

G. CCL. Éthérie, etheria, Lam.

Anim. inconnu.

Coq. adhérente, épaisse, nacrée, très irrégulière, inéquivalve, inéquilatérale ; sommets courts, épais, peu distincts, confondus dans une sorte de talon; charnière sans dents, irrégulière, ondée, calleuse; ligament longitudinal, tortueux, extérieur, pénétrant en pointe dans l'intérieur de la coquille; impressions musculaires ovales, irrégulières; l'une supérieure et postérieure, l'autre inférieure et antérieure; l'impression palléale étroite et petite.

Ce genre précieux par sa rareté, et qui n'est répandu que depuis le voyage en Egypte de M. Caillaud, appartient aux eaux douces des fleuves, particulièrement d'Afrique et de Madagascar.

\section{G. CCLI. CAMe, chama, Lin.}

Anim. plus ou moins orbiculaire et épais, ayant le 
manteau très peu ouvert inférieurement; pied petit, coudé; branchies inégales, sur un même côté, la supérieure étant très courle; deux ouvertures postérieures petites, ayant quelquefois leurs bords saillans et prêts à commencer des tubes.

Coq. épaisse, solide, adhérente, irrégulière, inéquivalve, inéquilatérale, ayant les sommets inégaux plus ou moins contournés en spirale, et distincts; charnière composée d'une seule dent lamelleuse, épaisse, oblique, subcrénelée, s'articulant avec un sillon de la valve opposée; ligament extérieur et enfoncé; impressions musculaires assez grandes.

Le genre Came renferme des espèces généralement feuilletées ou spinifères, qui s'attachent aux corps sous marins à d'assez grandes profondeurs, et se réunissent quelquefois par groupes nombreux. M. de Lamarck les divise de la manière suivante.

1 er groupe.

Coq. tournant de gauche à droite.

C. gryphoides, etc.

$2^{\mathrm{e}}$ groupe.

Coq. tournant de droite à gauche.

C. arcinella, etc.

Les Cames sont en général des mers chaudes. On en connaît plusieurs à l'état fossile.

\section{G. CCLII. Dicérate, diceras, Lam.}

Anim. inconnu.

Coq. irrégulière, inéquivalve, inéquilatérale, adhérente, à sommets coniques, très grands, divergens, presque régulièrement contournés en spirale; charnière composée d'une grosse dent concave, à la 
plus grande valve; impressions musculaires dis. tinctes; ligament inconnu.

Ce genre est très imparfaitement connu sans doute; mais on peut croire qu'il est très-voisin des Cames. Une seule espèce lui sert de type, c'est la D. arietina, coquille fossile du mont Salève.

\section{G. CCLIII. Caprine, caprina, Dorb.}

Anim. inconnu.

Coq. irrégulière, inéquivalve, inéquilatérale, à sommets coniques, écartés, plus ou moins inégalement prolongés et roulés sur deux plans opposés ; charnière et ligamens inconnus; cavité des valves divisée par une cloison en deux loges coniques, inégales; deux impressions musculaires situées dans de petites cavités, l'une antérieure et inférieure, l'autre supérienre et postérieure.

Le genre Caprine, créé par M. Dorbigny père pour des coquilles fossiles de la Charente-Inférieure, est encore si peu connu, et ce savant lui-même avait si peu de notion à son sujet, lorsqu'il en a publié la première description dans les Mémoires du Muséum, qu'il a été généralement laissé de côté par les naturalistes qui ont écrit depuis, ou bien confondu par eux avec les Dicérates que l'on ne connaît guère mieux. Nous avons vu dans la belle collection de M. Dorbigny une grande quantité de ces coquilles, et leur examen nous donne lieu de croire qu'elles diffèrent assez des Dicérates pour former un genre à part; nous en avons distingué plusieurs espèces, les unes ayant une dimension de plus d'un pied de diamètre, et d'autres infiniment plus petites; toutes offrant plus ou moins complétement les caractères génériques que nous venons d'indiquer. M. Dorbigny en compte actnellement quinze à vingt espèces; mais nous peusons que, dans ce nombre, quelques coquilles, munis d'une valve operculaire, recouvrant une 
antre valve beaucoup plus grande, sortent des limites in. diquées par les caractères des Caprines, et qu'elles pourront constituer peut-être d'autres genres voisins de celui-ci. Il serait donc vivement à désirer que ce naturaliste, dont la science apprécie si bien les lumières et les recherches, fit, au sujet de ces intéressantes coquilles, un nouveau travail qu'il pourrait facilement étendre davantage au moyen des nombreuses découvertes qu'il a faites depuis quelques années; rien ne s'opposerait certainement alors à ce qu'un tel genre ne fût unanimement adopté.

Le genre Ichthyosarcolite, qui a toujours été classé avec les coquilles des Céphalopodes, pourrait bien appartenir à une coquille bivalve voisine des Caprines. M. Deshayes nous a communiqué la même idée.

†† Coquille régulière, équivalve.

G. CCLIV. Isocarde, isocardia, Lam.

Anim. plus ou moins globuleux, ayant les bords du manteau garnis de papilles tentaculaires très fines; ceux-ci laissant une ouverture assez grande entre eux à la partie inférieure, et réunis postérieurement par une bande transverse, mince, percée de deux orifices entourés de papilles, l'un pour l'anus, et l'autre, plus inférieur, pour la respiration; pied de grandeur moyenne, et tranchant.

Coq. quelquefois épidermée, très bombée, globuleuse, cordiforme, équivalve, inéquilatérale ; sommets écartés, divergens fortement, recourbés en avant et en dehors, un peu spirales; charnière composée de deux dents cardinales aplaties, intrantes, dont une s'enfonçe en se courbant sous le crochet, et d'une autre dent lamelleuse écartée en arrière du ligament; celui-ci extérieur, fourchu à l'une de ses extrémités; impressions musculaires très distantes et assez, petites. 
Ce genre ne renferme qu'un très petit nombre d'espèces à l'état vivant; on en compte plusieurs à l'état fossile.

\section{G. CCLV. Tridacie, tridacna, Lam.}

Anim. épais, ayant les bords du manteau adhérens, très renflés, et réunis dans presque toute sa circonférence, de manière à ne laisser que trois ouvertures assez petites; l'une qui est en bas pour la sortie du pied, l'autre en arrière et en haut pour la respiration, et la troisième, qui est la plus petite, au milieu du hord dorsal; appendices labiaux subfiliformes; bouche très petite; branchies allongées, étroites, inégales sur un même côté, et réunies presque dans toute leur longueur; muscle adducteur postérieur, étant médian et presque dorsal, l'antérieur très petit; pied muni d'un byssus composé de fibres tendineuses.

Coq. très épaisse, solide, régulière, équivalve, inéquilatérale, subtriangulaire, à lunule bâillante', les sommets un peu inclinés en arrière; charnière en avant d'eux, formée de deux dents, et une lame de la valve gauche correspondant à une dent et deux lames de la valve droite; ligament allongé, extérieur et antérieur; impression musculaire postérieure, centrale et bifide, se confondant à sa partie inférieure avec le milieu de l'impression palléale; l'antérieure très petite, ne se distinguant que très confusément à l'extrémité de l'impression palléale; celle - ci large, rubanée, parallèle au bord de la coquille, et s'élevant de chaque côté.

M. de Blainville a observé que les Tridacnes adultes ont la lunule fermée, et que, par conséquent, elles ne diffèrent pas des Hippopes; il en conclut aussi que les Tridacnes u'adbèreat pas toujours. Nos recherches à l'île de Bourbon et à Madagascar, où ces coquilles sont extrêmement communes, ne nous ayant pas convaincus de ce fait, nous conserverons le genre Hippope jusqu'à nouvel exa- 
men. L'animal des 'Tridacnes est placé dans la coquille d'une manière un peu différeute des autres mollusques Lamellibranches; par un certain retournement, il se trouve que ses différentes parties n'ont pas la correspondance ordinaire, et M. de Blainville pense que cela peut être dû̀ sa suspension, opinion qui paraît extrêmement probable. Ce genre ne renferme, d'après M. de Lamarck, que six espèces toutes vivantes et des mers de l'Inde.

\section{G. CCLVI. Hippore, hippopus, Lam.}

Anim. semblable à celui des tridacnes?

Coq. très épaisse, solide, régulière, équivalve, subéquilatérale, à lunule très grande, aplatie et close; sommets grands, recourbés en avant; charnière formée de deux grosses dents comprimées, et une lame imparfaite de la valve gauche correspondant à une pareille dent plus grosse et deux lames également imparfaites de la valve droite; ligament allongé, antérieur et extérieur; impression musculaire postérieure, centrale, arrondie, non bifide, se confondant inférieurement avec l'impression palléale; impression antérieure à peine distincte, à l'extrémité de l'impression palléale; celie-ci longue, rubanée, parallèle au bord de la coquille.

Une seule espèce vivante, et qui vient des mers de l'Inde, compose ce genre.

\section{$9^{\text {e Famille. }}$}

\section{LES CONCHACÉS, Blainv.}

Anim. ayant le manteau fermé, muni d'une ouverture assez grande, antéro-inférieure, pour le passage d'un pied, et présentant deux tubes postérieurs plus ou moins allongés, extensibles, réunis ou séparés dans leur longueur, servant, l'inférieur à la respiration, et le supérieur aux déjections excrémentitielles.

Coq. équivalve, généralement régulière, rarement 
bâillante; sommets toujours plus ou moins recourbés en avant; charnière presque toujours par engrenage ; ligament court et bombé, intérieur ou extérieur; impressions musculaires très distinctes, réunies par une impression palléale plus ou moins excavée postérieurement.

Marins, rarement d'eau douce.

Cette famille est, à très peu de chose près, telle que M. de Blainville l'a formée; nous n'avons fait qu'y ajouter le genre nouveau créé par M. Charles Des Moulins sous le nom de Gratelupie pour une coquille fossile-roisine des Donaces et des 'Tellines, ainsi que l'Iridine qui, d'après les observations de M. Deshayes, ne peut plus rester arec les Submytilacés. Nous avons aussi rétabli, comme genre, quelques mollusques présentés comme divisions de genre par M. de Blainville.

† Charnière linéaire sans engrenage; d'eau douce.

\section{G. CCLVII. Iridine, iridina, Lam.}

Anim. allongé, étroit, assez épais du côté du dos, plus mince vers son bord inférieur; manteau mince, terminé antérieurement par un limbe épaissi, ouvert depuis le muscle antérieur jusque vers les deux tiers de son bord inférieur pour le passage du pied; bords du manteau réunis dans toute la partie postérieure où naissent deux tubes courts et inégaux; pied très comprimé et tranchant.

Coq. épidermée à l'extérieur, nacrée ou irisée à l'intérieur, assez épaisse, solide, ovale ou oblongue, allongée, inauriculée, équivalve, inéquilatérale, le côté antérieur étant plus court que le postérieur, un peu bâillante en avant comme en arrière; sommets petits et peu saillans, faiblement inclinés; charnière très longue, linéaire, atténuée vers le milieu, souvent comme crénelée dans toute sa longueur; ligament très long, marginal, extérieur; impressions musculaires très distinctes. 
Ce genre avait toujours été confondu avec les Anodontes, parce qu'en effet leurs coquilles diffèrent très peu; mais M. Deshayes, ayant eu un individu conservé dans l'esprit de vin, et rapporté du Nil par M. Caillaud, a reconnu que ce mollusque appartient à une autre famille que les Anodontes, puisqu'il a postérieurement des tubes, tandis que les Anodontes en sont dépourvues; il nous fallait donc placer ce genre dans les Conchacés, d'abord parce que l'ouverture antérieure de son manteau est encore très grande, ensuite parce que sa coquille ne permettait pas de l'éloigner beaucoup des Anodontes. On doit sans doute rapporter à cette famille et à la même division les Anodontes, qui montrent également des tubes à la partie postérieure du manteau, et qui, par conséquent, ne peuvent plus rester dans le genre.

Le genre Iridine ne renferme encore, à ce qu'il paraît, qu'une seule espèce dans laquelle on distingue deux variétés, l'une crénelée à sa charnière et l'autre non crénelée. Elles sont du Nil et des fleuves de la Chine. C'est une coquille encore rare.

$\dagger †$ Charnière par engrenage.

$a$. Coquilles régulières; des dents latérales écartées.

r. Marines.

\section{G. CCLVIII. Bucarde, cardium, Lin.}

Anim. très bombé, ayant le manteau amplement ouvert, inférieurement bordé de papilles tentaculaires; pied très grand, cylindrique, dirigé en avant et coudé dans son milieu; tubes réunis, assez courts, et quelquefois inégaux, ayant leurs ouvertures bordées de papilles; bouche transverse, infundibuliforme, avec de petits appendices triangulaires; branchies courtes, inégales de chaque côté, réunies sur une même ligne.

Coq. bombée, souvent subglobuleuse, subcordiforme, équivalve, à côtes radiaires; bords des valves dentés ou plissés; sommets peu recourbés 
en avant; charnière formée de quatre dents sur chaque valve, deux cardinales, obliques, et deux latérales écartées; ligament postérieur et très court.

Les Bucardes sont des coquilles extrêmement répandues; elles offrent un grand nombre d'espèces tant à l'état frais qu'à l'état fossile.

\section{G. CCLIX. Hémicarne, hemicardium, Cuv.}

Anim. très épais, court, subtrigone, ayant le manteau assez largement ouvert pour le passage d'un pied moyen et coudé; tubes courts, réunis dans presque toute leur longueur; branchies courtes et très inégales de chaque côté.

Coq. subtrigone, cordiforme, à côtes radiaires; très aplatie en avant, carénée de chaque côté, et plus ou moins renflée en arrière; charnière composée de quatre dents sur chaque valve, deux cardinales et deux latérales écartées, toutes assez rapprochées; le ligament très court.

Ce genre, établi par M. Cuvier aux dépens des Bucardes, mérite certainement d'être conservé, car, comme l'observe l'auteur du Règne animal, il est impossible que le mollusque qui forme cette coquille ne suit pas modifié en raison de sa configuration singulière, et c'est ce dont nous avons pu nous convaincre sur plusieurs individus du Cardium Cardissa que nous avons observés vivans.

Ce genre renferme trois à quatre espèces vivantes toutes exotiques, et plusieurs espèces fossiles.

\section{G. CCLX. Capse, capsa, Brug.}

Anim. ayant le manteau assez largement ouvert au bord antéro-inférieur pour le passage d'un pied comprimé et très largø; tubes séparés et asser 
longs, avec des papilles tentaculaires à leurs orifices.

Coq. subtrigone, épidermée, assez bombée, équivalve, inéquilatérale, plus longue que haute; charnière formée de deux dents assez minces sur la valve gauche, et d'une dent bifide et intranté sur celle de droite; ligament extérieur et postérieur; impressions musculaires assez grandes, ovales et distantes, réunies par une impression palléale, étroite, peu marquée, et très excavée en arrière.

Ce genre, peu nombreux et tout exotique, est tellement voisin des Donaces, que l'on pourrait, à l'exemple de M. de Blaiuville, n'en faire qu'un des deux. Ces coquilles se tiennent daus le sable à peu de profondeur, le bord postérieur tourné vers le haut pour faciliter l'entrée de l'eau nécessaire à la respiration.

\section{G. CCLXI. Donace, donax, Lin.}

Anim. un peu comprimé, plus ou moins triangulaire, ayant le manteau bordé d'appendices tentaculaires; appendices labiaux, grands; bouche petite; branchies très inégales du même côté; pied comprimé, tranchant, anguleux; tubes séparés et allongés, rentrant dans un sinus du manteau. Coq. plus ou moins triangulaire et comprimée, toujours plus longue que haute, régulière, équivalve, très inéquilatérale, le côté postérieur étant plus court que l'antérieur; sommets peu proéminens et presque verticaux; charnière composée de deux dents cardinales sur les deux valves ou sur une seule; une ou deux dents latérales plus ou moins écartées; ligament extérieur, court et bombé; impressions musculaires arrondies, réunies par une impression palléale, étroite et fortement excavée en arrière. 
Ce genre renferme une trentaine d'espèces vivantes que l'on pourrait diviser en deux groupes au moyen de l'aplatissement que l'on remarque quelquefois à leur bord postérieur. Elles peuvent encore se subdiviser par la considération de la charnière qui varie beaucoup. Il y en a à l'état fossile.

\section{G. CCLXII. Gratelupie, gratelupia, Charles Des Moulins.}

Anim. inconnu.

Coq. subtrigone, équivalve, régulière, presque équilatérale, un peu atténuée à sa partie postérieure, et présentant an bord postéro-inférieur une légère sinuosité; crochets très petits, peu saillans, à peine inclinés en avant; charnière composée sur chaque valve de trois dents cardinales divergentes, et de trois à six dents cardini-sériales, convergentes vers les sommets, lamelleuses, à bords finement dentelés, et situées un peu en arrière du sommet, sous le ligament; une seule dent latérale, antérieure, sous la lunule, à la valve gauche, correspondant avec une fossette pareillement située à la valve droite; ligament extérieur, long, bombé, . dépassant les dents sériales; impressions musculaires à peu près égales, ovales, réunies par une impression palléale largement et très profondément excavée en arrière.

C'est à M. Charles Des Irioulins que l'on doit l'établissement de ce genre pour une coquille de son département confondue autrefois par M. de Basterot, avec les Donaces sous le nom de $D$. irregularis. M. Des Moulins, étant parvenu à s'en procurer de beaux échantillous, a pu donner une description exacte et détaillée de ses caractères, et ceux qu'il a bien voulu nous communiquer ne nous laissent aucun doute sur la nécessité d'une distinction générique. La Gratelupie appartient aux dépôts marins de Mériguac, et ne présente encore qu'une seule espèce, $\dot{G}$. donaciformis.. 


\section{G. CCLXIII. Trelive, tellina, Linné. Et Tellinide, Lam.}

Anim. généralement très comprimé, assez allongé, muni d'un manteau médiocrement ouvert à sa partie antéro-inférieure, et hordé d'appendices tentaculaires; branchies inégales de chaque côté; pied très comprimé, tranchant, et pointu en avant; tubes fort allongés, séparés, et rentrant dans un repli du manteau.

Coq. généralement allongée et très comprimée, équivalve, régulière, quelquefois faiblement inéquilatérale, le côté antérieur n'étant pas toujours beaucoup plus long que le côté postérieur, qui se montre assez souvent anguleux, avec un pli flexueux et irrégulier à son bord inférieur; sommets très petits; charnière composée d'une ou deux dents cardinales, et de deux dents latérales souvent écartées, avec une fossette à leur base, sur chaque valve; ligament postérieur, bombé et allongé; un second ligament très petit près du sommet; impressions musculaires arrondies; impression palléale étroite, et très profondément excavée.

Les Tellines, si analogues aux Donaces, vivent, comme elles, enfoncées dans le sable; elles sont de toutes les mers, mais particulièrement des climats chauds; leur nombre est, sans doute, très considérable, car M. de Lamarck en cite. déjà cinquante-cinq à l'état virant, en y comprenant l'espèce dont il a formé le genre Tellinide, mais qu'à l'exemple de $M$. de Blainville, nous ne croyons pas devoir maintenir. On en connait aussi plusieurs à l'état fossile.

\section{G. CCLXIV. Lucre e, lucina, Brug. Et Loripèdes, Poli.}

Anim. plus ou moins épais, ayant les bords du manteau finement frangés; tubes courts et réunis, rcn. 
trant dans un repli du manteau; pied allongé et cylind rique.

Coq. comprimée, orbiculaire, régulière, équivalve, subéquilatérale; sommets assez proéminens, et uu peu inclinés en avant; charnière formée de deux dents cardinales divergentes, peu prononcées, et quelquefois de deux autres dents latérales écartées, avec une fossette à la base; ligament postérieur et assez allongé; impressions musculaires allongées, réunies par une impression palléale sans excavation.

Ce genre renferme un bon nombre d'espèces, que l'on peut diviser de la manière suivante àvec M. de Blainville.

$$
\text { I er groupe. }
$$

Coq. lenticulaire, striée concentriquement; la lunule et le corselet indiqués en relief; les dents de la charnière variables et quelquefois nulles.

L. jamaicensis, etc.

$$
2^{\text {e }} \text { groupe. }
$$

Coq. de même forme; la lunule et le corselet non saillans.

L. lactea, etc.

$$
\text { 3e groupe. }
$$

Coq. lenticulaire, pectinée ou rayonnée du sommet à la base.

L. scabra, etc.

M. de Blainville comprend encore dans ce genre l'Amphidesme de M. de Lamarck et le genre Corbeille de M. Cuvier. Le nombre des coquilles fossiles qui se rapportent aux Lucines est assez grand.

\section{G. GCLXV. Corbeille, corbis, Cuv.}

Anim. inconnu, mais probablement peu différent de celui des Lucines.

Coq. épaisse, très solide, bombée, un peu plus 
longue que haute, équivalve, subéquilatérale, ayant des stries longitudinales croisées par des stries divergentes du sommet; sommets en opposition et courbés en dedans; charnière composée de deux dents cardinales et deux dents latérales lamelleuses; impressions musculaires ovales, réunies par une impression palléale non excavée en arrière.

Une seule espèce vivante, la $C$. fimbriata, qui est des mers de l'Inde, forme ce genre. C'est une jolie coquille fort recherchée dans les collections. Il y en a aussi de fossiles.

G. CCLXVI. Amphidesme, amphidesma, Lam.

Anim. inconnu.

Coq. subovale ou arrondie, peu épaisse, plus longue que haute, inéquilatérale, quelquefois un peu bâillante; charnière ayant une ou deux dents cardinales, et quelquefois des dents latérales plus ou moins saillantes; ligament double, un externe, court, un autre interne, fixé dans une fossette étroite de la charnière.

Ce genre, confondu par M. de Blainville avec les Lucines, contieut plusieurs espèces parmi lesquelles il en est de nos côtes.

\section{G. CCLXVII. Cyprine, cyprina, Lam.}

Anim. épais, ovale, ayant le manteau muni en arrière de deux tubes courts et séparés, et en avant d'une ouverture pour le passage d'un pied sécuriforme. Coq. légèrement épidermée, épaisse, solide, subcordiforme, faiblement striée longitudinalement, régulière, équivalve, inéquilatérale, ayant les sommets très rapprochés et fortement recourbés en avant; charnière très épaisse, formée de trois dents cardinales inégales, peu convergentes, et d'une dent latérale écartée, quelquefois obsolite; 
ligament très épais, extérieur, bombé, s'enfoncant en partie sous les sommets; callosités nymphales grandes, arquées, terminées par une fossette; impressions musculaires ovales, réunies par une impression palléale très peu ou point excavée en arrière.

Ce genre ne renferme encore qu'une seule espèce vivante, la $C$. islendica, très peu connue dans les collections; mais on en cite davantage à l'état fossile.

\section{G. CCLXVIII. MAGTre, mactra, Lam.}

Anim. ovale, assez épais, ayant les bords du manteau épaissis et simples, munis en arrière de deux tubes peu allongés et réunis; bouche petite; appendices labiaux étroits et pointus; lames branchiales petites et presque égales; pied ovale, tranchant, très long et anguleux.

Coq. généralement assez mince, et quelquefois épidermée, subtrigone, équivalve, régulière, presque équilatérale, et quelquefois un peu bâillante en arrière; sommets assez proéminens, très faiblement courbés en avant; charnière composée d'une dent cardinale pliée en toit, en avant d'une fossette plus ou moins triangulaire pour l'insertion du ligament intérieur sur chaque valve, et de deux dents lamelléuses, latérales, peu écartées, l'une en avant et l'autre en arrière, sur la valve gauche, correspondant à quatre dents lamelleuses, pareillement situées sur la valve droite; deux ligamens, l'un extérieur très petit, l'autre au-dessous, toutà-fait intérieur, gros et triangulaire; impression musculaire antérieure, ovale, un peu allongée et arquée, la postérieure presque circulaire, toutes deux réunies par une impression palléale, étroite, dont l'excavation est assez profonde et arrondie.

Ce genre renferme un assez grand nombre de coquilles rivantes; les unes appartiennent aux mers froides et les 
autres, au contraire, aux climats chauds; elles habitent sur les plages sablonneuses où elles s'enfoncent à une petite profondeur. Il y en a aussi de fossiles.

\section{G. CCLXIX. Ényarne, erycina.}

Anim. inconnu.

Coq. subtrigone, un peu plus longue que haute, régulière, équivalve, inéquilatérale, rarement bâillante; sommets très distincts et fort peu inclinés en avant; charnière composée de deux dents cardinales inégales, convergentes au sommet, comprenant une fossette entre elles, sur chaque valve, et de deux dents latérales oblongues, comprimées, sur une valve, correspondant chacune à deux dents pareilles sur la valve opposée; ligament intérieur, court et triangulaire, occupant la fossette; impressions musculaires ovales.

Ce geure se compose particulièrement d'espèces fossiles, mais quelques unes d'elles semblent devoir en être écartées. On ne conuaît à l'état frais que l'E. cardioides, qui a été recueillie sur le sable au port du Roi-George.

2. D'eau douce.

G. CCLXX. Cychade, cyclas, Lam.

Anim. épais, ayant un manteau à bords simples, muni de tubes courts et réunis; pied large, comprimé à sa base, et terminé par une sorte d'appendice. Coq. épidermée, mince, quelquefois demi-transparente, ovale, très bombée, équivalve, inéquilatérale; sommets très rapprochés et un peu tournés en avant; charnière composée de dents cardinales très petites, quelquefois presque nulles, tantôt deux. sur chaque valve, dont une pliée en deux, tantôt une seule pliée ou lobée sur une valve, et deux sur l'autre; deux dents latérales écartées, lamelliformes, avec une fossette à la base; ligament extérieur, postérieur et bombé; deux impres- 
sions musculaires réunies par une impression palléale non excavée.

M. de Lamarck rapporte onze espèces à ce genre; mais il y en a davantage. Plusieurs d'entre elles sont de nos rivières ou de nos ruisseaux, et s'y enfoncent dans la vase. On en connaît deux fossiles.

\section{G. CCLXXI. Cyrène, cyrena, Lam. Et Corbicula, Megerle.}

Anim. inconnu.

Coq. épidermée, épaisse, solide, subtrigone ou suborbiculaire, ventrue, équivalve, inéquilatérale ; sommets écorchés; charnière composée sur chaque valve de trois dents, dont les deux postérieures sont bifides, et de dents latérales presque toujours au nombre de deux, dont une souvent est rapprochée du sommet; ligament extérieur et postérieur; impressions musculaires ovales, réunies par une impression palléale sans excavation.

Les Cyrènes sont toutes des coquilles exatiques qui habitent les fleuves des pays chauds, mais particulièrement, à ce qu'il parait, de l'Asie. M. de Lamarck en forme deux groupes.

$$
{ }^{\mathrm{er}} \text { groupe. }
$$

Coq. ayant les dents latérales dentelées. (Corbicule.)

C. trigonella, etc.

$$
2^{e} \text { groupe. }
$$

Coq. ayant les dents latérales entières.

C. depressa, etc.

Il y en a de fossiles.

G. CCL,XXII. Galathée, galathea, Brug. Égérie, de Roissy; Potamophile, Sow.

Anim. inconnu.

Coq. épidermée, subtrigone, équivalve, presque équi- 
latérale, un peu atténuée en avant, où son bord inférieur est faiblement sinueux; sommets assez. proéminens, presque droits; charnière composée de dents cardinales sillonnées, deux sur une valve, convergentes au sommet, et trois sur l'autre, celle du milieu étant plus grosse, calleuse et avancée; dents latérales écartées; ligament extérieur et postérieur, saillant, court et bombé; impressions musculaires très visibles.

Une seule espèce, le G. radiata, qui vient, dit-on, des rivières de l'île de Ceylan, compose ce genre, qu'il conviendrait peut-être de réunir aux Cyrènes.

b. Coquilles régulières; point de dents latérales écartées; toutes de mer.

\section{G. CCLXXIII. Crassatelle, crassatella.}

Anim. inconnu.

Coq. épaisse, suborbiculaire ou subtrigone, un peu plus longue que haute, striée longitudinalement, régulière, équivalve, inéquilatérale; sommets proéminens et courbés en avant; les deux valves bien closes; charnière épaisse, fort large, composée de deux dents cardinales, divergentes, séparées par une fossette assez grande; ligament presque antérieur, et placé dans cette fossette; impressions musculaires arrondies, réunies par une impression palléale non échancrée postérieurement.

Les Crassatelles, à l'état frais, sont des coquilles peu répandues encore dans les collections. Il y en a plusieurs espèces toutes exotiques, et en grande partie des mers de la Nouvelle-Hollande. Nous n'en possédons, en France, que de fossiles.

G. CCLXXIV. Astarté, astarte, Sow. C'rassine, Lam.; Nicania, Leach.

Anim. inconnu, mais probablement semblable à celui des Vénus. 
Coq. assez épaisse, solide, suborbiculaire ou subtrigone, un peu plus longue que haute, équivalve, inéquilatérale; sommets proéminens, en pointe mousse; charnière composée de deux grosses dents divergentes sur une valve, et de deux dents très inégales sur l'autre; ligament extérieur, un peu allongé et bombé; impressions musculaires arrondies, et réunies par une impression palléale non excavée.

Ce genre ne diffère des Crassatelles que par son ligament ; aussi M. de Blainville n'a pas cru devoir le conserver, et il le place de préférence avec les Vénus. Une seule espèce vivante, et des côtes d'Angleterre, en est le type, c'est l' $A$. danmoniensis. On doit à M. de la Jonkaire une monographie des espèces fossiles qu'il élève à seize. Comme ce naturaliste, nous avons adopté la dénomination générique de M. Sowerby, parce qu'elle avait été affectée à des espèces fossiles de ce genre avant que M. de Lamarck n'eût établi pour l'espèce vivante, celle de Crassine.

\section{G. CCLXXV. Vénus, venus, Lin. Vénus et Cythérée, Lam.}

Anim. ovale, asscz épais, ayant les bord́s du manteau onduleux et munis d'une rangée de cirrhes tentaculaires; tubes plus ou moins allongés, et rarement séparés; bouche petite; appendices labiaux petits; branchies larges; pied grand, comprimé, tranchant, un peu variable.

Coq. en général un peu comprimée, solide, régulière, équivalve, inéquilatérale, non bâillante, très souvent ornée de côtes longitudinales, mais rarement de rayons transverses; sommets bien distincts et un peu courbés en avant; charnière composée de deux à quatre dents cardinales réunies sous le sommet; ligament épais, extérieur et bombé; impressions musculaires plus ou moins arrondies, réunies par une impression palléale plus ou moins excavée en arrière. 
Les coquilles que, à l'exemple de M. Cuvier et de M. de Blainville, nous rapportons aux Yénus, ont été partagées en deux genres par M. de Lamarck, les Vénus et les Cythérées; mais cette distinction ne reposant que sur de très faibles caractères de la coquille qui ne sont même pas toujours complétement justifiés, il se trouve que les limites de ces genres ne sont pas, dans quelques cas, très faciles à saisir.

Les groupes proposés par $M$. de Blainville suffisent pour le classement des espèces qui s'élèvent à plus de cent cinquante, sans y comprendre celles qui sont fossiles, et qui sont presqu'aussi nombreuses; nous n'avons retranché de ces groupes que celui qui renfermait l'Astarté de Sowerby, parce que nous pensons que ce genre peut être conservé.

Les Vénus vivent dans le sable, et on en trouve dans toutes les mers; plusieurs d'entre elles sont des coquilles rares, fort agréables à l'œil, et par conséquent très recherchées dans les collections.

$$
\mathrm{I}^{\mathrm{re}} \text { section. G. Cythérée, Lam. }
$$

La dent médiane profondément divisée en deux; l'antérieure plus avancée.

$$
I^{\text {er }} \text { groupe. }
$$

Coq. mince, triangulaire, bombée, à sommets très marqués; les bords tranchans, sans lunule distincte. (V. Mactroïdes. )

V. lata, etc.

$$
2^{\circ} \text { groupe. }
$$

Coq. épaisse, subtrigone; les bords du corselet carénés, sans lunule distincte.

$V$. petechialis, etc.

$$
3^{\mathrm{e}} \text { groupe. }
$$

Coq. lenticulaire, à stries concentriques, sans dent antérieure sous la lunule qui est très enfoncée; la ligule palléale profondément et anguleusement excavée en arrière; le pied de l'animal semi-lunaire. (G. Arthémis, Poli.)

V. exoleta, etc. 


\section{$4^{\text {e }}$ groupe.}

Coq. lenticulaire, radiée ou subpectinée, sans dent latérale postérieure; la lunule et le ligament très enfoncés; l'empreinte musculaire antérieure, étroite et descendante; la ligule marginale peu marquée et nou rentrée postérieurement. (V. Lucinoïdes.)

$\boldsymbol{V}$. tigerrina, etc.

$$
5^{\text {e }} \text { groupe. }
$$

Coq. épaisse, solide, plus ou moins comprimée, ovale, côtelée, pectinée sur les bords; les impressions musculaires réunies par une large ligule non sinueuse.

$V$. pectinata, etc.

$$
\text { 6* groupe. }
$$

Coq. épaisse, solide, subtrigone, striée longitudinalement, les empreintes réunies par une ligule étroite non sinueuse.

V. crassa, etc.

$$
7^{e} \text { groupe. }
$$

Coq. épaisse, solide, à peu près lisse, ou ovale-allongée, de couleur radiée ou litturée, l'impressiou abdominale formant en arrière une excavation assez profonde. (Les Mérétrices.)

$V$. chione, etc.

$$
2^{e} \text { section. G. Vénus, Laun. }
$$

La dent médiane bifide, ou trois deuts cardinales seulement.

$$
8^{e} \text { groupe. }
$$

Coq. de forme allongée, subrhomboïdale, striée, à bord non denticulé; les trois dents de la charnière très rapprochéés et très faibles.

$V$. decussata, etc.

$$
9^{e} \text { groupe. }
$$

Coq. subrhounboïdale, profondément treillisée; les 
dents très épaisses, le ligament entièrement caché; les crochets très marqués; le bord denticulé.

$V$. corbis, etc.

\section{0 ${ }^{\circ}$ groupe.}

Coq. épaisse, solide, orbiculaire ou suborbiculaire, arec des stries ou mieux des lames concentriques; les dents fort épaisses; le bord denticulé.

$V$. puerpera, etc.

$$
\text { I I e groupe. }
$$

Coq. cardioïde ou radiée du sommet à la base, épaisse, solide.

$V$. granulata, etc.

$$
12^{\mathrm{e}} \text { groupe. }
$$

Coq. triquètre, cunéiforme, épaisse, solide, striée longitudinalement, denticulée; les bords du corselet carénés; deux grosses dents obliques à la charnière; les tubes de l'animal fort courts et distincts. (G. 'Triquètre, Blainv.)

$V$. flexuosa, etc.

$$
13 \mathrm{e} \text { groupe. }
$$

Coq. solide, cordiforme, comprimée, à sillons longitudinaux; bords denticulés; dents épaisses, fort peu saillantes; le corselet long et étroit.

$\boldsymbol{V}$. casina, etc.

$$
14^{\mathrm{e}} \text { groupe. }
$$

Coq. épidermée, striée, comprimée, ovale; les sommets peu proéminens; deux dents bifides sur la valve droite, et une seule entière sur la gauche. (G. Macome, Leach.)

$V$. tenuis, etc.

Nous avons suivi dans notre collection, pour les Vénus, la classification que nous venons de faire connaître, et nous avons pu juger, par conséquent, de la valeur des caractères sur lesquels elle est fondée; mais nous engageons fortement les voyageurs qui se livrent à la recherche des mollusques, de ne point négliger l'étnde des animaux qui 
se rapportent aux différens groupes et aux deux sections de M. de Blainville, afin de pouvoir, par la suite, faire marcher avec les caractères de ces groupes, ceux des animaux qui leur appartiennent; ce qui nous fait insister à ce sujet, c'est que ce savant naturaliste siguale déjà des différences parmi quelques uns des animaux des Vénus; par exemple dans celui de la V. flexueuse, les tubes sont séparés, tandis qu'ils sont, en général, réunis. Dans quelques espèces, le pied se montre sémi-lunaire et sans sillon, tandis qu'ordinairement, il est triangulaire, tranchant et silloné inférieurement.

c. Coquilles irrégulières; toutes de mer.

G. CGLXXVI. VÉnénupe, venerupis, Lam.

Anim. oblong, assez épais, ayant les bords du manteau simples, un peu ouverts en avant pour le passage d'un pied comprimé et allongé; deux tubes assez longs, en grande partie réunis, et ayant leurs orifices radiés; branchies petites et faiblement inégales; appendices labiaux très petits.

Coq. solide, striée ou rayonnée, un peu allongẻe, bâillante en arrière, plus ou moins irrégulière, équilatérale, très inéquivalve, le côté antérieur étant toujours plus court que le côté postérieur; celui-ci géréralement comme tronqué, l'autre plus ou moins arrondi; sommets plus marqués, presque contigus; charnière composée de dents cardinales, grêles, rapprochées, et presque parallèles, au nombre de deux sur la valve droite, et trois sur la valve gauche, ou de trois dents sur chaque valve; ligament postérieur un peu allongé, et en grandé partie extérieur; impressions musculaires ovales, la postérieure plus arrondie, toutes deux étant réunies par une impression palléale très excavée en arrière.

Les coquilles qui composent ce genre sont lithophages; elles creusent dans les pierres et les madrépores des cavités plus ou moins proportionnées à leur forme et à leur 
volume où elles se logeut, et d'où elles ne peuvent plus sortir lorsqu'elles sont adultes, l'ouverture se trouvant alors trop petite. Elles sont sans épiderme et généralement d'un blanc sale. Il y en a de fossiles.

\section{G. CCLXXVII. Pétricole, petricola, Lam.} Et Rupellaire, Fleuriau de Bellevue.

Anim. ovale, épais, surtout à la partie supérieure; manteau à bords simples, un peu dilatés en avant, où ils forment une ouverture assez petite pour le passage d'un pied linguiforme et faible; tubes petits, en cônes tronqués à leur sommet, séparés dans les deux tiers de leur longueur, et finement radiés à leur orifice; branchies petites.

Coq. assez mince, non épidermée, blanche, rayonnée, ovale, subtrigone, bâillante en arrière, plus ou moins irrégulière, équivalve, inéquilatérale, le côté antérieur étant beaucoup plus court que le côté postérieur; sommets peu saillans, contigus; charnière composée de petites dents cardinales, peu divergentes, dont une au moins est bifide, au nombre de deux sur une valve, et une sur l'autre, ou de deux sur chaque; ligament extérieur, postérieur, court et bombé; impressions musculaires ovales, réunies par une impression palléale souvent faiblement distincte, ayant une excavation très profonde et arrondie en arrière.

Les Pétricoles ont les mêmes habitudes que les Vénérupes, et on en trouve souvent dans le même rocher, voisines les unes des autres. Kous en avons de fossiles.

G. CCLXXVIII. Coralliophage, coralliophaga, Blainv.

Cypricarde, Lam.

Anim. inconnu.

Coq. ovale, allongée, finement radiée du sommet à la base, cylindrique, équivalve, très inéquilatérale; 
sommets dorsaux très antérieurs et peu marqués; charnière subsimilaire; deux petites dents cardinales, dont une est subbifide, au-devant d'une sorte de dent lamelleuse, sous un ligarnent extérieur assez faible; deux impressions musculaires, petites, arrondies, distantes, réunies par une impresion palléale étroite, et assez excavée en arrière.

M. de Blainville a établi ce genre pour des coquilles placées par M. de Lamarck dans les Cypricardes, mais qui s'en distinguent bien, puisque, d'une part, l'excavation de l'impression musculaire annonce que l'animal a des tubes, et que de l'autre il est perforant. C'est particulièrement dans les masses de madrépores, si communes aux Antilles, qu'il faut les chercher. Le C. carditoïde est le type de ce genre.

\section{G. GCLXXIX. Cloтнo, clotho, Faujas.}

Anim. inconnu.

Coq. ovale, subrégulière, striée longitudinalement, équivalve, subéquilatérale ; charnière formée par une dent bifide, recourbée en crochet, un pen plus grande sur une valve que sur l'autre; ligament externe.

Ce genre ne comprend qu'une espèce fossile, la $C$. faubjasii, trouvée dans des coquilles de Cypricardes.

\section{G. CCLXXX. Onguline, ungulina, Daudin.}

Anim. inconnu.

Coq. longitudinale ou transverse, irrégulière, non bâillante, équivalve, subéquilatérale; sommets assez marqués et écorchés; charnière formée par une dent cardinale, courte et subbifide sur chaque valve, et d'une fossette oblongue, marginale, divisée en deux par un étranglement; ligament subintérieur, s'insérant dans ces fossettes; impressions musculaires allongées; impression palléale non flexueuse. 
Ce genre, encore peu connu, ne renferme que deux espèces vivantes dont la patrie est inconnue.

IO $O^{\circ}$ FAMTILE.

LES PYLORIDÉS, BlainV.

Anim. comprimé, de plus en plus cylindrique; le manteau de plus en plus fermé et prolongé en arrière par deux longs tubes ordinairement distincts, avec une ouverture antérieure et inférieure pour le passage d'un pied fort petit, et généralement conique; branchies étroites, libres et prolongées dans le tube.

Coq. régulière, rarement irrégulière, presque toujours equivalve, bâillante aux deux extrémités; charnière incomplète; les dents s'effaçant peu à peu; ligament interne ou externe; deux impressions musculaires distinctes, réunies par une impression palléale très flexueuse en arrière.

Tels sont les caractères de la famille des Pyloridés que nous empruntons à $M$. de Blainville. On voit que, par l'animal, elle diffère, en génèral, peu de la précédente, et qu'à la rigueur elle pourrait lui être réunie; cependant certains genres, les Solens, par exemple, présentent quelques caractères distinctifs, et nous pensons que lorsque l'on connaîtra mieux les animaux de la plupart d'entre eux, il couviendra peut-être de rétablir la famille des Solénacés, et peut-être aussi de porter quelques uns des genres de Pyloridés dans les Conchacés.

Nous avons provisoirement adopté cette famille et sa composition, à l'exception des Gastrochênes, des Arrosoirs et des Clavagelles qui ne sauraient y entrer, parce que, telle qu'elle est, elle indique assez bien la tendance à disparaître du système d'engrenage qui, dans la famille suivante, disparaitra totalement.

Les animaux de cette famille vivent tous dans le sable, la vase ou même dans les pierres; ils s'y enfoncent la partie antérieure la première, et s'élèvent ou s'abaissent à volonté au moyen de leur pied. Leurs coquilles sont généralement 
blanches, et rarement ornées de stries, autres que celles d'accroissement.

† Ligament interne.

a Coquille inéquivalve.

\section{G. CGLXXXI. Соnвule, corbula, Brug. Et Sphène, Turton.}

Anim. inconnu.

Coq. assez solide, subtrigone, bombée, faiblement irrégulière, trés peu bâillante, inéquivalve, inéquilatérale, arrondie en avant, atténuée et prolongée en arrière ; sommets fort distincts; charnière composée sur chaque valve d'une dent cardinale, conique, recourbée, correspondant avec une fossette qui lui est opposée; ligament intérieur et fort petit; impressions musculaires peu distantes; impression palléale faiblement excavée.

Ce genre renferme un petit nombre d'espèces vivantes et quelques unes fossiles. Les premières sont en partie des mers Australes et de celle de l'Inde; cependant il en existe une belle espèce dans l'Amérique du Sud, vivant dans l'eau saumâtre, et qui a été recueillie en grande abondance par M. Dorbigny, une petite espèce à la Martinique très connue sur les rivages, et une sur les côtes d'Angleterre, qui probablement habite aussi les nôtres, puisqu'elle a été retrouvée en Corse par M. Peyraudèau, c'est la $C$. nucleus. Turton a ćtabli pour la $C$. rostrata, le genre Sphène adopté par M. de Blainville, mais qui, d'après l'aveu de ce naturaliste, est à peine distinct des Corbules; nous ne croyons pas, d'après cela, devoir en surcharger encore cette famille. Attendons la connaissance de son animal pour lui imposer une distinction générique si elle le mérite.

G. CCLXXXII. Pandore, pandora, Brug.

Anim. ovalé, comprimé, assez allongé, ayant le manteau en forme de fourreau, se terminant en arrière par deux tubes réunis à leur base seulement, et assez courts, ouverts en avant pour le passage du 
pied, qui est grand, triangulaire, épais et renflé ẩ son extrémité; branchies grandes, libres en arrière, où les deux paires sont réunies, et se terminant en pointe dans le siphon; appendices labiaux assez grands, triangulaires et non striés.

Coq. mince, régulière, allongée, comprimée, inéquivalve, inéquilatérale, ayant la valve droite aplatie, et celle de gauche plus ou moins bombée; sommets peu distincts; charnière composée d'une dent cardinale sur la valve droite, correspondant à une cavité de celle de gauche; ligament interne, oblique, triangulaire, inséré dans une fossette à bords un peu saillans; impressions musculaires arrondies; celle du manteau peu apparente, et formant une petite excavation.

Deux espèces vivantes de nos côtes, ainsi que deux espèces fossiles, composent jusqu'à présent ce genre. Les Pandores vivent dans le sable, où elles s'enfoncent assez profondément pour que l'on ait beaucoup de peine à les en retirer.

G. CCLXXXIII. Thracie, thracia, Leach.

Anim. inconnu.

Coq. mince, bombée, ovale, peu allongée, inéquivalve; valve droite plus bombée que la gauche, inéquilatérale, à sommets bien marqués, un peu recourbés en avant; charnière dissemblable; une échancrure anguleuse un peu profonde, et en avant. une callosité nymphale étroite pour un ligament externe, sur la valve droite, correspondant à un cuilleron ou avance plus prononcé, et deux plis obliques de la valve gauche; deux impressions musculaires, petites, distantes, l'antérieure très abaissée et réunie à la postérieure par une ligule palléale assez rentrée en arrière.

Nous avons emprunté la caractéristique à $M$. de Blainville. 
G. CCLXXXIV. Périplome, periploma, Schumacher. Ostéodesme, Deshayes; Rupicole, Fl. de Bellev.

Anim. ovale, ayant le manteau épais et bilabié inférieurement, fermé partout, si ce n'est dans son tiers antérieur et inférieur, et pourvu en arrière d'un tube unique assez court; masse abdominale, petite, terminée par un pied lamelleux, tranchant et subantérieur; bouche petite, subovale, transverse, avec deux paires d'appendices larges et foliacés; branchies ovales, assez grandes, à stries très obliques d'arrière en avant, épaisses, unilobées de chaque côté, et complétement libres entre elles.

Coq. fort mince, fragile, ovale-oblongue, saillante, équi ou inéquivalve (la valve gauche plus bombée que la droite ), inéquilatérale; sommets peu marqués, comme fendus; charnière sans dents, ligament rouble, l'externe très mince, l'interne épais, porté sur des callosités nymphales assez saillantes, quelquefois en forme de cuilleron, et soutenu à son bord antérieur par un osselet transverse, hien symétrique; deux impressions distantes; impression palléale échancrée en arrière.

Nous empruntons encore à $M$, de Blainville la caractéristique de ce genre, qui, après avoir été établi par Schumacher, sous le nom de Périplome, l'a été par M. Deshayes sous celui d'Ostéodesme; nous avons dû rappeler cette première dénomination. L'espèce qui forme le type de ce genre, d'après M. de Blainville, est l'Anatina myalis, sur laquelle ce savant dit avoir pris les caractères que nous venons de voir. Cependant nous avons étudié tout récemment ce mêze mollusque que nous devons à l'obligeance de notre ami M. Deschères, et nous l'avons trouvé semblable à celui des Anatines, à l'exception que les deux tubes, au lieu de n'être séparés que dans une partic de leur longueur, le sont dans presque toute, caractère qui établit une grande différence entre l'animal de l'Anatince myalis et la caractéristique du genre Périplome, selon 
M. de Blainville. Nous pensons donc que ce n'est point l'A. myalis qui a servi aux observations de ce savant, mais peut-être quelque autre espèce.

b. Coquille équivalve.

\section{G. CCLXXXV. Andrine, anatina, Lam.}

Anim. oblong, allongé, assez épais, ayant le manteau fermé par une lame membraneuse assez large, avec une petite ouverture arrondie a la partie antéro-inférieure pour le passage d'un pied linguiforme; deux tubes en grande partie séparés à leur extrémité, allongés, l'inférieur l'étant un peu plus que le supérieur; branchies étroites, libres et pointues en arrière.

Coq. un peu mince, quelquefois translucide, ovale, allongée ou oblongue, bâillante à une ou deux extrémités, équivalve, très inéquilatérale; sommets en arrière, le bord supérieur antérieur étant plus long que le postérieur; charnière sans dents, mais ayant à la place une apophyse ou cuilleron horizontal, excavé, soutenu par une lame oblique et décurrente dans l'intérieur de la coquille, recevant le ligament; celui-ci interne; impressions musculaires distantes, ovales, réunies par une impression palléale peu wisarquee, ayant en arrière une excavation profonde et arrondie.

Ce genre ne contient qu'un petit nombre d'espèces toutes vivantes. L'une d'elles, qui est des mers de l'Inde, est une jolie coquille assez rare.

\section{G. CCLXXXVI. Mye, mya, Lam.}

Anim. oblong, un peu comprimé, enveloppé d'un manteau assez mince, adhérent par ses bords, fermé par une lame membraneuse, et formant en arriere, autour des tubes, une enveloppe lâche dans laquelle ceux-ci vienuent se retirer; tubes rcunis, de lon- 
gueur moyenne, un peu séparés à leur sommet et radiés à leurs orifices; pied très petit, conique, sortant du manteau par une petite fente située à sa partie antéro-inférieure, dans la ligne médiane; branchies moyennes, peu allongées, inégales du même côté; houche petite, ayant des appendices triangulaires striés comme les branchies.

Coq. enveloppée d'un épiderme se prolongeant sur les tubes et le manteau de l'animal; assez solide, bâillante aux deux extrémités, équivalve; sommets peu saillans; charnière composée d'un ou deux plis obliques, divergens en arrière d'un cuilleron horizontal, comprimé, appartenant à la valve gauche, et correspondant à une fossette également horizontale de la valve droitc; ligament interne s'insérant entre la fosselte et le cuilleron; impressions musculaires distantes, l'antérieure allongée, la postérieure arrondie; impression palléale étroite et profondément excavée.

Nous avons caractérisé l'animal sur la $M$. arenaria très commune sur nos côtes, où elle s'enfonce dans le sable; trois ou quatre autres espèces vivantes, et un plus grand nombre à l'état fossile, complètent ce genre.

\section{G. CCLXXXVII. Lutraire, lutraria, Lam.} Et Ligule, Leach.

Anim. ovale, allongé, comprimé, ayant le manteau assez largement ouvert en avant, et muni en arrière de deux tubes longs réunis ou séparés; pied asse 7. petit.

Coq. généralement assez allongée , quelquefois un peu arrondie, hâillante à chaque extrémité, régulière, équivalve, inéquilatérale, ayant les bords tranchans; sommets peu prononcés; charnière composée de deux petites dents cardinales divergentes, quelquefois peu sensibles, en avant d'une large fossette; deux ligamens, l'un interne, épais, inséré 
dans les fossettes, l'autre externe, postérieur et petit; impressions musculaires bien évidentes, l'antérieure ovale, allongée et courbée, la postérieure subarrondie; impression palléale étroite, ayant une excavation très profonde.

Ce genre renferme plusieurs espèces vivantes, appartenaut plutôt à nos mers qu'à celles des climats chauds, et une ou deux à l'état fossile.

†† Ligament externe et bombé.

G. CCLXXXVIII. Psammocole, psammocola, Blainv. Psammobie et Psammotée, Lam.

Anim. inconnu.

Coq. ovale, allongée, régulière, peu bâillante, équivalve, subinéquilatérale; sommets bien indiqués et un peu inclinés en avant; un angle souvent peu marqué sur le côté postérieur, ou le plus long; charnière à engrenage assez incomplet; une ou deux petites dents cardinales sur chaque valve; ligament extérieur très bombé; deux impressions musculaires bien distinctes, réunies par une impression palléale étroite, profondément excavée en arrière, et prolongée assez fortement au-delà.

Nous empruntons cette caractéristique à $\mathbf{M}$. de Blainville qui a, avec raison, établi le genre Psammocole pour y renfermer toutes les coquilles dont M. de Lamarck formait les genres Psammobie et Psammotée.

Voici la division que ce savant propose parmi les vingtsix espèces que l'on connait.

$$
\mathrm{r}^{\mathrm{er}} \text { groupe. Capsoides. }
$$

Coq. à peine bâillante, striée du sommet à la base, avec deux dents intrantes, obliques, divergentes sur chaque valve, mais plus grosses à gauche.

P. rugosa, ete. 
$2^{\mathrm{e}}$ groupe. Psammobies.

Cơ. plus bâillante, striée longitudinalement; les dents de la charnière beaucoup plus effacées.

$P$. virgata, etc.

$3^{\mathrm{e}}$ groupe. Psammotées.

Coq. de même forme; une seule dent cardinale sur chaque valve ou sur une seule.

$P$. violacea, etc.

Les Psammocoles sont de toutes les mers, et habitent les fonds sablonneux.

G. CCLXXXIX. Solételurn x, soletellina, Blainv.

Anim. inconnu.

Coq. ovale, allongée, comprimée, à bords minces et tranchans, équivalve, subéquilatérale, arrondie en avant, atténuée en arrière, où elle est subcarénée; sommets petits, mais assez prononcés; charnière composée d'une ou de deux petites dents cardinales; ligament épais, bombé, et porté sur des callosités nymphales assez relevées; deux impressions musculaires arrondies, surtout la postérieure ; impression palléale bien marquée, profondément et largement excavée en arrière.

Ce genre, qui ne renferme que quatre ou cinq espèces démembrées des Solens de M. de Lamarck, ne diffère que fort peu des Psammocoles au rapport de M. de Blainville.

G. CCXC. Sangurnolaire, sanguinolaria, Lam.

Anim. inconnu.

Coq. allongèe, subelliptique, très comprimée, faiblement bâillante à ses extrémités, à bord inférieur arqué, à bord postérieur non caréné; sommets petits et faiblement portés en avant; charnière composée sur chaque valve de deux dents cardi- 
nales convergentes au sommet et rapprochées; ligament saillant et bombé; impressions musculaires arrondies, réunies par une impression palléale étroite, profondément et largement excavée en arrière.

Ce genre ne renferme qu'un très petit nombre d'espèces toutes exotiques.

G. CCXCI. Solécurte, solecurtus, Blainv.

Anim, inconnu, mais probablement semblable à celui des Solens.

Coq. assez mince, demi-transparente, épidermée sur ses bords, ovale, très allongée, comprimée, équivalve, subéquilatérale, à bords presque droits et parallèles; extrémités également arrondies et comme tronquées; sommets extrêmement petits et non saillans; charnière composée de deux dents cardinales comprimées sur la valve droite, et de trois sur la valve gauche; ligament peu allongé, saillant, bombé, triangulaire; quelquefois une callosité oblique, décurrente à l'intérieur de chaque valve, sous le sommet; impressions musculaires assez, distantes, la postérieure subtriangulaire, l'antérieure oblongue, allongée; impression palléale étroite, dépassant de beaucoup l'impression musculaire antérieure, et munie en arrière d'une excavation.

Ce genre est établi aux dépens des Solens de M. de Lamarck; il renferme une dizaine d'espèces répandues dans différentes mers. Il y en a de fossiles.

\section{G. CCXCII. Solen, solen, Lam.}

Anim. très allongé, plus ou moins cylindrique ou comprimé; manteau fermé dans toute sa longueur, adhérent par ses bords, et lié au bord inférieur de la coquille par une double membrane qui se replie sur elle pour former l'épirlerme; jrésentant en ar- 
rière un seul tube double à l'intérieur, conique, annelé, susceptible de beaucoup d'allongement, avec les deux orifices simples, celui du siphon plus grand que celui de l'anus; ouvert tout-à-fait en avant pour le passage d'un pied gros, conique, renflé dans son milieu, pointu à son extrémité, qui termine en ligne droite le corps de l'animal; branchies longues, étroites, pointues en arrière, presque d'égale grandeur d'un même côté, adhérentes sur deux lignes, en avant, une de chaque côté du corps, se réunissant ensuite à une certaine distance en arrière, sur une seule, et alors libres et flottantes jusqu'à l'entrée du siphon; appendices labiaux non striés comme les branchies, allongés, triangulaires, recourbés, et dirigeant leur pointe en arrière; bouche petite; anus à l'extrémité d'un très petit tube flottant dans la cavité, au-dessus de la partie libre des branchies.

Coq. assez mince généralement, translucide, équivalve, extrêmement inéquilatérale, allongée, bâillante, tronquée aux deux extrémités, et à bords presque parallèles; sommets tout-à-fait antérieurs, à peine distincts; charnière composée d'une ou deux dents; ligament bombé, un peu allongé; inıpressions musculaires très distantes, l'antérieure oblongue, allongée, assez étroite, la postérieure subarrondie; impression palléale droite, fort lon-gue, terminée en arrière par une courte bifurcation.

Ce genre, qui ne comprend plus que les deux premières sections du genre Solen de M. de Lamarck, renferme une dizaine d'espèces vivantes. On en compte aussi quelques unes à l'état fossile.

\section{G. CCXCIII. Solémye, solemya, Lam.}

Anim. inconnu.

Coq. très mince, fragile, comprimée, allongée, axrondie à ses extrémités, à bords droits et parallèles, 
un peu bàillante en arrière, régulière, équivalve, très inéquilatérale, revêtue d'un épiderme luisant qui la ferme de toutes parts, excepté en avant et en arrière; côté antérieur beaucoup plus long que le postérieur; sommets peu distincts; charnière composée d'une dent cardinale, dilatée, comprimée, très oblique, un peu recourbée en dessus, servant à l'insertion du ligament sur chaque valve; ligament presque tout-à-fait postérieur; impressions musculaires petites, arrondies, écartées; quelques traces d'une impression palléale.

Les Solémyes, très voisines, saus doute, des Solens, mais dont elles diffèrent cependant assez par la position de leur ligament, sont des coquilles peu communes, parmi lesquelles on ne distingue que deux espèces, l'une assez grande qui appartient à la Nouvelle-Hollandc, et l'autre plus petite que nous avons trouvée quelquefois sur les rivages de Cette, c'est la $S$. mediterranea.

\section{G. CCXCIV. Glyamìne, glycimeris, Lam. Cyrtodère, Daudin.}

Anim. oblong, subcylindrique, ayant le manteau épais et ridé, fermé dans toute sa longueur, et ouvert tout en avant pour le passage d'un pied épais et tranchant; un seul tube en arrière, double à l'intérieur, court, très renflé et arrondi à son extrémité; lames brianchiales inégales d'un même côté, et assez grandes; appendices buccaux longs et en forme de demi-croissant, la pointe étant dirigée en arrière.

Coq. fortement épidermée, allongée, arrondie, bâillante aux deux extrémités, un peu irrégulière, équivalve, très inéquilatérale; sommets peu saillans écorchés; charnière sans dent, présentant seulement une callosité épaisse et un peu allongée; ligament extérieur porté par des nymphes saillantes, au-dehors; impressions musculaires distinctes et très écartées; impression palléale bien marquée, non 
excavée postérieurement; valves épaissies par des callosités à l'intérieur.

On ne connait que deux espèces vivantes appartenant à ce genre, et aucune des deux n'est fluviatile, comme quelques naturalistes en ont eu l'idée; elles proviennent des mers đe Terre-Neuve, où l'une d'elles, la G. siliqua, y est très commune; les pêcheurs du grand banc la trouvent. souvent dans le ventre des morues. M. Audouin, à qui nous devons les caractères de l'animal, a fait sur ce genre un travail anatomique accompagné de planches admirablement dessinées.

G. CCXCV. Panopíe, panopcea, Ménard de la Groie. Anim. inconnu.

Coq. épaisse, solide, bombée, oblique, allongée, bâillante aux deux extrémités, équivalve, inéquilatérale, un peu irrégulière; sommets bien marqués, contigus, un peu courbés en avant, et plus. rapprochés de la partie antérieure que de la postérieure; charnière présentant sur chaque valve une dent conique en avant d'une fossette, et d'une callosité épaisse, non saillante au-dehors, peu allongée, sur laquelle s'insère le ligament; ligament extérieur, postérieur et bombé; impressions musculaires irrégulièrement ovales, l'antérieure étant la plus longue, toutes deux réunies par une impression palléale, large, parailèle au bord de la coquille, et assez profondément excavée en arrière.

Ce genre est formé pour une seule espèce vivante, la $\boldsymbol{P}$. aldrovandi, grande et belle coquille très rare dans les collections avec les deux valves, et que M. de Lamarck prétend appartenir à la Méditerranée, mais que nous u'avons jamais pu y découvrir. Elle paraît habiter les fonds vaseux. On en connait aussi des espèces fossiles.

G. CCXCVI. Saxicave, saxicava, Fl. de Bell.

Anim. allongé, subcylindrique, ayant le manteau fermé de toutes parts, prolongé en arrière par un 
tube long, double à l'intérieur, un peu divisé à son sommet, et percé inférieurement et en avant d'un orifice arrondi pour le passage d'un petit pied allongé, mince et pointu ; bouche moyenne; appendices labiaux petits; lames branchiales en grande partie libres et très inégales d'un même côté. Coq. épaisse, solide, épidermée, allongée, arrondie en avant, comme tronquée en arrière, bâillante, irrégulière, équivalve, très inéquilatérale, le côté postérieur étant beaucoup plus long que l'antérieur; sommets peu distincts; charnière sans dents ou avec deux tubérosités écartées plus ou moins prononcées; ligament extérieur; impressions musculaires arrondies et un peu rapprochées, réunies par une petite impression palléale droite, très étroite, occupant le milieu de la valve.

Nous avons caractérisé l'animal de ce genre sur la Saxicava rugosa qui est très commune à La Rochelle, et notre description ne diffère que très peu de celle donnée par M. de Blainville d'après la $S$. australis. Quatre ou cinq espèces toutes vivantes et lithophages composent ce genre.

\section{G. CCXCVII. Byssome, byssomia, Cuv.}

Anim. plus ou moins long, subcylindrique, ayant le manteau fermé, mais percé d'une petite ouverture à la partie antérieure et inférieure pour le passage d'un pied pelit, conique, canaliculé, muni d'un byssus à sa base postérieure; un long tube double, bifurqué à son extrémité.

Coq. épidermée, oblongue, irrégulière, souvent grossièrement striée en long, équivalve, très inéquilatérale, très haute en avant, et atténuée en arrière; sommets peu marqués, cependant distincts, faiblement courbés en avant; charnière sans dents, ou ne présentant qu'un rudiment de dents sous le corselet; ligament extérieur allongé; impressions musculaires fortes, distantes et arrondies. 
Ce genre, très voisin des Saxicaves, en diffère cependant suffisamment par la présence d'un byssus; les Byssomies se logent dans les petites cavités des rochers, ou même des plantes marines; on en trouve encore quelquefois dans le sable.

G. CCXCVIII. Rномвоїрғ, rhomboïdes, Blainv.

Anim. rhomboïdal, allongé, assez comprimé; deux tubes distincts en arrière; une fente assez large à la partie antérieure et inférieure du nanteau, pour la sortie d'un pied conique et d'un byssus dont les filets sont élargis à l'extrémité.

Coq. rhomboïdale, un peu irrégulière, striée en longueur, équivalve, très inéquilatérale; sommets très distincts et très antéro-dor'saux; charnière fermée par deux petites dents cardinales; ligament externe, postérieur, assez saillant ; deux impressions musculaires arrondies.

Ce genre, dont nous copions textuellement la caractéristique dans le Manuel de Malacologie, est établi par M. de Blainville pour le Mytilus rugosus de Gmelin. II nous paraît extrêmement rapproché des Byssomies.

\section{G. CCXCIX. Hiatelle, hiatella, Daudin.} Biapholius, Leach.

Anim. inconnu.

Coq. mince, allongée, rhomboïdale, équivalve, très inéquilatérale, bâillante à son bord inférieur ainsi qu'en arrière ; sommets antérieurs, et recourbés en avant; charnière composée d'une dent sur une valve correspondant à une fossette de la valve opposée, ou bien d'une petite dent avec une fossette sur chaque valve; ligament extérieur.

Ce genre fort incomplétement connu, et dont la place n'est peut-être point ici, contient trois espèces exotiques que nous ne connaissons pas. 
I I FAMILLE.

\section{LES TUBICOLÉS, Lam.}

Et Pholadaires, Lam.; Enfermés, Cuv.; Tubicolés et Pholades, Fér.; Pyloridés et Adémavés, Blainv.; Solénides, Pholadaires et Térédinites, Lat.

Anim. sphérique, allongé ou vermiforme, ayant le manteau fermé et plus ou moins tubuleux, avec une petite ouverture antérieure pour le passage d'un petit pied; deux tubes généralement assez allongés, réunis en un seul, et servant, l'inférieur, à la respiration, et le supérieur, aux déjections; les appendices labiaux petits; les branchies allongées, se prolongeant jusque dans le siphon.

Coq. généralement non épidermée, toujours blanche, plus on moins allongée, rarement sphérique, quelquefois comme tronquée, équivalve, inéquilatérale, bâillante à ses deux extrémités, surtout en avant; charnière sans engrenage bien caractérisé; ligament rarement évident, et quelquefois remplacé par des appendices du manteau de l'animal; les impressions musculaires plus ou moins distinctes, la postérieure l'étant toujours davantage, toutes deux réunies par une impression palléale plus ou moins sensible, et toujours fortement excavée en arrière.

Un tube calcaire, soit libre on soudé, enveloppant ou non, et se prolongeant en arrière, ou au moins des pièces accessoires pour clore la coquille d'une manière plus complète.

Marins, se logeant dans les pierres, où ils creusent des cavités dont ils ne peuvent plus sortir, ou s'enfonçant dans la vase et le sable.

† Point de cuilieron à l'intérieur; encore quelques vestiges de charnière; les impressions musculaires visibles.

a) Un tube soudé avcc les deux valves. 
G. CCC. Anrosorr, aspergillum, Lam.

Anim. inconnu.

Coq. subnacrée, équivalve, subéquilatérale, cuverte et fixée par ses contours dans la paroi d'un tube; sommets très distincts et un peu recourbés en avant; charnière et impressions musculaires inconnues; ligament effacé.

Tube calcaire, épais, solide, très allongé, plus on moins régulièrement droit, conique ou subcylindrique, quelquefois un peu comprimé et faiblement cannelé latéralement; ouverture terminale, postérieure, oblongue ou arrondie, simple ou bordée d'une ou plusieurs rangées de plis semblables à des manchettes; extrémité antérieure, fermée par un disque convexe percé d'un nombre considérable de trous arrondis, plus ou moins tubuleux, avec une fissure au milieu.

On ne connaît point encore l'animal de l'Arrosoir; cependant on ne peut plus douter aujourd'hui que ce ne soit un mollusque; la connaissance assez complète que nous avons de la Clavagelle nous apprend que ces deux genres sont tellement voisins, se lient à un si haut degré par leurs plus importans caractères, qu'il couviendrait peutêtre de les réunir; la seule différence que l'on puisse maintenant établir entre eux, c'est que l'uve de ces coquilles a ses deux valves soudées dans la paroi da tube, tandis que l'autre n'en a qu'une seule de soudée, l'autre étant entièrement libre; ce caractère est-il bien propre à former une distinction générique? Nous concevons l'animal de l'Arrosoir, absolument comme celui de la Clavagelle, et nous pensons, avec M. de Blainville, que ces deux mollusques sont munis d'un byssus, qu'ils font sortir par tontes les ouvertures antérieures du tube pour se fixer sur les corps; il convient sans doute aussi de leur attribuer les mêmes habitudes, celle, par exemple, de se loger dans les corps; mais comme il n'est pas probable que l'animal de l'Arrosoir puisse, comme cclui de la Clavagclle, se servir 
dans le jeune âge de ses petites valves pour se creuser une cavité, il y a lieu de croire que c'est uniquement dans le sable ou la vase qu'il s'enfonce, le disque le premier, et la partie tubuleuse en haut. Deux motifs se joignent encore à celui-ci pour appuyer cette idée; d'abord cette quantité de sable ou de débris de coquille, quelquefois très grande, surtout dans une espèce, qui vient se fixer à la surface extérieure du tube; ensuite la forme allongée, et toujours assez droite, de celui-ci, qui indique que l'animal en le formant, et l'élevant à la surface du sol, ne rencontrait aucun obstacle; dans certaines Clavagelles, au contraire, la flexuositè plus ou moins grande du tube annonce, comme dans ceux des Tarets et des Cloisonnaires, que l'animal vivait dans des corps durs.

Nous pensons que l'animal de l'Arrosoir s'enfonce dans le sable pendant qu'il est jeune, qu'il s'y fixe par le moyen de son byssus aux corps qu'il rencontre, comme on le voit fréquemment dans certaines moules, et que ce n'est qu'ensuite qu'il forme son disque, et qu'il élève son tube vers la mer.

On ne connaît que quatre espèces de ce genre, toutes fort rares, et de l'hémisphère austral, et on peut les distinguer de la manière suivante :

$$
\mathrm{I}^{\mathrm{er}} \text { groupe. }
$$

Tube sans couronne antérieure.

A. agglutinans, etc.

$$
\mathbf{2}^{\mathrm{e}} \text { groupe. }
$$

Tube muni en avant d'une couronne, formée de petits tubes serrés les uns contre les autres.

A. javanus, etc.

M. Defrance signale aussi deux espèces fossiles

b) Un tube soudé avec une seule valve.

\section{G. CC.CI. Glavagelle, clavagella, Lam.}

Anim. inconnu.

Coq. ovale, plus longue que haute, peu épaisse, assez 
solide, quelquefois transparente, régulière, équivalve, inéquilatérale, bâillante; sommets très distincts, peu proéminens, plus ou moins antérieurs, rapprochés; charnière offrant toujours une petite dent plus ou moins fermée sur chaque valve; ligament mince, en partie interne, inséré dans une très petite fussette; impressions musculaires très distinctes, écartées, l'antérieure moins rapprochée du bord supérieur que la postérieure, toutes deux ovales et réunies par une ligule palléale bien marquée, et profondément excavée en arrière.

Tube calcaire, épais, solide, irrégulier, droit ou sinueux, subcylindrique, plus ou moins allongé, quelquefois comprimé et faiblement cannelé latéralement, avec une ouverture irrégulièrement circulaire ou oblongue en arrière, formant une massue en avant pour envelopper la coquille, dont une des valves, la valve gauche, est enchâssée, soudée dans la paroi, et visible à l'extérieur, tandis que l'autre est libre et cachée dans l'intérieur; massue terminée en avant par des ouvertures tubuleuses, irrégulières, et diversement disposées.

Nous caractérisons ce genre sur deux espèces très distinctes que nous arons en ce moment sous les yeux : l'une est la C. couronnée, incomplétement connue jusqu'à ce jour, et dont nous possédons un grand nombre d'individus plus ou moins parfaits; et l'autre, la C. râpe, senle espèce connue à l'état vivant, et que nous venons de découvrir à l'ile de Bourbon; nous avons aussi un très bon dessin, sur plusieurs faces, de la $\mathrm{C}$. à crête de $\mathrm{M}$. de Lamarck (nous ne connaissons aucune des autres espèces). La comparaison que nous faisons de ces trois coquilles, ne nous permet plus de penser que le genre Clavagelle soit artificiel, comme M. de Blainville parait disposé à le croire. Sans doute, sa coquille étant incomplétement connue, on ponvait lui trouver quelque analogie avec celles de certains autres genres; on prouvait même, frappé de la variété de forme qu'elle présente réellement dans les différentes es- 
pèces, les partager entre plusieurs genres des familles précédentes; mais maintenant nous nous croyons suffisamment fondé à repousser cette idée. En effet, la charnière des deux espèces, que nous examinons en ce moment, est la même; c'est toujours une petite dent sur chaque valve. Dans la disposition de leur ensemble, et dans celle de leur tube, nous trouvons les mêmes caractères; le mode d'accroissement de ce tube et son développement sont semblables, et enfin elles paraissent avoir toutes l'habitude de se loger dans les corps sous-marins pour y chercher un second abri.

Nous sommes donc fort loin de penser qu'elles puissent appartenir aux Vénus irrégulières, comme M. de Blainville parait en avoir l'idée, tant parce que les Clavagelles ne sont point irrégulières, que parce qu'elles n'offrent dans leur charnière aucun des caractères d'engrenage des Vénus.

Nous croyons le genre très naturel, et nous y rapportons, sans aucun doute, les trojs espèces que nous venons de citer; ne connaissant point les autres, nous ne pouvons rien affirmer à leur sujet. Nous ne comprenons même pas bien la $C$. tibiale; les deux valves y sont à découvert, dit-on, à la partie antérieure; sont-elles toutes deux soudées au tube, ou toutes deux libres? Dans les deux cas, nous la croyons hors du genre.

Voici l'observation que la C. râpe nous a donné lieu de faire; nous l'avons trouvée fort jeune dans un madrépore roulé; elle occupait une petite cavité dont l'ouverture trop étroite ne pouvait lui permettre de sortir. Un autre trou voisin de celui où était cette coquille, mais privé de son habitant, nous ayant paru semblable au premier, mais ouvert à l'extérieur par un col allongé, nous avons pensé qu'à un certain âge, la Clavagelle s'enveloppe d'un tube auquel elle n'adhère que par une de ses valves, et que ce tube, se prolongeant jusqu'en dehors de la cavité du madrépore, lui conserve un orifice assuré autour duquel les polypes peuvent s'élever et s'étendre sans lui faire courir le danger de voir sa demeure se fermer, car elle peut, par de nouveaux accroissemens, étendre ce tube à une graude distance. C'est précisément ce que l'on remarque chez quelques autres mollusques, et notamment chez la Magile qui, pour tenir saus cesse son orifice au niveau 
des masses madréporiques, que tant de petits polypes travaillent saus cesse à augmenter, est obligée d'allonger progressivement son tube.

Il y a lieu de penser aussi que certaines espèces de Clavagelles peuvent s'introduire dans les coquilles mêmes, car on en a trouvé dans l'intérieur des Crassatelles.

Quant à l'animal, nous ne le connaissons pas; mais l'analogie de ce tube avec celui des autres genres de cette famille, nous donne tout lieu de croire qu'il est muni en arrière de deux canaux tubuleux réunis; les petites ouvertures spiniformes, qui se montrent en avant de la massue, paraissent destinées au passage d'un byssus qui aurait pour but de fixer l'animal et sa coquille au fond de sa demeure; nous croyons même pouvoir affirmer que c'est uniquement là l'usage de ces petits tubes, non que nous ayons trouvé des débris de ce byssus au fond de la cavité, mais parce que nous avous cru y reconnaître quelques empreintes correspoudantes aux tubes.

Nous divisons ce genre en troís groupes; mais le dernier est douteux pour nous.

$$
\text { Ier groupe. }
$$

Coq. terminée antérieurement par un disque aplati, portant une fissure sur son centre.

C. coronata, etc.

Les espèces de ce ‘premier groupe sont très voisines des Arrosoirs.

$$
2^{e} \text { groupe. }
$$

Coq. terminée antérieurement par une crête.

C. crislata, etc.

$$
\text { 3e groupe, douteux. }
$$

Coq. ayant les deux valves à découvert; point de tube.

C. tibialis, etc. 
Il est probable que les autres espèces décrites par M. Deähayes se rapportent au second groupe.

On a découvert récemment dans les mers de Sicile une coquille assez voisine des Clavagelles, mais que, selou nous, on ne doit pas rapporter à ce genre. Cette coquille, logée dans une cavité particulière creusée dans les pierres, a ses valves libres, taudis que l'ouverture de cette cavité est nunie supérieurement d'un tube faisant l'effet d'une cheminée, bordé à son orifice d'une manchette analogue a celle de certains Arrosoirs. Cette coquille ne peut point appartenir aux Clavagelles qui ont une valve soudée ; nous pensons plutôt qu'elle devra former un genre dans le voisinage des Gastrochènes, car probablement le tube ne s'élève pas seulement à l'orifice de sa demeure. Il y a lieu de croire que, comme dans les Gastrochènes, il se prolonge inférieurement dans cette cavité qu'il tapisse, et par ce moyen enveloppe la coquille; ce serait donc avant les Gastrochènes, et dans la division $c$ ), qu'il faudrait la placer.

c) Quelquefois un tube enveloppant toute la coquille et non soudé.

G. CCCII. Gastrochìne, gastrochana, Spengler.

Anim. ovale, ayant le manteau fermé avec une très petite ouverture antérieure, arrondie, pour le passage d'un petit pied conique ou linguiforme; les tubes allongés et réunis dans toute leur longueur. Coq. mince, oblique, ovale, cunéiforme, équivalve, très inéquilatérale, extrêmement bâillante à sa partie antéro-inférieure; sommets assez marqués ; charnière sans dents, droite, linéaire; une apophyse se montrant souvent en dessous de la charnière, dans l'intérieur de chaque valve; ligament externe; impressions musculaires distinctes, liées par une impression palléale peu sensible et excavée postéricurement.

Quelquefois un tuhe calcaire, ampulliforme, court, 
à ouverture arrondie, enveloppant la coquille, et tapissant la cavité de la pierre.

Toutes les Gastrochènes n'ont point un tube calcaire, mais toutes s'enfoncent dans les pierres à la manière des Pholades; deux espèces qui appartiennent au genre Fistulane de M. de Lamarck, viennent maintenant se ranger dans celui-ci, et c'est à $\mathbf{M}$. de Blainville que l'on doit ce rapprochement; ce sont les $\mathrm{F}$. massue et ampullaire.

Les deux groupes suivans découlent naturellement de ce rapprochement.

$$
{ }^{\text {er }} \text { groupe. }
$$

Coq. lisse sans tube distinct.

G. cuneiformis, etc.

$$
2^{\mathrm{e}} \text { groupe. }
$$

Coq. striée du sommet à la base, contenue dans un tube distinct.

G. clava, etc.

M. Charles Des Moulins qui, depuis très long-temps, et avant tout ce qui a été dit à ce sujet, avait découvert l'existence d'un tube dans les Grastrochènes, nous a montré ce tube non seulement dans les espèces vivantes de nos côtes, mais encore dans celle fossile à Mérignac.

†† Un cuilleron ou une pièce sétiforme à l'intérieur; nulle vestige de charnière, les impressions musculaires de moins en moins visibles.

a) Des pièces accessoires.

I. Rarement un tube.

\section{G. CCCIII. Pholade, pholas, Lin.}

Anim. plus ou moins épais et allongé, rarement raccourci; manteau se réfléchissant à la partie dorsale pour lier ensemble les valves et les pièces accessoires; ouverture antérieure assez petite; pied court, oblong et aplati; tubes souvent allongés. 
et réunis en un seul très extensible et dilatable; bouche petite, avec de très petits appendices labiaux; branchies allongées, étroites, un peu inégales de chaque côté, réunies sur une même ligne dans presque toute leur longueur, et se prolongeant jusque dans le siphon.

Coq. mince, lactée, un peu transparente, recouverte quelquefois d'un faible épiderme; ovale, allongée, équivalve, inéquilatérale et bâillante en arrière, et surtout à la partie antéro-inférieure ; sommets cachés par une callosité; charnière sans dents; ligament douteux? un cuilleron aplati, recourbé, élargi à son extrémité, s'élevant dans chaque valve, en dedans du sommet; impressions musculaires très distantes, la postérieure grande, oblongue, allonģée, toujours très visible; l'antérieure petite, arrondie, peu distincte, toutes deux plus ou moins rapprochées du bord de la coquille, surtout l'antérieure, et liées par une impression palléale, longue, étroite, profondément excavée en arrière. Plusieurs pièces accessoires ou aucune? quelquefois un tube calcaire enveloppant de toutes parts, mais laissant une ouverture en arrière.

Si l'on connaissait d'une manière plus complète les espèces qui composent le genre Pholade, on pourrait les partager en plusieurs groupes très bien caractérisés par le nombre et la disposition des pièces accessoires qui varient considérablement; mais malheureusement on ne connait suffisamment ces pièces accessoires que dans un petit nombre d'espèces. Du reste, le genre en lui-même est si mal connu, quoique se trouvant en une singulière abondance sur nos côtes, que les naturalistes ne sont pas encore d'accord sur le nombre de leurs impressions musculaires. M. de Lamarck les comprend daus les Dymiaires, et M. de Blainville, toujours si soigneux observateur des caractères, ne voit qu'une seule impression. Quant à nous, nous ne doutons pas un seul instant que les Pholades n'aient deux 
impressious; nous l'avons positivement reconnu sur le pholas costata, en suivant l'impression palléale à partir de l'impression musculaire postérieure, qui est toujours assez évidente, jusqu'à l'endroit où la première se termine en avant. Là, on distingue très bien une petite impression irrégulièrement arrondie. Elle a été également observée par M. Charles Des Moulins sur la même espèce; mais la science doit à ce naturaliste une observation peut-être plus importante au sujet des Pholades, c'est que ces coquilles se montrent quelquefois accompagnées d'un tube calcaire, appliqué comme celui des Gastrochènes à la paroi interne de la cavité qu'elles habitent. Nous n'avons point encore pu vérifier ce fait avec lui sur des espèces vivantes; mais il nous en a montré plusieurs fossiles de Mérignac dans lesquels nous avons complétement reconnu ce caractère important qui établit davantage les rapports que l'on admettait entre les Pholades, les Tarets et les Fistulanes.

Il y a quelques espèces de Pholades qui semblent conduire aux Tarets. Ces coquilles habitent dans les pierres, les madrépores, le bois et quelquefois même dans la vase. Lorsque la mer, par son reflux, les laisse à découvert, et que leurs animaux viennent à être inquiétés, ils lancent par leur siphon, et à une assez grande distance, l'eau que contient leur manteau, et qui baigne les branchies.

G. CCCIV. Jounnnétıe, jouannetia, Ch. Des Moul.

Anim inconnu, mais ayant certainement les caractères de la famille.

Coq. sphérique, cunéiforme, équivalve, inéquilatérale, à peine bàillante en arrière, largement bâillante en avant, a valves solides, courtes, courbes, pointues inférieurement, striées obliquement, les stries convergentes vers un sillon médian; sommets peu distincts, étant recouverts de pièces accessoires soudées; un écusson très vaste, lisse, mince, fragile, enveloppant avec l'âge toute la partie antérieure, formé de deux moitiés un peu inégales, s'emboîtant l'une dans l'autre, soudées. chacune par un de leurs bords à l'une des valves, 
et resserrant de cette manière le bâillement antérieur de la coquille; point de ligament ni d'engrenage; un appendice sétiforme, vertical, procédant du sommet, soudé à l'intérieur de chaque valve, et occupant le tiers de sa hauteur; impressions musculaires encore inconnues; impression palléale très forte, et profondément excavée en arrière.

Des pièces accessoires, mais soudées; point de tube calcaire enveloppant, le vaste écusson en tenant lieu.

C'est une jolie découverte que celle de la Jouannétie, que M Charles Des Moulins a dédiée à si juste titre au savant modeste, à qui la science doit de si beaux travaux sur la géologie et les antiquités du département de la Gironde qu'il habite. Quand des dédicaces de ce genre seront justifiées par autant de titres, il n'est pas douteux qu'on ne s'empresse de les conserver comme un hommage justement mérité.

Ce genre, que nous avons étudié avec M. Des Moulins lui-même, est bien distinct de celui des Pholades, et sa. place est parfaitement fixée entre celles-ci et les Tarets auxquels il conduit si naturellement par ses valves. La Jouannétie ue montre pas de tube calcaire enveloppant comme il arrive quelquefois aux Pholades, etc., et quoique nous ne connaissious encore qu'une seule espèce, nous pensons qu'un pareil tube n'existe jamais, si toutefois l'ampleur et la disposition de l'écusson, qui nous paraît en tenir lieu, est un caractère générique. L'espèce connue est la $J$. semicaudata; elle est fossile des faluns libres de Mérignac, et se trouve dans l'intérieur des madrépores et des morceaux de. caicaire roulé.

b) Point de pièces accessoires.

2. Un tube enveloppant, nou soudé.

G. CCCV. Tanet, teredo, Linné.

Anim. très allongé, vermiforme, ayant le manteau, très mince, ouvert en avant et à sa partie infé- 
rieure, pour le passage d'un pied en forme de mamelon; tubes séparés, très courts, surtout celui des déjections; bouche petite; appendices labiaux courts; anus situé à l'extrémité d'un petit tube flottant dans la cavité du manteau; branchies rubanées, réunies sur une même ligne dans toute leur longueur, et un peu prolongées dans le siphon; un anneau musculaire au point de réunion du manteau et des tubes, dans lequel est implanté une paire d'appendices ou palmules cornéo-calcaires , pédiculés, jouant latéralement l'un vers l'autre.

Coq. assez épaisse, très courte, annulaire, également ouverte en avant comme en arrière; équivalve inéquilatérale, anguleuse, à valves triangulaires, tranchantes en avant, et ne se touchant que par les deux bords opposés; charnière nulle; un cuilleron allongé, presque droit, subfiliforme; une seule impression musculaire faiblement distincte, l'autre probable par analogie.

Tube cylindrique, droit ou flexueux, fermé avec l'âge à l'extrémité buccale, de manière à envelopper l'animal et sa coquille, toujours ouvert par l'autre, et tapissant la cavité dans laquelle l'animal s'est introduit.

Ce genre renferme plusieurs espèces plus ou moins connues; l'une d'elles est très commune sur nos côtes, dans nos ports, et même dans nos rivières où elle perce les bois de manière à les détruire promptement; c'est le $T$. navalis qui, dit-on, a été importé dans les mers d'Europe par les bâtimens venant des régions australes. Plusieurs espèces sont encore douteuses, parce que l'on ne connait le plus souvent que le tube et non la coquille et les palmules singulières, dont l'animal est muni. Il serait donc à désirer que les voyageurs s'occupassent particulièrement de la recherche de ces mollusques. Jusqu'à présent on n'a signalé qu'une seule impression musculaire à chaque valve des Tarets, encore est-elle faiblement distinete; nous pensons que, malgré cela, cette coquille appartient aux Dimyaires, 
car son analogie avec les Pholades est trop évidente pour qu'on ne lui suppose pas au moins les mêmes caractères de premier ordre. Il en est, saus doute, des Tarets comme des Pholades, chez qui nous venons, pour la première fois, de signaler cette seconde impression musculaire que l'on n'avait pas encore aperçue, et qui cependant est tellement manifeste dans certaines espèces qu'on ne peut conserver de doutes à son sujet; certainement les Tarets ont un second muscle, mais dont l'impression échappe, sans doute, à l'œil sur une coquille aussi bornée, et nous sommes assez porté à croire que c'est ce second muscle qui transmet à chaque valve le mouvement par lequel elles creusent le bois. Les 'Tarets sont extrêmement communs, et font de grands dégâts dans les ports. Tout le monde sait que la Hollande faillit être submergée par suite de ceux qu'ils causèrent dans les digues qui font toute sa sûreté; en s'introduisant dans le bois, ils suivent une direction tortueuse; cependant il est facile de s'apercevoir qu'en général ils s'avancent dans la direction des fibres ligneuses.

M. Defrance cite quelques espèces à l'état fossile.

\section{G. CCCVI. Fisturane, fistulana, Brug.}

Anim. semblable à celui des Tarets, mais plus court. Coq. épaisse, courte, annulaire, très ouverte antérieurement et postérieurement, équivalve, inéquilatérale, non tranchante en avant, sans charnière, mais munie d'un cuilleron allongé; impressions musculaires inconnues.

Un tube peu allongé, épais, solide, fermé en avant, de manière à envelopper la coquille, généralement atténué en arrière, où son ouverture laisse plus ou moins apercevoir une cloison qui sert à la séparation des tubes de l'animal; présentant quelquefois des cloisons en voûte, dans le fond de sa cavité.

Les Fistulanes péuètrent daus le sable et même daus le bois, comme nous avons eu occasion de nous en assurer, aiusi donc l'opinion de $M$. de Blainville est fondée, quand il dit que ce genre pourrait être supprimé, tant il est voisin des 'Tarets; aussi nous ve l'aurions pas reproduit d'après 
une telle autorité, si nous ne pensions qu'il convient mieux de laisser à quelques naturalistes plus éclairés que nous sur ces animaux, l'avantage de pouvoir, avec connaissance de cause, supprimer définitivement ce double emploi.

M. de Lamarck indique six espèces de Fistulanes; mais M. de Blainville en retire deux pour les porter dans le genre Gastrochène. L'une des quatre espèces qui restent est fossile.

Nous venons de trouver dans les terrains crayeux de Royan le moule d'un tube fossile qui appartenait, sans doute, à une espèce de ce genre, mais qui établit d'une manière assez remarquable le rapprochement des Fistulanes aux Tarets; car il indique un tube fermé à l'une de ses extrémités, et muni de six à sept cloisons voûtées, complètes, inégalement distantes, comme cela peut arriver dans les Fistulanes, et en même temps il est très allongé, irrégulièrement sinueux et de grosseur presque égale dans toute sa longueur, comme celui des Tarets.

\section{Cloisonnare, septaria, Lam.}

Anim. inconnu.

Coq. inconnue.

Tube calcaire, épais, solide, en cône très allongé, et irrégulièrement flexueux, muni intérieurement de petites cloisons annuliformes, incomplètes; terminé à une de ses extrémités par un renflement, et à l'autre par deux tubes grêles et séparés.

Tout porte à croire que ces singuliers tubes, qui sont quelquefois d'une grande dimension, et qui appartiennent bien certainement à un mollusque, sont très voisins de ceux des Tarets et des Fistulanes. Romphius semble confirmer cette opinion en parlant des deux osselets que porte l'animal.

Nous conserverons ce genre en attendant de plus amples renseignemens. Une seule espèce lui sert de type; elle vit dans les sables, et vient des mers de l'Inde. On en voit dans la collection du Jardin du Roi de maguifiques exemplaires, mais incomplets.

3. Un tube soudé. 
G. CCCVIII. TÉrédine, teredina, Lain.

Anim. inconnu.

Coq. épaisse, bombée, ovale, assez courte, très bâillante en arrière, équivalve, inéquilatérale, à sommets très distincts, un peu recourbés en avant, munie en dedans d'un cuilleron épạis sur chaque valve.

Tube calcaire, épais, solide, subcylindrique, à ouverture terminale postérieure, sans cloison, se réunissant á la partie postérieure des deux valves par une sorte d'écusson.

Ce genre ne contient que des espèces fossiles; il cst encore assez incomplétement connu.

$2^{e}$ SECTION. ACÉPHALES NON TESTACÉS, Cuv.

$$
\text { 3e ORDRE. }
$$

\section{HÉTÉROBRANCHES, Blainv.}

Anim. de forme assez variable, généralement un peu allongé, enveloppé de toutes parts d'un manteau dur, épais ou gélatineux, quelquefois transparent, et percé de deux ouvertures; bouche, anus et branchies à l'intérieur, celles-ci de formes diverses, mais jamais divisées en quatre feuillets, toujours renfermées dans la cavité intérieure.

Coq: nulle.

Marins.

I T PA MILLE.

\section{LES ASCIDIENS, Lam.}

Anim. de forme très variable, enveloppé d'un manteau épais, rugueux, souvent coriace, contractile; adhérent aux corps sous-marins par l'extrémité inférieure, terminé à sa partie opposée par deux 
ouvertures quelquefois tubuleuses, souvent garnies sur leurs bords de papilles tentaculaires, l'une, qui est toujours la plus élevée, recevant l'eau nécessaire aux branchies, et l'autre servant d'orifice au canal commun, à l'anus et à l'appareil générateur; bouche au fond de la cavité branchiale; branchies en forme de réseau tapissant cette cavité.

Cette famille, et particulièrement les premiers genres qui la composent, lient parfaitement les Hétérobranches avec les Lamellibranches, car on reconuait dans l'organisation des Ascidiens, à peu de chose près, la mêrne disposition que dans celle des animaux des deruières familles, que nous venons de décrire, quoiqu'au premier aspect il paraisse y avoir une grande différence. L'enveloppe des Ascidiens est double, c'est-à-dire qu'elle se compose d'abord d'une tunique intérieure assez mince, fibreuse et vasculaire, et ensuite d'un manteau plus ample, enveloppant, cartilagineux et épais.

Ces animaux se fixent sur les rochers et autres corps sous-marins, généralement à des profondeurs très grandes; ils sont de toutes les mers, et très nombreux, mais encore imparfaitement connus, du moins la plupart des genres.

$†$ Ascidiens simples.

\section{G. CCCIX. Ascidie, ascidia, Jin.}

Anim. ovoïde, plus ou moins allongé, quelquefois cylindrique, très variable dans sa forme, ayant le manteau cartilagineux plus ou moins épais et consistant, élargi ou pédiculé à sa base, et terminé supérieurement par deux tubes courts, inégaux, à orifices rayonnès par la présence de papilles tentaculaires.

Ce genre très nombreux, mais dont on ne connait encore qu'une trentaine d'espèces, pourrait être divisé en deux groupes de la manière suivante: 
rer groupe.

Animal à base élargie.

A. intestinalis, etc.

$2^{e}$ groupe.

Animal à base pédiculée.

A. clavala, etc.

Dans le nombre des espèces il y en a quelques unes remarquables par leurs couleurs vives; mais en général elles ne sont point d'un aspect agréable à l'œil. Nous avons souvent remarqué qu'elles pouvaient vivre assez long -temps hors de l'eau. Ces mollusques paraissent appartenir plutòt aux mers froides et orageuses qu'à celles des pays chauds, encore les espèces de ces dervières sont-elles bien plus petites, ce qui est le contraire de ce que l'on remarque ordinairement dans les autres mollusques.

G. CCCX. Bipapillaire, bipapillaria, Péron.

Anim. ovale, globuleux, terminé d'un côté par une sorte de pédoncule, et de l'autre par un renflement percé à l'extrémité de papilles coniques par deux orifices garnis chacun de trois tentacules roides, sétacés.

Ce genre très peu connu, et qui a été établi sur un mollusque observé à la Nouvelle-Hollande par Péron, u'est probablement qu'une Ascidie.

G. CCCXI. Fonie, fodia, Bosc.

Anim. ovale, mamelonné, partagé dans toute sa longueur par une cloison verticale qui contient l'estomac en deux tubes inégaux ouverts à chaque extrémité par un orifice, le supérieur un peu enfoncé et irrégulièrement denté; l'inférieur bordé d'un bourrelet circulaire formant ventouse, et servant à fixer l'animal.

Ce gen:c, qui n'est pas mieux connu que le précédent, a 
été établi par Bosc pour un mollusque des côtes de l'Amérique septentrionale; il se pourrait anssi que ce fût une Ascidie.

†† Ascidiens agrégés.

\section{G. CCCXII. Pyune, pyura.}

Animaux pyriformes, munis chacun de deux petits tubes courts, et contenus dans une loge particulière fermée par leur enveloppe extérieure, réunis au nombre de dix ou douze individus pour former une seule masse de forme variable, et sans ouvertures apparentes au-dehors.

C'est encore un genre qui demande de nouvelles obser vations.

\section{G. CCCXIII. Distome, distoma, Gaertner.}

Et Sigilline, Sav.

Anim. tuberculeux, mamelonnés ou coniques, à deux orifices chacun, rapprochés et garnis de six dents ou tentacules rayonnés, réunis en nombre plus ou moins grand.

Ce genre, composé de deux espèces seulement, l'une des mers de la Nouvelle-Hollande, et l'autre des côtes d'Angleterre, présente déjà le type de deux groupes.

$$
\text { I }{ }^{\mathrm{er}} \text { groupe. }
$$

Anim. dont la réunion forme un corps gélatineux, allongé, conique et subpédiculé. ( $\mathrm{G}$. Sigilline, Savigny.)

D. australis, etc.

$$
2^{\mathrm{e}} \text { groupe. }
$$

Anim. dont la réunion constitue des plaques ou des croûtes qui recouvrent les corps sous-marins.

D. variolatus, etc.

C'est à M. de Blainville que nous empruntons cette coupe et la caractéristique de quelques uns de ces genres. 
G. CCCXIV. Botryle, botryllus, Gaertner. Diazome, Polycline, Savigny; Polycycle, Botrylle, Lam.

Anim. ovales, plus ou moins aplatis, adhérens par leur face dorsale aux corps sous-marins, et par les côtés entre eux et en nombre plus ou moins grand, de manière à simuler un animal complexe, un peu variable; deux ouvertures bien évidentes aux deux extrémités du corps, sur chaque individu, l'une externe, pourvue de six papilles tentaculaires, l'autre interne subtubuleuse et plus petite.

Nous caractérisons ce genre, que nous n'avons point encore étudié, d'après M. de Blainville, et, comme lui, nous lui réunissons, sous forme de groupe, les genres Diazome, Polycline et Polycycle de MM. Savigny et de Lamarck, qui ne paraissent pas en différer essentiellement.

Voici la division de M. de Blainville :

$$
1^{\mathrm{e}} \text { groupe. }
$$

Animaux se groupant en cercles concentriques, de manière à constituer une masse orbiculaire, presque en forme de soucoupe. (Diazome.)

B. mediterraneus, etc.

$$
2^{\mathrm{e}} \text { groupe. }
$$

Animaux se disposant circulairement ou en rayonnant, souvent assez régulièrement autour d'un centre, de manière à former un ou plusieurs systèmes stelliformes enfoncés dans une masse gélatineuse horizontale.

Le corps comme divisé en trois loges. (Polycline.) B. violaceus, etc.

2) Le corps indivis, disposition en plusieurs cercles concentriques. (Polycycle.)

B. renierii, etc.

3) Le corps indivis; disposition rayonnée; huit tenta- 
cules, dont quatre plus petits à l'orifice externe. (Botrylle.)

B. stellatus, etc.

Les espèces de ce genre sont encore en petit nombre, et toutes des mers d'Europe; elles s'attachent sur différens corps, et particulièrement sur les plantes marines.

G. CCCXV. Syroïgue, synoicum, Phipps. Pulmonelle, Lam.; Euccelium, Didermum et Aplidium, Sav.

Anim. assez cylindriques, adhérens par la partie antérieure, et réunis entre eux par le côté de leur enveloppe extérieure, de manière à composer une seule masse variable dans sa forme; chaque individu ne présentant qu'une seule ouverture à l'extérieur, munie de six papilles tentaculaires, et conduisant dans la cavité intérieure, au fond de laquelle sont les deux véritables ouvertures pour la respiration et les déjections.

Nous ne connaissons pas encore assez ces animaux pour décider s'il convient d'en former plusieurs genres, comme l'a fait M. Savigny, ou si à l'exemple de M. de Blainville, il vaut mieux les réunir en un seul. Les bornes resserrées de ce travail ne nous permettant pas d'étendre beaucoup nos descriptions, nous nous contenterons de présenter les groupes établis par le dernier de ces sarans, et dans lesquels ils donnent suffisamment les caractères des différens genres dont il s'agit.

$$
\text { yer groupe. }
$$

Animaux réunis en une masse convexe, arrondie. ( $\mathrm{S}$. Alcyonaire; G. Pulmonelle, Lam.; Aplidium, Sav.) s. ficus, etc.

$$
2^{e} \text { groupe. }
$$

Animaux dont les corps horizontaux se réunissent en croûte mamelounée. (G. Fucoelium, Sav.)

S. subgelatinesum, etc. 
3 e groupe.

Auimaux dont les corps verticaux se réunissent aussi en croûte. (G. Didermum, Sav.)

S. fungosum, etc.

$$
4^{\mathrm{e}} \text { groupe. }
$$

Auimaux dont les corps fort longs, verticaux, se réunissent en espèce de cylindre, n'ayant qu'un seul orifice extérieur commun pour tous les individus.

$S$. turgens, etc.

Chacun de ces groupes ne contient encore qu'une espèce; c'est dans nos mers qu'elles ont toutes été observées.

$2^{\text {e FAMILLE. }}$

\section{LES SALPIENS, Blainv.}

Animal libre, errant, pélagien, en forme de tube plus ou moins cylindracé, ouvert aux deux bouts, ou a peu près; manteau épais, gélatineux, transparent, uni avec la tunique intérieure; les viscères formant un nucléus à l'intérieur, et près de l'une des extrémités; branchies en forme d'écharpe oblique et longitudinale.

Cette famille renferme des animaux uniquement Pélagiens, ne se fixant jamais aux corps, et flottant sans cesse à la surface de la mer où ils s'élèvent dans les temps calmes, et particulièrement sous les climats de la zone torride. Ils se meuvent par l'effet des contractions des bords de l'une des deux ouvertures, et, par ce moyen, acquièrent souvent assez de vitesse. Ce sont des animaux extrêmemeat mous, gélatineux et d'une transparence quelquefois égale au cristal le plus pur; plusieurs d'eutre eux se montrent réunis en société.

Les Salpiens présentent par leur singulière disposition la forme d'un tube, ouvert à peu près aux deux extrémités, et daus lequel l'eau circule librement pour baigner les branchies, porter la nourriture à l'animal et en même temps lui imprimer le mouvement. Quelques auteurs out 
différé d'opinion au sujet de ces deux ouvertures; les uns prétendent que celle qui est la plus éloiguée du nucléus, et par où s'introduit ordinairement l'eau, est la bouche de l'animal, tandis que d'autres y voient l'anus. M. de Blainville a observé, avec juste raison, que ni l'une ni l'autre n'est la bouche ou l'anus, car, comme l'a démontré $\mathrm{M}$. $\mathrm{Cu}$ vier dans un Mémoire anatomique sur les Biphores, la bouche et l'anus sont de très petites ouvertures, cachées dans le foud du vaste canal que forme l'enveloppe de l'animal; au surplus, on doit appeler antérieure, l'extrémité où se trouve le nucléus, et postérieure, celle qui lui est opposée. Nous avous remarqué que l'animal qui reçoit généralement l'eau par l'ouverture postérieure, peut aussi la recevoir par l'ouverture antérieure.

Nous admettons dans cette famille, comme daus la précédente, la division, proposée par M. de Blainville, des animaux simples et des animaux agrégés, parce que, quoiqu'il soit bien vrai que les Biphores se rémissent, soit dans une disposition linéaire, soit dans une disposition rayonnante, nous nous croyons assaré, après de nombreuses observations, que ce n'est que dans le jeune âge; mais il n'eu est pas de même du Pyrosome; les animaux qu'il comprend, sont probablement agrégés toute leur vie. Du reste, c'est sur des Hétérobranches que nous manquons le plus de renseignemens, et qu'il est peut-être le plus difficile de s'en procurer; ainsi donc tout notre trawail à leur sujet, qui n'est guère qu'une copie de cclui de M. de Blainville, n'est que provisoire.

I. Salpiens simples.

\section{G. CCCXVI. Brphore, salpa, Cuv.}

Anim. libre, très mou, transparent, allongé, plus ou moins cylindrique ou en forme de prisme, souvent muni en avant, et rarement en arrière, d'appendices tentaculiformes allongés et coniques; manteau ample, gélatineux, musculaire enveloppant, muni quelquefois de tubercules; deux ouvertures terminales ou subterminales, l'une plus grande que l'autre, transverse, souvent munie d'une sorte de 
lèvre mobile; viscères formant un nucléus presque toujours coloré à la partie antérieure et intérieure; branchies en forme d'écharpe finement striée en travers, se portant obliquement du nucléus à la partie postérieure; bouche au côté inférieur du nucléus; anus plus en arrière, toujours clans l'intérieur du manteau.

Chaque espèce présentant un mode de réunion particulier pour un plus ou moins grand nombre d'individus pendant le jeune âge.

Ce genre renferme un très grand nombre d'espèces qui s'augmente tous les jours par les décourertes des voyageurs; mais il en est plusieurs qui sont très incomplétement connues, et d'autres qui sont certainement des doubles emplois, la même espèce ayant souvent été observée à des âges différens. Nous ne faisons point connaître ici la manière dont nous distribuons les espéces de ce beau genre pour lequel nous avons réuni un grand nombre de dessins, ve voulant le faire que lorsque nous aurons terminé un travail spécial que nous poursuivons depuis quelque temps, et pour lequel il nous manque encore quelques vérifications de détail sur les animaux vivans.

Les Biphores sont phosphoriques; ils habitent les climats chauds et les climats tempérés, mais particulièrement les premiers. On les aperçoit toujours près de la surface de la mer dans les temps calmes, et, comme l'ont très bien observé plusieurs naturalistes, quelques espèces affectent une manière particulière de s'agglomérer; mais ce n'est que pendant leur jeunesse, car plus tard on les retrouve isolés. Les uns se réunissent au nombre de cinq à seize pour former une sorte de rosace; d'autres se réunissant par toute leur longueur, forment ainsi des rubans quelquefois très allongés, et dans lesquels chaque individu présente une direction transverse. Il en est encore qui forment aussi deux rangées accolćes par le dos, de manière que chaque individu d'une rangée correspond entre deux individus de l'autre; enfin il en est qui forment des chainons dans lesquels chaque individu est étendu dans le sens de la longueur et attaché à celui qui le précède et à celui qui le suit par un côté différent et une partie de sa longueur; ces animaux varient 
ainsi leur manière de s'agréger, mais toujours de façon que les deux ouvertures soient libres. Un fait sur lequel il est, sans doute, important d'insister pour prévenir les voyageurs contre des erreurs qu'il serait très facile de faire, c'est que les individus jeunes diffèrent beaucoup quelquefois de ceux qui sont adultes, et nous en connaissons une espèce qui, lorsque nous l'avons observée agrégée, ne présentait que de faibles indices des appendices tentaculaires, tandis que, libre, elle montrait ces organes très allongés.

Cette singulière agrégation des Biphores mérite certainement de fixer d'une manière particulière l'attention des maîtres de la science; elle rappelle celle de ces zoophytes qui habitent avec les Biphores au milieu de l'Océan, qui naissent et vivent quelque temps ainsi réunis en groupes déterminés pour chaque genre, mais qui se détachent et deviennent indépendans sitôt qu'ils ont atteint toute leur croissance. Ce rapprochement doit inviter les naturalistes qui rencontrent ces derniers animaux à en observer l'organisation avec une scrupuleuse attention, car nous ne serions pas étonné que quelques uns d'cux dussent s'élever un peu plus haut dans l'échelle des êtres.

\section{G. CCCXVII. Trmontenne, timoriensis, Quoy et Gaim.}

Anim. libre, allongé, gélatineux, cylindrique antérieurement, triangulaire et pointu postérieurement; muni d'un appendice labial et d'un tube digestif à deux ouvertures; point de nageoires ni de branchies; peut-être des yeux?

Ce genre, très ircomplétement connu, pent fort bien appartenir à la famille des Salpiens. Cependant il rappelle singulièrement les Firoles de l'ordre des Nucléobranches. On serait tenté de le prendre pour un de ces animaux mutilés, comme il arrive si souvent d'en rencontrer; nous le conserverous cependant jusqu'à nouvel ordre; car les savans voyageurs à qui nous le devons nous rapporteront peut-être de lcur seeond voyage de nouveaux renseignemens à son sujet. 
La T. triangularis est de Timor, et c'est la seule espèce counue.

G. CCCXVIII. Monophore, monophorus, Quoy et Gaim.

Anim. Corps libre, gélatineux, transparent, allongé, pyramidal, un peu aplati; arrondi à une de ses extrémités, pointu à l'autre; ayant un tube digestif, à une seule ouverture munie de deux lèvres ou valvules; des yeux?

Telle est la caractéristique de ce genre établi par MM. Quoy et Gaimard pour un mollusque qu'ils ont rencontré dans la mer des Iudes. Ce n'est qu'arec doute que nous le rapportons à cette famille; cependant l'embarras où nous sommes pour le placer convenablement, nous a fait prendre ce parti qui a déjà été inđiqué par M. de Blainville, surtout en considérant certains caractères d'ensemble, et en supposant que la seconde ouverture aura échappé à l'investigation des deux observations. Peut-être ce mollusque devra-t-il être rapproché des Ptérosomes de M. Lesson.

G. CCCXIX. Phyluró, phylliroe, Péron et Lesu.

Anim. pélagien, très comprimé latéralement; terminé en arrière par une sorte de nageoire verticale; tête assez distincte et pourvue d'une paire d'appendices tentaculiformes, servant peut-être à la natation; une ouverture subterminale, en fer à cheval, et située à l'extrémité d'une trompe courte et rétractile; anus au côté droit antérieur du corps; orifice des organes de la génération uniques, du même côté et en avant de l'anus; branchies?

Le geure Phylliroé a été découvert et établi par Péron et Lesueur sur un individu dont ils n'ont pas eu une connaissance suffisante; depuis lors il n'a été retrouvé, à ce que nous sachions, que par M. de Lapilaye qui en a fait lithographicr une figure assez bonne, mais encore incom- 
plète, puisque les branchies n'y sont point indiquées. Ce que nous savons de plus important sur ce mollusque est donc dû à M. de Blainville qui a pu, jusqu'à un certain point, l'étudier, puisqu'il en possède un dans sa collection. On ne connaît encore que le $P$. bucephalum; cependant il parait que MM. Quoy et Gaimard viennent d'en découvrir une seconde espèce.

L'incertitude qui règne encore sur ce mollusque, nous engage, ainsi que la considération de quelques uns de ses caractères, à le placer provisoirement dans les Salpiens. Peut-être devra-t-il, quand il sera mieux connu, être reporté plus haut; mais certainement ce ne sera ni arec les Ptéropodes, ni avec les Nucléobranches.

†† Salpiens agrégés.

G. CCCXX. Руколоме в prosoma, Péron.

Animaux allongés, fusiformes, terminés en pointe d'un côté, et obtus de l'autre, munis de deux ouvertures, l'une externe non terminale, l'autre interne et terminale, réunis entre eux vers leur base par le moyen de leur enveloppe ex térieure, de manière à composer des anneaux assez nombreux et réguliers qui concourent à former un long cylindre, libre, hérissé à l'extérieur, creux et mamelonné à l'intérieur, ouvert seulement à une extrémité.

Ce genre reuferme trois espèces de la Méditerranée et des mers chaudes, mais que l'on ne rencontre qu'à une assez grande distance des rivages. Ils sont phosphoriques, et répandent la auit, pendant les temps calmes, une très vive lumière. 


\section{CINQUIĖME CLASSE.}

\section{CIRRIPÈDES.}

Cirrhopodes, Cuv., Fér.; Cirrhipèdes, Lam.; Nématopodes, Blainv.; Cirripèdes, Lat.

Anim. plus ou moins conique, quelquefois un peu comprimé, enveloppé d'un manteau en forme de sac ouvert seulement en arrière, renflé à la partie antérieure qui, d'après la position naturelle du mollusque, est l'inférieure; terminé supérieurement par un certain nombre de paires de cirrhes longs, cornés, articulés, ciliés, et se courbant au sommet; la tête non distincte, sans yeux ni tentacules; la bouche munie de mâchoires latérales, cornées, dentées et articulées; des branchies paires situées de chaque côté, à la base des premiers cirrhes; orifice de la génération à l'extrémité d'un tube charnu s'élevant du milieu des derniers cirrhes; anus médian à la base de ce tube.

Coq. assez variable, mais toujours composée de plusieurs valves soudées ou non soudées ensemble; dans un seul cas point de coquille, mais alors une enveloppe molle qui en tient lieu; adhérente immédiatement, ou au moyen d'un pédicule charnu qui provient du manteau.

Marins.

Cette classe, peu nombreuse en genres, renferme des animaux de toutes les mers. 
$1^{\text {re FAMIILE. }}$

LES LÉPADIENS, Blainr.

Cirripèdes pédonculés, Lam.; Anatifes, Fér.; Gymnodermes et Ostracodermes, Lat.

Anim. ovale, comprimé, suspendu dans son enveloppe testacée, à laquelle il adhère par un muscle transverse situé près de l'ouverture; manteau ouvert seulement en arrière, et inférieurement, se liant au bord de la coquille, et se prolongeant à l'autre côté en un pédicule contractile et charnu, adhérent par son extrémité aux corps sous-marins; branchies en forme de pyramides plus ou moins nombreuses, adhérentes en dehors de la base des cirrhes.

Coq. presque toujours composée de cinq valves principales, rarement sans aucun vestige de valves, mais alors celles-ci étant remplacées par une enveloppe subcartilagineuse et épaisse; valves principales triangulaires, minces, se touchant ou s'imbriquant par les bords, quelquefois rudimentaires, et alors très écartées; disposées de la manière suivante, deux latérales supérieures assez grandes, et recevant le muscle transverse de l'animal, deux latérales supérieures plus petites, et une médiane servant à les lier ensemble; souvent un nombre plus ou moins grand de pièces accessoires fixées à la base.

Cette famille comprend des genres fort remarquables, non seulement par l'organisation des animaux, mais encore par celle des coquilles et de leur pédoncule. Les Lépadiens se fixent aux corps sous-marins par le moyen de ce pédoncule, et forment ainsi des groupes très nombreux, s'élevant ou descendant selon la position de ces corns, et divergens dans différentes directions. Ils habitent particulièrement sur les rivages parmi les rochers; mais on en rencontre aussi en pleine mer fixés aux corps flottans, surtout aux fucus et 
orrans ariec eux à la surface des eaux; il y en a mếme nne espèce, le Cineras parasita de M. Lesson, dont nous faisons le genre Alèpe, qui n'a encore été rencontrée que sous l'ombrelle d'une espèce de Méduse; il en est aussi que nous arons trouvés sur la coquille des Janthines. Ces mollusques sont répandus dans toutes les mers, et il n'est pas extraordinaire de rencontrer la même espèce dans des lieux fort éloignés, car les navires en ont presque toujours leur carène plus ou moins chargée, et par conséquent doivent les dépayser souvent. Les noirs de l'île de Gorée mangent une grosse espèce d'Anatife qui est, en effet, assez délicate.

On ne connaît point encore de coquilles fossiles appartenant à cette famille.

\section{G. CCCXXI. Alèpe, alepas, Rang. Triton, Lin.}

Anim. ovale, comprimé, fabiforme, arrondi près dư pédicule; celui-ci médiocrement allongé; cirrhes un peu courts, se recourbant à peine à leur sommet, et composés d'environ dix à douze articles hispides à leur base.

Coq. remplacée par une enveloppe d'une seule pièce épaisse, subgélatineuse et un peu diaphane, sans autre ouverture que celle qui sert au passage des cirrhes, se continuant avec le pédicule, et ne présentant aucune trace de pièces testacées.

Linné avait connu, sans doute, ce mollusque puisqu'il avait établi Je genre Triton pour un Anatife sans coquille; mais, n'ayant plus été retrouvé, on abandonna ce genre, et son nom fut donné à des mollusques qui font partie de la classe des Gastéropodes. Les voyages de MM. Lesson et Garnot, Quoy et Graimard, viennent de faire connaître les Tritons de Linné, et déjà ils se trouvent publiés par ces estimables naturalistes dans deux genres différens et sous deux dénominations spécifiques différentes. M. Lesson en fait son Cineras parasita, et MM. Quoy et Gaimard, l'Anatifa univalvis. Nous pensons qu'il diffère plus des Cinéras, que les Cinéras ne diffèrent des véritables Anatifes, et que, par conséquent, c'était avec raison que Linné en avait 
fait un genre distinct; nous proposons donc le genre Alèpe pour ce singulier mollusque, et nous conserverons à l'espèce, qui lui sert de type, Ie nom spécifique d' $A$. parasita, celui d'univalvis devenant un caractère générique.

Ce riollusque a été trouvé sur l'ombrelle d'une Méduse; et il n'y a pas de doute qu'il ne soit tout-à-fait Pélagien. Cette enveloppe presque gélatineuse et sa transparence en sont la preuve.

Ce genre et le suivant lient parfaitement la dernière famille des Acéphales avec la première des Cirripèdes.

\section{G. CCCXXII. Gymnolèpe, gymnolepas, Leach.} Otion et Cinéras, Leach.; Aurifère, Blainv.

Anim. assez peu comprimé, avec les cirrhes plus recourbés à leur extrémité; manteau presque complétement nu, épais et subcartilagineux; pédicule long et épais.

Coq. rudimentaire, composée de petites valves très écartées.

Les Gymnolèpes paraissent habiter Ies mers du Sénégal et, dit-on aussi, celles du Nord. Nous ne les avons jamais rencontrées que sous la carène des bâtimens, et nous pensons qu'elles ne doivent point se fixer sur les rochers, comme font généralement les Lépadiens. Ce genre en comprend deux de M. Leach, que nous distinguerons comme groupes.

$$
\text { I }{ }^{\text {er }} \text { groupe. }
$$

Deux tubes aurifurmes au summet; deux petites valves distinctes. (Otion, Leach; Aurifère, Blainv.)

G. cuvierii, etc.

\section{$2^{\mathrm{e}}$ groupe.}

Point de tubes auriformes; la forme plus angulease; cinq petites valves distinctes. (Cinéras, Leach.)

G. cranchii, ete. 
G. CCCXXIII. Anatife, anatifa, Lam. Pentalasmis, Leach.; Pentalèpe, Blainv.

Anim. assez comprimé, enveloppé d'un manteau très mince ; pédicule souvent très allongé ; cirrbes contournés au sommet.

Coq. subtriangulaire, anguleuse, formée de cinq valves très distinctes, enveloppant complétement l'animal.

Les Anatifes sont très répandus, mais particulièrement sur les côtes d'Afrique; il en est aussi qui n'habitent que les hautes mers. Ils se groupent par bouquets quelquefois très grands, et se suspendent souvent ainsi à la carène des bâtimens. Nous en avons plusieurs espèces sur nos côtes.

\section{G. CCCXXIV. Pollicip̀̀De, pollicipes, Lam. Pentalèpe, Blainv.}

Anim. semblable à celui des Anatifes, mais ayant généralement le pédicule plus court.

Coq. subtriangulaire, composée, outre les cinq valves principales, d'un nombre plus ou moins grand de. petites pièces testacées fixées à leur base.

Ce genre, fort voisin, sans doute, des Anatifes, s'en distingue très bien par la présence d'un grand nonibre de petites pièces qui accompagnent les cinq valves ordinaires. On en connaît seulement quelques espèces.

G. CCCXXV. Polylèpe, polylepas, Blainv. Scalpellum, Leach.

Anim. semblable à celui des Anatifes, ayant le pédicule plus ou moins allongé et squameux.

Coq. formée de treize valves recouvrant complétement l'animal; six valves principales, une dorsale, une ventrale et deux paires de latérales.

Ce genre, que nous ne connaissons pas, renferme trois ou quatre espèces d'après $M$. de Blainville. 


\section{G. CCCXXVI. LiтholÈpe, litholepas, Blainv. Lithotria, Sow.}

Anim. comprimé.

Coq. irrégulièrement subpyramidale, comprimée, portée à l'extrémité d'un pédicule tubuleux, tendineux, ayant à sa base un appendice testacé ressemblant à une patelle renversée, formée de huit valves contiguës, inégales; six latérales, dont les inférieures très petites; une dorsale, grande, ligulée, et une ventrale également très petite.

Ce genre, d'abord établi sous un nom par Sowerby, et ensuite adopté sous un autre par M. de Blainville, n'est, selon ce dernier savaut, qu'un véritable Anatife qui se serait fixé sur une valve de Vénérupe dans le fond d'une de ces cavités que creusent ordinairement ces coquilles. Il n'est encore question que d'une seule espèce.

\section{$2^{\text {e FAMILLE. }}$}

\section{LES BALANIDES, Blainv.}

Cirripèdes sessiles, Lam.; Balanes, Fér.; Quadrifores et Bifores, Lat.

Anim. conique, quelquefois très déprimé, et quelquefois aussi presque cylindrique, du reste semblable à celui de la famille précédente, mais sans pédicule, et ayant les branchies en forme de deux ailes frangées attachées à la face interne du manteau. Coq. épaisse, solide, de forme assez variahle, mais cependant toujours conique ou subcylindrique, adhérente par sa base aux surfaces, ou pénétrante; composée d'un cône formé d'une ou de plusieurs pièces réunies latéralement, ouvert à sa base, ou fermé par une pièce membraneuse ou calcaire, servant à l'adhérence; toujours ouverte à son sommet, mais munie dans cette partie d'un opercule pyramidal à deux ou quatre valves.

\section{Marins.}


Cette famille, fort intéressante par la singulière conformation des coquilles qu'elle comprend, est encore incomplétement connue, à cause de la difficulté qu'il y a à se procurer à la fois toutes les pièces d'un même individu. On y a créé bien plus de genres que nous en présentons, et cependant nous sommes très porté à croire qu'il sera encore nécessaire d'en réunir quelques uns; nous avons adopté des divisions qui faciliteront la détermination des genres; cependant il se pourrait qu'on dût supprimer celles qui ont pour base le nombre des pièces de l'opercule, parce qu'il est probable que ce nombre est toujours de quatre, mais. que quelquefois les deux pièces d'un même côté sont comme soudées, et paraissent alors n'en faire qu'une.

Le cône des Balanides est remarquable par les cavités tubuleuses dont il est percé dans la longueur, et qui s'ouvrent à la base où elles sont très sensibles; ce caractère est unique dans les mollusques, et contribue à distinguer cette famille. M. de Férussac a fait, dans le Dictionnaire classique d'Histoire naturelle, ưn article extrêmement intéressant sur la singulière organisation de ces coquilles, et qui a beaucoup éclairé la science à leur sujet.

† Cône univalve.

\section{G. CCCXXVII. Pyrgome, pyrgoma, Sow. Et Boscie, Fér.}

Anim. inconnu.

Coq. épaisse, en cône plus ou moins élevé ou surbaissé ; indivise, rayonnée du sommet à la base; support calcaire en forme de calice ou de tube, fermé, pénétrant dans les madrépores; ouverture petite; opercule formé de deux pièces longues et allongées.

Ce genre, encore peu connu, renferme plusieurs cspèces; nous en avons examiné une, et il nous a semblé que l'opercule était quadrivalve; si cette observation se confirmait, il ne faudrait former qu'un seule groupe dans cette section. M. de Férussac, qui a établi le genre Boscie, pense luimếme qu'il se rapporte à celui-ci.

†† Cône à quatre valves.

a. Opercule bivalve. 
G. CCCXXVIII. VRRRe, verruca, Schum. Ochthosie, Ranzani.

Anim. inconnu.

Coq. subconique, verruqueuse, à quatre valves inégales, une dorsale (la plus petite), une ventrale (la plus grande), et deux latérales semblables; une lame interne quadripartite, dont trois portions divisent la cavité en trois loges; support membraneux; ouverture trigone, oblongue; opercule pyramidal, articulé, bivalve.

Adoptant les idées de M. de Blainville au sujet des Ochthosies, nous pensons que les cônes de ces coquilles sont munis de quatre valves et non de trois. On ne connait qu'une s’uule espèce, la $V$. stroemii.

b. Opercule quadrivalve.

G. CCCXXIX. Creusie, creusia, Leach.

Anim. subglobuleux, ayant trois ou quatre paires de bras tentaculiformes; bouche sans saillie. Coq. patelliforme, assez mince, à quatre valves, ayant un support calcaire, conique, très profond, et pénétrant dans les madrépores; ouverture petite, ovale; opercule assez grand, subpyramidal et quadrivalve.

Ce genre, peu riche encore en espèces, ne se rencontre que dans les Polypiers où il pénètre souvent à d'assez grandes profondeurs. Nous en possédons de fossiles qui ont été trouvés par M. Charles Des Moulins dans ceux des environs de Bordeaux. Les espèces vivantes sont des pays chauds.

G. CCCXXX. Conne, conia, Leach. Et Azemus, Ranz.

Anim. conique, du reste comme dans la caractéristique de la famille.

Coq. patelliforme, à quatre valves plus ou moins distinctes, presque égales, et ordinairement striées 
de la base au sommet; support plat, fort mince ou membraneux; opercule pyramidal, quadrivalve, articulé.

Ce genre renferme cinq à six espèces.

†† Cône à six valres.

a. Opercule composé de pièces rudimentaires non articulées.

\section{G. CCCXXXI. Tubicinelle, tubicinella; Lam.}

Anim. subcylindrique, assez allongé, du reste comme dans la caractéristique de la famille.

Coq. peu épaisse, subcylindrique, tubuleuse, élevée, à six valves quadrangulaires; entourée de bourrelets annuliformes, tronquée aux deux extrémités; deux ouvertures rondes, égales, l'inférieure fermée par une membrane, la supérieure munie d'un opercule à quatre pièces obtuses, jointes à ses bords par une membrane assez considérable.

On ne connaît qu'une seule espèce de Tubicinelle, la $T$. balcenarum, qui pénètre dans la peau des baleines, et la remplit quelquefois d'une manière fort extraordinaire. Ce genre est certainement peu distinct des Coronules.

G. CCCXXXII. Coronule, coronula, Lam. Et Chélonobie, Leach.; Cétopire, Ranz.; Diademe, Schum.

Anim. assez déprimé, peu élevé, du reste comme dans la caractéristique de la famille.

Coq. généralement peu élevée, et de forme assez variable, à six valves très régulièrement disposées; aucune trace de support; opercule non articulé, formé de deux paires de petites valves plates, minces, jointes à l'ouverture du tube par une membrane considérable.

Le genre Coronule renferme quelques espèces bien dis-tinctes les unes des autres, et dont quelques auteurs ont cru deroir faire des genres à part; mais qui ne sauraient tout au plus constituer que des groupes, encore convien- 
draituil pour les établir d'avoir sous les yeux un plus grand nombre d'espèces que celui qu'on connaît. Les mollusques des Coronules vivent comme ceux des 'Tubicinelles sur les animaux marins dans la peau desquels ils s'introduisent; il y en a aussi qui se fixent sur les pierres et les grosses coquilles.

b. Opercule quadrivalve, articulé.

\section{G. CCCXXXIII. Chтuamale, chthamalus, Ranz.}

Anim. comme dans la caractéristique de la famille. Coq. très déprimée, à six valves très épaisses à leur base, et formant des rayons très réguliers; lame interne courte; support membraneux; ouverture tétragone à cotés presque égaux; opercule faiblement pyrainidal, quadrivalve, lié au bord de l'ouverture par une membrane.

Ce genre est très peu connu; il appartient à la Méditerranée.

\section{G. CCGXXXIV. Balane, balanus, Brug.}

Anim. conique, du reste comme dans la caractéristique de la famille.

Coq. conique, souvent infléchie, plus ou moins élevée, formée de six valves toujours très distinctes, une ventrale, une dorsale, et deux paires de latérales; un support calcaire, plat, assez épais, ou point de support; opercule en forme de pyramide un peu oblique, articulé et composé de quatre pièces triangulaires, dont deux, les plus petites, présentent un cuilleron droit et aplati.

Les Balanes s'attachent à la surface des corpls sous-marins, mais ne pénètrent jamais dans ces corps comme quelques uns des genres précédens. Le nombre des espèces est assez considérable, car il en existe dans toutes les mers. On les trouve toujours réunies par groupes considérables, tapissant les rochers, et se pressant les unes contre les autres, de manière à irrégulariser leur forme. On peut établir deux coupes principales d'après la considération du support. 
1 er groupe.

Support nul ou membraneux.

B. spinosus, etc.

$2^{\mathrm{e}}$ groupe.

Support considérable et calcaire.

$B$. gigas, etc.

Nous en possédons plusieurs espèces sur nos cótes; il y en a aussi un bon nombre à l'état fossile. Lorsque les $\mathrm{Ba}$ lanes sont très jeunes, leur coquille ne consiste presque que dans l'opercule.

\section{G. CCCXXXV. Acaste, acasta, Leach.}

Anim. comme dans la caractéristique de la famille. Coq. conique, composée de six valves assez distinctes;

support calcaire, également conique et renversé, pénétrant dans les éponges; opercule quadrivalve.

Certainement ce genre est bien peu distinct des Balanes; aussi nous ne l'aurions pas conservé, s'il n'affectait pas cette habitude de se loger dans les éponges, ce qui n'arrive jamais aux Balanes, qui se fixent toujours à la surface des corps. On en connaît plusieurs espèces qui sont des pays chauds.

†††† Cône à huit valves.

G. CCCXXXYI. Ocromìne, octomeris, Sow.

Anim. inconnu.

Coq. subconique, à huit valves inégales; support?

opercule à quatre pièces, deux de chaque côté.

M. Sowerby a établi ce genre pour une Balanide du cap de Bonne-Espérance, l'O angubra. 


\section{GENRES NON GLASSÉS.}

Plusieurs genres décrits dans ces derniers temps, et que nous n'avons point eu occasion d'observer par nous-même, nous laissent dans une grande incertitude sur la place qu'ils doivent occuper dans la classification que nous avons suivie, parce que leurs caractères n'ont généralement pas été présentés avec assez de précision ou de détails. Nous les signalerons en attendant de plus amples renseignemens à leur sujet, et afin de fixer sur eux l'attention des naturalistes.

G. Pyrgo, pyrgo, Def.

Anim. inconnu.

Coq. presque microscopique, sphéroïdale, régulière, formée de deux pièces ou valves presque séparables, égales, se joignant dans toute leur circonférence, si ce n'est en avant, où est une petite ouverture étroite, transversale.

M. Defrance, qui est l'auteur de ce genre, le range parmi les Sphérulacés, tandis que M. de Blainville le met immédiatement après les Cymbulies.

G. Denmatobranche, dermatobranchus, Van-Hasselt.

Anim. déprimé, semi-circulaire, pourvu en dessous d'un pied assez large, fort distinct, recouvert en dessus d'un manteau élargi, arrondi en avant, rétréci en arrière, hérissé de stries ou de pustules allongées, branchiales? une paire de tentacules courts, rapprochés, contractiles, situés entre la tête et le manteau; yeux nuls? trois ouvertures au côté droit du corps, l'antérieure près la tête, pour l'appareil générateur, la seconde pour l'anus, et la troisième pour l'organe urinaire.

M. Van-Hasselt a établi ce genre pour des mollusques 
qu'il range dans les Nudibranches, et M. de Blainville croit qu'il convient de les mettre à côté des Scyllées.

\section{G. Truodine, tylodina, Raf.}

Anim. gastéropode, à petite coquille dorsale, extérieure, membraneuse, ovale, patelliforme, sans spire, à sommet calleux; quatre tentacules, dont les deux postérieurs éloignés des antérieurs, et plus grands qu'eux; branchie dorsale sous la coquille, à droite; anus à la droite du cou.

M. de Blainville place ce genre à côté des Siphonaires et des Ombrelles.

\section{G. Atras, atlas, Lesu.}

Anim. partagé en deux parties réunies par une sorte de pédoncule, à peu près comme dans le Gastéroptère; la postérieure ovalaire, l'antérieure dilatée circulairement, et ciliée sur ses bords, mais pourvue d'un très petit pied distinct en dessous, et d'une paire de très petits tentacules auriformes en dessus; I'anus au milieu du côté droit de la masse postérieure; les organes de la respiration inconnus, ainsi que la ierminaison de ceux de la génération.

M. Lesueur pense que les cils qui bordent la partie antérieure sont les organes de la respiration, et M. de Blainville croit, au contraire, que les branchies doivent être situées au côté droit, près de l'anus. Ce naturaliste place le genre Atlas à côté des Gastéroptères dans les Acères.

\section{G. Pleurocìre, pleurocerus, Raf.}

Et Oxitrème, Raf.

Anim. ayant une tête proboscidiforme; deux tentacules latéraux, subulés, aigus, portant les yeux à leur base externe.

Coq. ovale ou pyramidale; ouverture oblongue, 
ayant la lèvre extérieure mince, l'interne appliquée contre la columelle; celle-ci lisse, torse et sans ombilic.

Operc. corné ou membraneux.

M. de Blainville place ce genre à côté des Hélicines, et le divise en deux groupes, dont l'un comprend l'Oxitrème de M. Rafinesque, établi pour une coquille qui se termine à l'extrémité antérieure de son ouverture par une longue pointe aiguë. M. de Blainville pense aussi que le genre Pleurocère pourrait bien n'être que la Paludine coupée de M. Say.

\section{G. Oxınó́, oxinoe, Raf.}

Anim. gastéropode à grande coquille dorsale, antérieure, bulliforme, à spire simple; pied étroit; branchies marginales, striées transversalement; manteau élargi en deux ailes latérales; deux tentacules non rétractiles.

M. Rafinesque prétend que ce genre ne diffère des Sigarets qu'en ce que la coquille est extérieure, tandis que celle de ces mollusques est intérieure, et $M$. de Blainville ajoute, avec raison, que si les branchies sont placées comme il le dit, la différence est encore plus grande.

Nota. M. de Férussac vient de nous montrer aujourd'hui même des mollusques qui lui ont été envoyés de la Méditerranée, et dans lesquels nous ne balançons pas à reconnaître avec lui le genre Actéon d'Ocken, ou Elysie de Risso. Il nous a fait remarquer que les branchies tapissent le dos et la surface supérieure des lobes, sous la forme de réseau vasculaire; ainsi ce genre viendrait se ranger dans notre famille des Placobranches, qui ne renferme encore qu'un seul genre. 


\section{EXPLICATION DES PLANCHES.}

\section{Ire CLASSE. CÉPHALOPODES.}

Peanche I.

Cryptodibranches. - Fig. 1. Argonaute; $b, b$, bras; $c$, bouche; $d$, yeux; $e$, bras élargis par des membranes véliformes; $g$, tube conduisant l'eau dans la cavité branchiale; $h$, coquille enveloppante. Fig 2. Onychoteuthe; $a$, nageoires; $b$, bras; $c$, bouche; $d$, yeux; $e$, bras pédonculés; $m$, manteau. Fig. 3. Coquille interne de ce dernier.

Siphonifères. - Fig. 4. Spirule; $b$, bras; $c$, bouche; $d$, yeux; $e$, bras pédonculés; $m$, manteau; $h$, coquille. Fig. 5. Coquille interne de cette dernière; $l$, dernière loge; $n$, siphon; $p$, indication des différentes cloisons. Foraminifìres. - Fig. 6. Anomaline; $k$, ouverture de la coquille.

\section{C CLASSE. PTÉROPODES.}

\section{Planche II.}

Fig. r. Cymbulie; $a$, nageoires; $b$, lobe intermédiaire; $i$, viscères vus à travers la coquille; $h$, coquille. Fig. 2. Hyale; $a$, nageoires; $b$, lobe intermédiaire; $c$, bouche; $e$, expansions latérales du manteau; $i$, viscères vus à travers la coquille; $h$, coquille. Fig. 3. Créséis; $a$, nageoires; $b$, lobe intermédiaire; $c$, bouche; $i$, viscères vus à travers la coquille; $h$, coquille. Fig. 4 . Cuviérie; $a$, nageoires; $b$, lobe intermédiaire; $c$, bouche; $e$, branchies; $i$, viscères vus à travers la coquille; $f$, ovaires; $g$, cœur; $h$, coquille; $d$, cavité postérieure de la coquille. Fig. 5. Clio; $a$, nageoires; $b$, expansions ventrales correspondant au lobe intermédiaire dans les Ptéropodes testacés; $c$, bouche; $i$, viscères vus à travers le manteau; $k$, tentacules; $l$, tête. 
PI.T.

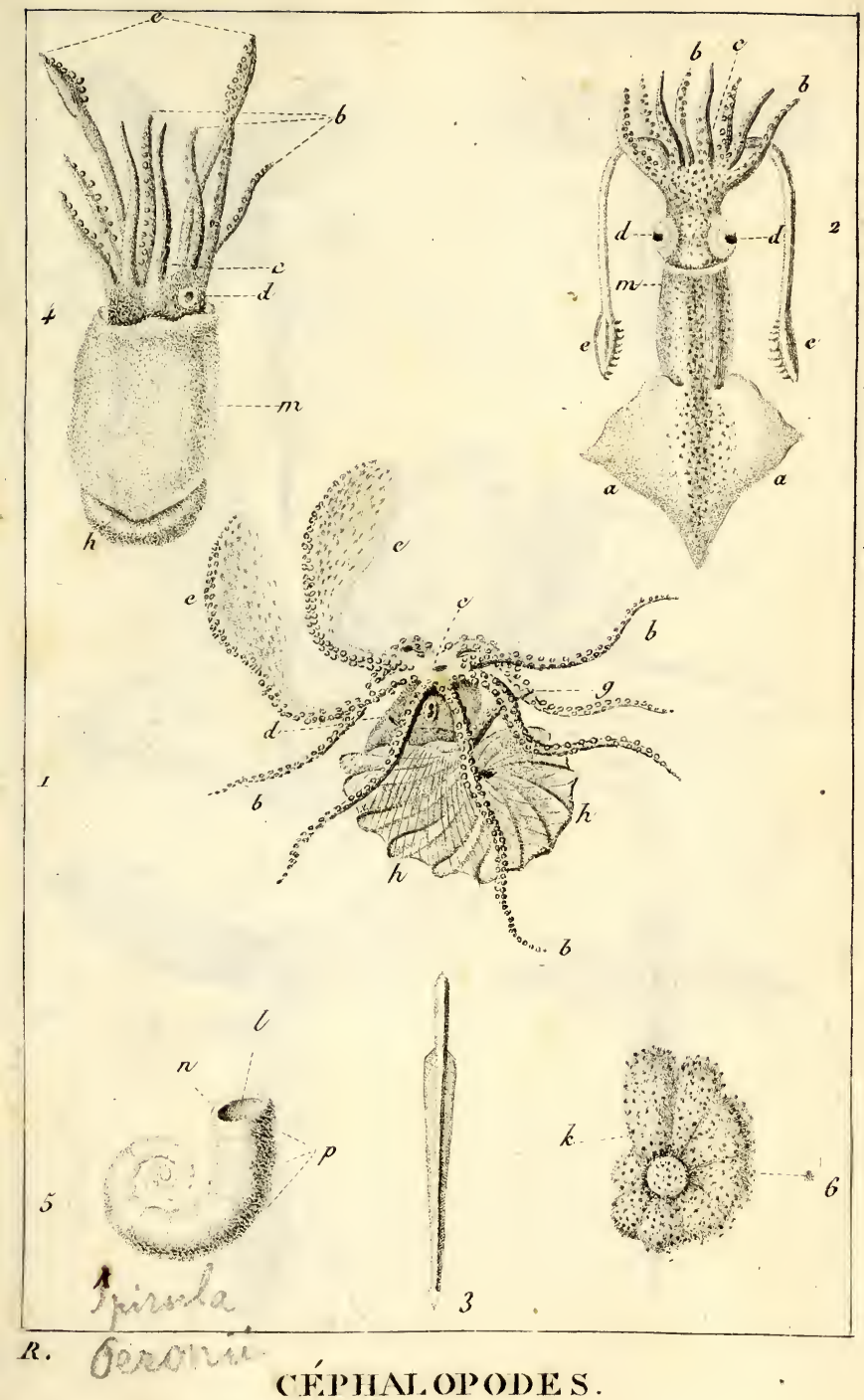



PI.II.

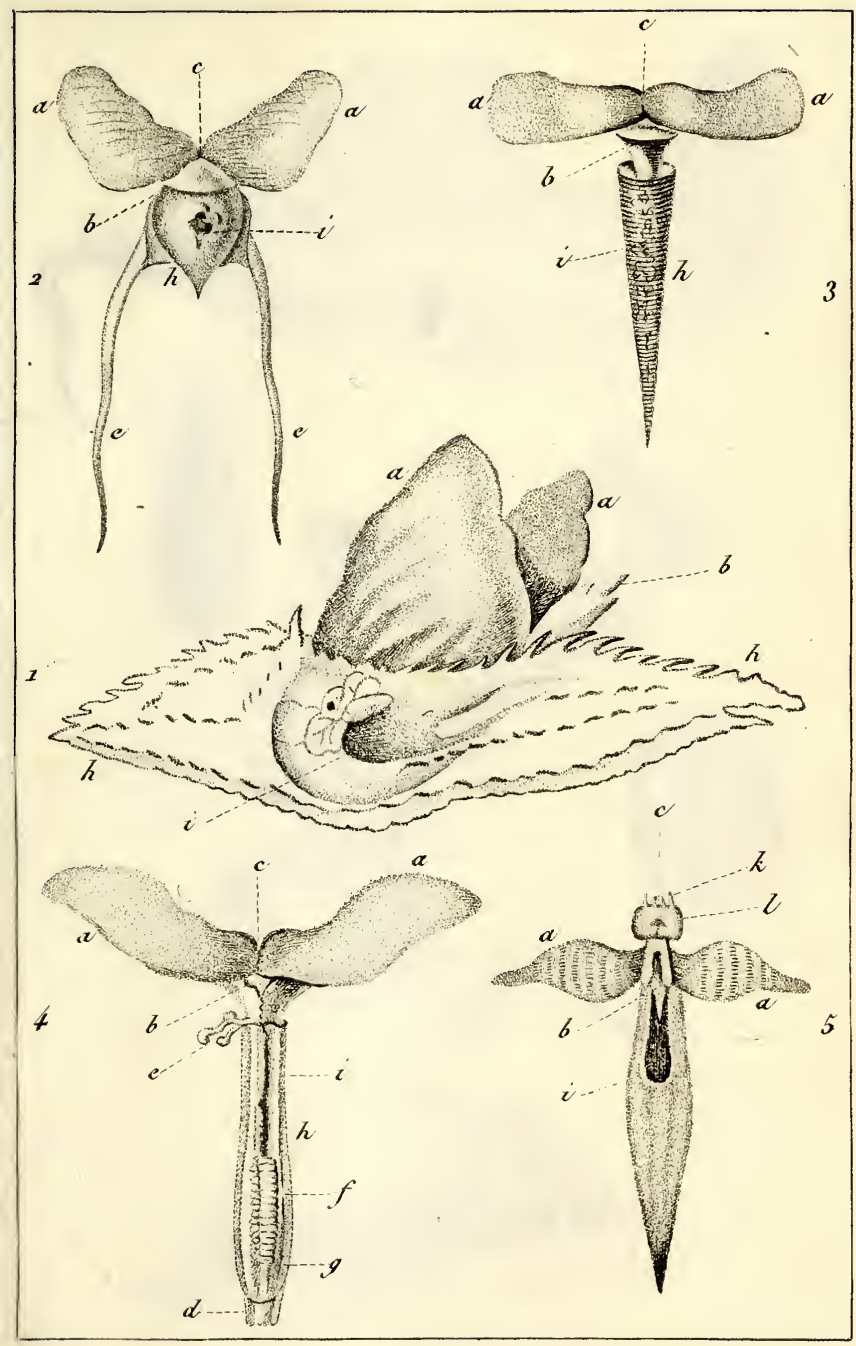

$R$.

JTEROPODES. 


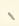




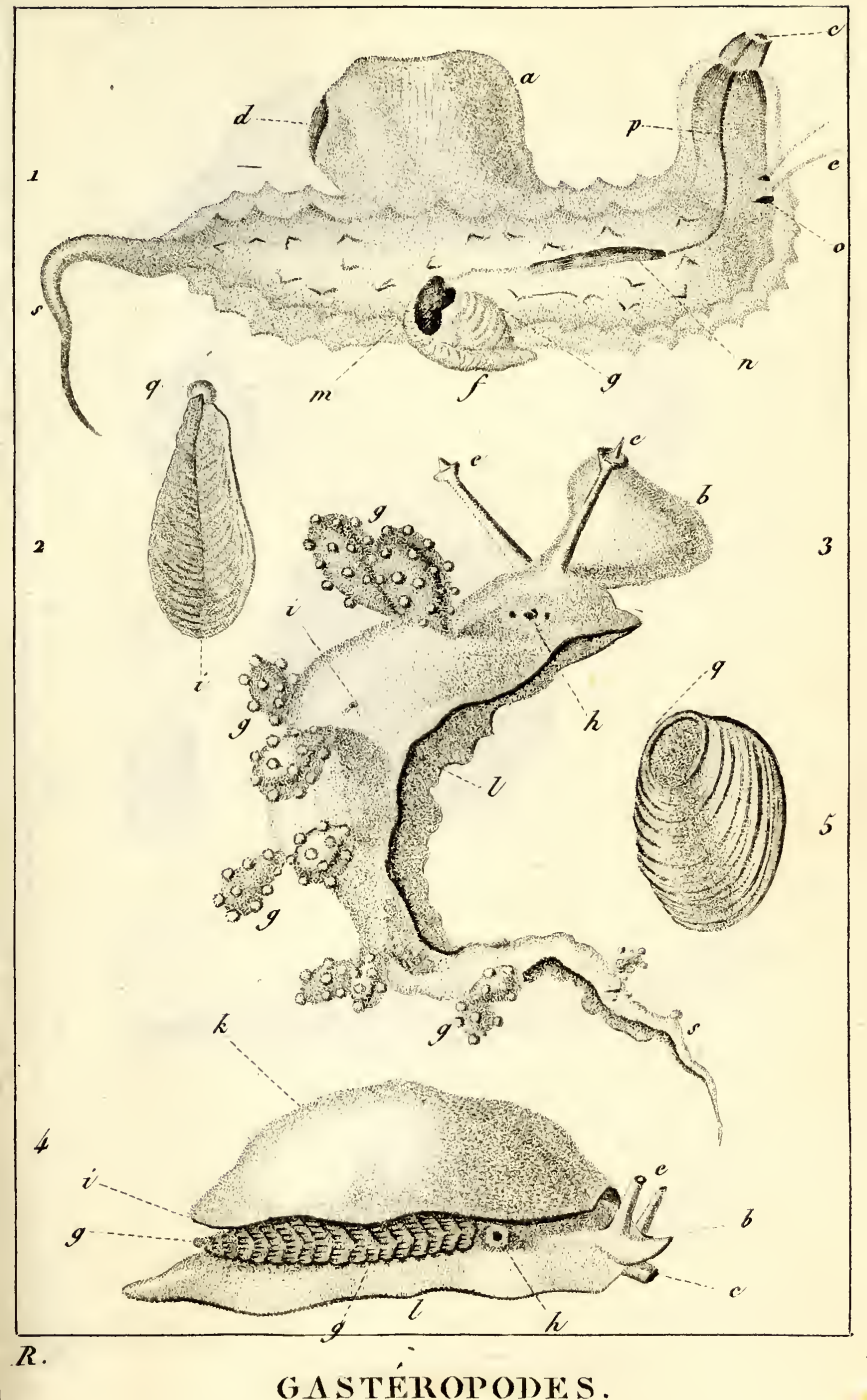


(2) 


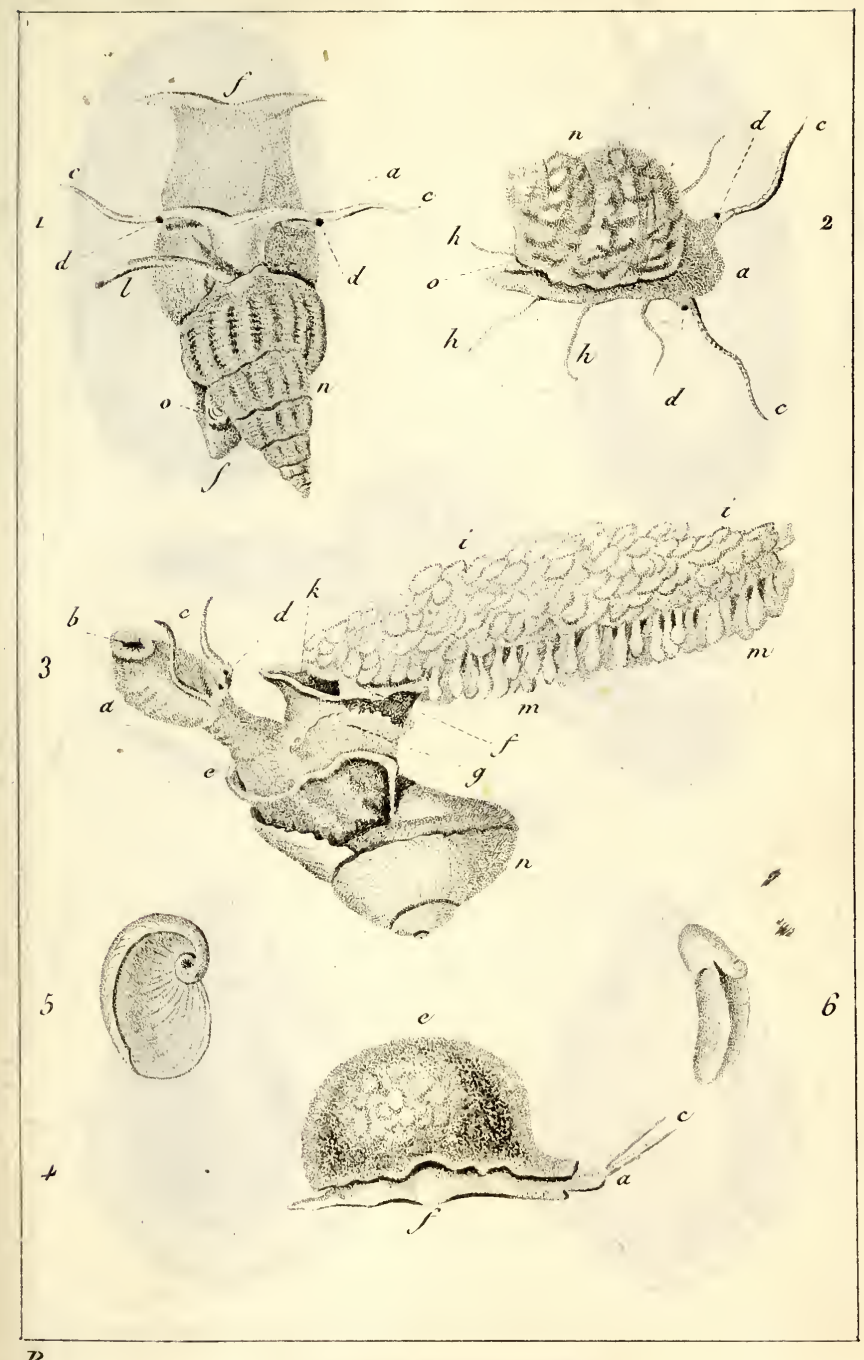

GASTEROPOI)ES . 

Pl.VI.

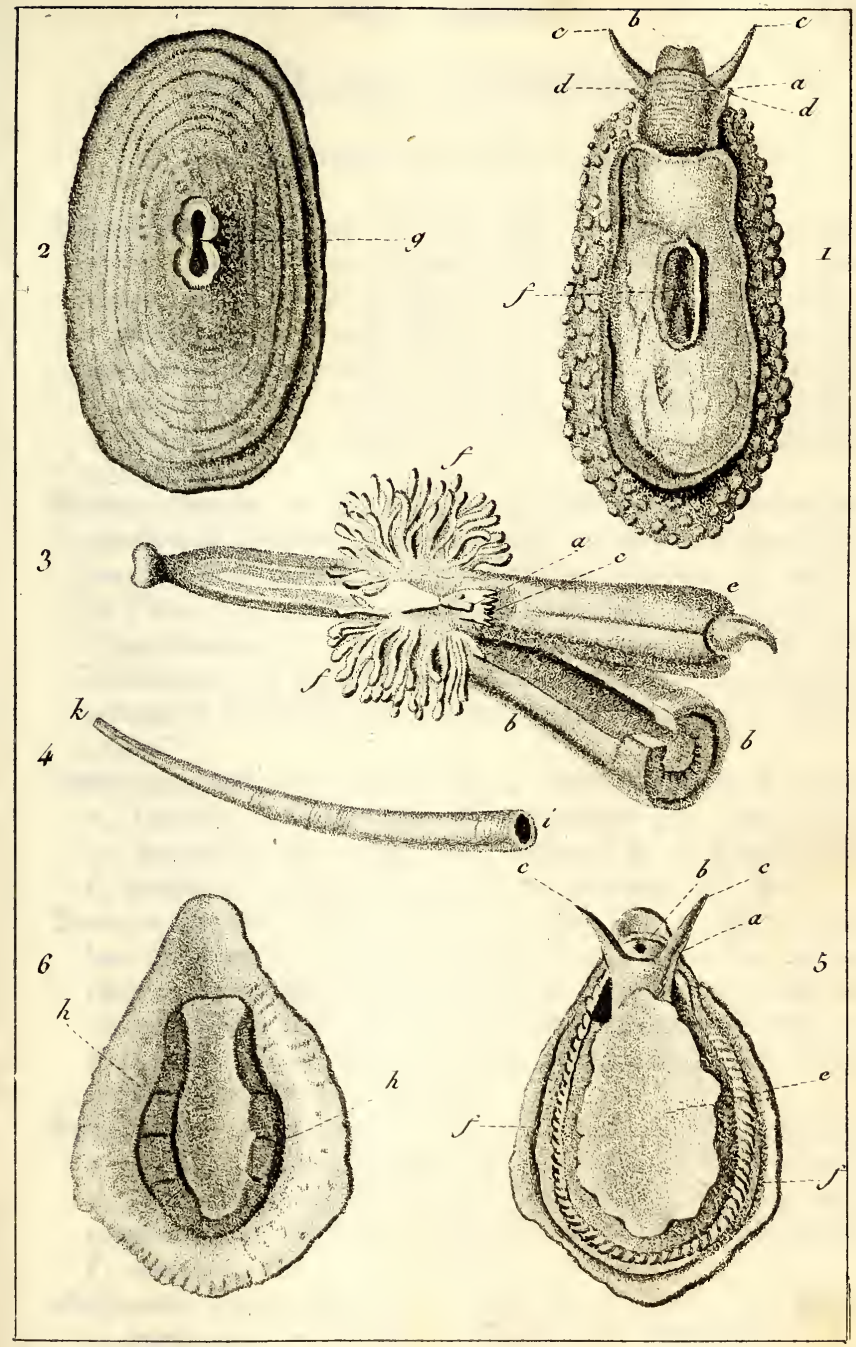

R.

G ASTÉROPODES. 



\section{III' CLASSE. GASTÉROPODES.}

\section{Planches III, IV, V et VI.}

Nugléobranches. - Pl. III, fig. I. Carinaire; $a, n a-$ geoire; $c$, bouche à l'extrémité d'une trompe; $d$, ventouse, ou dernier vestige du pied dans la nageoire; $e$, tentacules; $f$, coquille située à la face dorsale, qui est toujours l'inférieure; $g$, peigne branchial; $m$, ovaire; $n$, masse du foie et de l'estomac; $o$, yeux; $p$, resophage; $s$, queue. Fig. 2. Coquille de cette dernière; $q$, sommet; $r$, carène.

Nudibranches. - Pl. III, fig. 3. Mélibée; $b$, voile qui entoure la bouche; $e$, teutacules; $g$, massues branchiales; $h$, orifices des organes de la génération; $i$, orifice de l'anus; $l$, pied; $s$, extrémité caudale. Pl. IV, fig. 4. Placobranche; $c$, tentacules supérieurs; $d$, tentacules inférieurs, ou appendices labiaux; $b$, lobes du manteau servant à la natation, et rabattus sur les côtés; $f$, branchies.

Inférobranches. - Pl. III, fig. l. Pleurobranche; $b$, voile; $c$, bouche à l'extrémité d'une trompe; $e$, tentacules; $g$, branchie; $h$, orifice des organes de la génération; $i$, position de l'anus à droite; $k$, manteau; $l$, pied.

Tectibranches. - Pl. IV, fig. I. Aplysie; $a$, pied; $b$, lobes du manteau relevés sur le dos; $c$, tentacules postérieurs; $d$, tentacules antérieurs ou labiaux; $e$, canal extérienr allant de l'orifice des œufs à l'organe excitateur, à la base du tentacule droit antérieur. Fig. 2 et 3 . Coquille intérieure; $l$, sommet.

Punmonís rnoperculés. Fl. IV, fig. 5. Parmacelle; $a$, pied; $c$, tentacules supérieurs oculés à leur sommet; $d$, tentacules inférieurs; $g$, bouclier; $h$, orifice de la respiration et de l'anus. Fig. 6 et 7. Coquille intérieure; $l$, sommet.

Punmonés operculés. Pl. IV, fig. 8. Hélicine; $a$, pied; $c$, tentacules ; $y$, yeux ; $i$, coquille; $k$, opercule.

Pectinibrancers. Pl. v, fig. 1. Nasse; $a$, tête; $c$, tentacules; $d$, yeux ; $f$, pied; $l$, siphon; $n$, coquille; $o$, opercule. Fig. 2. Monodonte; $a$, tête; $c$, tentacules; $d$, yeux; 
$h$, expansions tentaculaires du manteau; $n$, coquille; $o$, opercule. Fig. 3. Janthine; $a$, tête; $b$, bouche; $c$, tentacules; $d$, yeux; $e$, bord du manteau à l'entrée de la cavité branchiale; $f$, pied, la partie postérieure, qui est plane; $g$, expansion latérale du mantcau servant à la natation; $k$, pied, la partie antérieure, qui forme une sorte de poche; $i$, grappe de vésicules aériennes servant à la suspension du mollusque à la surface de l'eau; $m$, œufs suspendus sous la grappe vésiculaire; $n$, coquille. Fig. 4. Sigaret; $a$, tête; $c$, tentacule; $e$, manteau; $f$, pied. Fig. 5 à 6 . Coquille.

Scutibranches. - Pl. VI, fig. I. Animal de la Fissurelle détaché de sa coquille; $a$, tête; $b$, bouche à l'extrémité de la trompe; $c$, tentacules; $d$, yeux; $f$, ouverture dorsale de la cavité branchiale. Fig. 2. Coquille de la Fissurelle; $g$, sommet percé d'une ouverture correspondant, sur l'animal, à celle de la cavité branchiale:

Cirriobranches. - Pl. VI, fig. 3. Dentale; $a$, tête; $b$, manteau; $c$, appendices tentaculaires; $e$, pied; $f$, branchies. Fig. i. Coquille du Dentale; $i$, ouverture antérieure : $\bar{k}$, ouverture postérieure.

Cyclobranches. - Pl. VI, fig. 5. Patelle avec son animal vu en dessous; $a$, tête; $b$, bouche; $c$, tentacules; $e$, pied; $f$, branchies. Fig. 6. Patelle privée de son animal, et vue en dessous; $h$, impressions musculaires interrompues en avant.

\section{CLASSE. ACÉPHALES.}

\section{Planches VII et VIII.}

Brachiopodes. - Pl. VIII, fig. I. Térébratule avec ses deux valves fermées; $h$, pédoncule fibreux, propre à fixer l'animal avec sa coquille aux corps sous-marins. Fig. 2. La même montrant l'intérieur de sa peiite valve; $n$, la charpente qui la distingue. Fig. 3. La même présentant la grande valve; $o$, ouverture pour le passage du pédoncule.

Ronistes. - Pl. VII, fig. 5. Sphérulite privée de sa valvé supérieure; $s$, valve inférieure; $t$, birostre; $u$, appareil accessoire. 
श.णn.

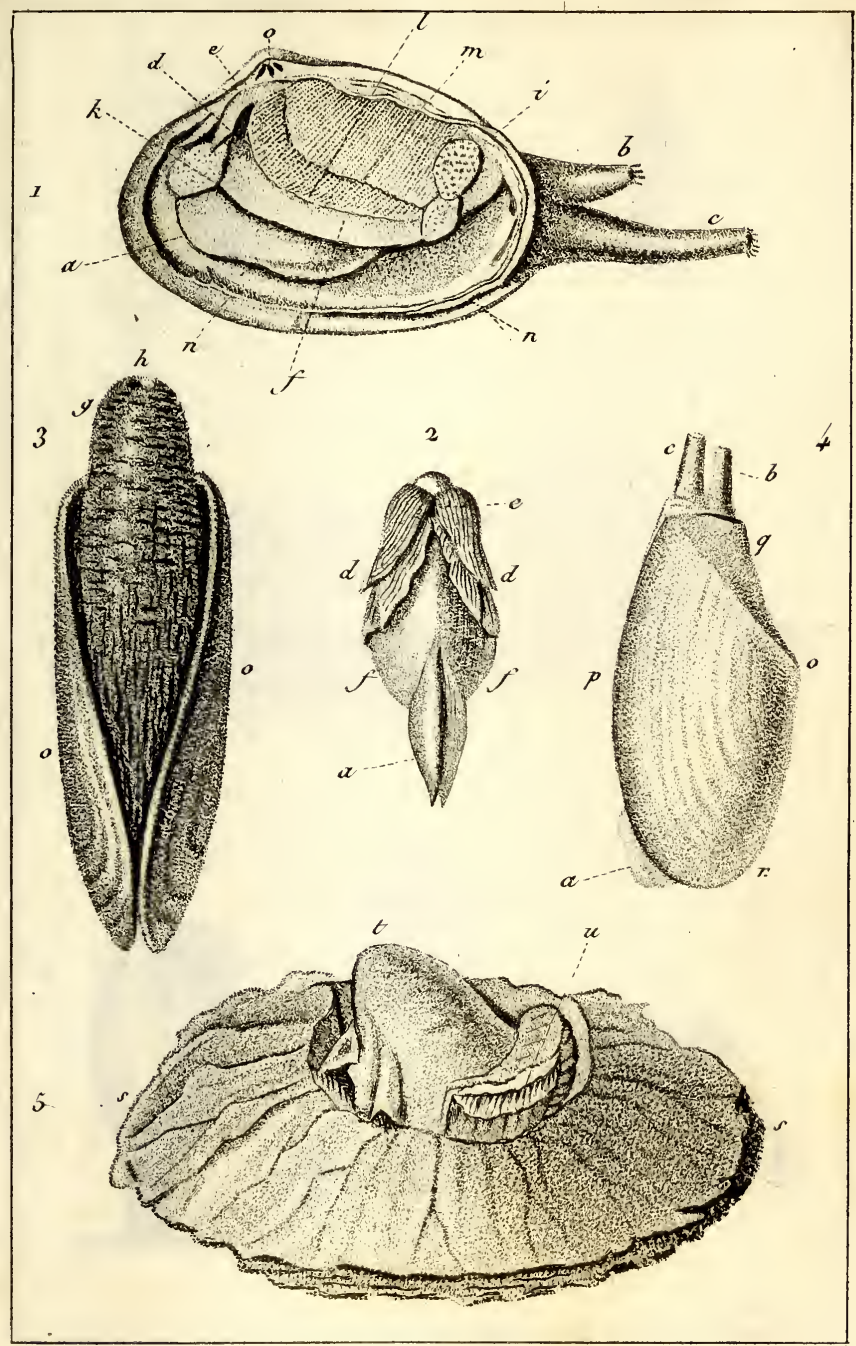

$R$.

ACEPH ILES. 



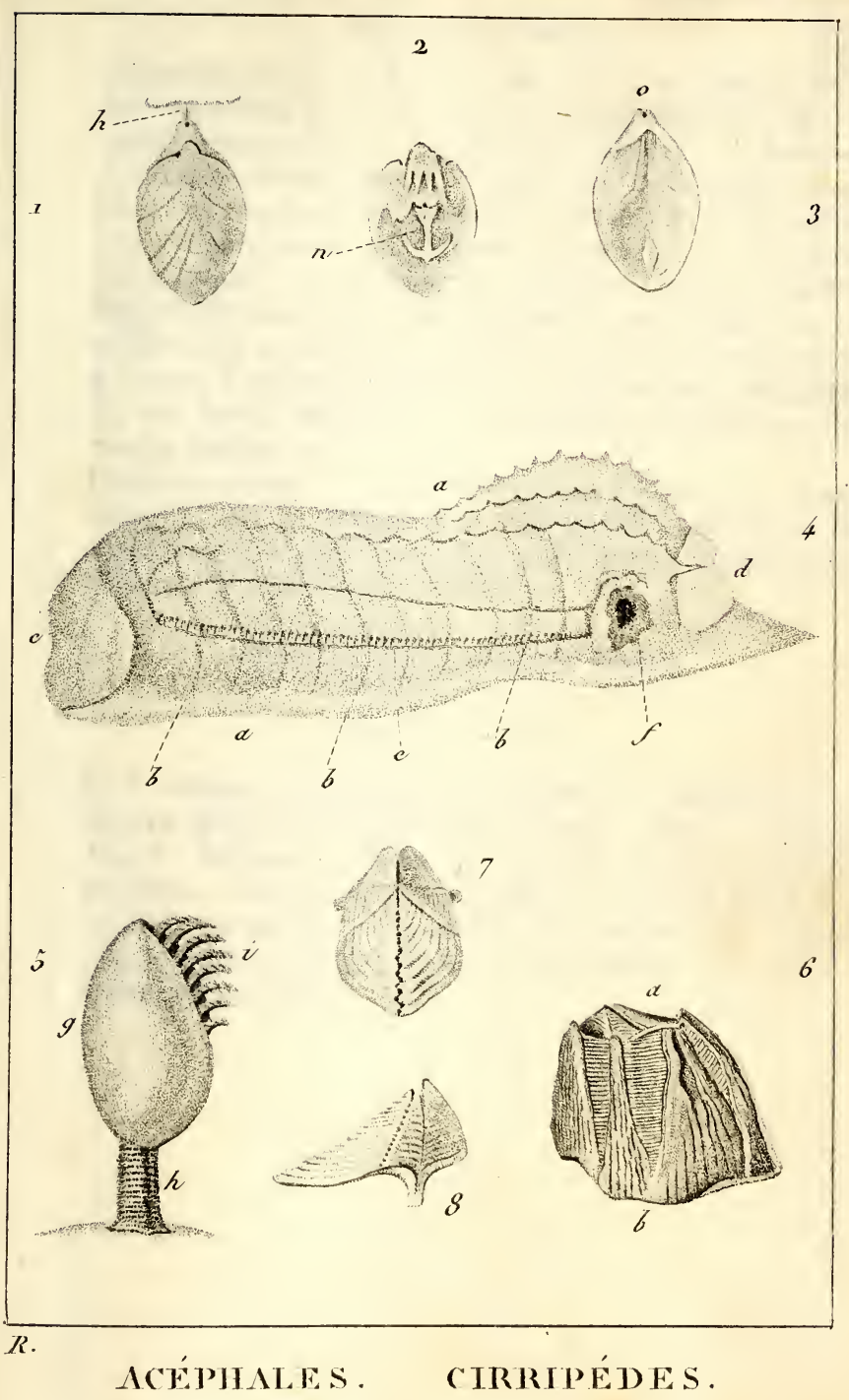



Lamelitbra Nches. - Pl. VII, fig. I et 2. $a$, pied; $b$, tube correspondant à l'anus; $c$, tube communiquant avec la cavité branchiale; $d$, appendices labiaux; $e$, bouche; $f$, masse abdominale; $i$, impression musculaire postérieure; $k$, impression musculaire antérieure; $l$, brauchie, la lame inférieure; $m$, la lame supérieure; $n$, les bords du manteau; $o$, sommet $\epsilon$ charnière de la coquille. Fig. 3. Glycimère; $g$, tube unique; $h$, son orifice; $o$, coquille. Fig. 4. Anatine; $a$, pied; $b$, tube correspondant à l'anus: $c$, tube des branchies; $o$, sommet de la coquille; $p$, son bord inférieur; $q$, sa partie postérieure; $r$, sa partie antérieure.

Hétérobranches. -- Pl. VIII, fig. 4. Biphore; $a$, manteau; $b, b, b$, muscles; $c$, ouverture postérieure du manteau; $d$, ouverture aũtérieure; $e$, écharpe branchiale; $f$, masse des viscères, ou nucléus.

\section{$\mathrm{V}^{\mathrm{e}}$ CLASSE. CIRRIPÈDES.}

\section{Planche VIII.}

Fig. 5. Alèpe; $g$, manteau de l'animal; $h$, pédoncule au moyen duquel il se fixe aux corps; $i$, bras articulés. Fig. 6. Balane; $a$, l'ouverture présentant l'opercule en position, l'animal étant contracté; $b$, la base de la coquille, au moyen de laquelle elle se fixe aux corps. Fig. 7. Opercule de Balane vu en dessus. Fig. 8. Le mème vu par côté. 


\section{TABLE ALPHABÉTIQUE.}

A.

Acarne.

Acarde.

Acardes.

Acaste.

Acave.

Acéphales.

Acéphales non testacés.

Acéphales testacés.

Acère.

Acères.

Acérés.

Achatine.

Acheloïte.

Acochlides.

Actéon.

Adelosine.

Adémacés.

Aeolis.

Aganide.

Agathine.

Agathistègues.

Ailées.

Alasmodonte.

Alène.

Alèpe.

Âlvéoline.

Alvéolite.

Amalté.

Amblémide.

Ammimone.

Ammonées.

Ammonelliptite.

Ammonie.

Ammonite.
Ammonocérate.

Amphibulime.

Page ${ }_{9}^{6}$ Amphidesme.

I 40 Amphitégine.

270 Amplexus.

372 Ampullaire.

158, I6o Anatife.

256 Anatifes.

Anatine.

257 Anaulace.

I 46 Ancillaire.

ibid. Ancille.

ibid. Ancyle.

I 58 Andromède.

${ }_{96}$ Anguinaire.

84 Angulite.

144, I46, 375 Anodonte.

I Io Anomaline.

336 Anomie.

I28 Anostome. I58, 16r, 162

92 Anténore. 108

I66 Antliobrachiophores. 83

rog Aplide. 355

208 Aplysie. $\quad 143$

294 Aplysiens. 142

224 Aporobranches. $\quad 13$

364 Arcacés. $28 \%$

I I Arche.

ibid.

97, I I Archidie.

94 Archonte.

294 Argonaute.

96 Arion.

93 Arminie.

94. Arrosuir.

92 Arthémis.

94 Articuline.
III

114

84 , I2 I

I53, 154

136

337

II0
3 I 6 
TABLE ALPHABÉTIQUE.

Ascidie.

Ascidiens.

Asiphonoides.

Assiline.

Astacole.

Astarté.

Atlante.

Atlantides.

Atlas.

Auriculacés.

Auricule.

Auricules.

Aurifère.

Auriformes.

Avicule.

Aviculés.

Azémus.

B.

Baculite.

Balane.

Balanes.

Balanides.

Batolite.

Bécasse.

Bélemnite.

Bellérophe.

Berthelle

Biapholius.

Bifaribranches.

Bifores.

Bigénérine.

Biloculine.

Bipapillaire.

Biphore.

Birostrite.

Bisiphite.

Borélie.

Boscie.

Botrylle.

Brachiopodes.

Branchifères. $\begin{aligned} \text { Page } 35 \mathrm{r} & \text { Briarée. } \\ 350 & \text { Bronte. }\end{aligned}$

97 Bucarde.

IOg Buccin.

ror Bulime.

314 Bulimine.

I23 Bulle.

ibid. Bullée.

374 Bulléens.

I 72 Bursatelle.

I73 Byssomie.

Page r26

$2 \times 7$

304

209

158

104

147,148

ibid., ibid.

I42, 146

145

334

172

365

240 Cabochon.

286 Cabochons.

245

241

285 Cadran. 202

370 Calcarine. 105

Calcéole. $26 x$

Callirhoé. $\quad 96$

93 Calmar. $\quad 89$

37 i Calmaret. 87

367 Calpurne. 232

ibid. Calyptraciens. $239,241,246$

272 Calyptrée. 24 I

217 Camacés. 297

96 Came. ibid.

85 Canalifères. 206, 208

x39 Cancellaire. 215

335 Cancride. 108

336 Canthrope. $\quad 9^{2}$

337 Caprine, 299

roI Capse. 305

ro9 Caracolle. 158, ז62

35.2 Cardite. 295

357 Carichie. $\quad 172$

270 Carinaire. I2 I

y2 Casque. 2 I4

I I Cassidaire. ibid.

366 Cassiduline. I0?

354 Castalie. 294

257 Catille. 285

245 Cavoline. I 4 , 28 
Cellulée.

Céphalophores.

Céphalopodes.

Page 107 Clisiphonte.

83 Clithon.

Céphalop. non testacés. 84 Clotho.

Céphalop. test. monoth. ibid. Cochlicelle.

Céphalop. test. polyth. 9o Cochlicope.

Cératite.

Cératoide.

Cérite.

Cérites.

Cervicobranches.

Cétocine.

Cétopire.

Chélonobie.

94. Cochlitome.

$9^{3}$ Cochlodine.

207 Cochlodonte.

Chicoracés.

206 Cochlogèue.

239 Cochlohydre.

96 Cochlostyle.

3 yo Colimacés.

ibid. Colombelle.

Page 108

$19^{3}, 194$

349

$32 \mathrm{r}$ I66

ibill.

I 65

I69

ibid.

167

I59

165

×56

2I 7 Columellaires.

Chismobranches. 18r, 239, Conchacés.

219 251.

Concholépas.

Chismob. cricostomes. I 73 Cône.

Chrysaore.

Chrysole.

Chthamale.

Cibicide.

Cidarole.

Cimber.

Cinèras.

Cirrhus.

Cirrhipèdes.

Cirripèdes.

96 Cônes.

ro8 Conie.

37 r Conovule.

ro6 Coralliophage.

208,234

302

212

223

ilid.

370

I 72

320

I04 Corbeille. 3 I 7

r9 Corbicule. 313

365 Corbule. 323

202 Coriocelle. 236

362 Corne d'Ammon vivante. 123

ibid. Coronule. 3 70

Cirripèdes pédonculés. 363 Cortale. 105

Cirripèdes sessiles. $\quad 367$ Cranchie.

Cirrhobranches. $\quad 248$ Cranie.

Cirrhopodes.

362 Cranies.

88

Cithérée.

Clausilie.

Clausulie.

Clavagelle.

Clavatule.

Clavuline.

Cléodore.

315, 3ı 6 Crassatelle.

262

ibid.

314

I58, r 70 Crassine. ibid.

I I I C'rénatule. 383

338 Crépidule. 243

22 I Créséis. 115

ro4 Creusie. $\quad 369$

Ix5 Cristellaire. Iо8

Clio.

Cliodite.

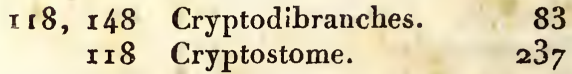

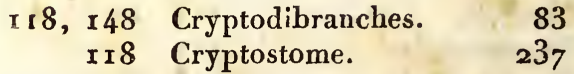

Clios.

xi7 Cuviérie. 
AI,PHABÉTIQUE.

Cyclade.

Page 3 I2 Élédone.

Cyclobranches. 124, 131, 25 r Ellipsolite.

Page 87

Cyclostome.

Cylindre.

Cymbicochlides.

Cymbulie.

Cypricarde.

Cyprine.

Cyrène.

Cyrtodère.

I80 Flphide.

94

I07

223 Filysie.

84 Émarginule.

I 3 Énallostègues.

256, 320 Encælie.

3 ro Enfermés.

313 Enroulés.

332 Entérostés.

Entomostègues.

D.

Dauphinule.

Décacères.

Décapodes.

Dendritine.

Dentale.

Dentales.

Dentaline.

Dermatobranche.

Entonnoir.

Éolide.

201 Éomphale.

87,90
Io7 Érycine.

I 46, 375

246

IOI

374

336

225

84,87

I IO

203

127,128

202

201

312

297

249 Eucélie.

355

I 53

99
373 Euribie. 1 I7

Diadême.

Dianchore.

Diazome.

Dicères.

Dicérate.

Diderme.

Dimorphine.

Dimyaires.

Diphylidie.

Dipsas.

Discine.

Discorbe.

Distome.

Dolabelle.

Donace.

Doridie.

Doris.

370

279 Fabulaire. II I

128, 142 Fasciolaire.

220

298 Fasciolite.

I 1 I

I 80

355,356

102

287

I 36

293

263

105

353

Férussine.

Firole.

120

Firolides.

Firoloïdes.

ibid.

120

Fissurelle.

Fistulaire.

247

348

Florilie.

108

Fodie.

$143,1 / 4$

306

Foraminifères.

Fripière.

146

I3 $1, \mathbf{3} 2$

Frondiculaire.

Fuseau.

352

97

202

100

220

E.

Éburne.

Égéone.

Égérie.

209 Gradus.

xo9 Galathée.

3.3 Gastéropodes.

G. 
Gastéroptère.

Gastrochène.

Gastroplace.

Gemmuline.

Géocochlides.

Géopone.

Gervilie.

Gervisia.

Glanduline.

Glanque.

Glauques.

Globigérine.

Globite.

Globuline.

Glycimère.

Goniatite.

Gratelupie.

Grenaille.

Gryphée.

Guttuline.

Gymnodermes.

Gymnolèpe.

Gymnosomates.

Gyroïdine.

H.

Haliotide.

Hamite.

Hammonie.

Harpace.

Harpe.

Helcion.

Hélénide.

Hélicarion.

Hélice.

Hélicelle.

Hélicigone.

Hélicine.

Hélicines.

Helicinides.

Hélicite.

Hélicodonte.
Page 148 Hélicogène.

342 Hélicolimace.

Page 160

I íco Hélicophante.

102 Hélicostègue.

I56 Hélicostyle.

ro7 Hémicarde.

284 Hermès.

139 Héfione.

99 Hétérobranches.

125 Hétéropodes.

ibid. Hétérostégine.

105 Hiatelle.

94 Hibolite.

ro3 Hinnite.

332 Hippocrène.

94 Hipponice.

3 o 7 Hippope.

I69 Hippurite.

276 Hiptère.

Io3 Homolocératite.

263 Hortole.

367 Houlette.

II 7 Huitre.

105 Hyale.

Hyales.

Hyrie.

240

94

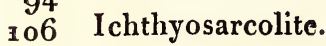

274 Ilote.

2 I0 Inférobranches. $135, \mathrm{r} 36$

$25 \mathrm{I}$ Inocerame. 284

II I Iridine. 303

${ }_{25} 5$ Isocarde. 300

I58, 160 J.

162 Jambonneau.

ibid. Janthine.

179 Jodamie.

I78 Jouannétie.

ibid.

Io9

16. Kraken.
I.

96

I I I

157

159

ro3

164

Зоร

223

108

350

II)

III

335

$9^{6}$

278

$22 x$

244

302

272

I2I

ibid.

279

275

II 4

II 2

294

292

r $9^{6}$

270

345

K.

86 
A乙PHABÉTIQUK.

$\begin{array}{rrlr}\text { I. } & & \text { Lithotrie. } & \text { Page } 367 \\ & & \text { Litiope. } & \text { r97 } \\ & \text { Page } 200 & \text { Littorine. } & \text { I85 } \\ & \text { r39 } & \text { Lituite. } & 93,108 \\ & 253 & \text { Lobaire. } & 146 \\ \text { ches. } & 272 & \text { Loripède. } & 308 \\ & \text { 108 } & \text { Lucine. } & \text { ibid. } \\ & \text { I26 } & \text { Lutraire. } & 327\end{array}$

Lacuna.

Lamellaire.

Lamellés.

Lamellibranches.

Lampadie.

Laniogère.

Laplysie.

Laplysiens.

Lastène.

Léachie.

Lenticuline.

Lenticulite.

Lépadiens.

Licophre.

Licorne.

Lièvre marin.

Ligule.

Limace.

Limacelle.

Limaces.

Limaciens.

Limacine.

Limacines.

Liwacinés.

Limaçons.

Lime.

Limnacés.

Limnée.

143,144

142

293 Macome.

MP.

87 Macrodite.

Io6 Macrostome.

Iog Mactre.

363 Magas.

og Magile.

21 I Maillot.

I 43 Malléacés.

327 Margaritacés.

153, 154 Marginelle.

r53 Marginule.

I5o Marteau.

318 108

240

3 r 1

258

I8s

I58, $x 6_{9}$

28 I

ibid.

233

I0o

283

ibid. Mélampe.

173

Ix3 Mélanie.

183

123 Mélanopside. 204, 205

15o, э56 Méléagre.

I56 Mélibée.

200

280 Mélonie.

129

$10 \$$

175 Milioles. 109

I76 Miliolite. 111

I75 Minaret. 226

Limnéens.

Limnocochlides à collier.

Limnocochlides sans collier.

Linguelle.

Lingule.

Lingules.

Linguline.

Lintlurie.

Lithodome.

Litholèpe.

Mitre.

I72 Modiole.

ibid.

Monodonte.

200

201

175 Monomyaires.

273

I36 Monophore. 36r

257 Monopleurobranches. $\quad \mathbf{4 2}$

ibid. Moule.

roo Mucronine. roo

ro8 Mulette. 294

29 I Mye. 326

367 Mytilacés. 290 
N.

Nasse.

Natice.

Nautilacés.

Nautile.

Navette.

Navicelle.

Nectopodes.

Nématopodes.

Nériné.

Nérite.

Néritine.

Nodosaire.

Nonionine.

Notarche.

Notrême.

Nucléobranches.

Nucule.

Nudibranches.

Nudilimaces.

Nummulie.

Nummulines.

Nummulite.

\section{O.}

Océanie.

Ochthosie.

Octocères.

Octomère.

Octopodes.

Ocythoé.

Olive.

Olygyre.

Ombrelle.

Ombrelles.

Onchide.

Onchidie.

Onchidore.

Onguline.

Onychie.

Ony choteuthes.

Operculine. Page 106

pape 3 Ophiopormophite.

Orbicule.

r9o, r9r Orbiculine.

9I Orbulite.

92 Oréade.

232 Oréas.

19 I Orizaire.

120 Ormiers.

362 Orthocératite. $\quad 93,272$

208 Orthocère. 98 , roo

193, 194 Orthocérine. 100

I 94 Oscabrelle. 254, 255

98,99 Oscabrion. 254

I 8 Oscabrions. 253

143,145 Ostéodesme. 325

244 Ostracés. 273, 276, 28 r.

I 19 Ostracodermes. 363

288 Otidés. 240

r24 Otion. 365

150 Ovule. 232

ro9 Oxigones. $28 \mathrm{r}$.

ibid. Oxinoé. 375

ibid. Oxitrème. ibid.

Ozoéma. 87

P.

${ }_{369}^{92}$ Paclite. $\quad 96$

84 Padole. 238

373 Paludine. $\quad 182$, I83

84 Pandore. 323

ibid. Panopée. 333

228 Paracéphalophores. II9

I79 Parmacelles. I54

I40 Parmophore. 246

I37 Parthénope. I48

I5 I Partule. I7I

15I, 152 Patelle. I21, 25 I

133 Patelles. $25 \mathrm{r}$

32 I Patelloides, $\quad \mathbf{3} 7,245$

88 Patrocle. 108

ibid. Pavonie. roi 
ALPHABÉTEUE .

Pectinibranches.

P. 78, I8r

Planaxe.

Pectinides.

Peigne.

Pélaguse.

Pélore.

Péloronte.

Pénérople.

Pentadine.

Pentalasmis.

Pentalèpe.

Pentastère.

276 Planorbe.

2.78 Planorbite.

94 Planorbuline.

Page 205

175

107 Planulaire.

94

106

IOI

193, 193 Planuline.

I 06

I07 Planulite.

286 Plectrophore.

94

366 Pleurobranche.

155

ibid. Pleurobranchie. 38

258 Pleurobranches. $\quad$ 3 37

Perdrix.

Péribole

Périplome.

Péristellées.

Péronie.

Perne.

Pétoncle.

Pétricole.

Pharame.

Phasianelle.

Pholadaires.

Pholade.

Pholades.

Phonème.

Phos.

Phyllidie.

213, 21/4 Pleurobranchidie.

r38

23o Pleurocère.

325 Pleurotomaire.

374

$9^{5}$ Pleurotome. $22 \mathrm{I}$

I5 I Plicatule.

278

$2 \mathrm{~S}_{2}$ Pneumoderme. $\quad 118$

288 Pneumopomes. $\quad$ I78

320 Podopside. 277

ro8 Polinice, I9o, r91

198 Polixène.

326 Pollicipède.

106

343 Pollonte.

366

110

I08 Polycère.

218 Polycline. 354

I36 Polycycle. ibid.

Phyllidiens. 135, 136, 25 I Polydonte. I58, 162

Phyllidies.

Phylliroé.

Phyllobranches.

I36 Polylèpe.

36 o Polymorphine.

366

ז25 Polyphême. 158,166

Phylomique.

Physe.

Piétin.

153 Polyplaxiphores. 107, 1 19,

$17725 \mathrm{I}, 2 \tilde{5} 3$

Piléiforme.

I 7 Porcelaine.

Piléole.

Placentule.

Placobranche.

Placobranches.

Placune.

Plagiostome.

24I, 245 Porcellane.

230

233

$19^{3}, \times 9^{5}$ Porodrague.

I07 Posidonie.

96

134 Potamide.

ibid. Potamophile.

$28 \mathrm{I}$

274 Poulpe.

280 Pourpre. 
Pourpres.

Procéphales.

Producte.

Proto.

Psammobie.

Psammocole.

Psammotée.

Psyché.

Ptérocère.

Ptéropodes.

Ptérosome.

Ptérosomes.

Ptérotrachée.

Ptérotrachées.

Pulmobranches.

Pulmonelle.

Pulmonés inoperculés

Pulmonés operculés.

Pulvinite.

Purpurifères.

Pyloridés.

Pyraze.

Pyrène.

Pyrgo.

Pyrgome.

Pyrgopole.

Pyrosome.

Pyrule.

Pyruline.

Pyure.

Q.

Quadrifores.

Quinqueloculine.

R.

Radiolite.

Ranelle.

Raphaniste.

Rénuliue.

Rémuline.

Réophage.
Payge 208 Rétifères.

123 Rhabdite.

26 r Rhinocure.

I86 Rhombe.

328, 329 Rhomboïde.

328 Ricinule.

328,329 Rimuline.

I 7 Rimule.

222, 223 Rissoaire.

I12, 123 Robule.

I 25 Robuline.

r24 Rocher.

I20 Rosaline.

II9, 120 Rostellaire.

I49 Rotalie.

355 Rotelle.

r49 Rouleau.

178 Rudistes.

285 Rupellaire.

208 Rupicole.

322,336

208

204,205

373

368

96

$36 r$

2r9

103

353

Sabot.

Sagitelle.

Salpiens.

Sanguinolaire.

Saracenaire.

Saxicave.

Scalaire.

Scalpelle.

Scaphite.

Scarabe.

$104,105,109$

I9o, I $9 \mathrm{~d}$

223

263

320

325

S.

200

120

356

329

108

333

204

366

94

174

108

367 Scortine.

I 10 Scutibranches. $\quad 239$

Scyllée.

Seiche.

270 Seiches.

2 r 8 Semi-phyllidiens.

उ 30

89

84

I 35

272 Sépiaires.

ro7 Sépiole.

84,87

roo Sépiolés.

88

87

98 Sépiotheuthe. 
ALPHABÉTIQUE.

Fage I91 Strophostome. Page 180 227 Struthiolaire.

r28 Strygocéphale.

ro9 Subaplysiens.

105, rog Submytilacés.

236 Subostracés.

iłid. Succinée.

353

187

94 Taret.

355 Tarière.

$14 \mathrm{I}$ Tectibranches.

90 Télescope.

204 Telline.

ro6 Tellinide.

33o Ténagode.

33 r Tentaculés.

33o Térébratule.

336 Térébratules.

322, 336 Térédine.

329 Térédinites.

I49 Tergipe.

323 Tergipède.

Io3 Testacelle.

270 Tétracères.

I 8 Textulaire.

1 i Thalamule.

I/4. Thécidée.

258 Thécosomes.

ros Théméone.

rog Théthys.

9I Thracie.

ibid. Timorienne.

277 Tinopore.

ro7 Tirannite.

98 Tomogère.

238 Tonne.

iüid. Toupie.

222 Tonrbillons.

ibid. Toxérite.

$29^{3}$ Trachélipodes, 1 19, $156^{\circ}$,

$25 \mathrm{r} \quad \mathrm{r} ; 8,28 \mathrm{r}, 239$

2 18

258

$\times 35, \times 37$

293

275

×58, 159

T.

346

227

142

203

$3 \circ 8$

ibid.

$\mathbf{1} 87$

142

258

ibid.

350

336

r28

ibid.

I55

125

102

$9^{6}$

26 r

I 12

107

129

324

359

105

$\times 58, \mathbf{1 6} \mathrm{r}$

$2 \times 3,2$ I 4

199,203

I 62

Strophite.

Strophonême. 
TABLE ALPHABÉTIQUE.

Trémésie.

Tricle.

Tridacne.

Trigonie.

Triloculine.

Triphore.

Tripter.

Triptère.

Triquètre.

Tristome.

Triton.

Tritonie.

Tritonies.

Page 244 Uvigérine.

I 14

$30 \mathrm{I}$

289 Vaginelle.

I 10 Vaginule.

I 16

208 Vaginuline.

I52

Valvée.

Page 104

V.

I 6 , 2r7 Valvuline.

318 Vélate.

208 Vélutine.

100

I 89

104

95

238

218,36 q Vénéricarde.

295

I 3 V Vénérupe. 3 r9

128, i ว I Vénus. $3 \times 5$

Tritoniens. 13 r

Trochoïdes.

Trochuline.

Troncatuline.

Tubicinelle.

Tubicolés.

Turbicines,

Turbinelle.

Turbinés.

Turbinuline.

Turrilite.

Turritelle.

Tylodine.

Typhis.

Vermet.

Vermiculaire.

186

ibid.

r9r Véronicelle. $\quad 152$

105 Verrue.

Io6 Vertébraline.

369

370 Vertigo.

370 Vertigo. 170

336 Virguline. 103

r79 Vis. 225

219 Vitrine. 157

182 Volute. ibid.

I05 Volutes. 234

95 Volvaire. 233

185 Vorticiale. 107

374 Vulselle. $2 \mathrm{S2}_{2}$

218 Vulvuline. 102

U.

Ultime.

Unabranches.

Uniodié.

Urobranches.

Y.

232 Yet.

235

137

294

120,131 Westernie.

Y.

W.

139

FIN DE LA TABLE.

1)E L'IMPRIMERIE DE GRAPELE'

rue de Vaugirard, $\varkappa^{\circ} 9$. 


50,211150 


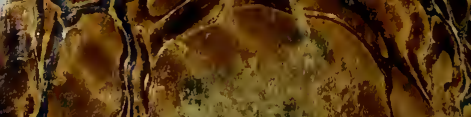

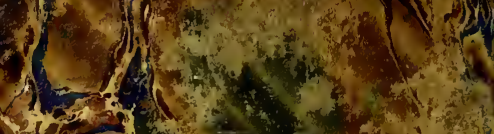

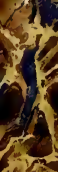

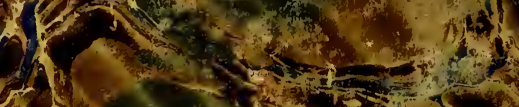

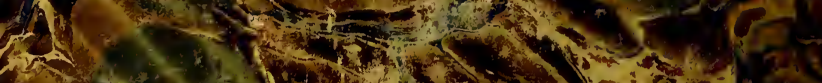

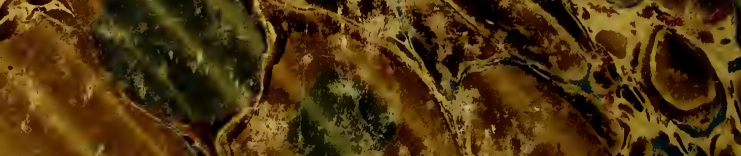

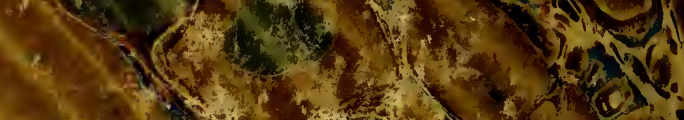

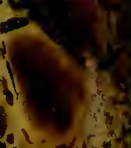

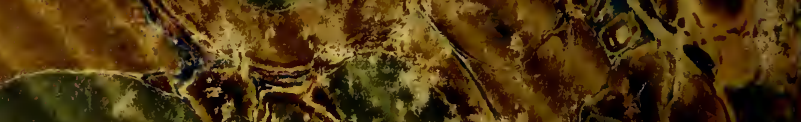

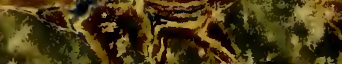

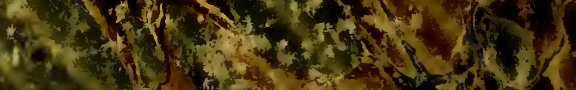

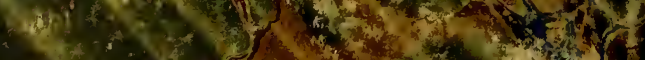

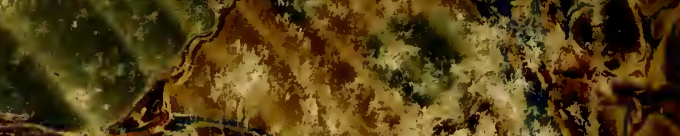

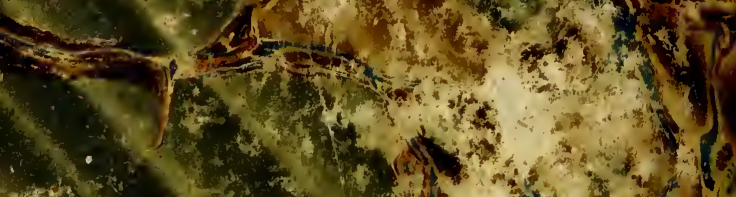

(5)

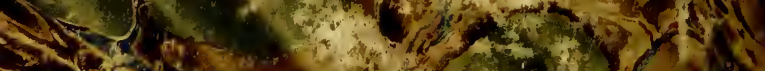

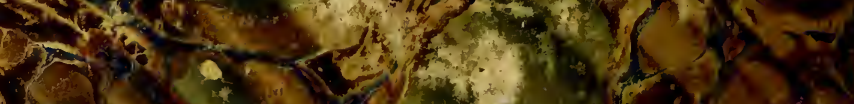

(1)

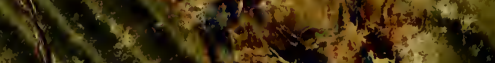

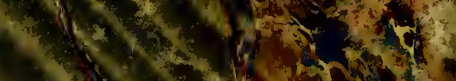

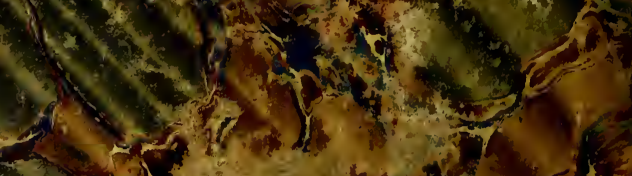

and

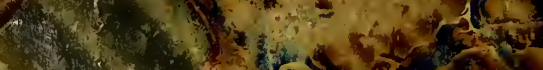

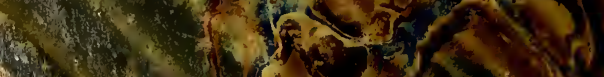

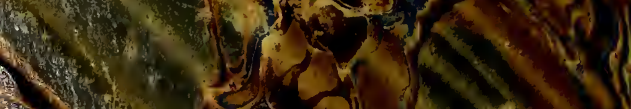

